U.S. GEOLOGICAL SURVEY APPLIED RESEARCH STUDIES

OF THE CHEYENNE RIVER SYSTEM, SOUTH DAKOTA:

DESCRIPTION AND COLLATION OF DATA,

WATER YEARS $1985-86$

Edited by Kimball E. Goddard

U.S. GEOLOGICAL SURVEY

Open-File Report $88-484$

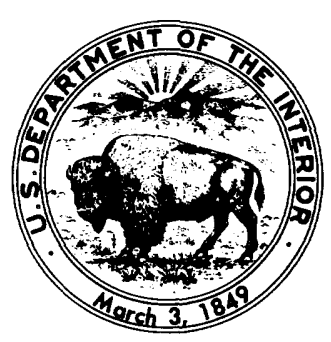

Huron, South Dakota 1988 


\author{
DEPARTMENT OF THE INTERIOR \\ DONALD PAUL HODEL, Secretary \\ U.S. GEOLOGICAL SURVEY \\ Dallas L. Peck, Director
}

For additional information write to:

Subdistrict Chief U.S. Geological Survey Rm. 237, 515 9th St. Rapid City, SD 57701
Copies of this report can be purchased from:

U.S. Geological Survey Books and Open-File Reports Federal Center, Bldg. 810 Box 25425 Denver, CO 80225-0425 
Abstract ............................ . 1

Introduction . . . . . . . . . . . . . . . . . . . . . 1

Problem . . . . . . . . . . . . . . . . . . . . . 2

Purpose and scope ....................... 6

Chapter A. Geochemical characteristics of contaminated sediments

from deposits along whitewood Creek

Physical and chemical characteristics . . . . . . . . . . . . . . 7

Sample collection and handling procedures . . . . . . . . . 7

Analytical procedures and results . . . . . . . . . . . . . . 7

Adsorption/desorption characteristics . . . . . . . . . . . . . . . 9

Sample collection and handling procedures . . . . . . . . . . 9

Analysis of physical and chemical properties . . . . . . . . . . 12

Determination of adsorption/desorption characteristics . . . . . . 16

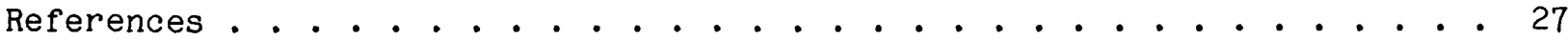

Chapter B. Trace-element geochemistry of sediments collected from the flood plains of Whitewood Creek and the Belle Fourche and Cheyenne Rivers

Sample collection and handling procedures . . . . . . . . . . . 28 Analytical procedures and results . . . . . . . . . . . . . . 28 References . . . . . . . . . . . . . . . . . . . . 28

Chapter C. Trace-element geochemistry of bottom sediment samples collected from Whitewood Creek and the Belle Fourche and Cheyenne Rivers

Sample collection and handling procedures . . . . . . . . . . . . 37 Analytical procedures and results . . . . . . . . . . . . . . . 37

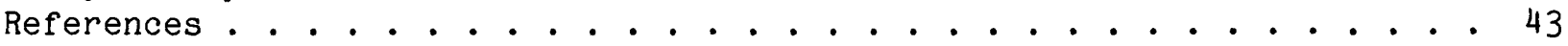

Chapter D. Trace-element geochemistry of sediment cores collected from the Cheyenne River arm of Lake Oahe

Sample collection and handling procedures . . . . . . . . . . . 44 Analytical procedures and results . . . . . . . . . . . . . . . 44

References........................ . 46

Chapter E. Discharge and surface-water quality at selected sites on Whitewood Creek and the Belle Fourche and Cheyenne Rivers

Methods . . . . . . . . . . . . . . . . . . . . 49

Discharge data . . . . . . . . . . . . . . . . . . 49

Water-quality data . . . . . . . . . . . . . . . . 58

References . . . . . . . . . . . . . . . . . . . . 117 
Chapter F. Arsenic and iron versus filter poresize in Whitewood Creek and the Belle Fourche River

Sample collection and handling procedures . . . . . . . . . . . . 118

Analytical techniques and results . . . . . . . . . . . . . . . . 118

References . . . . . . . . . . . . . . . . . . . . . 121

Chapter G. Ground-water geochemistry along Whitewood Creek

Sample collection and handling procedures . . . . . . . . . . . . 122

Analytical techniques and results . . . . . . . . . . . . . . 123

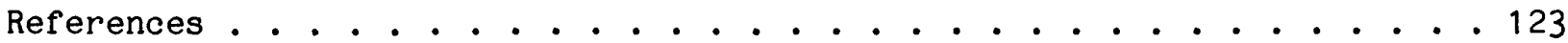

Chapter H. Colloids in seeps and springs along Whitewood Creek

Introduction . . . . . . . . . . . . . . . . . . . . . 125

Sample collection and handling procedures . . . . . . . . . . . . . 125

Analytical procedures and results . . . . . . . . . . . . . . . 126

References . . . . . . . . . . . . . . . . . . . 139

Chapter I. Desorption of arsenic from iron oxyhydroxide

Sample collection and handling procedures . . . . . . . . . . . . 140 Analytical procedures and results . . . . . . . . . . . . . . . . . 141 Desorption experiments and results . . . . . . . . . . . . . . . . 141 References ........................ . . 141

Chapter J. Biological investigations - Surface-water toxics program,

Whitewood Creek and the Belle Fourche and Cheyenne Rivers,

South Dakota

Introduction . . . . . . . . . . . . . . . . . . 148

Work on periphyton....................... . . 148

Arsenic in benthic insects . . . . . . . . . . . . . . . 151

Selected references . . . . . . . . . . . . . . . . 157 
Figure 1. Map showing locations of important data-collection sites within the Whitewood Creek drainage basin . . . . . . . . . 3

2. Map showing locations of the data-collection sites on the Belle Fourche and Cheyenne Rivers . . . . . . . . . . 4

3. Map showing location of sediment cores taken from the Cheyenne River arm, Lake Oahe, during August 1985 . . . . . 45

4. Graphs showing arsenate and iron versus filter poresize:

Whitewood Creek/Belle Fourche River, August 1986 . . . . . 120

5-13. Photomicrographs and $x$-ray spectra for:

5. Particle 01 retained on 100-nm filter, seep sample . . 130

6. Particle 02 retained on 450-nm filter, pool sample . . 131

7. Particle 03 retained on 450-nm filter, pool sample . . 132

8. Particle 04 retained on 100-nm filter, pool sample . . 133

9. Particle 05 retained on 100-nm filter, pool sample . . 134

10. Particle 06 retained on 100-nm filter, pool sample . . 135

11. Particle 07 retained on 100-nm filter, pool sample . . 136

12. Particle 08 retained on 100-nm filter, pool sample . . 137

13. Aggregate retained on 450-nm filter, Whitewood Creek sample . . . . . . . . . 138 
TABLES

Page

Table 1. Names of data-collection sites discussed in text....... . 5

2. Sample number, source, description, and location for sediment

samples collected in April 1986 . . . . . . . . . . . . 7

3. Total analysis of representative tailings and contaminated

sediment samples . . . . . . . . . . . . . . . 8

4. Semi-quantitative D.C. arc spectrographic analysis of specific gravity and magnetic separation splits of representative tailings and contaminated sediment samples . . . . . . . . 10

5. Sequential digestion procedure and arsenic concentrations measured in representative tailings and contaminated sediment samples . . . . . . . . . . . . . . . 12

6. Mineral composition of samples from the Berger site (October 1985), as determined by $x$-ray diffraction analysis . . . . . 13

7. Weight percent of major oxides for samples from the Berger site, as determined by $x$-ray diffraction analysis (October 1985) . . . . . . . . . . . . . . . . . . . . 14

8. Grain-size analysis of samples from the Berger site (October 1985) . . . . . . . . . . . . . . . 15

9. Summary of permeability test results of samples from the
Berger site (October 1985) . . . . . . . . . 16

10. Reaction vessel adsorption characteristics of contaminated sediment samples from the Berger site (October 1985) . . . . 18

11. The effect of solid/liquid ratio on adsorption of contaminated sediment samples from the Berger site (October 1985)

12. Equilibrium time of adsorption of contaminated sediment samples from the Berger site (October 1985) . . . . . . . 19

13. The effect of $\mathrm{pH}$ on the arsenic adsorption/desorption of uncontaminated sediments $(A c-1)$ from the Berger site (October 1985) ..................... 20 20

14. The effect of $\mathrm{pH}$ on the arsenic adsorption/desorption of contaminated sediments (Tc-2) from the Berger site (October 1985) . . . . . . . . . . . . . . . . . 21

15. The effect of sulfate on adsorption of uncontaminated $(A C-1)$ and contaminated $(\mathrm{Tc}-2)$ sediments from the Berger site (October 1985) ..................... . . 22

16. Adsorption/desorption variability of contaminated sediments from the Berger site (October 1985) . . . . . . . . 22

17. The effect of grain size on the adsorptive properties of uncontaminated $(\mathrm{Ac}-1)$ and contaminated sediments $(\mathrm{TC}-2)$ from the Berger site (October 1985) . . . . . . . . . 23

18. Hydraulic characteristics, in columns, of uncontaminated $(\mathrm{Ac}-1)$ and contaminated $(\mathrm{TC}-2)$ sediments from the Berger site (October 1985) . . . . . . . . . . . 24

19. Adsorption/desorption of uncontaminated sediment $(A c-1)$ from the Berger site (October 1985) under near field-flow conditions................ . . 25

20. Adsorption/desorption of contaminated sediment (Tc-2) from the Berger site (October 1985) under near field-flow conditions... . . . . . . . . . . 26 
Table 21. Partial digestion analysis of sediment samples collected

during 1985 and 1986 from the flood plains of Whitewood

Creek and the Belle Fourche River . . . . . . . . . . . .

22. Total digestion analysis of sediment samples collected during

1985 and 1986 from the flood plains of Whitewood Creek and

the Belle Fourche and Cheyenne Rivers . . . . . . . . . 30

23. Total digestion analysis of particle-size splits of sediment samples collected during 1985 and 1986 from the flood plains of Whitewood Creek and the Belle Fourche River . . . . . . .

24. Grain-size distributions of selected sediment samples collected during 1985 and 1986 from the flood plains of Whitewood Creek and the Belle Fourche River . . . . . . 36

25. Site number and name, station number, and location for sites at which bottom sediment material samples were collected during August 1985

26. Total analysis of bottom sediment materials collected from Whitewood Creek and the Belle Fourche and Cheyenne Rivers during August 1985 . . . . . . . . . . . . . . . . . .

27. Total recoverable arsenic and trace metals, and total organic carbon analysis of bottom sediment materials collected from Whitewood Creek and the Belle Fourche and Cheyenne Rivers during August 1985 . . . . . . . . . . . . . . .

28. Particle-size analysis of bottom sediment materials collected from Whitewood Creek and the Belle Fourche and Cheyenne Rivers during August 1985 . . . . . . . . . . . . . . . 42

29. Chemical composition of sediment core samples taken from the Cheyenne River arm of Lake Oahe during August 1985 . . . . . . 47

30. Daily mean discharge, in cubic feet per second, for Whi tewood Creek above Whitewood, S. Dak. . . . . . . . . 50

31. Daily mean discharge, in cubic feet per second, for Whitewood Creek above Vale, S. Dak. . . . . . . . . . 52

32. Daily mean discharge, in cubic feet per second, for Belle Fourche River near Sturgis, S. Dak. . . . . . . . . 54

33. Daily mean discharge, in cubic feet per second, for Cheyenne River at Cherry Creek, S. Dak. . . . . . . . 56

34. Chemical analyses of surface-water samples collected at Whitewood Creek above Whitewood, S. Dak. . . . . . . . . 59

35. Chemical analyses of surface-water samples collected at Whitewood Creek above Vale, S. Dak. . . . . . . . . . . . 71

36. Chemical analyses of surface-water samples collected at Belle Fourche River near Sturgis, S. Dak. . . . . . . . . . 83

37. Chemical analyses of surface-water samples collected at Cheyenne River at Cherry Creek, S. Dak. . . . . . . . . . 95

38. Chemical analyses of surface-water samples collected at 14 miscellaneous sites . . . . . . . . . . . . . 105

39. Total chemical analysis of suspended sediment . . . . . . . . 112

40. Water chemistry data for Whitewood Creek/Belle Fourche River, August 1986 . . . . . . . . . . . . . . . . . . 119

41. Arsenic and iron versus filter poresize, Whitewood Creek/Belle Fourche River, August 1986 . . . . . . . . . 121

42. Screen depth and aquifer material for ground-water wells sampled along Whitewood Creek, August 1986 . . . . . . 122

43. Ground-water geochemical data for wells sampled along Whitewood Creek, August 1986 . . . . . . . . . . 124 
Table 44. Specific conductance and temperature measured at the

Sheeler site, Whitewood Creek, during August 1986 . . . . . 126

45. Chemical analysis of water samples collected at the

Sheeler site, Whitewood Creek, during August 1986 . . . . . . 127

46. Photon correlation spectroscopy results for samples collected

at the Sheeler site, Whitewood Creek, during August 1986 . . 129

47. Ground-water chemistry of water collected from seeps along Whitewood Creek, August 1986 . . . . . . . . . . . . 142

48. Arsenic and iron content of iron oxyhydroxide precipitates collected from seeps adjacent to Whitewood Creek,

August 1986 . . . . . . . . . . . . . . . . 143

49. Artificial stream-water composition . . . . . . . . . . . 143

50. Desorption of arsenic from iron oxide precipitates from the seep adjacent to Whitewood Creek at the Berger site; as a function of time and phosphate concentration . . . . . . . 144

51. Desorption of arsenic from iron oxide precipitates from the seep adjacent to Whitewood Creek above Vale; as a function of time and phosphate concentration . . . . . . . . . . . 145

52. Desorption of arsenic from iron oxide precipitates collected from the seep adjacent to Whitewood Creek at the Sheeler site; as a function of time and phosphate concentration . . . . . 146

53. Desorption of arsenic from iron oxide precipitates formed from water from the seep adjacent to Whitewood Creek at the Sheeler site; as a function of time and phosphate concentration .................... 147

54. Estimated periphyton standing crop $\left(P_{b}\right.$ in $\left.\mathrm{g} / \mathrm{m}^{2}\right)$ for four sites along Whitewood Creek sampled in August 1986 . . . . . 149

55. Algal uptake at 24 and 48 hours of arsenate and orthophosphate by living and heat-killed cells using 3 Stichococcus isolates from sites on Whitewood Creek (August 1986)........ 150

56. Relative abundance in 3-5 minute kick samples of selected benthic insects in Whitewood Creek, Belle Fourche River, and the Cheyenne River . . . . . . . . . . . . 153

57. Concentrations of As in benthic insects collected in Whitewood Creek and Belle Fourche River, June 1986 . . . . 155

58. Concentrations of $\mathrm{Fe}$ in benthic insects collected in Whitewood Creek and Belle Fourche River, June 1986 . . . . 156 
For readers who may prefer to use inch-pound units rather than International System (SI) units or International system units rather than inchpound units, the conversion factors for the terms in this report are listed below:

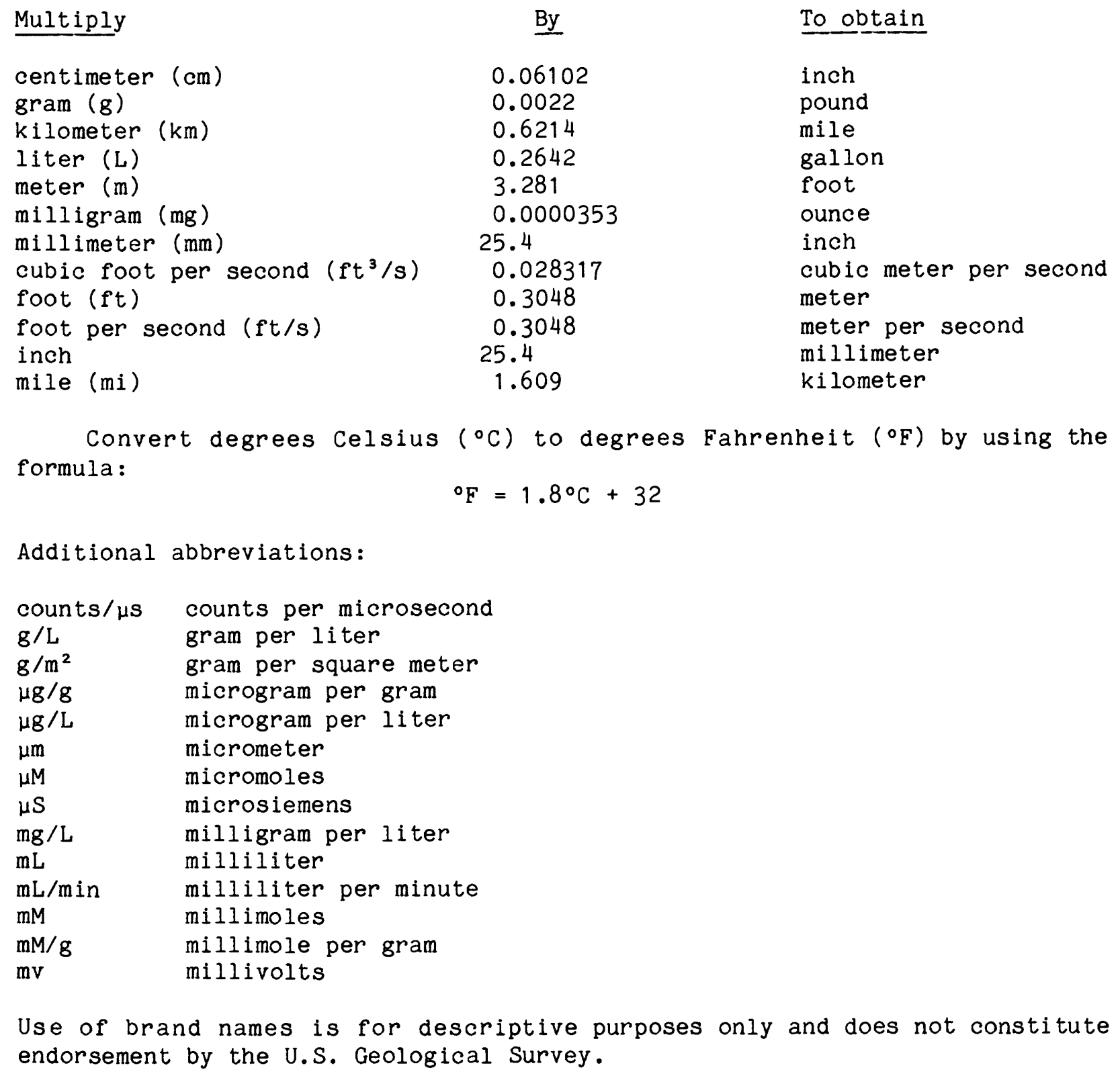

Convert degrees Celsius $\left({ }^{\circ} \mathrm{C}\right)$ to degrees Fahrenheit $\left({ }^{\circ} \mathrm{F}\right)$ by using the formula:

$$
{ }^{\circ} \mathrm{F}=1.8^{\circ} \mathrm{C}+32
$$

Additional abbreviations:

$\begin{array}{ll}\text { counts/us } & \text { counts per microsecond } \\ \mathrm{g} / \mathrm{L} & \text { gram per liter } \\ \mathrm{g} / \mathrm{m}^{2} & \text { gram per square meter } \\ \mu \mathrm{g} / \mathrm{g} & \text { microgram per gram } \\ \mu \mathrm{g} / \mathrm{L} & \text { microgram per liter } \\ \mu \mathrm{m} & \text { micrometer } \\ \mu \mathrm{M} & \text { micromoles } \\ \mu \mathrm{S} & \text { microsiemens } \\ \mathrm{mg} / \mathrm{L} & \text { milligram per liter } \\ \mathrm{mL} & \text { milliliter } \\ \mathrm{mL} / \mathrm{min} & \text { milliliter per minute } \\ \mathrm{mM} & \text { millimoles } \\ \mathrm{mM} / \mathrm{g} & \text { millimole per gram } \\ \mathrm{mv} & \text { millivolts }\end{array}$

Use of brand names is for descriptive purposes only and does not constitute endorsement by the U.S. Geological Survey. 


\title{
U.S. GEOLOGICAL SURVEY APPLIED RESEARCH STUDIES OF THE \\ CHEYENNE RIVER SYSTEM, SOUTH DAKOTA: DESCRIPTION AND \\ COLLATION OF DATA, WATER YEARS 1985-86
}

Edited by Kimball E. Goddard

\begin{abstract}
The Cheyenne River System in western South Dakota has been impacted by the discharge of about 100 million metric tons of gold-mill tailings to Whitewood Creek near Lead, South Dakota. In April 1985, the U.S. Geological Survey initiated an extensive series of research studies to investigate the magnitude of the impact and to define important processes acting on the contaminated sediments present in the system. The report presents all data collected during the 1985 and 1986 water years for these research studies. Some of the data included have been published previously.

Hydrologic, geochemical, and biologic data are available for sites on Whitewood Creek, the Belle Fourche and Cheyenne Rivers, and for the Cheyenne River arm of Lake Oahe. Data complexity varies from routine discharge and water-quality to very complex photon-correlation spectroscopy and energydispersive x-ray analysis. Methods for sample collection, handling and preservation, and laboratory analysis are also presented. No interpretations or complex statistical summaries are included.
\end{abstract}

\section{INTRODUCTION}

Beginning in the 1960's, people became more aware of the serious environmental hazard and economic damage that could result from the continued use of our Nation's rivers and streams for waste disposal. Resulting legislation, such as the Federal Water Pollution Control Act Amendments of 1972 ( $P$.L. 92-500), the Toxic Substances Control Act of 1976 (P.L. 97-469), and the National Pollutant Discharge Elimination System (NPDES) Permit Program provided the impetus for a nationwide cleanup of rivers and streams. As a result, the quality of the Nation's rivers and streams has improved substantially in several respects, reflected by increased dissolved-oxygen concentrations and decreased bacterial populations, that are largely attributable to measures for controlling point-source discharges and the construction or improvement of wastewater-treatment facilities. However, numerous other water-quality issues remain and the focus of concern is shifting from sewage disposal to control of potentially more hazardous wastes such as toxic metals and synthetic organic compounds.

Recent studies on the geochemical reactions of toxic metals and synthetic organic compounds in river and stream systems have demonstrated that these constituents are commonly associated with river or stream sediments. The extremely low solubilities of some metals, such as mercury and lead, in normal 
stream water and the affinity of many synthetic organic compounds for sediments, generally result in very small to undetectable dissolved concentrations of these constituents in surface water. However, these constituents may accumulate in bottom sediments in sufficiently large concentrations so as to allow uptake by aquatic plants, benthic organisms, or bottom-feeding fish. Although toxic constituents concentrated in bottom sediments may be isolated from the environment for long periods by burial, they can be exposed and transported during floods. It is clear that the adsorption of hazardous constituents onto channel bottom sediments exacerbates the difficulty of understanding the processes responsible for the movement and fate of these constituents in river systems.

In 1985, the U.S. Geological Survey initiated the Toxic Substances in Surface Waters and Sediments Thrust Program. The program is designed to: (1) define the occurrence of toxic substances in water, sediment, and biota, (2) conduct research on the transport of toxic substances, and (3) conduct research to improve sampling and analytical methods. In April 1985, the Cheyenne River basin in western South Dakota was selected for study. Investigative efforts at the study site are cooperatively divided between District and National Research Program personnel.

\section{Problem}

About 100 million metric tons of finely ground gold-mill tailings were discharged into Whitewood Creek near Lead, South Dakota, between 1876 and 1977. The tailings contained substantial concentrations of arsenic, mercury, and other trace elements resulting in widespread contamination of the alluvial sediments along Whitewood Creek and the Belle Fourche and Cheyenne Rivers (figs. 1 and 2). The primary contaminant is arsenic derived from, or still present as, the sulfide mineral arsenopyrite (FeAsS), a gangue mineral common to the gold-bearing deposits at Lead. The trace elements antimony, cadmium, copper, iron, manganese, mercury, and silver also are associated with the mill tailings and are present in the contaminated alluvial sediments.

The gross contamination of Whitewood Creek and the downstream $r i v e r s$ was alleviated by the discontinuance of tailings discharge in December 1977. However, the huge deposits of contaminated sediments present along Whitewood Creek and the Belle Fourche and Cheyenne Rivers continues to degrade surface water and ground water in the affected portion of the Cheyenne River basin. Because the contaminated sediment deposits were formed by natural surface flow, the contaminated sediments are subject to resuspension and downstream movement, particularly during periods of high discharge when sediment enters the flow from streambed scouring, bank collapse, or by input from overland runoff. Suspended-sediment samples collected from Whitewood Creek and the Belle Fourche and Cheyenne Rivers since 1977 continue to have unusually large concentrations of total arsenic, iron, and manganese. Dissolved arsenic, thought to be derived from alluvial ground-water and desorbed from streambed material, is present in substantial concentrations in Whitewood creek. Portions of the alluvial aquifers along Whitewood Creek and the Belle Fourche River are contaminated by leachate derived from overlying contaminated sediment deposits. 


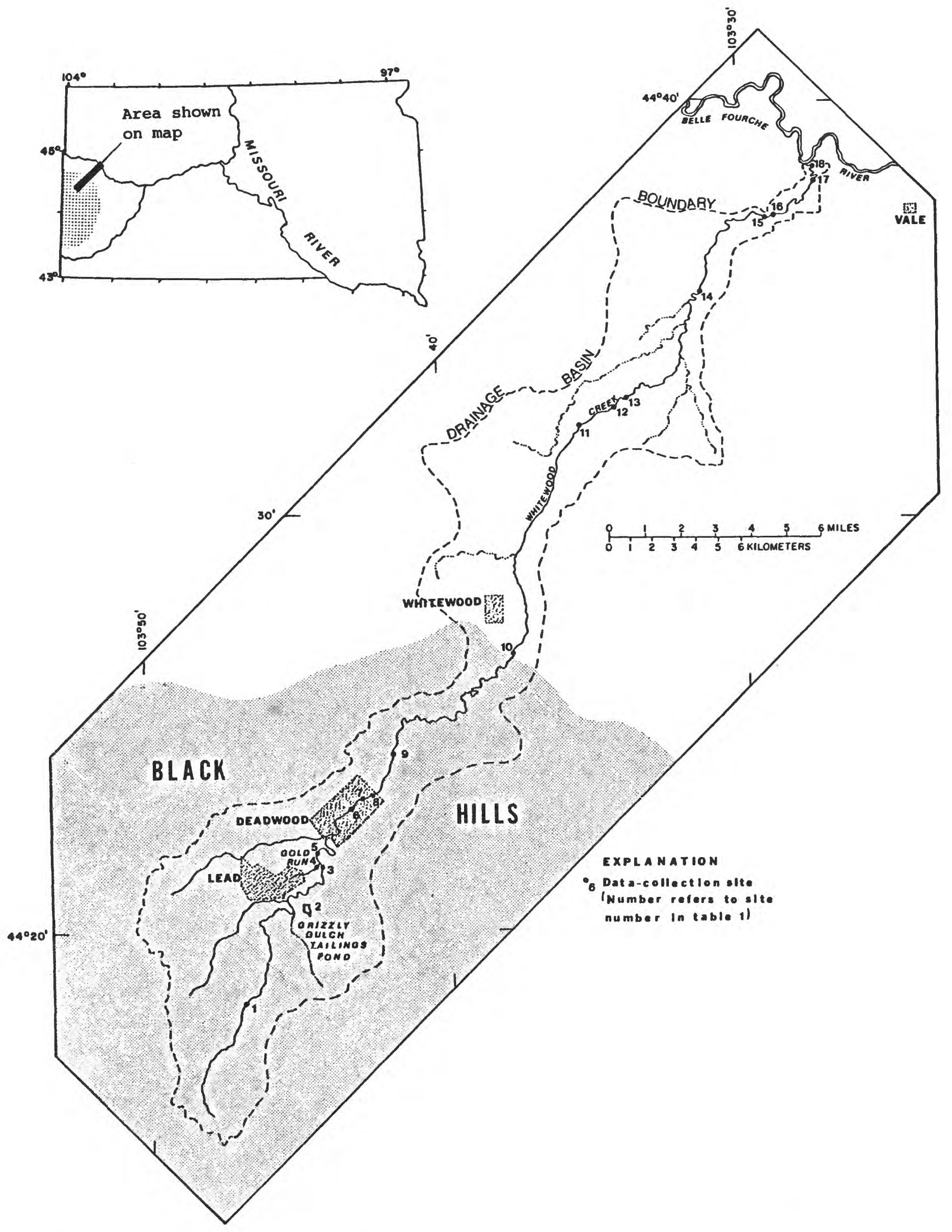

Figure 1.--Data-collection sites within the Whitewood Creek drainage basin. 


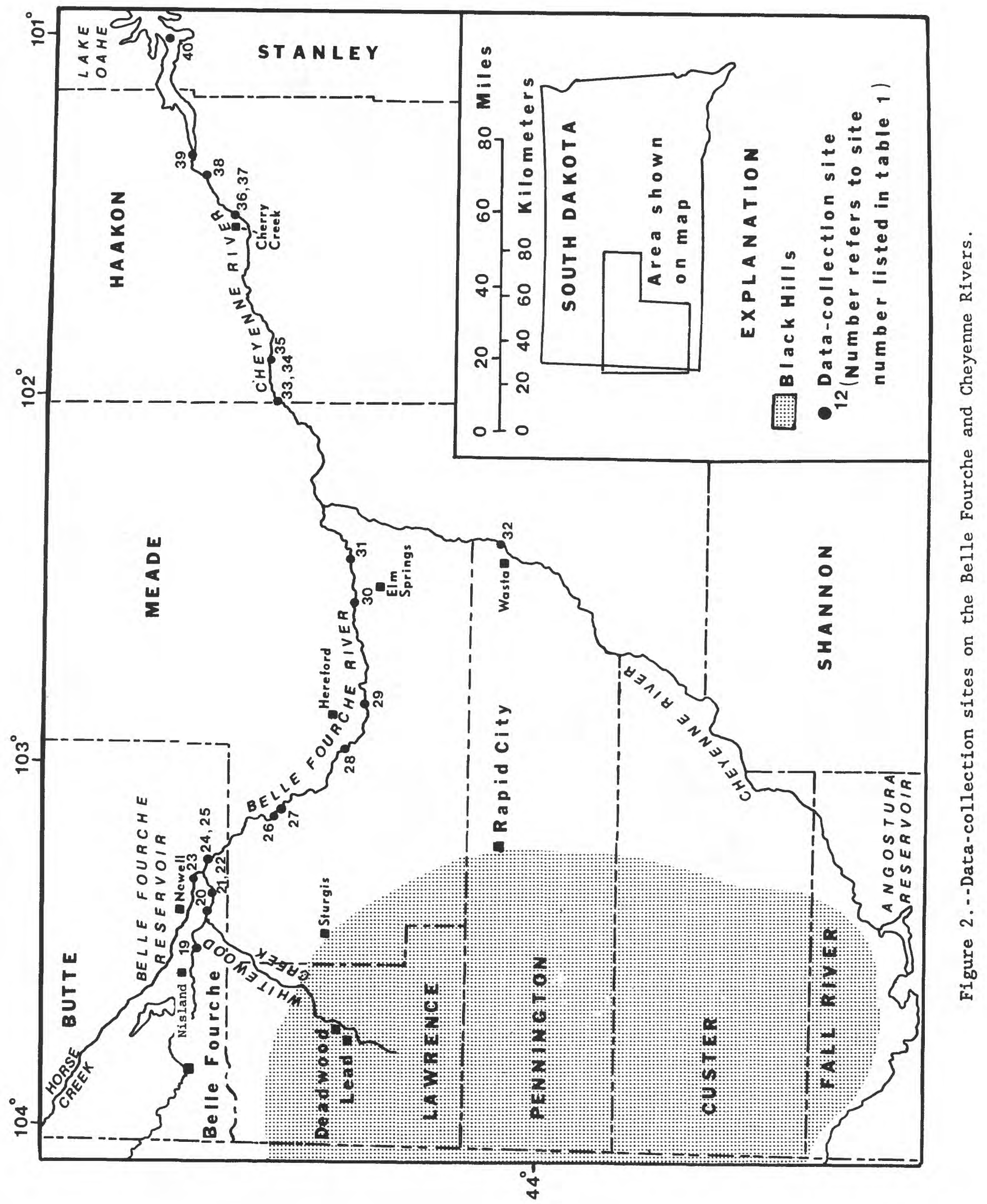


Table 1.--Names of data-collection sites discussed in text. Location of sites 1 to 18 shown in figure 1 ; location of sites 19-40 shown in figure 2

$[Q$, continuous discharge; SW, surface-water chemistry; $S$, suspendedsediment chemistry; BM, bottom-material chemistry; AS, alluvialsediment chemistry; GW, ground-water chemistry; $B$, biologic]

\begin{tabular}{|c|c|c|c|}
\hline $\begin{array}{c}\text { Site } \\
\text { number }\end{array}$ & Si te name & $\begin{array}{l}\text { Date } \\
\text { type }\end{array}$ & $\begin{array}{l}\text { Report } \\
\text { chapter }\end{array}$ \\
\hline 1 & Whitewood Creek above Lead & $\mathrm{SW}, \mathrm{BM}, \mathrm{B}$ & $C, E, J$ \\
\hline 2 & Grizzly Gulch tailings pond & AS & A \\
\hline 3 & Whi tewood Creek above Gold Run & SW, BM & $C, E$ \\
\hline 4 & Gold Run at mouth & SW & $\mathrm{E}$ \\
\hline 5 & Whitewood Creek below Gold Run & SW, B & $E, J$ \\
\hline 6 & Whi tewood Creek at Deadwood & SW, B & $E, F, J$ \\
\hline 7 & Whitewood Creek above Deadwood STP & SW, B & $E, J$ \\
\hline 8 & Whitewood Creek below Deadwood STP & SW, B & $\mathrm{E}, \mathrm{J}$ \\
\hline 9 & Whitewood Creek below Deadwood & $S W, B M, B$ & $C, E, J$ \\
\hline 10 & Whitewood Creek above Whi tewood & $Q, S W, S, B M$ & $A, C, E, F$ \\
\hline 11 & Whitewood Creek near Whitewood & $\mathrm{BM}$ & $\mathrm{C}$ \\
\hline 12 & Whi tewood transect one (WW1) & AS & $\mathrm{B}$ \\
\hline 13 & Berger site & SW, AS, GW, B & $A, E, F, G, I, J$ \\
\hline 14 & Whitewood Creek at Custer Camp & MV & $\mathrm{C}$ \\
\hline 15 & Whitewood Creek above Vale & $\mathrm{Q}, \mathrm{SW}, \mathrm{S}, \mathrm{BM}, \mathrm{B}$ & $A, C, E, I, J$ \\
\hline 16 & Whitewood transect two (WW2) & AS & $\mathrm{B}$ \\
\hline 17 & Sheeler site & $S W, A S, G W, B$ & $A, E, F, G, H, I, J$ \\
\hline 18 & Whitewood Creek near mouth & $\mathrm{B}$ & $\mathrm{J}$ \\
\hline 19 & Belle Fourche River below Nisland & SW, S, BM, B & $A, C, E, J$ \\
\hline 20 & Belle Fourche River at Vale & $\mathrm{BM}$ & $\mathrm{C}, \mathrm{F}$ \\
\hline 21 & Belle Fourche transect one (BF1) & AS & $\mathrm{B}$ \\
\hline 22 & Belle Fourche River at old Vale bridge & $\mathrm{BM}$ & C \\
\hline 23 & Horse Creek above Vale & SW, $S$ & $\mathrm{E}$ \\
\hline 24 & Belle Fourche River below Vale & $\mathrm{S}$ & $\mathrm{E}$ \\
\hline 25 & Belle Fourche transect two (BF2) & AS & B \\
\hline 26 & Belle Fourche River near Sturgis & $Q, S W, S, B M$ & $A, C, E$ \\
\hline 27 & Belle Fourche transect three (BF3) & AS & $\mathrm{B}$ \\
\hline 28 & Belle Fourche River near Hereford & SW, $S$ & A \\
\hline 29 & Belle Fourche transect four (BF 4$)$ & AS & B \\
\hline 30 & Belle Fourche River near Elm Springs & SW, S, BM & $A, C, E$ \\
\hline 31 & Belle Fourche transect five (BF5) & AS & B \\
\hline 32 & Cheyenne River near Wasta & SW, S & $E$ \\
\hline 33 & Cheyenne River transect one (CR1) & AS & B \\
\hline 34 & Cheyenne River near Plainview & $\mathrm{BM}$ & C \\
\hline 35 & Cheyenne River at SD Hwy 34 bridge & $\mathrm{B}$ & $\mathrm{J}$ \\
\hline 36 & Cheyenne River transect two (CR2) & AS & $\mathrm{B}$ \\
\hline 37 & Cheyenne River at Cherry Creek & $\mathrm{Q}, \mathrm{SW}, \mathrm{S}, \mathrm{BM}$ & $C, E$ \\
\hline 38 & Cheyenne River at Carlin Flat & SW, S & $E$ \\
\hline 39 & Cheyenne River at SD Hwy 63 bridge & $\mathrm{BM}$ & $\mathrm{C}$ \\
\hline 40 & Cheyenne River arm of Lake Oahe & AS & $\mathrm{D}$ \\
\hline
\end{tabular}


Although the magnitude of arsenic and other trace element contamination in the Cheyenne River basin has been documented by previous investigations, many process-oriented questions remain. It is apparent that the original source of arsenic contamination was arsenopyrite present in the tailings discharge at Lead. The current source of contamination is much less obvious because of the dispersed nature of the contaminated sediments. How much of the originally discharged material remains in the basin, how much of the arsenic mass still exists as arsenopyrite, what mechanisms are responsible for arsenic transport, and what are the annual rates of downstream transport, are all questions that remain to be answered. In addition, although it has been documented that dissolved arsenic is present in substantial concentrations in some ground water and in Whitewood Creek, the source of the dissolved arsenic, and the frators controlling the dissolved arsenic concentration, are also not currently understood.

\section{Purpose and Scope}

This report presents basic data collected in the 1985 and 1986 water years during U.S. Geological Survey research studies of the tailings contamination in the Cheyenne River basin. The report was prepared so that methodology and data obtained during a wide variety of research studies would be easily available. No interpretations or complex statistical summaries are included. Persons interested in interpretation of these data should contact individual authors who have prepared interpretive reports or papers for most chapters. Some of the data included in this report have been previously published. 


\title{
Chapter A. Geochemical Characteristics of Contaminated Sediments from Deposits along Whitewood Creek
}

By Thomas E. McKallip, Kimball E. Goddard, Ray W. Wuolo, and Reinhard W. Leinz

\author{
PHYSICAL AND CHEMICAL CHARACTERISTICS
}

Sample Collection and Handling Procedures

Four sediment samples, considered to be representative of the contamination problem in the Whitewood Creek system, were collected in early April 1986. Two of the samples were mill tailings collected from the Grizzly Gulch tailings disposal pond while the second pair were highly contaminated sediments collected from deposits along Whitewood Creek. The sample number, description, and location are listed in table 2. The two contaminated sediment samples were collected from sites at which there are additional hydrologic and geochemical data available.

Sediment samples were collected by hand using a small epoxy-covered metal trowel. Samples were placed directly into acid-rinsed wide-mouth gallon bottles. The samples were air-dried on plastic plates, then stored at room temperature in the original field-sample containers until analysis.

Table 2.--Sample number, source, description, and location for sediment samples collected in April 1986

\begin{tabular}{|c|c|c|c|}
\hline $\begin{array}{l}\text { Sample } \\
\text { number }\end{array}$ & Source & Description & Location \\
\hline 1 & $\begin{array}{l}\text { Grizzly Gulch } \\
\text { tailings pond }\end{array}$ & Black sand & $\begin{array}{l}44019 \cdot 40 " 103^{\circ} 44^{\prime} 15^{\prime \prime} \\
\text { T4N R3E Sec. } 3 \operatorname{SE}_{4}^{\frac{1}{4} S E^{\frac{1}{4}}}\end{array}$ \\
\hline 2 & $\begin{array}{l}\text { Grizzly Gulch } \\
\text { tailings pond }\end{array}$ & Gray fine silt & 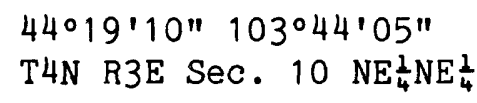 \\
\hline 3 & $\begin{array}{l}\text { Whitewood Creek } \\
\text { (Berger site) }\end{array}$ & $\begin{array}{l}\text { Blue-gray very } \\
\text { fine silt }\end{array}$ & $\begin{array}{l}44^{\circ} 33^{\prime} 02^{\prime \prime} 103^{\circ} 32^{\prime} 55^{\prime \prime} \\
\text { T7N R5E Sec. } 19^{\prime} \mathrm{NE}_{4}^{\frac{1}{4} S E_{4}}\end{array}$ \\
\hline 4 & $\begin{array}{l}\text { Whitewood Creek } \\
\text { (Sheeler site) }\end{array}$ & $\begin{array}{l}\text { Red-orange } \\
\text { sandy silt }\end{array}$ & $\begin{array}{l}44^{\circ} 38^{\prime} 24^{\prime \prime} 103^{\circ} 27^{\prime} 23^{\prime \prime} \\
\text { T8N R5E Sec. } 24^{N} \mathrm{NW}_{4} \mathrm{SE}_{\frac{1}{4}}\end{array}$ \\
\hline
\end{tabular}

Analytical Procedures and Results

Total digestion analysis was performed on subsamples from the bulk samples by J. Motooka of the U.S. Geological Survey, Geologic Division, Branch of Exploration Geochemistry, Arvada, Colorado. Digestion was by $\mathrm{HF} / \mathrm{HClO}_{4} / \mathrm{HNO}_{3}$ acid with detection by inductively coupled plasma atomic emission spectrometer. Analytical results are listed in table 3 . 


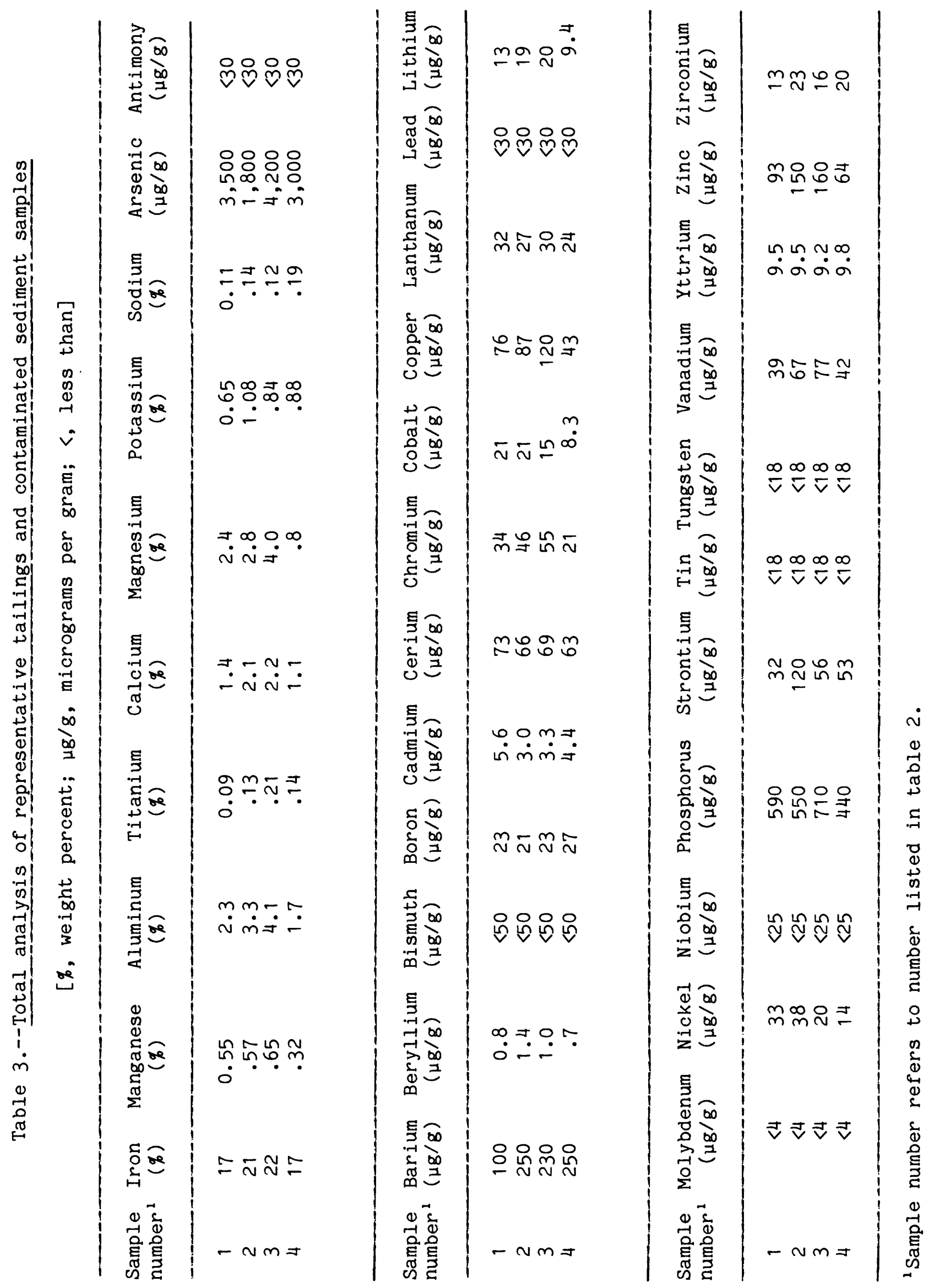


Subsamples from the bulk samples were further split by specific gravity and magnetic properties by John Unruh, U.S. Geological Survey, Geologic Division, Arvada, Colorado. Samples were washed first in water to remove clay-size particles which will not gravity settle in bromoform. Samples were then split into light (specific gravity less than 2.8) and heavy (specific gravity greater than 2.8) fractions using bromoform flotation. The heavy fraction was further split into magnetic, weakly magnetic, and non-magnetic fractions. The resultant splits underwent semi-quantitative analysis using D. C. Arc spectrography. The results of the separations and the analytical results are listed in table 4. The elements antimony, bismuth, cadmium, molybdenum, niobium, thorium, $t$ in, tungsten, and zinc were included in the analysis but are not listed in table 4 because they were below the detection limits.

Subsamples from the bulk samples underwent sequential digestion and arsenic analysis by R. F. Sanzolone, U.S. Geological Survey, Geologic Division, Arvada, Colorado. The sequential digestion procedure involved attacking the sample with increasing strength acids to determine what fraction of the sample contained arsenic. Arsenic concentrations were determined using an inductively coupled plasma atomic emission spectrometer. The sequential digestion procedure used and the analytical results are listed in table 5.

\section{ADSORPTION/DESORPTION CHARACTERISTICS}

\section{Sample Collection and Handling Procedures}

Sediments for adsorption/desorption studies were collected from the streambank and flood plain of Whitewood Creek at a location considered typical of the lower portion of the drainage basin (Wuolo, 1986). The location, commonly referred to as the Berger site by previous investigators, is shown in figure 1 and listed in table 1. The Berger site has been extensively studied (Stach and others, 1978; Cherry and others, 1984; and Goddard, in press). Large amounts of additional hydrologic and geochemical data are available.

In October 1985, four large samples were collected at the Berger site for the characterization of physical and chemical properties and adsorption/ desorption. Three samples of contaminated sediment ( $\mathrm{Tc}-1, \mathrm{Tc}-2, \mathrm{Tc}-3)$ and one of uncontaminated sediment $(\mathrm{AC}-1)$ were obtained using an acid-rinsed plastic spatula. About 7 inches of surface material was removed before collection. Samples were stored in large sealable plastic bags and frozen for storage. In order to simulate field conditions in the column leaching experiments, seven additional samples were collected adjacent to the large adsorption/desorption samples. Permeability samples were collected by driving a shelby-tube sampler vertically (flood plain) or horizontally (streambank) into sediments. 


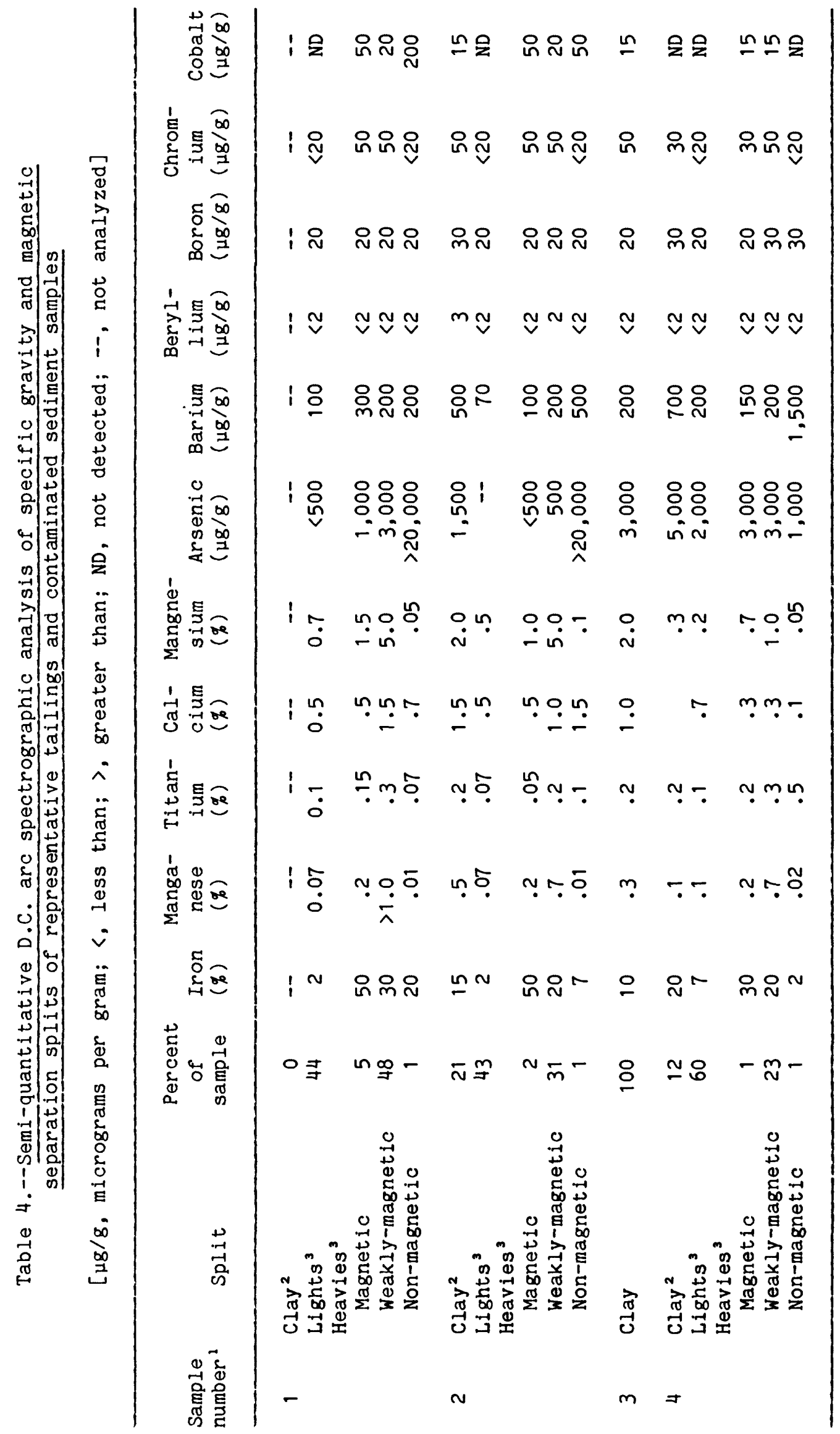




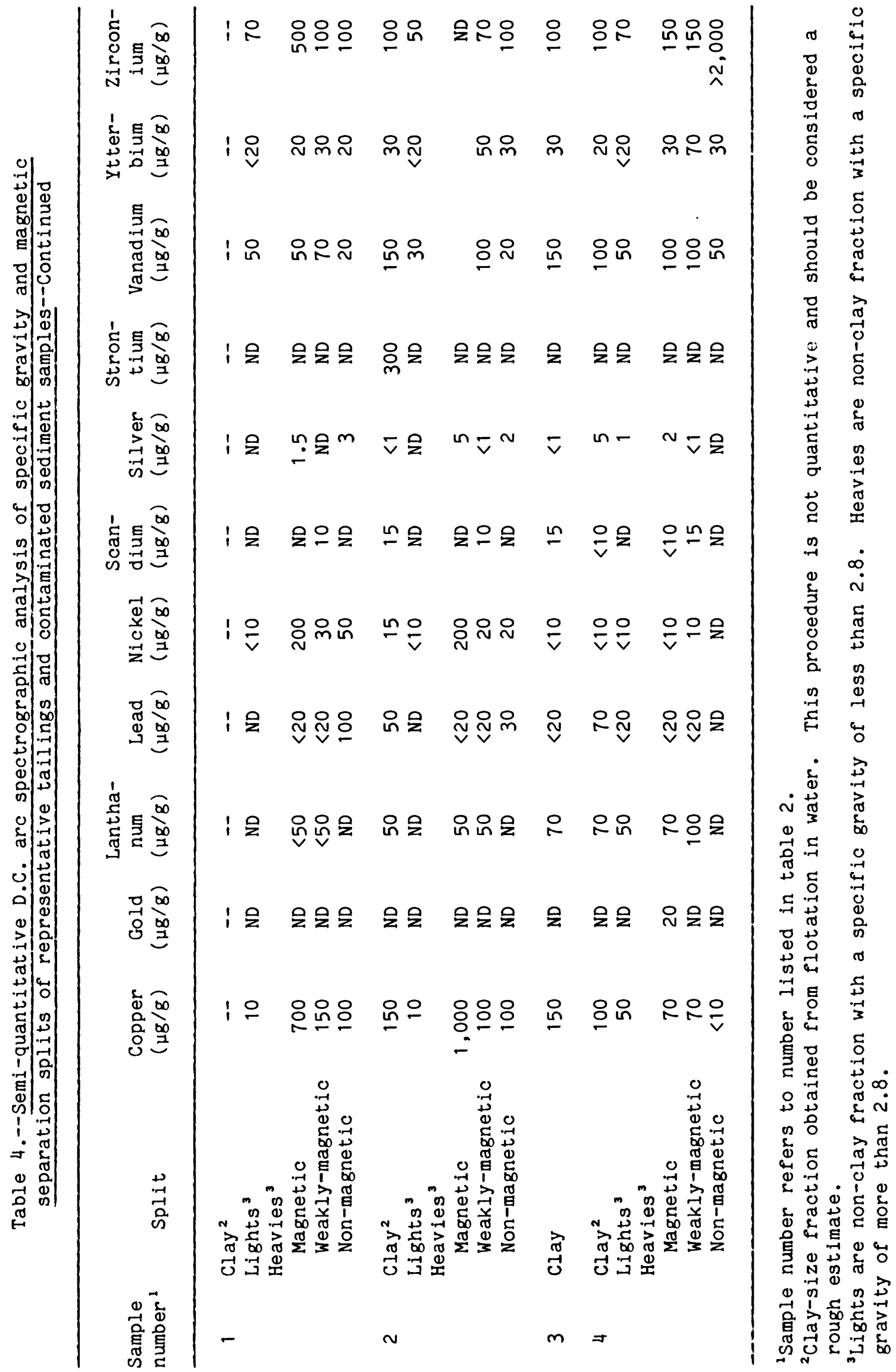


Table 5.--Sequential digestion procedure and arsenic concentrations measured in representative tailings and contaminated sediment samples

\section{$[<$, less than $]$}

\begin{tabular}{|c|c|c|c|c|c|}
\hline \multirow[b]{3}{*}{ Digestion } & \multirow{3}{*}{$\begin{array}{l}\text { Recovered } \\
\text { fraction }\end{array}$} & \multicolumn{4}{|c|}{$\begin{array}{c}\text { Arsenic concentration in fraction } \\
\text { (micrograms per gram) }\end{array}$} \\
\hline & & \multicolumn{4}{|c|}{ Sample number ${ }^{1}$} \\
\hline & & 1 & 2 & 3 & 4 \\
\hline $0.1 \mathrm{M} \mathrm{KH}_{2} \mathrm{PO}_{4}$ & $\begin{array}{l}\text { Water soluble/ } \\
\text { exchangeable }\end{array}$ & $<250$ & $<250$ & $<250$ & 250 \\
\hline $4 \mathrm{~N} \mathrm{HCl}$ & Oxide/carbonate & $<250$ & $<250$ & $<250$ & 3,000 \\
\hline $\mathrm{KClO}_{3} / \mathrm{HCl}$ & Sulfide & 3,300 & 1,600 & 3,400 & $<250$ \\
\hline $\mathrm{HF} / \mathrm{HClO}_{4} / \mathrm{HNO}_{3}$ & Silicates & $<250$ & $<250$ & $<250$ & $<250$ \\
\hline $\mathrm{HF} / \mathrm{HClO}_{4} / \mathrm{HNO}_{3}$ & Total & 3,700 & 1,600 & 3,700 & 3,000 \\
\hline
\end{tabular}

${ }^{1}$ Sample number refers to number listed in table 2.

\section{Analys is of Physical and Chemical Properties}

Mineralogy of these samples was determined by $x$-ray diffraction analysis of freeze-dried splits. Results are reported in weight fractions and variances for major minerals (table 6) and total oxide contents (table 7).

Grain-size analysis of sample splits of $200 \mathrm{~g}$ of $\mathrm{Ac}-1, \mathrm{Tc}-1, \mathrm{Tc}-2$, and Tc-2 was done using the sample pretreatment procedure outlined by Bodman (1928). The greater than 62-um fraction was dried and mechanically separated by sieving. The less than 62-um fraction was separated down to $2 \mu \mathrm{m}$ using the pipet method outlined by Friedman and Johnson (1982). The results of grainsize analysis are given in table 8.

Permeabilities were determined by converting the shelby sampler into a permeameter. Hydrologic conductivity was calculated using variations of Darcy's law as proposed by Fetter (1980). Porosities were determined by extruding saturated samples, weighing them, and subtracting the weight of the sample after it was oven dried for 26 hours at $125^{\circ} \mathrm{C}$. Permeability and porosity results are given in table 9. 


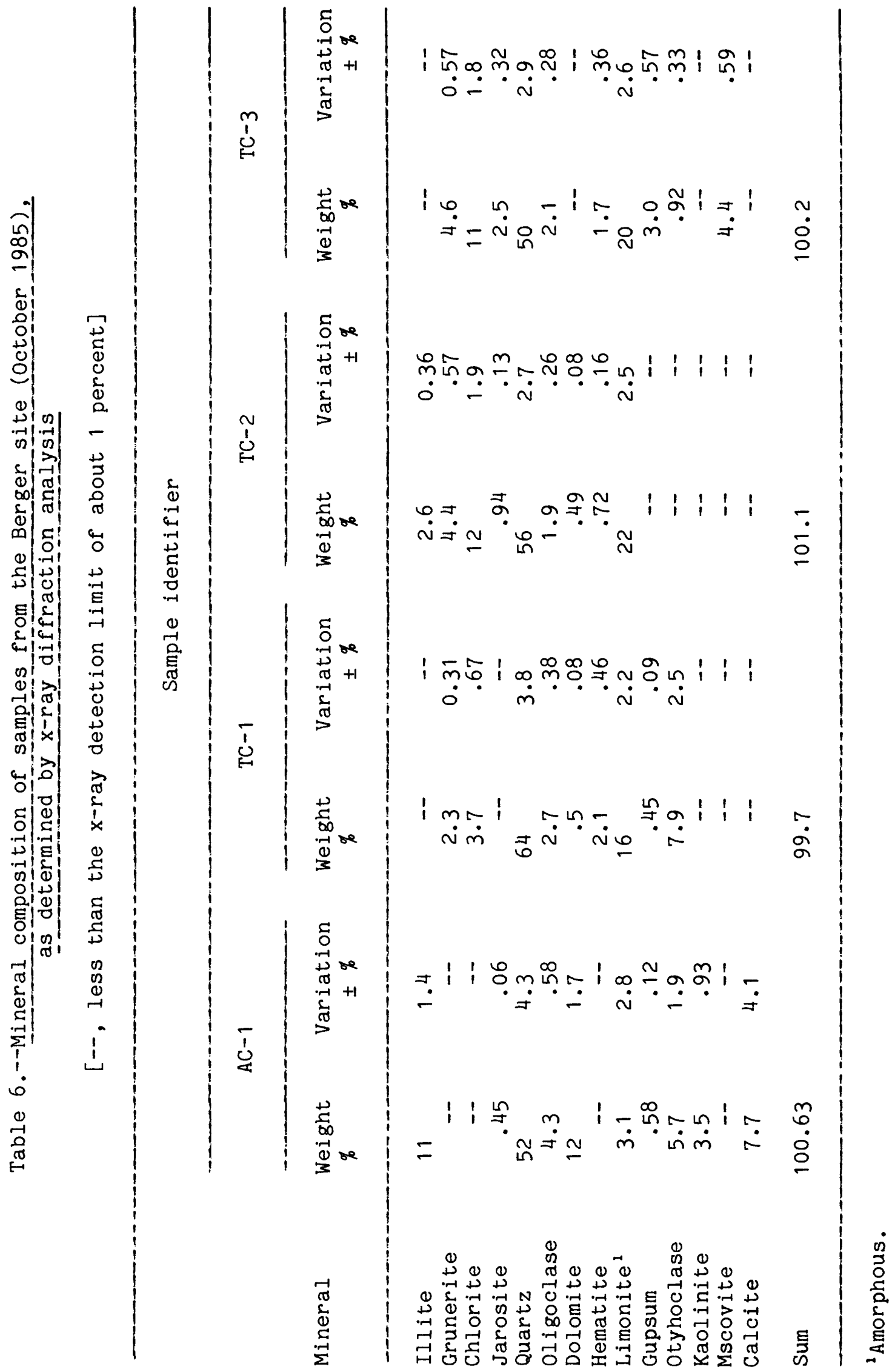


Table 7.--Weight percent of major oxides for samples from the Berger site, as determined by $x$-ray diffraction analysis (0ctober 1985)

\begin{tabular}{|c|c|c|c|c|}
\hline \multirow{3}{*}{ Oxide } & \multicolumn{4}{|c|}{ Sample identifier } \\
\hline & $A C-1$ & $\mathrm{TC}-1$ & $\mathrm{TC}-2$ & $\mathrm{TC}-3$ \\
\hline & $\begin{array}{c}\text { Weight } \\
\%\end{array}$ & $\underset{\%}{\text { Weight }}$ & $\underset{\%}{\text { Weight }}$ & $\underset{q}{\text { Weight }}$ \\
\hline $\mathrm{SiO}_{2}$ & 65 & 73 & 64 & 60 \\
\hline $\mathrm{Al}_{2} \mathrm{O}_{3}$ & 6.7 & 3.4 & 4.2 & 4.9 \\
\hline $\mathrm{NO}_{2} \mathrm{O}$ & .64 & .43 & .23 & .36 \\
\hline $\mathrm{K}_{2} \mathrm{O}$ & 1.6 & 1.0 & .24 & .63 \\
\hline CaO & 8.1 & .53 & .42 & 1.3 \\
\hline $\mathrm{FeO}$ & .45 & 1.4 & 3.3 & $3 \cdot 3$ \\
\hline $\mathrm{Fe}_{2} \mathrm{O}_{3}$ & 3.2 & 15 & 18.7 & 19 \\
\hline $\mathrm{MgO}$ & 2.6 & 1.0 & 3.0 & 2.8 \\
\hline $\mathrm{H}_{2} \mathrm{O}$ & 1.9 & 2.8 & 4.7 & 5.2 \\
\hline $\mathrm{CO}_{2}$ & 8.9 & .23 & .23 & .02 \\
\hline $\mathrm{SO}_{3}^{2}$ & .41 & .20 & .30 & 2.2 \\
\hline Mno & .13 & .12 & .36 & .35 \\
\hline $\mathrm{TiO}_{2}$ & .06 & .02 & .04 & .04 \\
\hline $\mathrm{PbO}$ & 0 & 0 & 0 & 0 \\
\hline $\mathrm{NiO}$ & .01 & 0 & 0 & 0 \\
\hline CuO & 0 & 0 & 0 & 0 \\
\hline $\mathrm{V}_{2} \mathrm{O}_{3}$ & 0 & 0 & 0 & 0 \\
\hline $\mathrm{P}_{2} \mathrm{O}_{5}$ & .01 & .05 & .06 & .06 \\
\hline $\mathrm{Cr}_{2} \mathrm{O}_{3}$ & 0 & 0 & 0 & .03 \\
\hline $\mathrm{As}_{2} \mathrm{O}_{3}$ & 0 & 0 & 0 & 0 \\
\hline $\mathrm{MnO}_{2}$ & 0 & 0 & 0 & 0 \\
\hline $\mathrm{BaO}^{2}$ & 0 & .01 & 0 & .02 \\
\hline Sro & 0 & 0 & 0 & 0 \\
\hline $\mathrm{Li}_{2} \mathrm{O}$ & 0 & 0 & 0 & 0 \\
\hline $\mathrm{Zno}$ & 0 & 0 & 0 & 0 \\
\hline Sum & 99.9 & 99.9 & 99.9 & 99.9 \\
\hline
\end{tabular}


Table 8.--Grain-size analysis of samples from the Berger site (October 1985)

Mass of size fraction for sample identifier (grams)

\begin{tabular}{|c|c|c|c|c|c|}
\hline \multirow{2}{*}{ Micrometers } & \multirow[b]{2}{*}{ Method } & & & & \\
\hline & & $A C-1$ & $\mathrm{TC}-1$ & $\mathrm{TC}-2$ & $\mathrm{TC}-3$ \\
\hline \multicolumn{6}{|l|}{1,000} \\
\hline & Sieve & 0.00 & 1.07 & 0.15 & 0.00 \\
\hline \multicolumn{6}{|l|}{710} \\
\hline \multicolumn{6}{|l|}{500} \\
\hline & do. & .00 & 9.69 & .40 & .08 \\
\hline \multicolumn{6}{|l|}{420} \\
\hline \multicolumn{6}{|l|}{300} \\
\hline & do. & 1.02 & 35.84 & .82 & .93 \\
\hline \multicolumn{6}{|l|}{210} \\
\hline \multirow{2}{*}{177} & 00 & 2.93 & 31.23 & 2.09 & 1.07 \\
\hline & do. & 3.16 & 11.03 & 2.57 & 2.02 \\
\hline 149 & do. & 5.45 & 8.52 & 4.02 & 3.42 \\
\hline \multicolumn{6}{|l|}{125} \\
\hline \multirow{2}{*}{74} & do. & 2.64 & 2.72 & 11.72 & 5.75 \\
\hline & do. & 40.73 & 9.24 & 38.38 & 28.65 \\
\hline \multicolumn{6}{|l|}{63} \\
\hline \multirow{2}{*}{31} & Pipette & 68.5 & 13.5 & 24.0 & 24.0 \\
\hline & do. & 49.0 & 9.5 & 20.0 & 7.5 \\
\hline 15.6 & do. & 44.5 & 7.0 & 14.5 & 5.5 \\
\hline 7.8 & & & 11 & & \\
\hline \multirow[t]{2}{*}{3.9} & do. & $4<. U$ & 4.5 & 14.5 & 4.0 \\
\hline & do. & 27.0 & 4.5 & 9.0 & 3.5 \\
\hline 2.0 & do. & 8.0 & 3.0 & 8.0 & 1.0 \\
\hline
\end{tabular}


Table 9.--Summary of permeability test results of samples from the Berger site (october 1985 )

\begin{tabular}{|c|c|c|c|}
\hline Sediment type & Sampling orientation & $\begin{array}{l}\text { Porosity } \\
\text { (percent) }\end{array}$ & $\begin{array}{l}\text { Hydraulic conductivity } \\
\text { (feet per second) }\end{array}$ \\
\hline Uncontaminated & Horizontal & 49 & $1.90 \times 10^{-7}$ \\
\hline Contaminated & do. & 37 & $4.92 \times 10^{-5}$ \\
\hline Do. & do. & 52 & $1.80 \times 10^{-7}$ \\
\hline Do. & do. & 56 & $3.28 \times 10^{-5}$ \\
\hline Do. & Vertical & 47 & $1.97 \times 10^{-6}$ \\
\hline Do. & do. & 61 & $2.46 \times 10^{-4}$ \\
\hline Do. & do. & 59 & $5.91 \times 10^{-6}$ \\
\hline
\end{tabular}

\section{Determination of Adsorption/Desorption Characteristics}

In preparation for batch experiments, freeze-dried splits of $A c-1$ and Tc-2 were sieved through a 500-um polypropolene screen, and the coarser fraction was discarded. Samples were handled with plastic spatulas. The average major ion concentrations in the alluvial ground water are known from previous studies by Cherry and others (1984), Fox Associates (1984), and Goddard (in press). These ion concentrations were simulated using reagentgrade compounds. Stock solution for As+3 and As+5 were prepared with reagent grade $\mathrm{NaAsO}_{2}$ and $\mathrm{Na}_{2} \mathrm{HAsO}_{4} \cdot 7 \mathrm{H}_{2} \mathrm{O}$ daily in bottles rinsed and soaked in 3 percent nitric acid overnight.

Reaction vessels were 250-mL polypropylene bottles that had been acid rinsed, double rinsed in distilled water, and soaked overnight in distilled water prior to each use. Solid and liquid were combined in the reaction vessel. An aliquot of the appropriate arsenic species was pipetted into the vessel to obtain the desired arsenic concentration. The $\mathrm{pH}$ of the contents of each reaction vessel was titrated to the desired $\mathrm{pH}$ by the addition of a small aliquot of 10 percent $\mathrm{NaOH}$ or 10 percent $\mathrm{HCL}$ and the vessel was shaken for one hour in a wrist-action shaker. The $\mathrm{pH}$ was remeasured and adjusted as necessary. The unadjusted redox potential was recorded with a combination platinum electrode, but redox potential is reported with respect to a standard hydrogen electrode. Temperature also was recorded. 
Redox potential and $\mathrm{pH}$ were measured in a glove bag filled with nitrogen gas to prevent atmospheric oxygen from entering the sample. The solution was filtered through a $0.45-\mu m$ cellulose-nitrate membrane filter into 250-mL, acid-rinsed bottles using a peristaltic pump and preserved with nitric-acid for arsenic analysis. Samples used for sulfate analysis were filtered into bottles not rinsed with acid and were not preserved with acid.

All filtered samples were sent to the U.S. Geological Survey Water Resources Division Central Laboratory in Arvada, Colorado, for arsenic and sulfate analysis. Arsenic analyses were by atomic-adsorption spectroscopy following hydride generation. Sulfate analyses were by turbidimetric methods. Detection limits for arsenic and sulfate were $1 \mu \mathrm{g} / \mathrm{L}$ and $0.2 \mathrm{mg} / \mathrm{L}$ respectively (Feltz and Anthony, 1985).

Adsorption characteristics of the reaction vessels were determined by using $200 \mathrm{~mL}$ of simulated water as a blank and shaking the sample for 24 hours at fixed, various pHs (table 10). Based on previous work by olsen and Wat anabe (1957) and Frost and Griffen (1977), a sediment-to-liquid ratio of $1: 20$ was decided upon for the remaining batch experiments. The effect of varying the solid-to-liquid ratio is shown in table 11 .

Batch experiments were run with contaminated sediment (Tc-2) at varying durations to determine the effect equilibrium time on adsorption. The $\mathrm{pH}$ of each sample was adjusted to $7.0 \pm 0.3$ prior to shaking. The results are contained in table 12 .

The effect of $\mathrm{pH}$ on adsorption was explored for both uncontaminated and contaminated sediments at four different concentrations of As (III) and As (V). Solid and simulated water were allowed to equilibrate for about 1 hour before the $\mathrm{pH}$ was adjusted. A final equilibration period of 1 hour was allowed and the $\mathrm{pH}$ was "fine-tuned" to $\pm 0.3 \mathrm{pH}$ units from the desired pH values of 3.5 , $6.5,9.5$, and 12.5 .

Various initial $\mathrm{As}^{+}{ }^{3}$ and $\mathrm{As}^{+5}$ concentrations were employed. Shake time was 24 hours. Initial $\mathrm{SO}_{4}$ concentration was $656 \mathrm{mg} / \mathrm{L}$. The solution's $\mathrm{pH}$, Eh, and temperature were measured prior to filtration and the results are listed in tables 13 and 14 .

The effect of sulfate concentration on arsenic adsorption was examined in batch experiments with both the uncontaminated sediment (AC-1) and contaminated sediment $(\mathrm{Tc}-2)$ samples. Initial sulfate concentrations were varied. As +5 was used because its ionic character is more like sulfate than As +3 (Ferguson and Gavis, 1972). Results are listed in table 15.

Contaminated sediment samples were used in duplicate batch experiments to determine the variability of adsorption among samples and sample splits. Final arsenic and sulfate concentration are analyzed for. Results are listed in table 16.

Partical-size splits of both the uncontaminated sediment $(A C-1)$ and contaminated sediment ( $\mathrm{Tc}-2$ ) were obtained using polypropylene sieves. Batch experiments using both $\mathrm{As}+3$ and $\mathrm{As}+5$ initial concentrations were run to determine the effect of grain size on adsorption. Results are listed in table 17. 


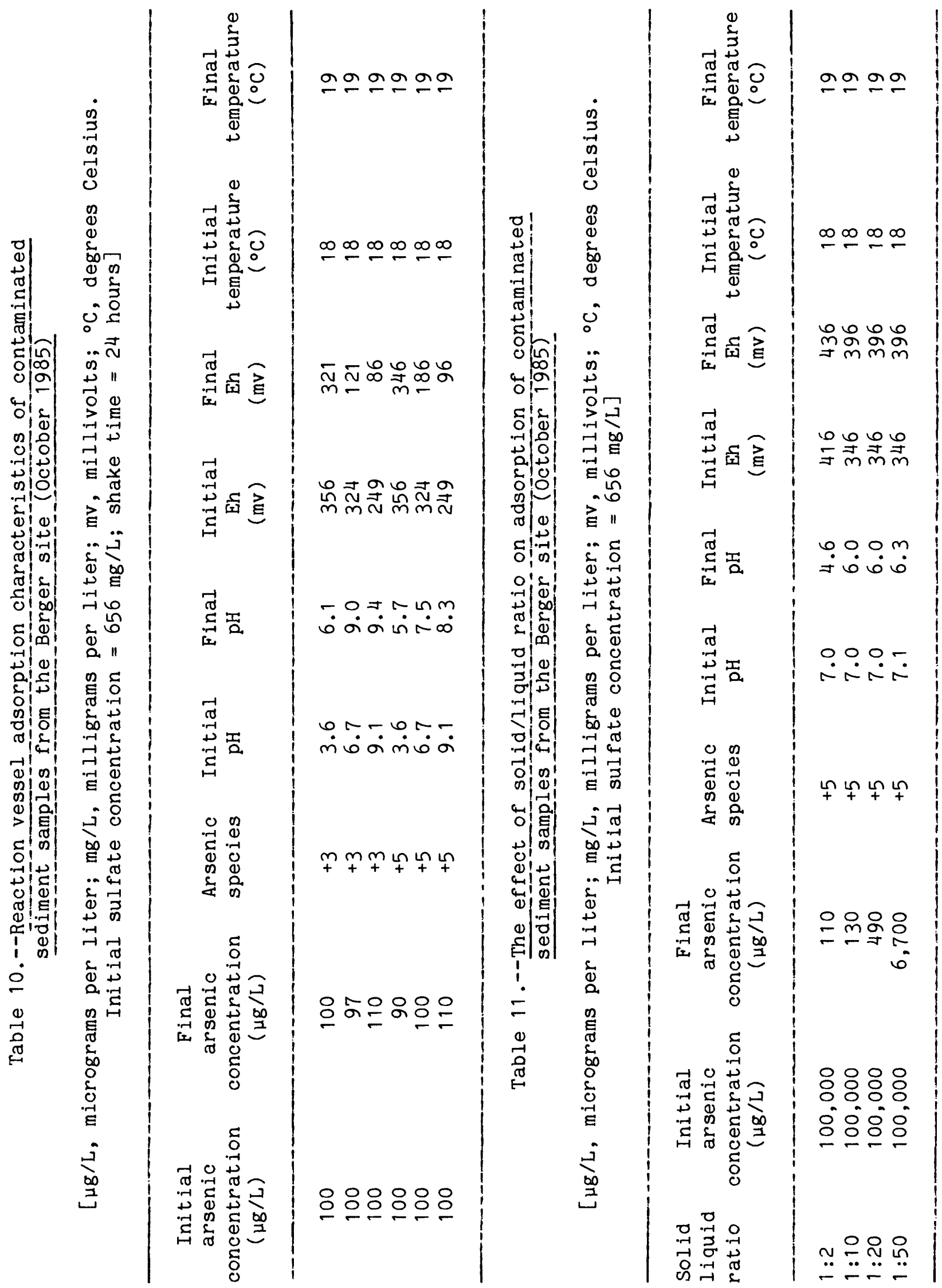




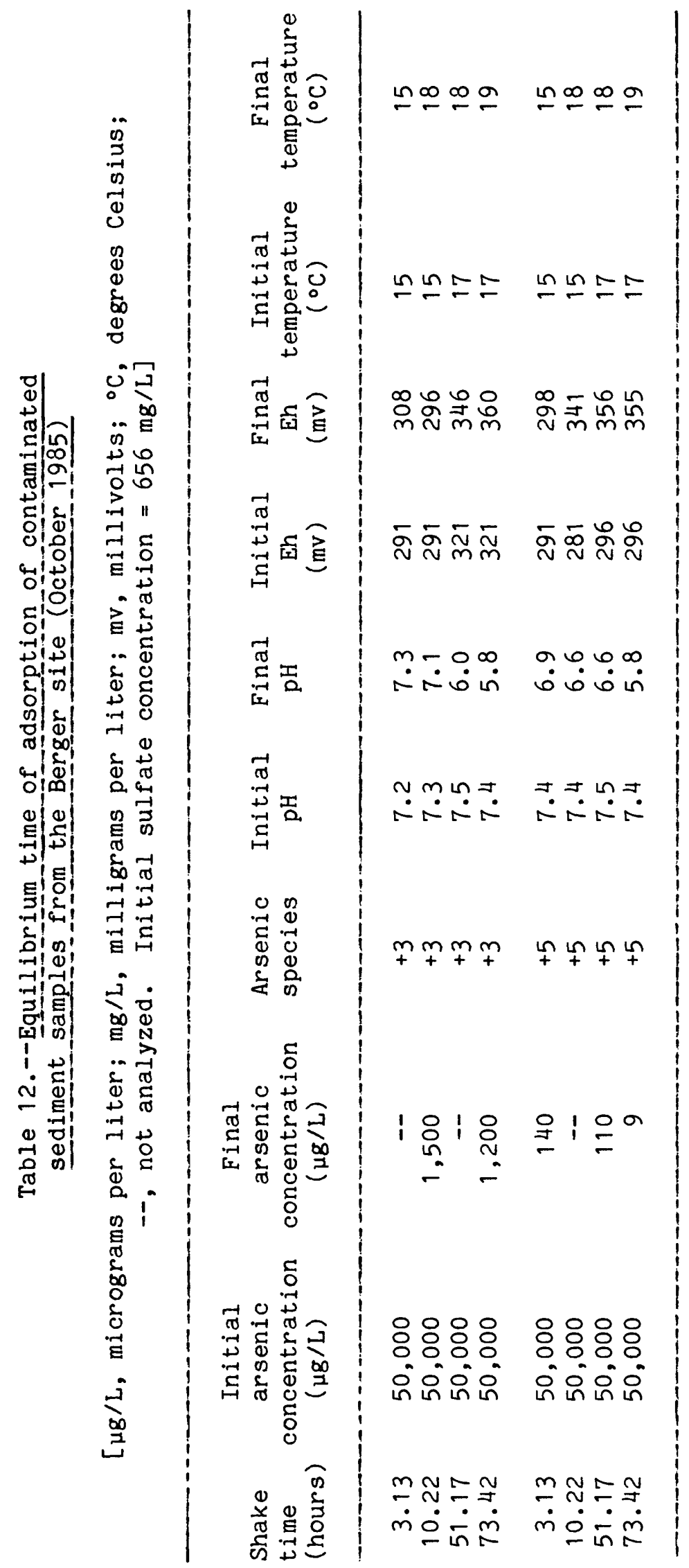




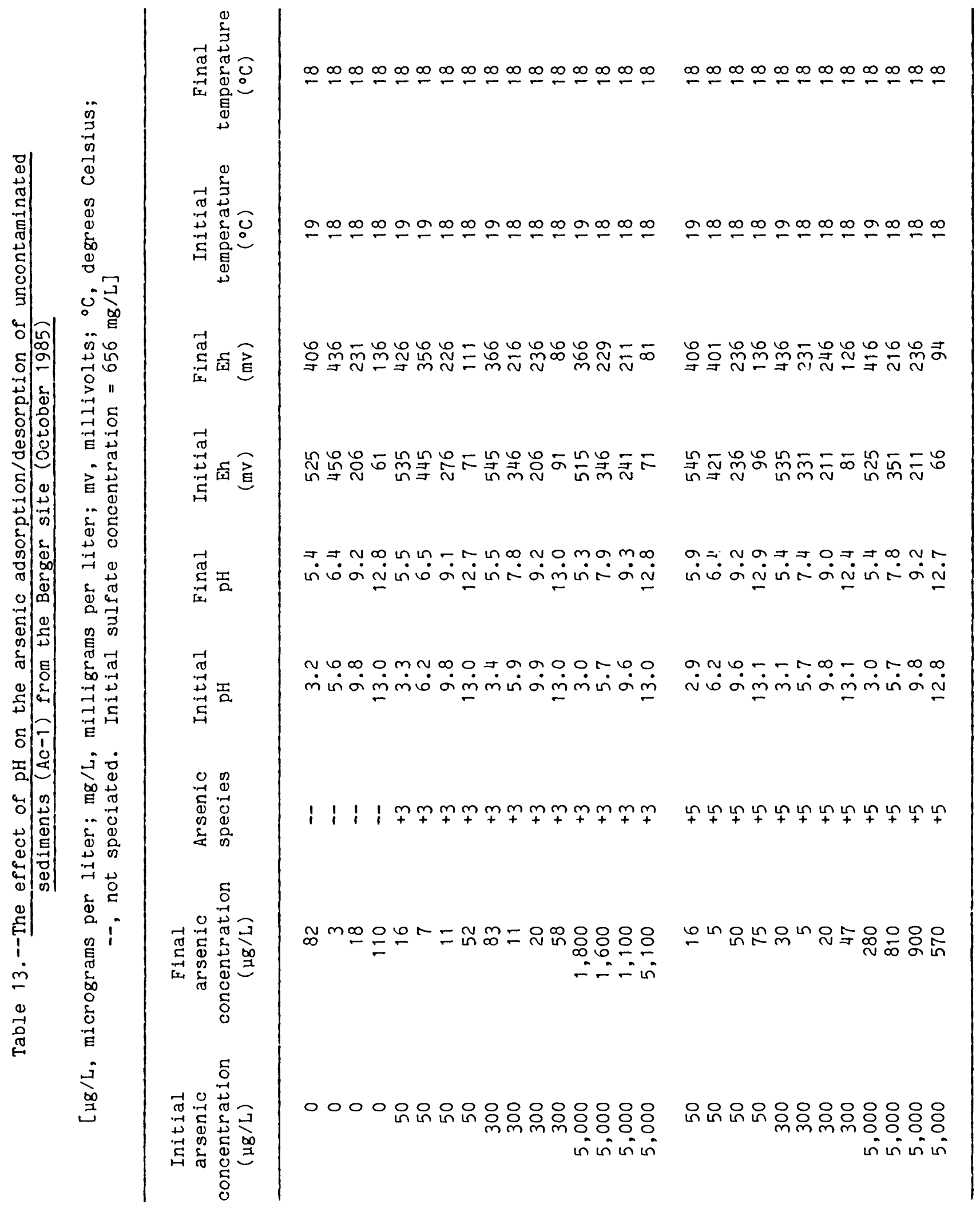




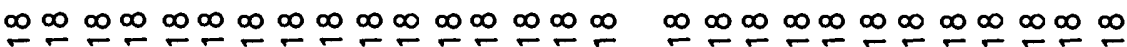

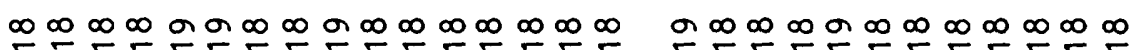

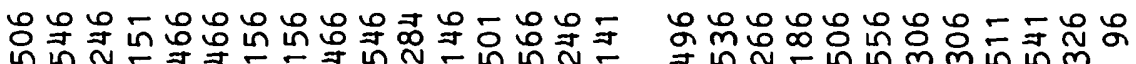

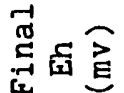

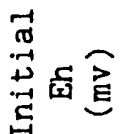

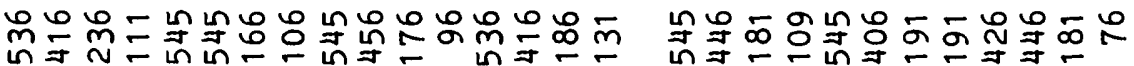

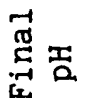

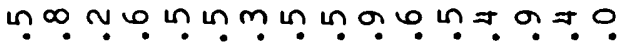

ln 00 Trt.

$\dot{m} \dot{x} \infty=\dot{m} \dot{m}=\dot{m} \dot{=}=\dot{m} \dot{a} \dot{i}$

$\dot{m}=\dot{\sigma}=\dot{m} \dot{\sigma} \dot{\infty} \dot{m} \dot{m} \dot{\infty}$

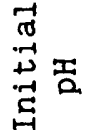

a

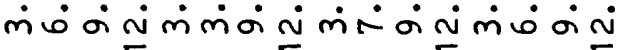

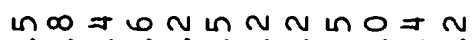

$\dot{m} \dot{0} \ddot{\sim} \dot{m} \dot{\sigma} \dot{\sigma} \dot{m} \dot{\sigma} \dot{m}$

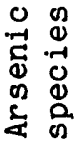

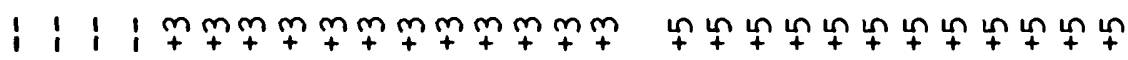

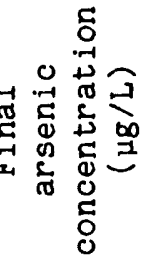

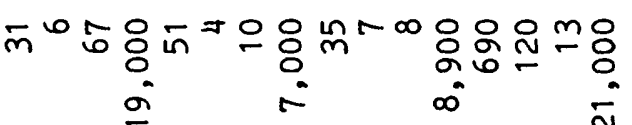

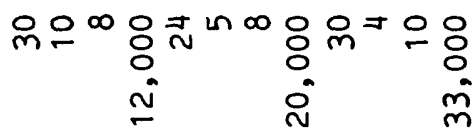

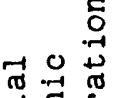

iิ

至

点 。

-

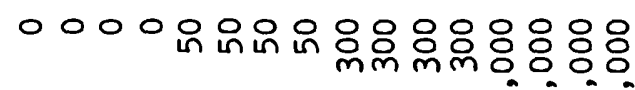

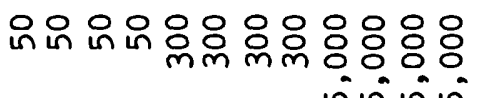



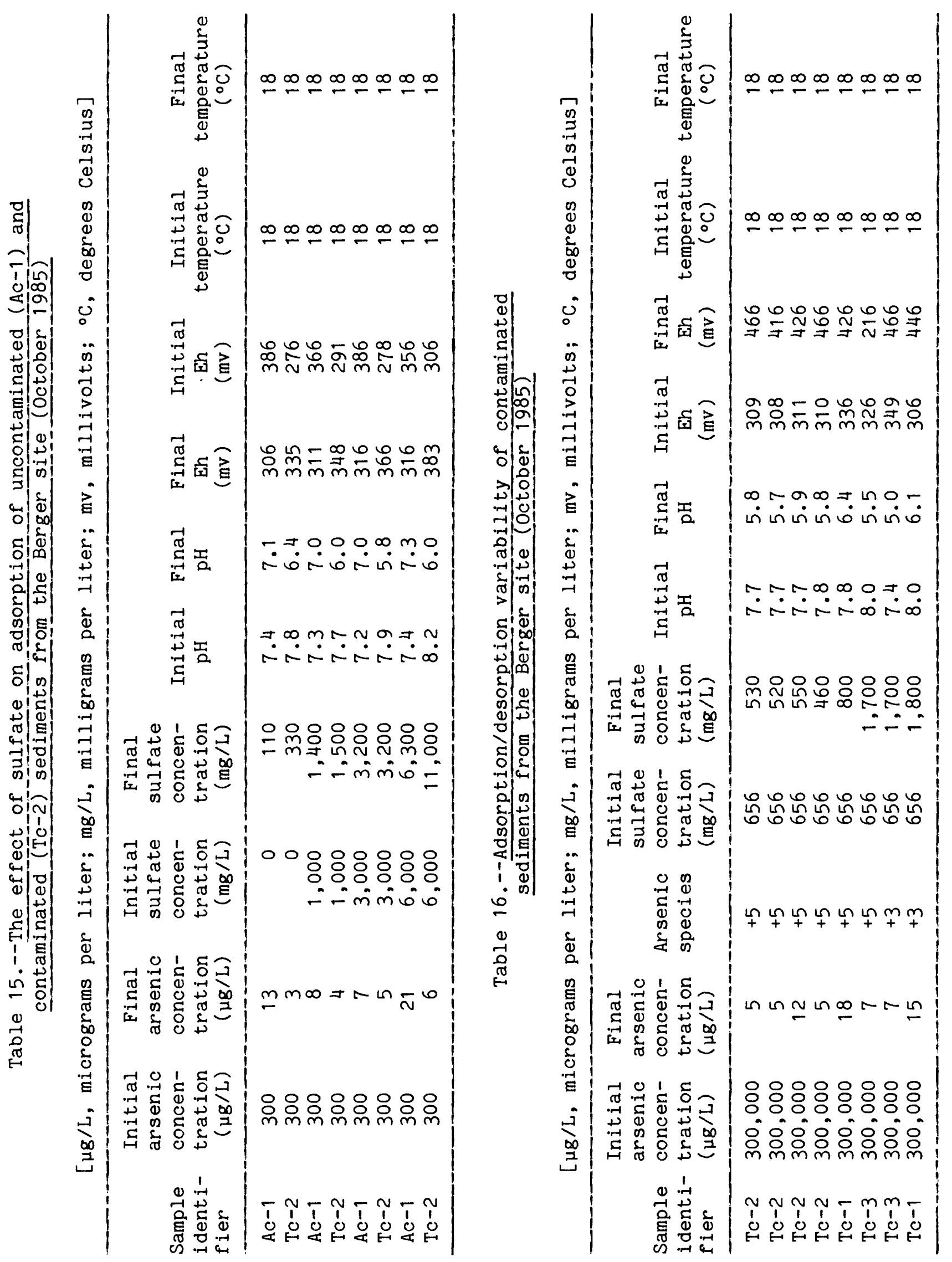


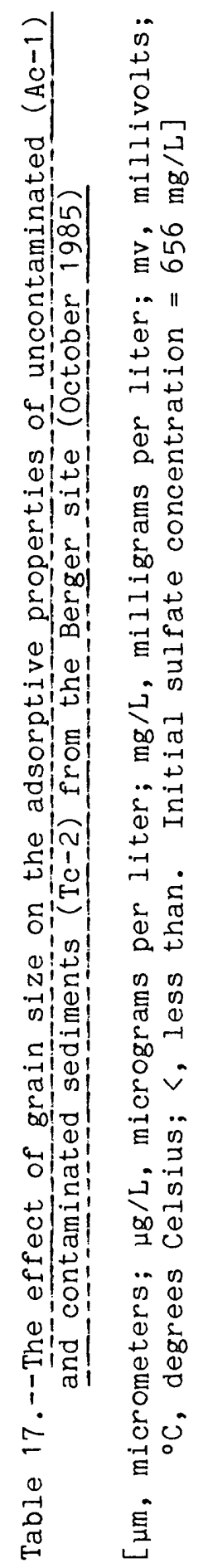

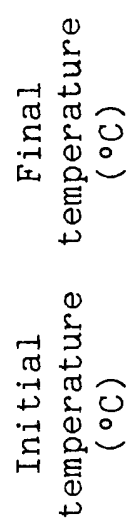

드느느느는

ตำ

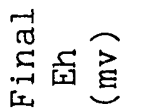

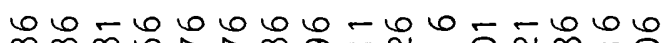

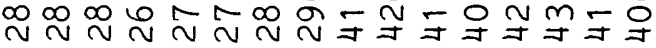

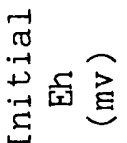

テ

เ

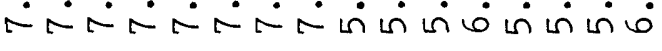

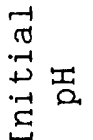

$0.0 \div-\div 0.0 \mathrm{~mm}$.

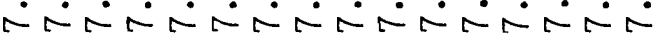

00

$\begin{array}{cc}0 & 0 \\ -1 & 0 \\ 1 & -1 \\ 0 & 0 \\ 0 & 0 \\ 5 & 0 \\ \text { \& } & 0\end{array}$

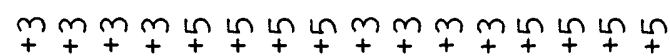

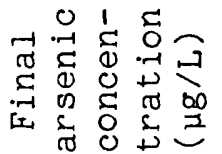

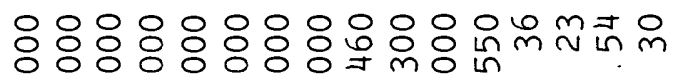
îm

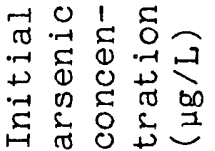

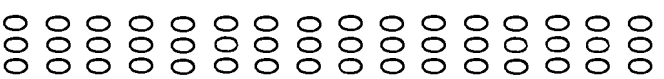

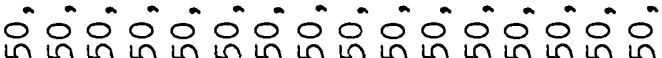

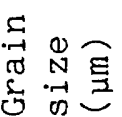

응응 $\mathrm{m}$ 응 $\mathrm{mm}$ 응 응 $\mathrm{m}$ 응 $\mathrm{mm}$

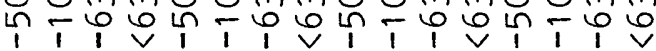

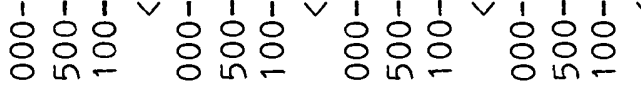

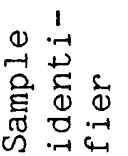

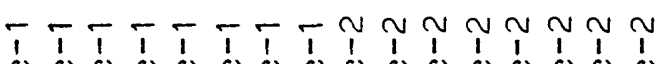

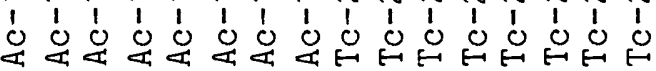


Foot-long columns made from PVC pipe were rinsed thoroughly and soaked overnight in distilled water. Uncontaminated $(\mathrm{Ac}-1)$ and contaminated $(\mathrm{TC}-2)$ freeze-dried sediment was sieved with a 1-mm polypropylene screen and hand packed in the columns using increments of $10 \mathrm{~g}$ of sediment. Resultant permeability approximated field values. Column tops and bottoms were packed with 0.25 inch of glass wool.

Columns were first saturated for 7 days under constant head by water with varied sulfate concentrations, and the leachate was sampled for arsenic. Then water containing varied arsenic and sulfate concentrations was fed to the columns under constant head. Periodically, lab parameters were measured and leachate was sampled for arsenic analysis. Sediment porosities were measured after completion of the experiment. Column hydraulic characteristics are listed in table 18. Results of column experiments are listed in tables 19 and 20 .

Table 18.--Hydraulic characteristics, in columns, of uncontaminated (Ac-1) and contaminated (Tc-2) sediments from the Berger site (October 1985)

$\left[\mathrm{ft}^{3} / \mathrm{s}\right.$, cubic feet per second; $\mathrm{ft} / \mathrm{s}$, feet per second]

\begin{tabular}{|c|c|c|c|c|}
\hline Column & Deposit & $\begin{array}{l}\text { Pore volume } \\
\left(\mathrm{ft}^{3} / \mathrm{s}\right)\end{array}$ & $\begin{array}{l}\text { Porosity } \\
(\%)\end{array}$ & $\begin{array}{c}\text { Hydraulic conductivity } \\
(\mathrm{ft} / \mathrm{s})\end{array}$ \\
\hline
\end{tabular}

\begin{tabular}{lllll}
1 & $\mathrm{Ac}-1$ & $4.802 \times 10^{-3}$ & 0.50 & $9.51 \times 10^{-7}$ \\
2 & $\mathrm{Ac}-1$ & $4.346 \times 10^{-3}$ & .44 & $2.79 \times 10^{-7}$ \\
3 & $\mathrm{Ac}-1$ & $4.717 \times 10^{-3}$ & .48 & $3.346 \times 10^{-6}$ \\
4 & $\mathrm{Ac}-1$ & $4.799 \times 10^{-3}$ & .48 & $8.53 \times 10^{-6}$ \\
6 & $\mathrm{Tc}-2$ & $6.073 \times 10^{-3}$ & .62 & $1.94 \times 10^{-5}$ \\
7 & $\mathrm{Tc}-2$ & $5.897 \times 10^{-3}$ & .58 & $8.86 \times 10^{-6}$ \\
8 & $\mathrm{Tc}-2$ & $5.720 \times 10^{-3}$ & .57 & $1.48 \times 10^{-5}$ \\
\hline
\end{tabular}




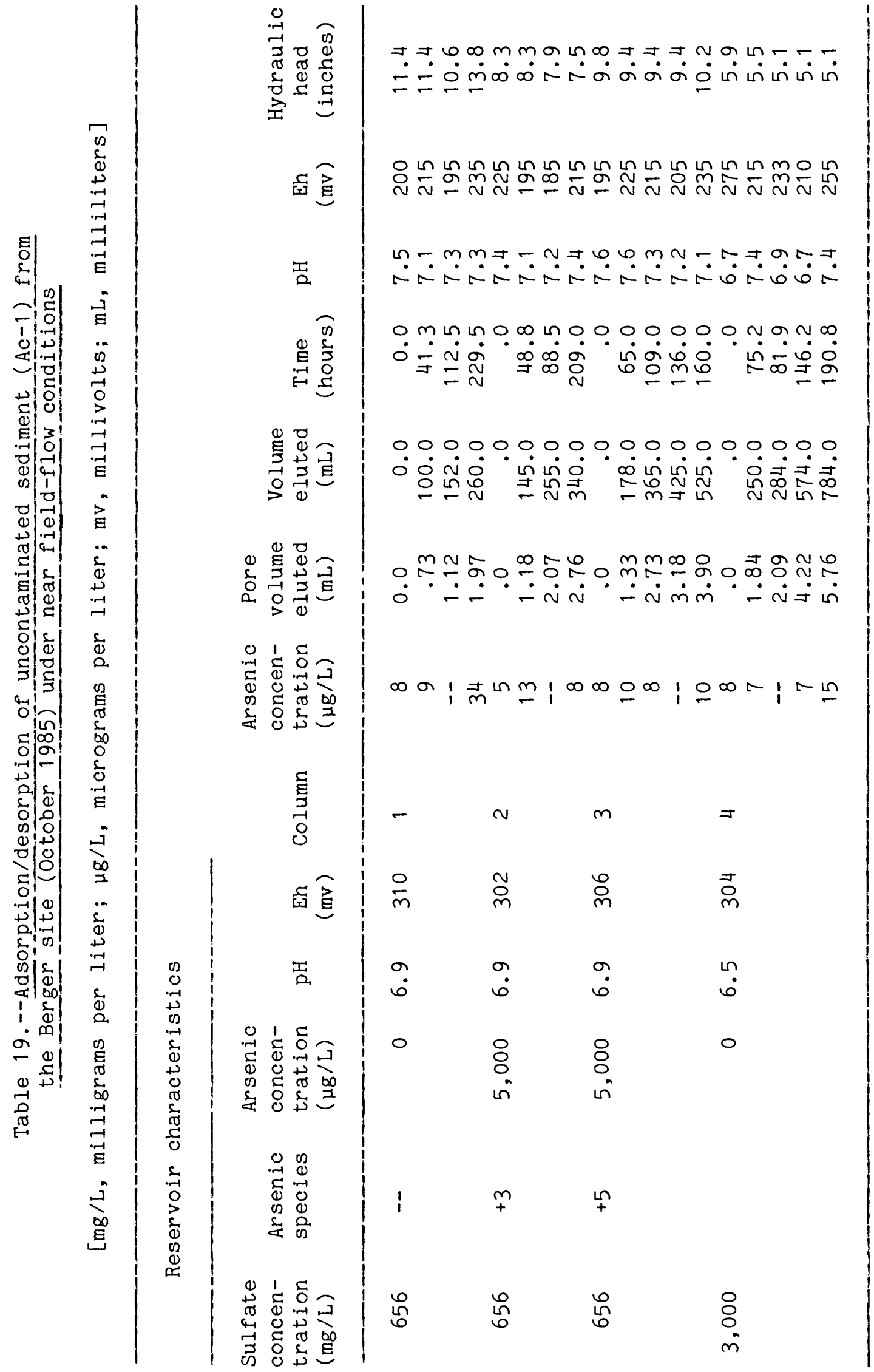




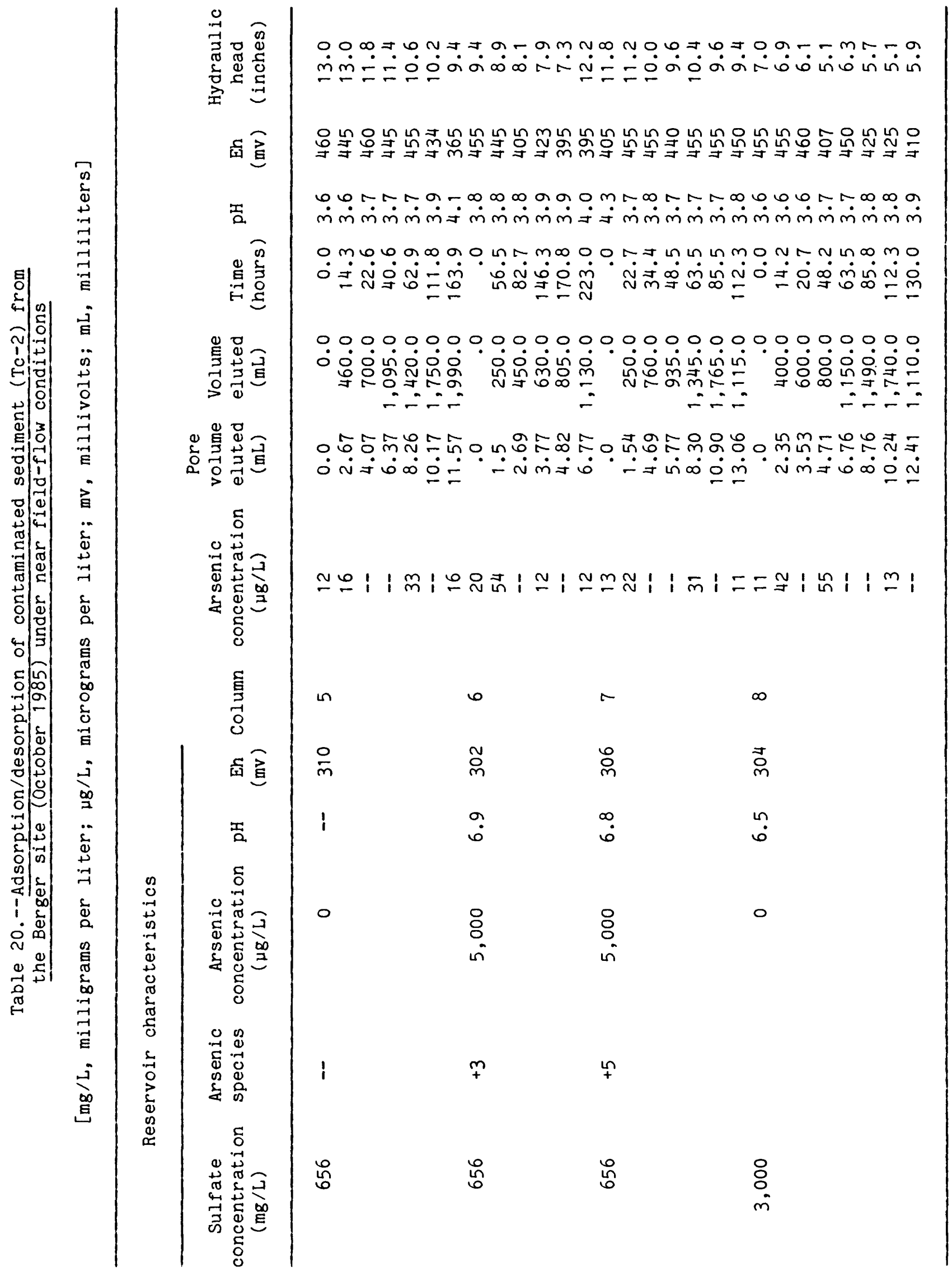




\section{REFERENCES}

Bodman, G.B., 1928, The hydrogen peroxide-hydrochloric acid treatment of soils as a method of dispersion in mechanic analysis: Soil Science, v. 26, p. 459-470.

Cherry, J.A., Morel, F.M.M., Rouse, J.V., Schnoor, J.L., and Wolman, M.G., 1984, Hydrogeo-chemistry of sulfide and arsenic-rich tailings and alluvium along Whitewood Creek, South Dakota: Mineral and Energy Resources Series, Colorado School of Mines.

Feltz, H.R., and Anthony, E.R., eds., 1985, Water quality laboratory services catalog: U.S. Geological Survey Open-File Report 84-171.

Ferguson, J.F., and Gavis, J., 1972, A review of the arsenic cycle in natural waters: Water Research, v. 6, p. 1259-1274.

Fetter, C.W., Jr., 1980, Applied hydrogeology: Charles E. Merrill Publishing Company, Columbus, Ohio, $488 \mathrm{p}$.

Fox Associates, 1984, Technical assessment of environmental issues in the Whitewood Creek area: Unpublished report for Homestake Mining Company, $8 \mathrm{p}$.

Friedman, G.M., and Johnson, K.G., 1982, Exercises in sedimentology: John Wiley and Sons, New York, $208 \mathrm{p}$.

Frost, R.R., and Griffin, R.A., 1977, Effect of $p H$ on adsorption of arsenic and selenium from landfill leachate by clay minerals: Soil Science Society American Journal, v. 41, p. 53-57.

Goddard, K.E., in press, Composition, distribution, and hydrologic effects of contaminated sediments resulting from the discharge of gold-mill tailings to Whitewood Creek at Lead and Deadwood, South Dakota: U.S. Geological Survey Water-Resources Investigations Report 87-4051.

Olson, S.R., and Watanabe, F.S., 1957, A method to determine a phosphorus adsorption maximum of soils as measured by the Langmuir Isotherm: Soil Science Society Proceedings, p. 144-149.

Stach, R.L., Helgerson, R.N., Bretz, R.F., Tipton, M.J., Beissel, D.R., and Harksen, J.C., 1978, Arsenic levels in the surface and ground waters along Whitewood Creek, Belle Fourche River, and a portion of the Cheyenne River, South Dakota: Water Resources Institute/South Dakota Geological Survey completion report, $41 \mathrm{p}$.

Wuolo, R.W., 1986, Laboratory studies of arsenic adsorption in alluvium contaminated with gold-mine tailings along Whitewood Creek, Black Hills, South Dakota: Master's Thesis, South Dakota School of Mines and Technology, $166 \mathrm{p}$. 


\section{Chapter B. Trace-Element Geochemistry of Sediments Collected from the Flood Plains of Whitewood Creek and the Belle Fourche and Cheyenne Rivers}

By Donna C. Marron

\section{SAMPLE COLLECTION AND HANDLING PROCEDURES}

Samples of flood-plain sediment were collected for chemical analysis during the summers of 1985 and 1986. Samples were collected from soil pits that were dug to a depth of $1 \mathrm{~m}$ along the flood plains of Whitewood Creek, the Belle Fourche River, and the Cheyenne River. The soil pits were arranged along transects that were perpendicular to the modern stream channel. Transect locations are shown in figures 1 and 2 and listed in table 1.

The stratigraphy that was observed in each soil pit was measured and described. A variety of layers was sampled. Plastic containers and sampling utensils were used. The containers and sampling utensils were soaked in 5 percent HCL, rinsed with deionized water, and air dried before use. Samples were frozen at the end of the day they were collected. Distilled water was added to the frozen samples to make a slurry immediately before freeze drying. The slurry was then frozen onto the insides of freeze-drying flasks and freeze dried. The freeze-drying flasks were soaked in 5 percent HCL and rinsed with deionized water before use.

Splits of samples were made using a plastic or a stainless steel splitter. Splits of samples were collected in glass or ceramic dishes. Utensils used for splitting samples were rinsed in 5 percent HCL and deionized water, and then were air dried before use. Splits of four samples were separated into grain-size fractions using an air elutriator.

\section{ANALYTICAL PROCEDURES AND RESULTS}

Several types of digestion procedures were used. "Partial" digestion analyses for arsenic used a nitric and sulfuric acid digestion (Method I-606285 in Fishman and Friedman, 1985) and atomic absorption spectrometry. "Partial" digestion analyses for aluminum, antimony, chromium, copper, manganese, and zinc used a weak-acid digestion (Method I-5485-85 in Fishman and Friedman, 1985) and atomic absorption spectrometry. "Partial" digestion analyses for mercury used method I-5462-85 of Fishman and Friedman, 1985. "Total" digestion analyses were done using total digestion of the sample with $\mathrm{HF} / \mathrm{HClO}_{4} / \mathrm{HNO}_{3}$ acid with detection by inductively coupled plasma atomic emission spectrometer. Analytical results are listed in tables 21,22 , and 23. Selected samples were analyzed for grain-size distributions and the results are listed in table 24 .

\section{REFERENCES}

Fishman, M.J., and Friedman, L.C., eds., 1985, Methods of determination of inorganic substances in water and fluvial sediments: U.S. Geological Survey Techniques of Water-Resources Investigations, book 5, chap. A1, $709 \mathrm{p}$. 
Table 22.--Total digestion analysis of sediment samples collected during 1985 and 1986 from the flood plains of Whitewood Creek and the Belle Fourche and Cheyenne Rivers

$[\mu \mathrm{g} / \mathrm{g}$, micrograms per gram $]$

\begin{tabular}{|c|c|c|c|c|c|c|c|c|c|}
\hline $\begin{array}{c}\text { Sample } \\
\text { identifier }\end{array}$ & $\begin{array}{l}\text { Aluminum } \\
(\not)\end{array}$ & $\begin{array}{l}\text { Calcium } \\
(\text { (p) }\end{array}$ & $\begin{array}{l}\text { Iron } \\
(p)\end{array}$ & $\begin{array}{c}\text { Potassium } \\
(\text { (q) }\end{array}$ & $\begin{array}{l}\text { Magnesium } \\
(\%)\end{array}$ & $\begin{array}{l}\text { Sodium } \\
(\not)\end{array}$ & $\begin{array}{c}\text { Phosphorous } \\
\text { ( })\end{array}$ & $\begin{array}{l}\text { Titanium } \\
(\not)\end{array}$ & $\begin{array}{l}\text { Arsenic } \\
(\mu \mathrm{g} / \mathrm{g})\end{array}$ \\
\hline WW $1 \mathrm{~A} 1$ & 4.1 & 1.1 & 11 & 1.6 & 1.4 & 0.31 & 0.07 & 0.19 & 1,400 \\
\hline WW1A2 & 1.6 & .14 & 17 & .63 & .89 & .15 & .07 & .12 & 2,100 \\
\hline WW1A3 & 3.4 & 2.0 & 13 & .80 & 1.7 & .19 & .05 & .17 & 1,300 \\
\hline WW1Bi & 1.6 & .06 & 17 & .65 & .90 & .14 & .07 & .11 & 1,900 \\
\hline WW1B2 & 3.0 & .55 & 14 & 1.2 & .74 & .24 & .07 & .17 & 980 \\
\hline WW1B2 & 3.1 & .76 & 13 & 1.2 & .78 & .23 & .06 & .16 & 970 \\
\hline$W W 1 C^{1}$ & 1.8 & .62 & 13 & .72 & .95 & .15 & .06 & .11 & 1,900 \\
\hline $\mathrm{WW} 1 \mathrm{Cl}^{1}$ & 2.0 & .66 & 14 & .88 & 1.1 & .16 & .06 & .10 & 1,700 \\
\hline WW1C2 & 2.3 & 1.1 & 9.4 & .69 & 1.3 & .16 & .03 & .14 & 760 \\
\hline WW2A1 & 2.9 & 2.5 & 11 & .98 & 1.1 & .22 & .05 & .14 & 3,400 \\
\hline WW2A2 & 2.8 & 1.8 & 13 & .81 & 1.3 & .19 & .05 & .14 & 4,100 \\
\hline WW2A 3 & 2.5 & 1.5 & 15 & .65 & 1.4 & .17 & .05 & .13 & 6,500 \\
\hline WW2A 4 & 3.2 & 1.3 & 15 & .91 & 1.7 & .18 & .05 & .14 & 4,600 \\
\hline WW2B1 & 2.6 & .34 & 14 & 1.1 & 1.0 & .22 & .05 & .13 & 3,800 \\
\hline WW2B2 & 2.3 & .74 & 11 & .96 & .88 & .17 & .06 & .10 & 1,500 \\
\hline WW2B3 & 3.7 & .76 & 14 & 1.1 & 1.2 & .19 & .08 & .17 & 1,400 \\
\hline WW2B4 & 5.1 & 2.6 & 2.5 & 1.4 & .37 & .39 & .08 & .17 & 40 \\
\hline $\mathrm{BF}|\mathrm{A}|$ & 4.2 & 1.7 & 4.4 & 1.5 & .83 & .35 & .06 & .19 & 320 \\
\hline $\mathrm{BF} 1 \mathrm{~A} 2$ & 2.8 & 1.5 & 5.8 & 1.1 & .62 & .22 & .05 & .12 & 590 \\
\hline$B F 1 A 3$ & 2.6 & 1.2 & 11 & 1.2 & .86 & .21 & .04 & .11 & 1,100 \\
\hline $\mathrm{BF} 1 \mathrm{~B} 2$ & 4.5 & 2.4 & 12 & 1.4 & 2.0 & .29 & .07 & .20 & 2,100 \\
\hline $\mathrm{BF} 1 \mathrm{~B} 3$ & 2.4 & 1.3 & 10 & 1.1 & .97 & .21 & .04 & .09 & 1,400 \\
\hline $\mathrm{BF} 1 \mathrm{C} 1^{1}$ & 3.0 & 1.4 & 16 & .73 & 2.1 & .22 & .05 & .16 & 3,100 \\
\hline $\mathrm{BF} 1 \mathrm{C} 1^{1}$ & 3.3 & 1.2 & 16 & .83 & 2.2 & .23 & .05 & .15 & 2,700 \\
\hline $\mathrm{BF} 1 \mathrm{C} 2$ & 5.1 & 2.1 & 12 & 1.4 & 2.5 & .27 & .07 & .21 & 2,300 \\
\hline $\mathrm{BF} 2 \mathrm{~A} 1$ & 3.1 & .27 & 12 & 1.1 & 1.2 & .30 & .05 & .15 & 2,000 \\
\hline $\mathrm{BF} 2 \mathrm{~A} 2$ & 4.6 & 1.4 & 12 & 1.3 & 2.2 & .26 & .06 & .19 & 720 \\
\hline $\mathrm{BF} 2 \mathrm{~B} 1$ & 2.7 & 1.1 & 12 & .94 & 1.4 & .27 & .04 & .13 & 1,600 \\
\hline $\mathrm{BF} 2 \mathrm{~B} 2$ & 3.3 & 1.4 & 11 & 1.1 & 1.4 & .24 & .06 & .15 & 670 \\
\hline $\mathrm{BF} 2 \mathrm{C} 1$ & 4.3 & 1.0 & 11 & 1.2 & 1.8 & .34 & .06 & .19 & 1,700 \\
\hline $\mathrm{BF} 2 \mathrm{C} 2$ & 2.6 & 1.4 & 14 & .72 & 1.2 & .21 & .04 & .13 & 2,200 \\
\hline $\mathrm{BF} 2 \mathrm{C} 3$ & 2.6 & 1.5 & 11 & .61 & 1.4 & .20 & .04 & .12 & 1,400 \\
\hline $\mathrm{BF} 2 \mathrm{D} 2$ & 5.1 & 1.5 & 5.9 & 1.6 & .90 & .49 & .06 & .22 & 450 \\
\hline $\mathrm{BF} 2 \mathrm{D} 3$ & 2.9 & .87 & 9.7 & 1.1 & .93 & .26 & .06 & .15 & 770 \\
\hline $\mathrm{BF} 2 \mathrm{E}$ & 5.6 & 1.5 & 2.9 & 1.7 & .85 & .49 & .06 & .25 & 10 \\
\hline $\mathrm{BF} 2 \mathrm{~N} 1$ & 5.4 & 1.6 & 2.7 & 1.7 & .96 & .51 & .06 & .25 & 20 \\
\hline $\mathrm{BF} 2 \mathrm{~N} 2^{1}$ & 3.7 & 4.4 & 1.7 & 1.5 & .88 & .45 & .06 & .16 & $<10$ \\
\hline $\mathrm{BF} 2 \mathrm{~N} 2^{1}$ & 4.0 & 4.6 & 1.9 & 1.9 & .96 & .45 & .06 & .16 & $<10$ \\
\hline BF $3 A 1$ & 2.3 & 2.0 & 16 & .65 & 1.3 & .30 & .04 & .14 & 5,000 \\
\hline $\mathrm{BF} 3 \mathrm{~A} 2$ & 7.6 & 1.3 & 4.8 & 1.7 & 1.4 & .54 & .05 & .31 & 260 \\
\hline $\mathrm{BF} 3 \mathrm{B1}$ & 3.0 & 1.3 & 14 & .75 & 1.5 & .25 & .04 & .14 & 1,700 \\
\hline $\mathrm{BF} 3 \mathrm{~B}^{1}$ & 3.8 & 1.4 & 13 & .87 & 2.0 & .22 & .06 & .18 & 2,800 \\
\hline $\mathrm{BF} 3 \mathrm{~B} 2^{1}$ & 4.0 & 1.2 & 13 & .96 & 2.1 & .23 & .06 & .17 & 2,400 \\
\hline $\mathrm{BF}_{4} \mathrm{~A} 1$ & 4.0 & 1.1 & 11 & 1.1 & 1.6 & .33 & .06 & .17 & 1,800 \\
\hline $\mathrm{BF}_{4} \mathrm{~A} 3$ & 5.8 & 1.6 & 6.9 & 1.5 & 1.3 & .43 & .06 & .24 & 570 \\
\hline $\mathrm{BF} 4 \mathrm{~B} 3$ & 3.6 & .75 & 7.4 & 1.2 & .91 & .31 & .05 & .16 & 710 \\
\hline $\mathrm{BF} 4 \mathrm{~B} 4$ & 3.5 & 1.4 & 6.4 & 1.2 & .91 & .29 & .05 & .14 & 410 \\
\hline $\mathrm{BF} 4 \mathrm{R} 1^{1}$ & 3.8 & 1.7 & 12 & .82 & 2.0 & .25 & .05 & .17 & 1,700 \\
\hline $\mathrm{BF} 4 \mathrm{R} 1^{1}$ & 4.1 & 1.7 & 13 & .93 & 2.1 & .25 & .06 & .17 & 1,800 \\
\hline $\mathrm{BF} 4 \mathrm{R} 2$ & 3.6 & 1.3 & 10 & .91 & 1.7 & .30 & .05 & .16 & 1,400 \\
\hline BF5A 1 & 5.2 & .86 & 7.8 & 1.5 & 1.3 & .43 & .07 & .23 & 850 \\
\hline $\mathrm{BF} 5 \mathrm{~B} 1$ & 4.3 & .99 & 11 & 1.1 & 1.7 & .35 & .06 & .19 & 1,300 \\
\hline BF5B2 & 4.9 & .86 & 11 & 1.1 & 2.1 & .36 & .05 & .21 & 1,200 \\
\hline BF5B3 & 3.3 & 1.6 & 12 & .86 & 1.6 & .31 & .06 & .16 & 1,900 \\
\hline $\mathrm{BF} 5 \mathrm{~B} 4$ & 6.4 & 1.0 & 6.2 & 1.7 & 1.4 & .54 & .07 & .26 & 490 \\
\hline $\mathrm{BF} 5 \mathrm{C} 1$ & 5.6 & 1.6 & 7.1 & 1.5 & 1.4 & .41 & .07 & .24 & 650 \\
\hline $\mathrm{BF} 5 \mathrm{C} 2$ & 4.1 & 1.3 & 12 & .99 & 1.4 & .35 & .06 & .18 & 1,700 \\
\hline $\mathrm{BF} 5 \mathrm{C} 3$ & 4.0 & 1.1 & 11 & .96 & 1.9 & .36 & .06 & .18 & 1,300 \\
\hline CR $|A|$ & 5.5 & 3.1 & 3.7 & 1.8 & .91 & 1.0 & .07 & .20 & 200 \\
\hline$C R 1 A 2^{1}$ & 5.5 & 2.2 & 5.8 & 1.7 & 1.3 & .85 & .07 & .22 & 410 \\
\hline CR1A2 & 5.6 & 2.1 & 6.0 & 1.7 & 1.3 & .81 & .07 & .22 & 430 \\
\hline$C R 1 A 3$ & 8.4 & 1.7 & 5.5 & 2.1 & 1.5 & .57 & .08 & .33 & 260 \\
\hline CR $1 A^{4}$ & 5.1 & 2.2 & 7.8 & 1.5 & 1.6 & .76 & .07 & .22 & 830 \\
\hline CR2B1 & 4.4 & 2.1 & 3.4 & 1.6 & .72 & .77 & .06 & .17 & 170 \\
\hline CR2B2 & 4.3 & 1.9 & 3.6 & 1.6 & .70 & .82 & .06 & .18 & 180 \\
\hline
\end{tabular}


Table 22.--Total digestion analysis of sediment samples collected during 1985 and 1986 from the flood plains of Whitewood Creek and the Belle Fourche and Cheyenne Rivers--Continued

\begin{tabular}{|c|c|c|c|c|c|c|c|c|c|}
\hline $\begin{array}{c}\text { Sample } \\
\text { Identifler }\end{array}$ & $\begin{array}{l}\text { Barium } \\
(\mu g / g)\end{array}$ & $\begin{array}{l}\text { Cer Ium } \\
(\mu g / g)\end{array}$ & $\begin{array}{c}\text { Chromlum } \\
(\mu \mathrm{g} / \mathrm{g})\end{array}$ & $\begin{array}{l}\text { Cobalt } \\
(\mu g / 8)\end{array}$ & $\begin{array}{l}\text { Copper } \\
(\mu \mathrm{g} / \mathrm{g})\end{array}$ & $\begin{array}{l}\text { Gall1um } \\
(\mu \mathrm{g} / \mathrm{g})\end{array}$ & $\begin{array}{l}\text { Lanthanum } \\
(\mu \mathrm{g} / \mathrm{g})\end{array}$ & $\begin{array}{l}\text { Lithium } \\
(\mu \mathrm{g} / \mathrm{g})\end{array}$ & $\begin{array}{c}\text { Manganese } \\
(\mu \mathrm{g} / \mathrm{g})\end{array}$ \\
\hline WW1A1 & 420 & 51 & 75 & 20 & 78 & 13 & 28 & 28 & 2,500 \\
\hline WW1A2 & 240 & 59 & 36 & 10 & 57 & 7 & 33 & 10 & 1,700 \\
\hline WW1A3 & 280 & 35 & 56 & 8 & 30 & 13 & 19 & 18 & 1,200 \\
\hline WW1B1 & 200 & 36 & 34 & 12 & 65 & 5 & 21 & 10 & 1,200 \\
\hline WW1B2 & 320 & 49 & 58 & 16 & 97 & 10 & 27 & 19 & 960 \\
\hline WW1B2 & 300 & 36 & 47 & 16 & 79 & 11 & 20 & 19 & 960 \\
\hline $\mathrm{WW} 1 \mathrm{Cl}^{\prime}$ & 220 & 28 & 25 & 8 & 38 & 6 & 17 & 11 & 1,100 \\
\hline WWICl' & 250 & 34 & 29 & 7 & 48 & 7 & 21 & 13 & 1,200 \\
\hline WW1C2 & 250 & 29 & 30 & 6 & 20 & 8 & 17 & 13 & 1,100 \\
\hline WW $2 A 1$ & 300 & 36 & 35 & 17 & 57 & 10 & 20 & 18 & 2,000 \\
\hline WW2A2 & 270 & 39 & 44 & 22 & 73 & 12 & 22 & 16 & 3,800 \\
\hline WW $2 A 3$ & 210 & 31 & 32 & 14 & 92 & 10 & 19 & 13 & 2,300 \\
\hline WW2A4 & 260 & 36 & 51 & 10 & 120 & 12 & 19 & 17 & 1,800 \\
\hline WW2B1 & 280 & 28 & 33 & 8 & 44 & 10 & 17 & 13 & 1,400 \\
\hline WW2B2 & 250 & 45 & 37 & 12 & 55 & 8 & 25 & 15 & 1,400 \\
\hline WW2B3 & 320 & 29 & 58 & 22 & 91 & 14 & 17 & 21 & 3,400 \\
\hline WW2B4 & 340 & 57 & 52 & 61 & 190 & 12 & 30 & 52 & 4,200 \\
\hline $\mathrm{BE} 1 \mathrm{~A} 1$ & 570 & 48 & 50 & 11 & 31 & 10 & 27 & 26 & 1,000 \\
\hline$B F 1 A 2$ & 440 & 37 & 28 & 11 & 31 & 8 & 21 & 16 & 1,400 \\
\hline$B F 1 A 3$ & 310 & 39 & 28 & 12 & 53 & 8 & 22 & 15 & 1,500 \\
\hline BE 1B2 & 400 & 50 & 73 & 19 & 89 & 14 & 28 & 30 & 2,900 \\
\hline $\mathrm{BF} 1 \mathrm{~B} 31$ & 290 & 23 & 25 & 7 & 20 & 8 & 15 & 10 & 1,400 \\
\hline $\mathrm{BF} 1 \mathrm{C} 1^{\prime}$ & 230 & 26 & 42 & 18 & 80 & 13 & 16 & 16 & 3,300 \\
\hline $\mathrm{BF} 1 \mathrm{Cl}^{1}$ & 250 & 33 & 49 & 15 & 87 & 12 & 20 & 18 & 3,100 \\
\hline $\mathrm{BF} 1 \mathrm{C} 2$ & 390 & 52 & 81 & 16 & 99 & 17 & 29 & 35 & 3,300 \\
\hline BF $2 A 1$ & 400 & 32 & 46 & 8 & 50 & 9 & 19 & 15 & 1,600 \\
\hline$B F 2 A 2$ & 380 & 40 & 65 & 14 & 60 & 15 & 22 & 35 & 3,000 \\
\hline BF 2B1 & 290 & 35 & 40 & 7 & 43 & 9 & 20 & 15 & 1,800 \\
\hline BF 2B2 & 340 & 39 & 46 & 14 & 51 & 11 & 22 & 24 & 2,400 \\
\hline $\mathrm{BF} 2 \mathrm{C} 1$ & 440 & 46 & 59 & 18 & 70 & 14 & 25 & 31 & 3,400 \\
\hline BF $2 \mathrm{C} 2$ & 240 & 29 & 42 & 10 & 70 & 9 & 16 & 13 & 1,300 \\
\hline $\mathrm{BF} 2 \mathrm{C} 3$ & 240 & 26 & 39 & 11 & 52 & 10 & 16 & 15 & 2,500 \\
\hline BF 2D2 & 680 & 54 & 63 & 16 & 40 & 13 & 31 & 33 & 1,600 \\
\hline BF 2D3 & 450 & 49 & 38 & 14 & 48 & 9 & 27 & 20 & 2,100 \\
\hline BF 2E & 760 & 52 & 65 & 12 & 23 & 12 & 29 & 37 & 490 \\
\hline $\mathrm{BF} 2 \mathrm{~N} 1$ & 760 & 54 & 53 & 11 & 21 & 12 & 30 & 37 & 400 \\
\hline $\mathrm{BF} 2 \mathrm{~N} 2^{1}$ & 670 & 43 & 34 & 8 & 12 & 8 & 23 & 22 & 350 \\
\hline $\mathrm{BF} 2 \mathrm{~N} 2^{1}$ & 680 & 44 & 37 & 8 & 15 & 8 & 27 & 24 & 370 \\
\hline $\mathrm{BF} 3 \mathrm{~A} 1$ & 230 & 27 & 31 & 6 & 45 & 8 & 16 & 11 & 1,700 \\
\hline $\mathrm{BF} 3 \mathrm{~A} 2$ & 500 & 43 & 82 & 27 & 37 & 20 & 24 & 41 & 3,600 \\
\hline BF 3B1 & 250 & 27 & 41 & 10 & 63 & 10 & 16 & 15 & 1,800 \\
\hline $\mathrm{BF}^{3} \mathrm{B2}^{2}$ & 320 & 34 & 47 & 15 & 76 & 14 & 20 & 25 & 3,900 \\
\hline $\mathrm{BF} 3 \mathrm{~B}^{2}$ & 340 & 37 & 53 & 14 & 81 & 14 & 23 & 28 & 4,000 \\
\hline BF $4 A 1$ & 490 & 42 & 56 & 16 & 65 & 11 & 24 & 24 & 3,000 \\
\hline BF 4 A 3 & 690 & 44 & 71 & 18 & 44 & 16 & 24 & 39 & 2,200 \\
\hline $\mathrm{BF} 4 \mathrm{~B} 3$ & 670 & 49 & 42 & 18 & 47 & 10 & 27 & 23 & 2,200 \\
\hline BF 4B 4 & 530 & 42 & 53 & 15 & 40 & 9 & 23 & 24 & 1,900 \\
\hline BF $4 R 1^{1}$ & 300 & 32 & 56 & 15 & 68 & 14 & 18 & 23 & 4,200 \\
\hline $\mathrm{BF} 4 \mathrm{R} 1^{1}$ & 340 & 32 & 69 & 14 & 76 & 15 & 20 & 24 & 4,200 \\
\hline BF 4R2 & 420 & 36 & 49 & 13 & 55 & 12 & 20 & 24 & 2,900 \\
\hline BF $5 A 1$ & 640 & 42 & 58 & 17 & 44 & 15 & 23 & 32 & 2,000 \\
\hline BF $5 B 1$ & 500 & 36 & 52 & 18 & 53 & 13 & 20 & 26 & 2,900 \\
\hline BF5B2 & 440 & 37 & 68 & 14 & 56 & 15 & 21 & 31 & 2,800 \\
\hline BF $5 B 3$ & 400 & 34 & 40 & 13 & 65 & 12 & 20 & 18 & 2,700 \\
\hline BF5B 4 & 1,000 & 57 & 82 & 17 & 39 & 15 & 32 & 42 & 1,300 \\
\hline $\mathrm{BF} 5 \mathrm{C} 1$ & 600 & 39 & 67 & 16 & 41 & 14 & 22 & 35 & 1,800 \\
\hline $\mathrm{BF} 5 \mathrm{C} 2$ & 490 & 32 & 49 & 16 & 59 & 13 & 18 & 23 & 2,000 \\
\hline $\mathrm{BF} 5 \mathrm{C} 3$ & 560 & 37 & 59 & 15 & 60 & 13 & 21 & 27 & 3.500 \\
\hline CRIA1 & 940 & 50 & 45 & 10 & 21 & 12 & 29 & 27 & 1,000 \\
\hline$C R 1 A 2^{2}$ & 850 & 49 & 56 & 13 & 32 & 14 & 28 & 31 & 1.600 \\
\hline CR1A2' & 890 & 46 & 55 & 13 & 32 & 13 & 29 & 32 & 1,500 \\
\hline CR1A 3 & 710 & 60 & 100 & 16 & 39 & 20 & 34 & 59 & 1,200 \\
\hline CR1A4 & 780 & 51 & 58 & 13 & 41 & 13 & 29 & 28 & 2,200 \\
\hline CR2B1 & 1,000 & 54 & 32 & 11 & 21 & 10 & 31 & 23 & 930 \\
\hline CR2B2 & 1,000 & 48 & 37 & 11 & 21 & 10 & 28 & 22 & 990 \\
\hline
\end{tabular}

${ }^{1}$ Duplicate pair. 
Table 22.- Total digestion analysis of sediment samples collected during 1985 and 1986 from the flood plains of Whitewood Creek and the Belle Fourche and Cheyenne Rivers--Continued

\begin{tabular}{|c|c|c|c|c|c|c|c|c|c|}
\hline $\begin{array}{c}\text { Sample } \\
\text { identifier }\end{array}$ & $\begin{array}{l}\text { Neodymium } \\
(\mu \mathrm{g} / \mathrm{g})\end{array}$ & $\begin{array}{l}\text { Nickel } \\
(\mu \mathrm{g} / \mathrm{g})\end{array}$ & $\begin{array}{l}\text { Lead } \\
(\mu \mathrm{g} / \mathrm{g})\end{array}$ & $\begin{array}{l}\text { Scandlium } \\
(\mu \mathrm{g} / \mathrm{g})\end{array}$ & $\begin{array}{l}\text { Strontium } \\
(\mu g / g)\end{array}$ & $\begin{array}{c}\text { Thorium } \\
(\mu \mathrm{g} / \mathrm{g})\end{array}$ & $\begin{array}{l}\text { Vanadlum } \\
(\mu g / g)\end{array}$ & $\begin{array}{l}\text { Yttrlum } \\
(\mu g / g)\end{array}$ & $\begin{array}{r}21 \text { nc } \\
(\mu g / g)\end{array}$ \\
\hline WW1A1 & 25 & 40 & 31 & 8 & 120 & 11 & 14 & 13 & 130 \\
\hline WW1A2 & 27 & 12 & 19 & 4 & 42 & 9 & 45 & 4 & 62 \\
\hline WW1A3 & 17 & 10 & 17 & 7 & 43 & 7 & 60 & 5 & 67 \\
\hline WW1B1 & 17 & 14 & 36 & 4 & 35 & 9 & 46 & 3 & 80 \\
\hline WW1B2 & 22 & 30 & 18 & 6 & 79 & 10 & 61 & 6 & 92 \\
\hline WW1B2 & 19 & 29 & 21 & 6 & 79 & 14 & 63 & 6 & 96 \\
\hline WWIC1' & 14 & 7 & 19 & 4 & 49 & 9 & 55 & 2 & 70 \\
\hline WWIC1' & 15 & 8 & 15 & 4 & 51 & 11 & 55 & 5 & 60 \\
\hline WW1C2 & 14 & 8 & 13 & 5 & 46 & 12 & 49 & 6 & 60 \\
\hline WW2A1 & 18 & 28 & 18 & 5 & 93 & 7 & 57 & 10 & 83 \\
\hline WW2A2 & 19 & 25 & 19 & 6 & 71 & 7 & 50 & 7 & 80 \\
\hline WW2A3 & 16 & 15 & 16 & 5 & 62 & 8 & 49 & 7 & 97 \\
\hline WW2A 4 & 17 & 15 & 26 & 7 & 59 & 8 & 54 & 6 & 100 \\
\hline WW2B1 & 13 & 12 & 18 & 5 & 67 & 10 & 51 & 5 & 71 \\
\hline WW2B2 & 21 & 15 & 18 & 4 & 96 & 10 & 52 & 7 & 81 \\
\hline WW2B3 & 16 & 25 & 14 & 7 & 59 & 13 & 78 & 4 & 100 \\
\hline WW2B 4 & 26 & 78 & 12 & 6 & 160 & 10 & 80 & 13 & 110 \\
\hline$B F|A|$ & 25 & 34 & 17 & 7 & 93 & 8 & 70 & 17 & 82 \\
\hline BF1A2 & 17 & 23 & 16 & 4 & 80 & 7 & 48 & 11 & 66 \\
\hline $\mathrm{BF} 1 \mathrm{~A} 3$ & 19 & 18 & 13 & 5 & 74 & 10 & 45 & 7 & 80 \\
\hline BF1B2 & 23 & 35 & 30 & 8 & 110 & 9 & 81 & 13 & 110 \\
\hline BE 1B31 & 11 & 11 & 10 & 4 & 60 & 6 & 38 & 4 & 54 \\
\hline $\mathrm{BF} \backslash \mathrm{C} 1^{\prime}$ & 13 & 18 & 13 & 6 & 54 & 8 & 60 & 8 & 87 \\
\hline $\mathrm{BF} 1 \mathrm{C} 1^{1}$ & 15 & 18 & 15 & 6 & 56 & 6 & 62 & 8 & 90 \\
\hline BF1C2 & 25 & 31 & 49 & 9 & 120 & 11 & 92 & 13 & 130 \\
\hline $\mathrm{BF} 2 \mathrm{~A} 1$ & 15 & 12 & 13 & 6 & 64 & 7 & 59 & 6 & 62 \\
\hline$B F 2 A 2$ & 20 & 22 & 18 & 8 & 120 & 10 & 80 & 9 & 140 \\
\hline $\mathrm{BF} 2 \mathrm{B1}$ & 17 & 10 & 12 & 5 & 63 & 7 & 49 & 5 & 61 \\
\hline BF 2B2 & 18 & 24 & 15 & 6 & 110 & 9 & 63 & 10 & 97 \\
\hline $\mathrm{BF} 2 \mathrm{C} 1$ & 23 & 31 & 22 & 8 & 90 & 9 & 85 & 13 & 100 \\
\hline $\mathrm{BF} 2 \mathrm{C} 2$ & 14 & 13 & 14 & 5 & 61 & 7 & 48 & 4 & 60 \\
\hline $\mathrm{BF} 2 \mathrm{C} 3$ & 13 & 18 & 11 & 5 & 57 & 5 & 47 & 5 & 68 \\
\hline BF 2D2 & 27 & 66 & 18 & 8 & 110 & 9 & 98 & 22 & 97 \\
\hline $\mathrm{BF} 2 \mathrm{D} 3$ & 23 & 25 & 19 & 5 & 95 & 9 & 56 & 12 & 78 \\
\hline BF 2E & 26 & 31 & 19 & 9 & 140 & 9 & 100 & 19 & 83 \\
\hline $\mathrm{BF} 2 \mathrm{~N} 1$ & 27 & 30 & 17 & 8 & 150 & 9 & 97 & 18 & 76 \\
\hline $\mathrm{BF} 2 \mathrm{~N} 2^{\prime}$ & 22 & 17 & 13 & 5 & 130 & 7 & 52 & 14 & 39 \\
\hline $\mathrm{BF} 2 \mathrm{~N} 2^{1}$ & 24 & 19 & 13 & 6 & 140 & 7 & 58 & 15 & 43 \\
\hline$B E 3 A 1$ & 13 & 7 & 16 & 5 & 120 & 8 & 47 & 7 & 49 \\
\hline$B F 3 A 2$ & 22 & 66 & 19 & 13 & 110 & 12 & 170 & 14 & 130 \\
\hline BF 3B1 & 14 & 14 & 13 & 6 & 61 & 8 & 59 & 7 & 70 \\
\hline $\mathrm{BF} 3 \mathrm{~B} 2^{1}$ & 17 & 26 & 10 & 7 & 87 & 9 & 74 & 9 & 93 \\
\hline $\mathrm{BF} 3 \mathrm{~B} 2^{1}$ & 17 & 27 & 14 & 7 & 87 & 7 & 75 & 9 & 100 \\
\hline BF4AI & 21 & 28 & 20 & 7 & 94 & 9 & 76 & 12 & 90 \\
\hline$B F 4 A 3$ & 21 & 45 & 20 & 10 & 120 & 8 & 120 & 15 & 110 \\
\hline$B F 4 B 3$ & 23 & 36 & 15 & 6 & 96 & 9 & 69 & 13 & 84 \\
\hline BF 4B 4 & 20 & 39 & 16 & 6 & 93 & 7 & 66 & 10 & 79 \\
\hline$B F^{4} 1^{\prime}$ & 15 & 38 & 13 & 7 & 73 & 8 & 70 & 8 & 96 \\
\hline$B F 4 R 1^{\prime}$ & 14 & 38 & 15 & 7 & 82 & 8 & 76 & 8 & 100 \\
\hline$B F 4 R 2$ & 17 & 23 & 14 & 7 & 96 & 7 & 69 & 8 & 87 \\
\hline BF $5 \mathrm{~A} 1$ & 23 & 38 & 16 & 9 & 120 & 11 & 110 & 15 & 110 \\
\hline BF5B1 & 19 & 35 & 16 & 8 & 94 & 9 & 90 & 12 & 110 \\
\hline BF5B2 & 19 & 28 & 19 & 9 & 87 & 8 & 97 & 11 & 100 \\
\hline BF5B3 & 18 & 22 & 14 & 6 & 99 & 8 & 65 & 9 & 91 \\
\hline BF5B 4 & 28 & 41 & 23 & 11 & 150 & 10 & 130 & 19 & 120 \\
\hline $\mathrm{BF} 5 \mathrm{C} 1$ & 20 & 39 & 20 & 10 & 140 & 10 & 120 & 16 & 100 \\
\hline BF5C2 & 17 & 24 & 17 & 8 & 110 & 8 & 89 & 11 & 96 \\
\hline BF 5 C 3 & 19 & 33 & 19 & 7 & 99 & 7 & 83 & 11 & 96 \\
\hline CRIA1 & 24 & 22 & 17 & 6 & 290 & 9 & 66 & 16 & 64 \\
\hline CRIA2' & 24 & 29 & 18 & 7 & 220 & 9 & 82 & 16 & 79 \\
\hline CR1A2' & 22 & 28 & 20 & 8 & 240 & 9 & 87 & 16 & 82 \\
\hline CR1A3 & 30 & 45 & 22 & 14 & 180 & 12 & 160 & 20 & 120 \\
\hline CRIA4 & 26 & 24 & 18 & 7 & 220 & 10 & 76 & 15 & 79 \\
\hline CR2B1 & 27 & 25 & 17 & 5 & 200 & 8 & 62 & 16 & 66 \\
\hline CR2B2 & 24 & 23 & 17 & 5 & 200 & 7 & 60 & 15 & 66 \\
\hline
\end{tabular}

'Duplicate pair. 


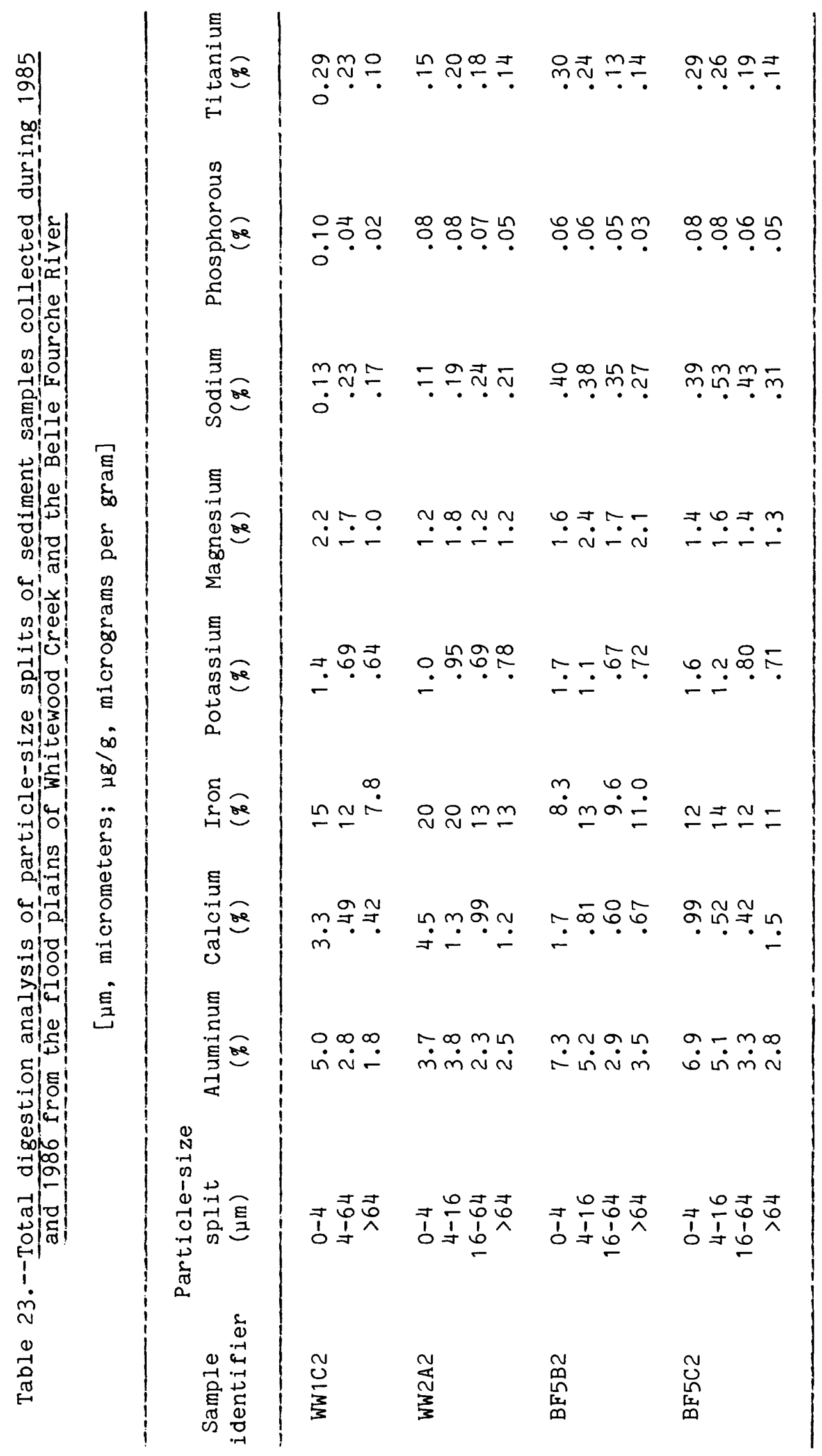




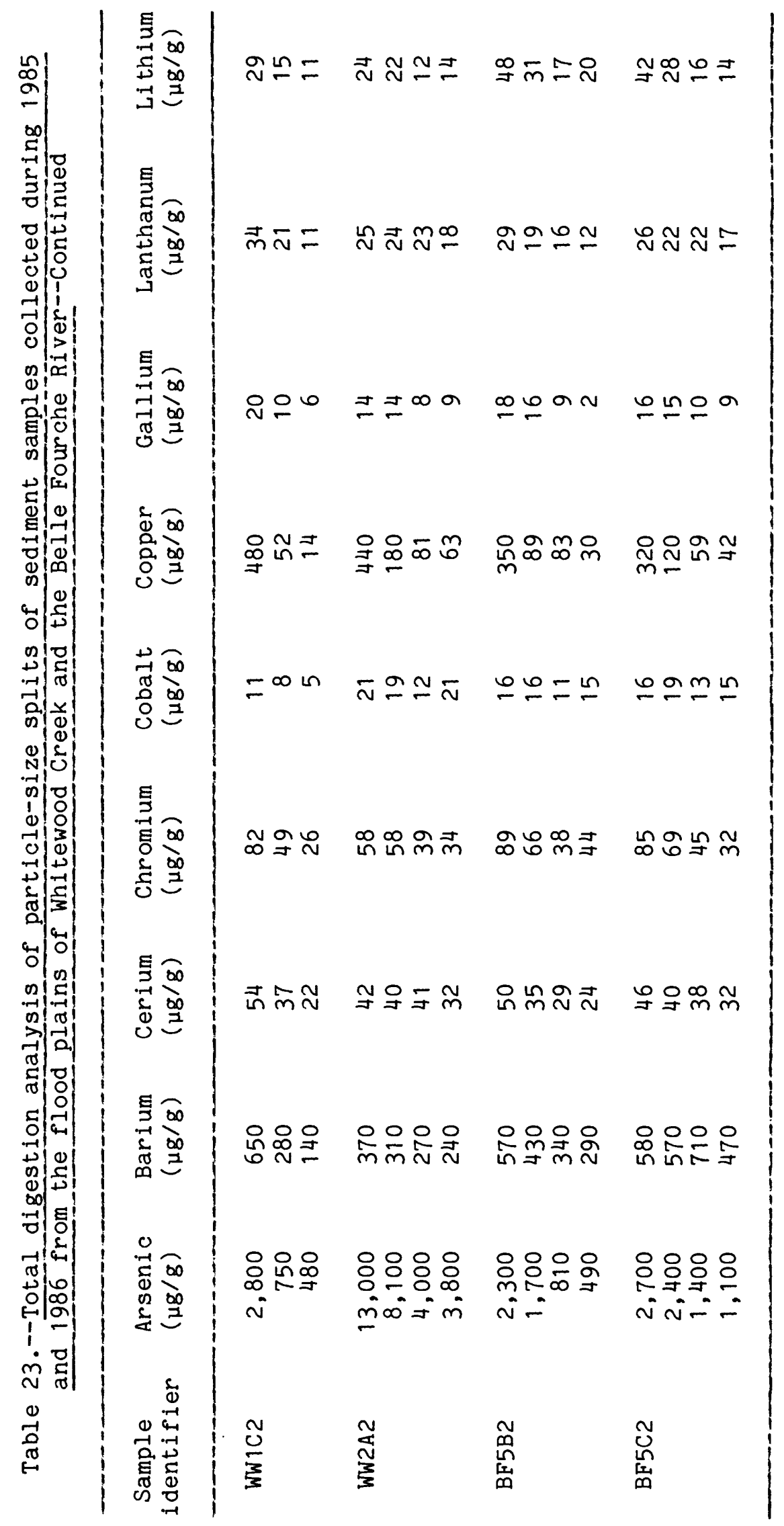




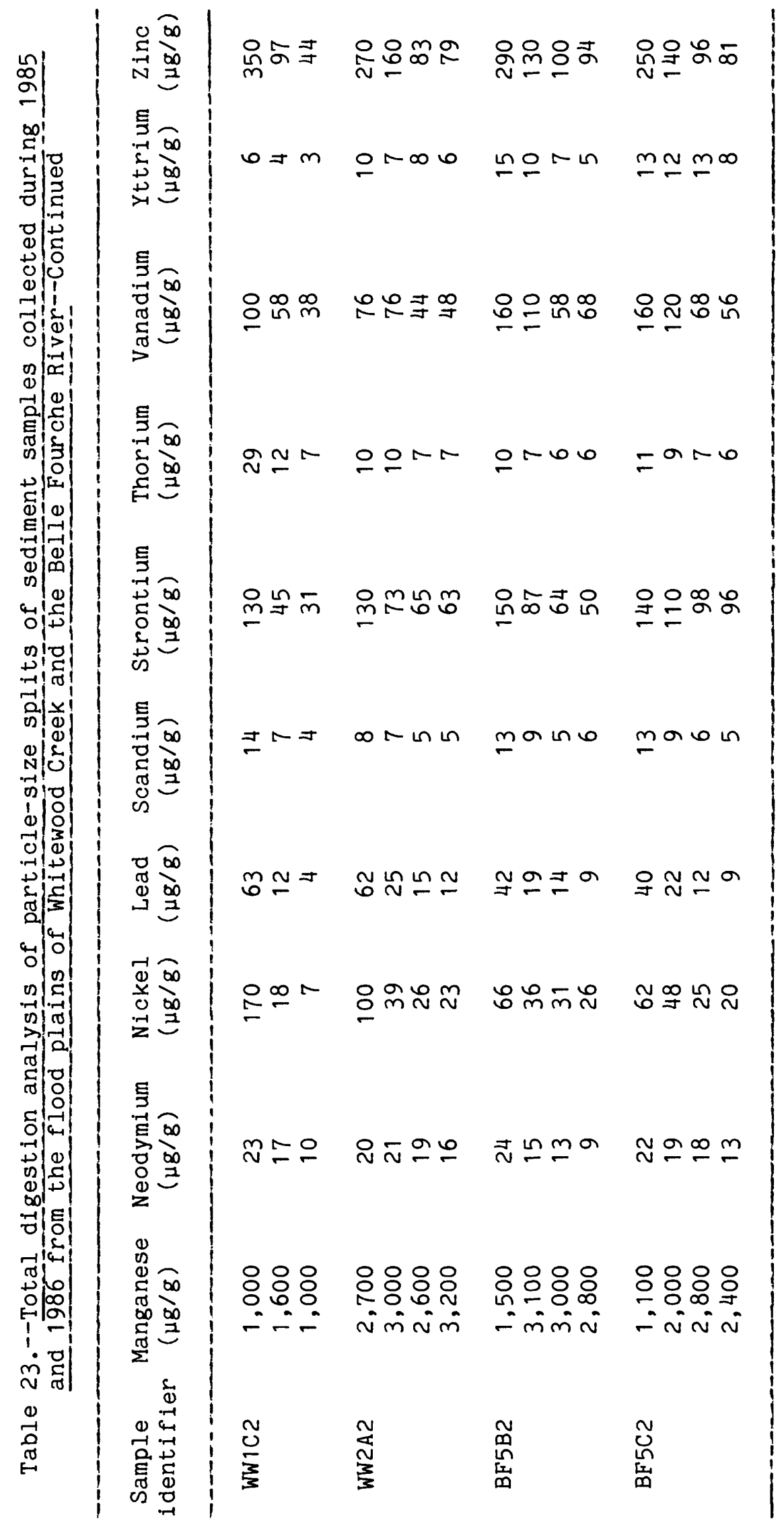




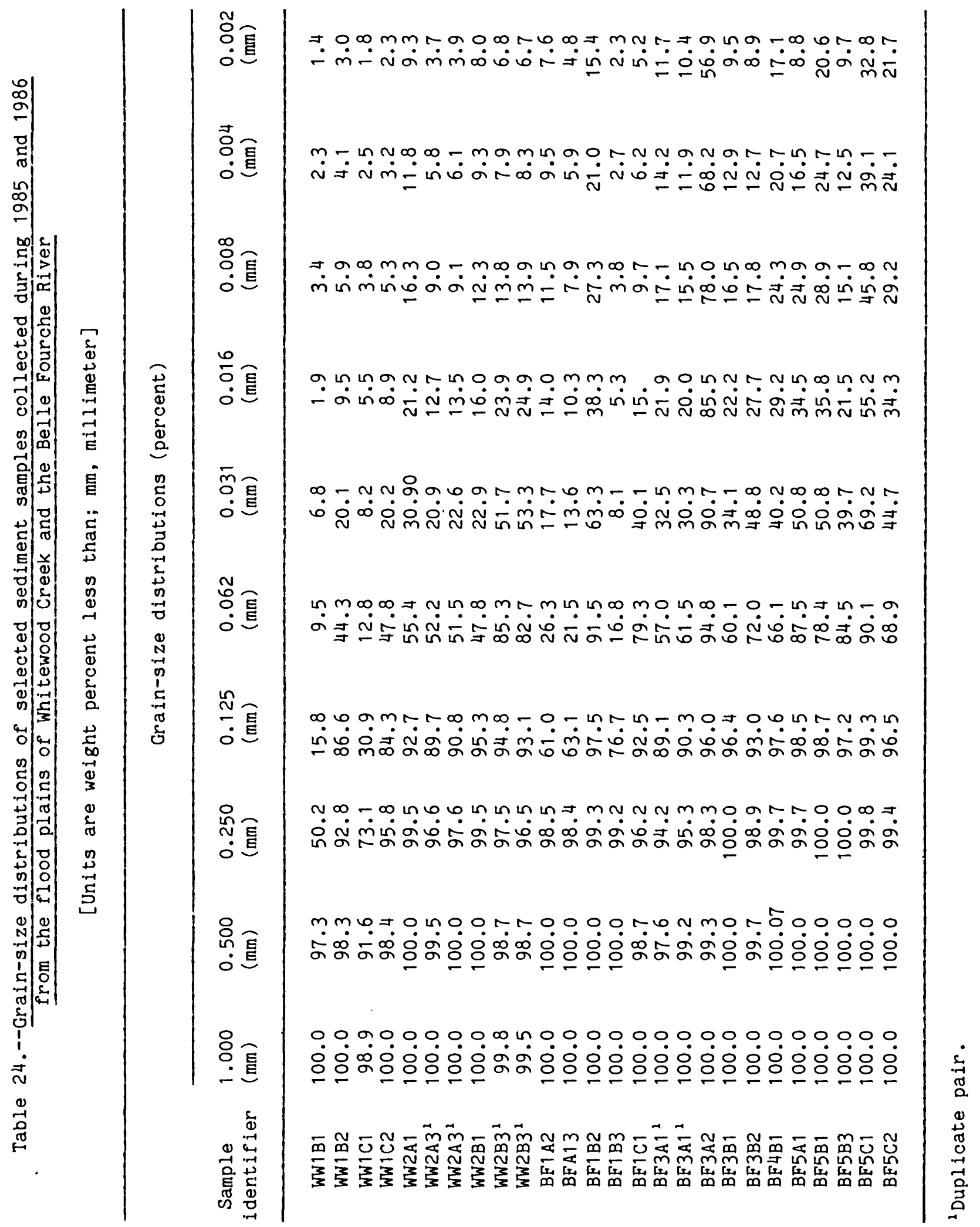




\section{Chapter C. Trace-Element Geochemistry of Bottom Sediment Samples Collected from Whitewood Creek and the Belle Fourche and Cheyenne Rivers}

By Kimball E. Goddard, Arthur J. Horowitz, and Kent A. Elrick

\section{SAMPLE COLLECTION AND HANDLING PROCEDURES}

Bottom sediment materials were collected from 15 sites along Whitewood Creek and the Belle Fourche and Cheyenne Rivers between August 6 and 13, 1985. The site locations are shown in figures 1 and 2 and listed in table 1. Additional site information is provided in table 25.

Samples were collected by scooping bottom sediments into a plastic 64-um sieve-bucket using a small plastic shovel. Individual scoops were collected randomly from 5 to 20 locations within the wetted perimeter of the active stream channel at each location and composited in the sieve. The materials were washed with native stream water and the material passing the sieve was retained. All materials not passing the sieve were discarded. Field sieving was necessary to remove coarse gravel and cobbles, living and dead plant materials, and to insure a sufficient amount of less than $64 \mu \mathrm{m}$ material to allow analysis. Many of the Whitewood Creek sites had rock and cobble bottoms that precluded whole-volume bottom-sediment sample collection. The sedimentwater slurry retained after sieving was pored into wide-mouth jars and chilled on ice to $4{ }^{\circ} \mathrm{C}$ for transport to the sample preparation laboratory. All implements used during sample collection were washed in 3 percent nitric acid solution and rinsed in deionized and native water before sampling.

The bottom material samples were concentrated by centrifugation. A Beckman model J2-21 centrifuge with a JA-10 fixed-angle rotor was used to spin $500 \mathrm{~mL}$ subsamples at $5,000 \mathrm{rpm}$ for 5 minutes. The subsamples were subsequently composted into a single 500-mL centrifuge bottle. The freeze-dried samples, in the original centrifuge bottles, were kept at room temperature until analysis.

\section{ANALYTICAL PROCEDURES AND RESULTS}

Subsamples for total arsenic and metals analysis, total recoverable arsenic and metals analysis, and total organic carbon analysis were obtained from the whole samples following homogenization of the powdered, freeze-dried sample.

Total digestion analysis for iron, manganese, aluminum, titanium, arsenic, antimony, cadmium, chromium, cobalt, copper, lead, mercury, nickel, selenium, and zinc were conducted on subsamples by Arthur J. Horowitz and Kent Elrick, U.S. Geological Survey, Doraville, Georgia. Procedures followed were those of Horowitz and Elrick (1985) and Elrick and Horowitz (1985; 1986). For all elements except mercury, samples were digested with a combination of $\mathrm{HF} / \mathrm{HC} 10_{4} / \mathrm{HNO}_{3}$ acids at $200{ }^{\circ} \mathrm{C}$. For mercury, samples were digested with LeForte aqua regia at $100^{\circ} \mathrm{C}$. Quantitation was by atomic absorption spectrophotometry. Analytical results are listed in table 26 . 


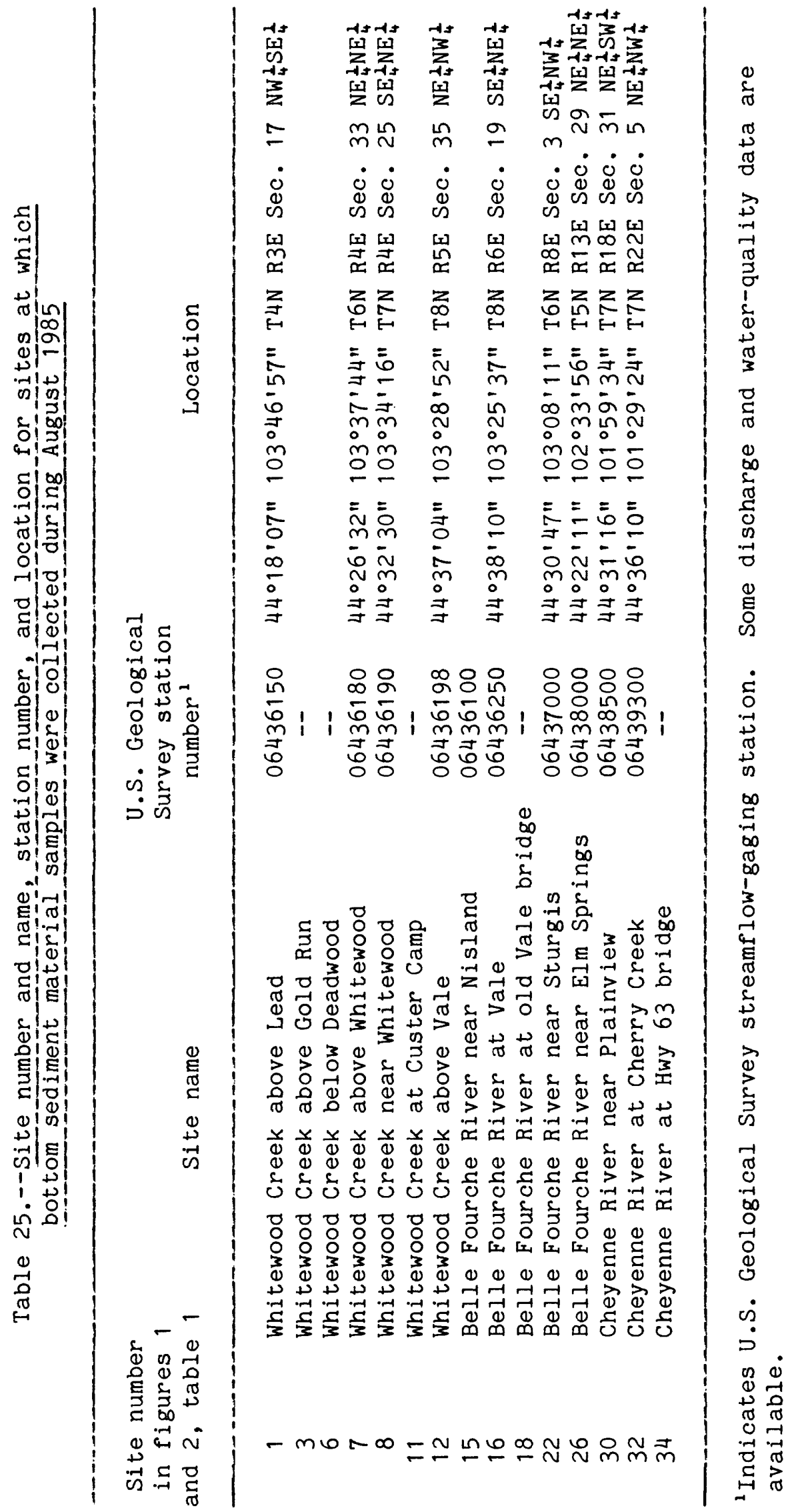




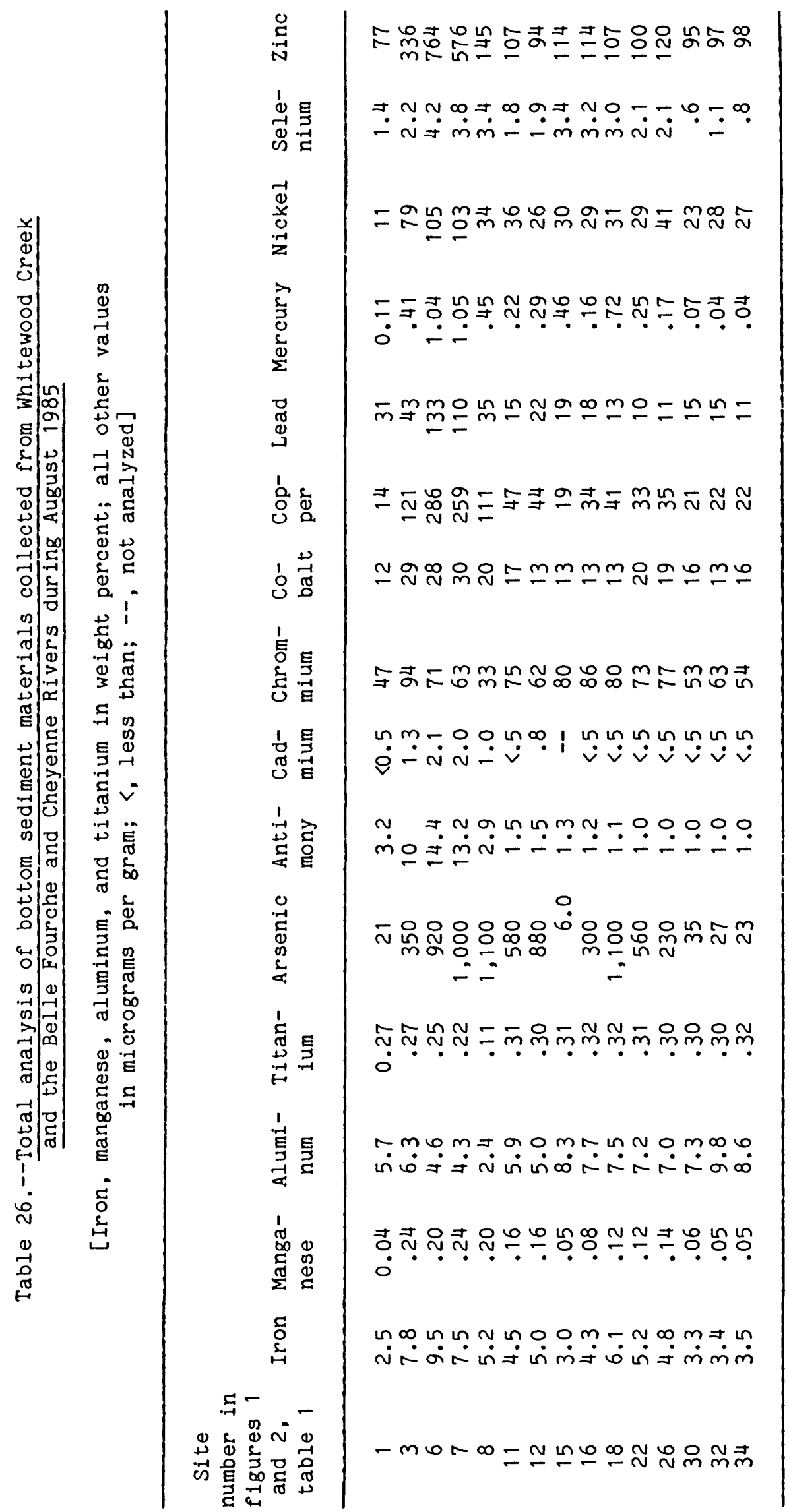


Total recoverable digestion analysis for iron, manganese, aluminum, arsenic, antimony, cadmium, chromium, cobalt, copper, lead, mercury, nickel, selenium, and zinc were conducted on subsamples by the U.S. Geological Survey laboratory at Arvada, Colorado. Procedures followed were standard U.S. Geological Survey procedures for the analysis of bottom materials as described by Skougstad and others (1970). For elements except arsenic, antimony, mercury, and selenium, samples were digested with 6 Molar HC1 at $100{ }^{\circ} \mathrm{C}$. For arsenic and antimony, samples were digested with a combination of $\mathrm{H}_{2} \mathrm{SO}_{4}$ and $\mathrm{HNO}_{3}$ at $100{ }^{\circ} \mathrm{C}$. For mercury, samples were digested with a potassium permanganate/potassium persulfate acid solution at $95^{\circ} \mathrm{C}$ followed by reduction with stannous chloride. Finally, selenium was recovered using potassium permanganate at $100{ }^{\circ} \mathrm{C}$, followed by digestion with $6 \mathrm{M} \mathrm{HC} 1$ at $100^{\circ} \mathrm{C}$. Quantitation was by atomic absorption. Analytical results are listed in table 27.

Analyses for total organic carbon were conducted on subsamples by the U.S. Geological Survey laboratory at Arvada, Colorado. Procedures followed were standard U.S. Geological Survey procedures for the analysis of bottom materials as described by Wershaw and others (1983). Organic carbon is recovered from the sample using a persulfate oxidation process. Analytical results are also listed in table 27.

Subsamples for particle-size analysis were obtained from the whole samples following homogenization of the powdered, freeze-dried sample. Sample analysis was performed by the U.S. Geological Survey sediment laboratory in Iowa City, Iowa. Procedures followed were standard U.S. Geological Survey procedures for the enumeration of fluvial sediment as described by Guy (1969). Analysis results are listed in table 28 . 


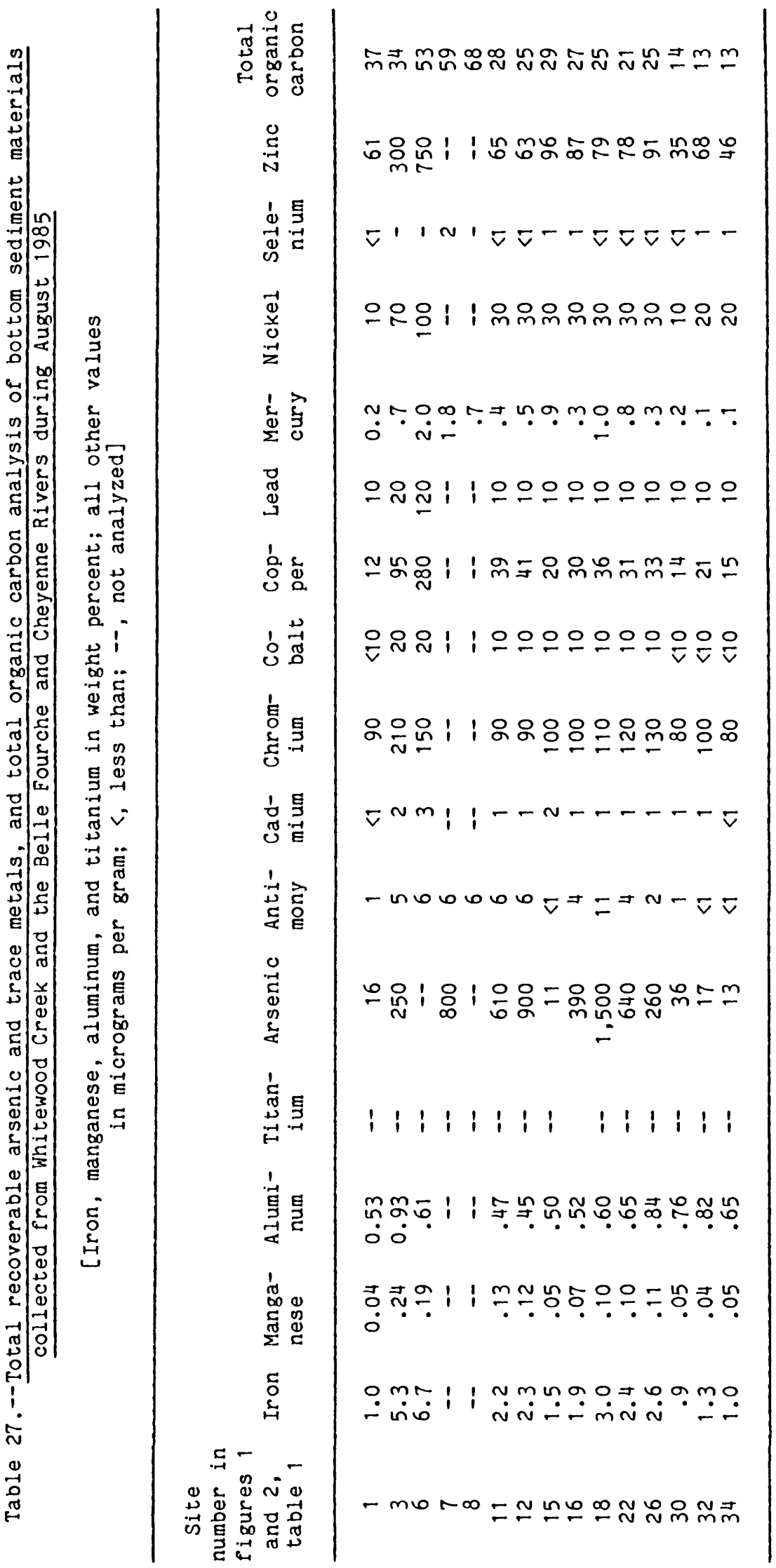


Table 28.--Particle-size analysis of bottom sediment materials collected from Whitewood Creek and the Belle Fourche and Cheyenne Rivers during August 1985

[Samples field sieved to $<0.064 \mathrm{~mm}$ (millimeter)]

\begin{tabular}{|c|c|c|c|c|c|c|}
\hline $\begin{array}{l}\text { Site } \\
\text { number in } \\
\text { figures } 1 \\
\text { and } 2, \\
\text { table } 1\end{array}$ & $\begin{array}{l}\text { Sieve } \\
\text { diameter } \\
\text { \% finer } \\
\text { than } \\
0.064 \mathrm{~mm}\end{array}$ & $\begin{array}{l}\text { Fall } \\
\text { diameter } \\
\text { \% finer } \\
\text { than } \\
0.032 \mathrm{~mm}\end{array}$ & $\begin{array}{l}\text { Fall } \\
\text { diameter } \\
\% \text { finer } \\
\text { than } \\
0.016 \mathrm{~mm}\end{array}$ & $\begin{array}{l}\text { Fall } \\
\text { diameter } \\
\text { \% finer } \\
\text { than } \\
0.008 \mathrm{~mm}\end{array}$ & $\begin{array}{c}\text { Fall } \\
\text { diameter } \\
\text { \% finer } \\
\text { than } \\
0.004 \mathrm{~mm}\end{array}$ & $\begin{array}{l}\text { Fall } \\
\text { diameter } \\
\text { \% finer } \\
\text { than } \\
0.002 \mathrm{~mm}\end{array}$ \\
\hline 1 & 100 & 74 & 48 & 36 & 28 & 21 \\
\hline 3 & 100 & 85 & 47 & 27 & 15 & 7 \\
\hline 6 & 100 & 78 & 56 & 36 & 19 & 11 \\
\hline 7 & 100 & 81 & 58 & 37 & 19 & 9 \\
\hline 8 & 100 & 87 & 62 & 37 & 15 & 1 \\
\hline 11 & 100 & 84 & 63 & 46 & 33 & 20 \\
\hline 12 & 100 & 85 & 65 & 52 & 41 & 31 \\
\hline 15 & 100 & 90 & 79 & 69 & 54 & 40 \\
\hline 16 & 100 & 89 & 78 & 67 & 52 & 38 \\
\hline 18 & 100 & 82 & 67 & 55 & 43 & 33 \\
\hline 22 & 100 & 91 & 77 & 65 & 53 & 41 \\
\hline 26 & 100 & 96 & 84 & 70 & 57 & 43 \\
\hline 30 & 100 & 96 & 89 & 76 & 66 & 56 \\
\hline 32 & 100 & 98 & 85 & 68 & 56 & 46 \\
\hline 34 & 100 & 92 & 84 & 72 & 62 & 53 \\
\hline
\end{tabular}




\section{REFERENCES}

Elrick, K.A., and Horowitz, A.J., 1985, Analysis of rocks and sediments for arsenic, antimony, and selenium by wet digestion and hydride generation atomic absorption: U.S. Geological Survey Open-File Report 85-447, 14 p.

1986, Analysis of rocks and sediments for mercury by wet digestion and flameless cold vapor atomic absorption, in Varian Instruments at Work (in press).

Guy, H.P., 1969, Laboratory theory and methods for sediment analysis: U.S. Geological Survey Techniques of Water-Resources Investigations, Book 5 , Chap. C1, $58 \mathrm{p}$.

Horowitz, A.J., and Elrick, K.A., 1985, Multielement analysis of rocks and sediments by wet digestion and atomic absorption spectroscopy (unpublished draft).

Skougstad, M.W., Dishman, M.J., Friedman, L.C., Erdmann, D.E., and Duncan, S.S., 1979, Methods for determination of inorganic substances in water and fluvial sediments, U.S. Geological Survey Techniques of waterResources Investigations, Book 5, Chap. A1, $626 \mathrm{p}$.

Wershaw, R.L., Fishman, M.J., Grabbe, R.R., and Lowe, L.E., 1983, Methods for the determination of organic substances in water and fluvial sediments: U.S. Geological Survey Techniques of Water-Resources Investigations, Book 5, Chap. A3. 


\section{Chapter D. Trace-Element Geochemistry of Sediment Cores Collected from the Cheyenne River Arm of Lake Oahe}

By Arthur J. Horowitz, Kent A. Elrick, and Edward Callender

\section{SAMPLE COLLECTION AND HANDLING PROCEDURES}

During August 1985, a series of six cores were obtained along the line of the Old Cheyenne River channel between the present mouth of the Cheyenne River, through the Cheyenne River arm of Lake Oahe, to the lake proper (fig. 3). The line of the old river channel was located by fathometer and was selected for two reasons: (1) To obtain, if possible, samples from the channel deposited prior to the closing of the Missouri River by the Oahe Dam in 1958; and (2) to avoid contact with the numerous tree trunks which still extend above the sediment from the former river banks, which were submerged after 1958. Cores were obtained using a Benthos gravity corer with either a 4- or 8-ft (approximately 122 or $244 \mathrm{~cm}$ ) plastic-lined barrel. Several cores were obtained at each site for different purposes. Those destined for tracemetal analysis were extruded on site using a series of rubber stoppers inserted into the plastic core liner. Specific sections were excised from each core. Usually the entire core was sampled; care was taken to limit samples to single bands in the cores. Once a section was obtained, it was placed immediately in a large-mouth plastic bottle, chiiled on ice to $4{ }^{\circ} \mathrm{C}$ in a cooler, and shipped to Atlanta, Georgia, for subsequent processing and chemical analysis. Individual sample bottles were kept refrigerated until processing could begin.

\section{ANALYTICAL PROCEDURES AND RESULTS}

Individual sample bottles were removed from the refrigerator and thoroughly homogenized using acid-rinsed glass rods. Subsamples were then removed, placed in porcelain evaporating dishes, and freeze dried. The remaining sample was returned to the refrigerator for storage. Chemical analyses for iron, manganese, aluminum, titanium, copper, zinc, cadmium, lead, nickel, cobalt, chromium, arsenic, antimony, selenium, and mercury were carried out on freeze-dried samples following the procedures of Horowitz and Elrick (1985) and Elrick and Horowitz (1985, 1987). For all elements other than arsenic, antimony, selenium, and mercury, 500-mg samples were digested with a combination of $\mathrm{HF}-\mathrm{HC}^{\circ} \mathrm{O}_{4}-\mathrm{HNO}_{3}$ acids in teflon beakers at $200{ }^{\circ} \mathrm{C}$; the resulting salts were brought into solution with $50 \mathrm{~mL}$ of 2 percent $\mathrm{HCL}$. Quantification was by flame atomic absorption spectrophotometry using a Varian model AA-975 and mixed-salt standards. The determination of arsenic, antimony, and selenium used the same digestion, but final solutions were made up in 50 percent HCL. Quantification was by hydride generation and atomic absorption spectrophotometry using a Varian VGA-76 hydride generator and the AA-975. Mercury was determined using a 500-mg sample digested with LeFort aqua regia at $100^{\circ} \mathrm{C}$ with quantification by a cold-vapor technique using the VGA-76 system in conjunction with the AA-975. Precision and bias (generally better than $\pm 10 \%$ ) were monitored by replicate analyses of selected samples and by the concomitant digestion and analysis of National Bureau of Standards sediment and U.S. Geological Survey rock standards. Total volatile solids (loss on ignition) was used as a measure of the organic carbon, sulfur and 


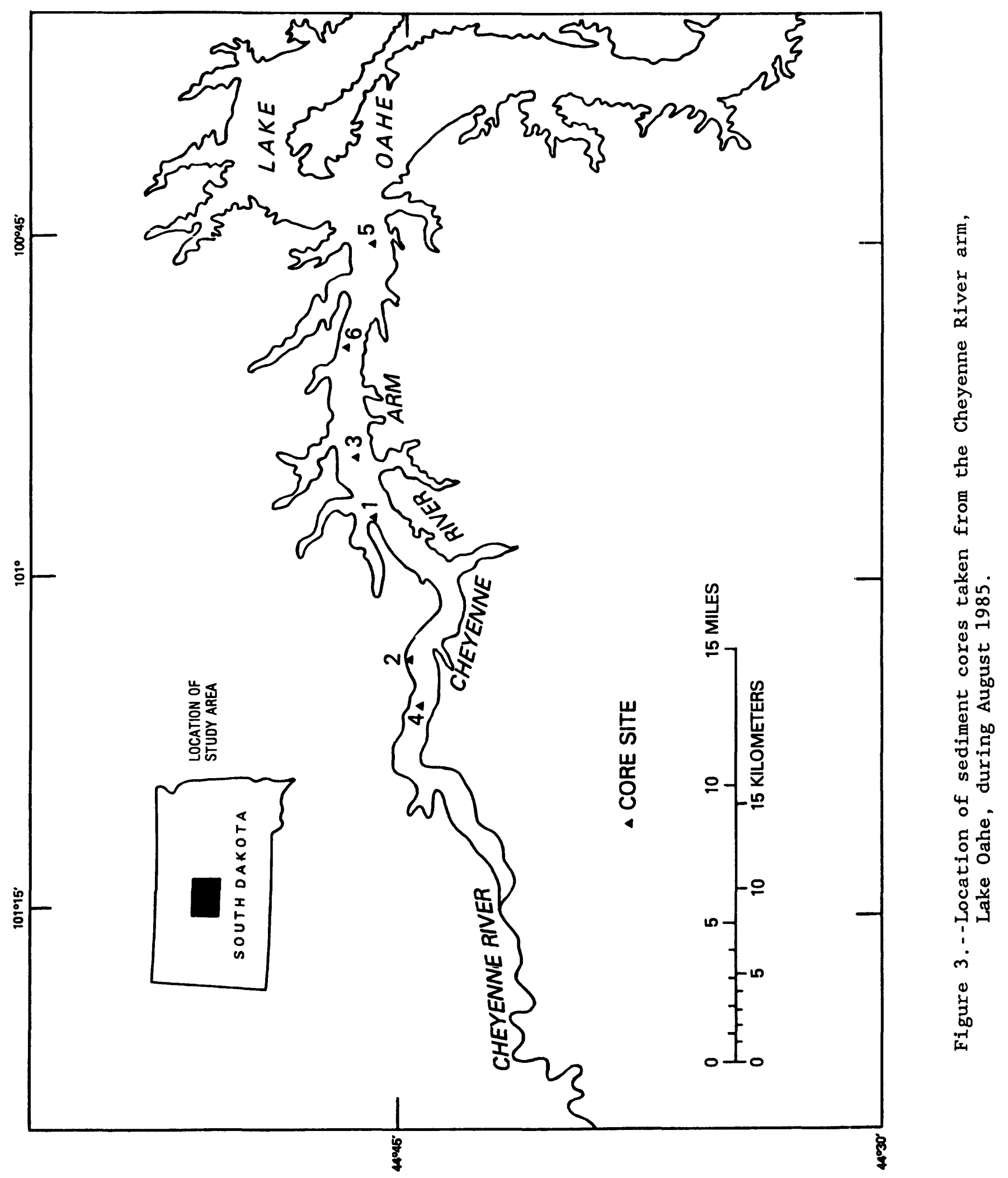


water of hydration in each sample and was determined following the procedures outlined in Skougstad and others (1979). This entailed the ignition of preweighed samples and their subsequent re-weighing after 1 hour at $550{ }^{\circ} \mathrm{C}$ in a muffle furnace. Analytical results for all the samples are given in table 29; cadmium has been omitted because almost all the samples had concentrations below the detection limit of $0.5 \mu \mathrm{g} / \mathrm{g}$.

\section{REFERENCES}

Elrick, K.A., and Horowitz, A.J., 1985, Analysis of rocks and sediments for arsenic, antimony, and selenium by wet digestion, atomic absorption spectroscopy, and hydride generation, in Varian Instruments at Work, $\mathrm{AA}-56,5 \mathrm{p}$.

--.- 1987, Analysis of rocks and sediments for mercury by wet digestion and flameless cold vapor atomic absorption, in Varian Instruments at Work, $\mathrm{AA}-72,5 \mathrm{p}$.

Horowitz, A.J., and Elrick, K.A., 1985, Multielement analysis of rocks and sediments by wet digestion and atomic absorption spectroscopy, in Varian Instruments at Work, AA-47, $7 \mathrm{p}$.

Skougstad, M.W., Fishman, M.J., Friedman, L.C., Erdmann, D.E., and Duncan, S.S., 1979, Methods for determination of inorganic substances in water and fluvial sediments, U.S. Geological Survey Techniques of WaterResources Investigation, Book 5, Chap. A1, 626 p. 
Table 29.--Chemical composition or sediment core samples taken from the Cheyenne River arm of Lake Oahe during August 1985

[Alumlnum, Iron, manganese, tItanlum, and LOI In welght percent; all others values in micrograms per gram; cm, centlmetera]

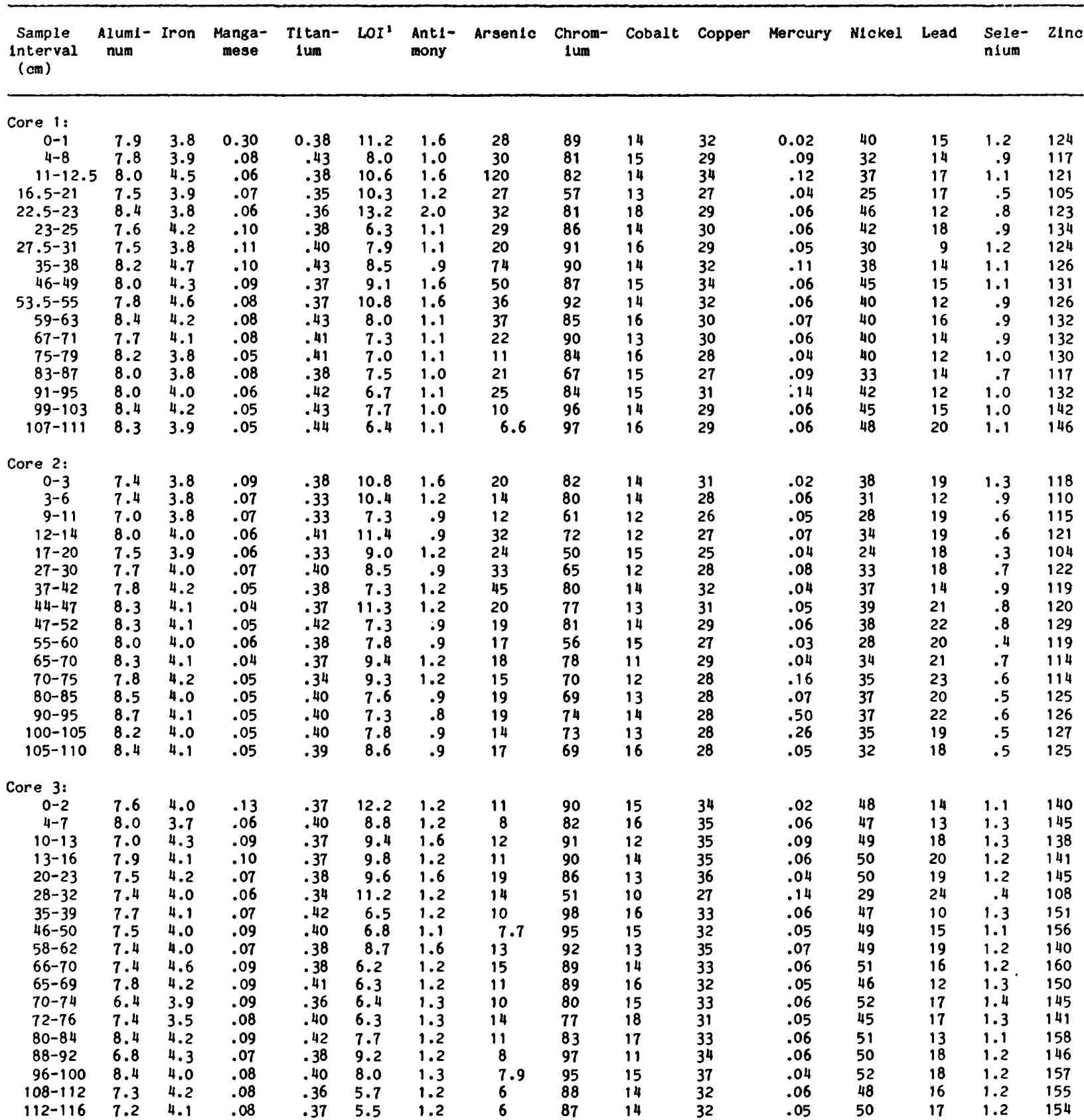


Table 29.--Chemlcal composition of sediment core samples taken from the

Cheyenne River arm of Lake Oahe during August 1985--Continued

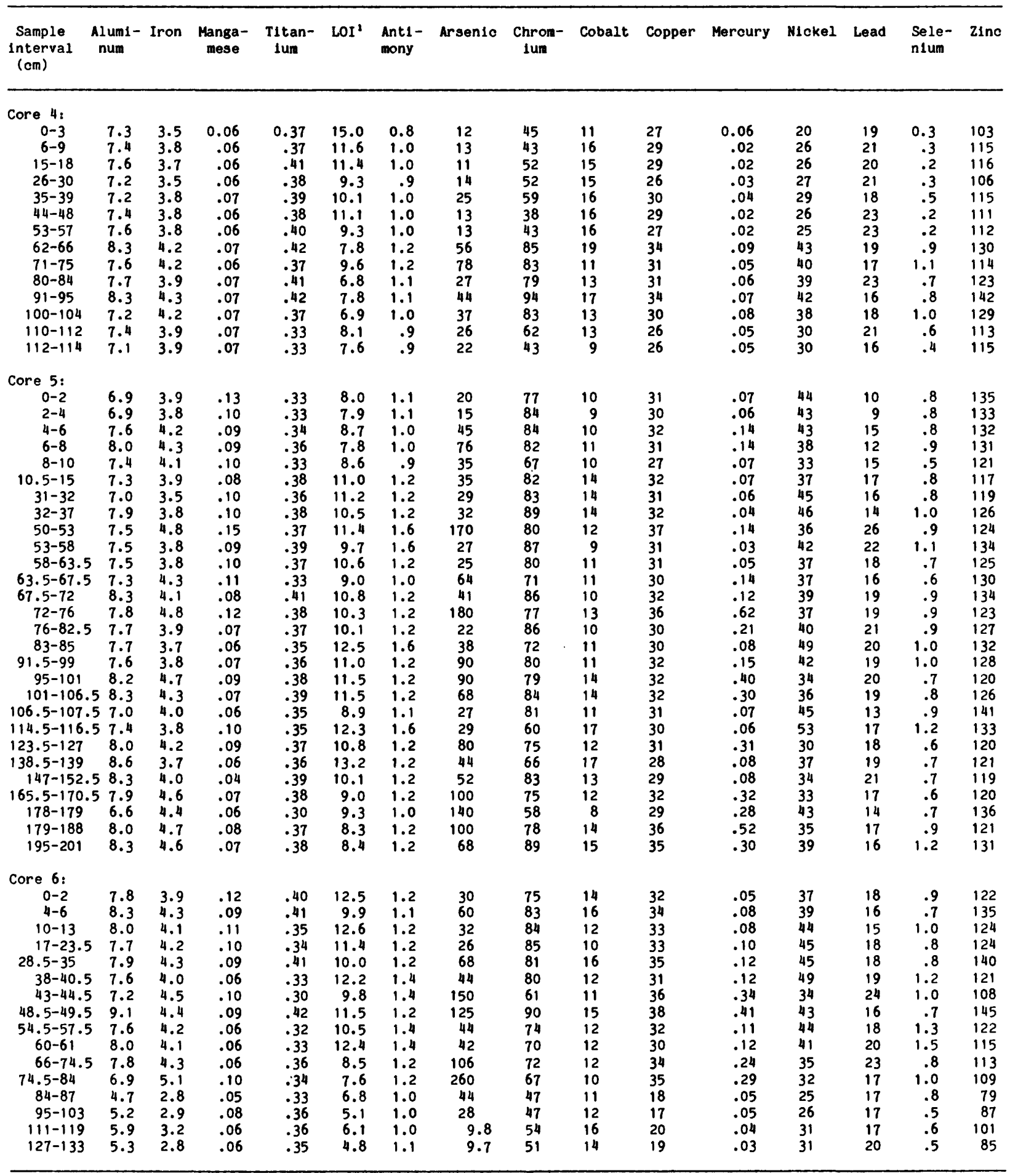

'Loss on ignition, total volatile solids. 
Chapter E. Discharge and Surface-Water Quality at Selected Sites on Whitewood Creek and the Belle Fourche and Cheyenne Rivers

By William R. Roddy and Thomas E. McKallip

METHODS

Discharge Data

Continuous discharge data were obtained at 4 streamflow-gaging stations, and instantaneous discharge data were obtained at 16 miscellaneous waterquality sites (figs. 1 and 2, table 1). Discharge data were obtained following procedures by Buchanan and Somers (1969, 1982), Craig (1983), and Kennedy (1983, 1984).

Discharge measurements were calculated by summing the products of width, depth, and velocity at 20 to 40 sub-sections along a cross section of the stream. Depths were measured with a graduated wading rod or with sounding weights suspended by a metal cable. Water velocities were determined using either a Price "AA" or a pygmy meter. Widths were measured based on the midpoints between sub-sections. In a few instances, because of ice effects or fast-rising stages, measurements were made with fewer sub-sections or with less time for velocity measurements than published guidelines. In every case, these measurements were checked against the water stage-discharge rating and were found to be acceptably accurate.

Calculation of daily mean discharges (tables 30 through 33 ) at continuous-record gages was based on water stages recorded every 15 minutes and on the water stage-discharge ratings. Water stages were sensed by a bubble-gage manometer and punched on paper tape by a digital recorder. Water stage-discharge ratings were derived from the relation between monthly and high-flow discharge measurements and corresponding water stages. Records are accurate except for periods in winter when ice covered the streams. Records during periods of ice cover have been adjusted and are considered poor. 
Table 30.--Daily mean discharge, in cubic feet per second, for Whitewood Creek above Whitewood, S. Dak.

DISCHARGE, CUBIC FEET PER SECOND, WATER YEAR OCTOBER 1984 TO SEPTEMBER 1985 MEAN VALUES

\begin{tabular}{|c|c|c|c|c|c|c|c|c|c|c|c|c|}
\hline DAY & OCT & NOV & DEC & JAN & FEB & MAR & APR & MAY & JUN & JUL & AUG & SEP \\
\hline $\begin{array}{l}1 \\
2 \\
3 \\
4 \\
5\end{array}$ & $\begin{array}{l}14 \\
15 \\
15 \\
18 \\
19\end{array}$ & $\begin{array}{l}14 \\
14 \\
14 \\
14 \\
14\end{array}$ & $\begin{array}{l}12 \\
11 \\
10 \\
10 \\
11\end{array}$ & $\begin{array}{l}10 \\
11 \\
15 \\
18 \\
20\end{array}$ & $\begin{array}{r}8.0 \\
8.5 \\
9.0 \\
9.5 \\
10\end{array}$ & $\begin{array}{l}13 \\
12 \\
12 \\
12 \\
12\end{array}$ & $\begin{array}{l}19 \\
21 \\
26 \\
28 \\
22\end{array}$ & $\begin{array}{l}17 \\
16 \\
16 \\
16 \\
16\end{array}$ & $\begin{array}{l}13 \\
13 \\
15 \\
13 \\
14\end{array}$ & $\begin{array}{l}12 \\
11 \\
11 \\
11 \\
10\end{array}$ & $\begin{array}{l}9.6 \\
9.4 \\
9.3 \\
9.6 \\
9.0\end{array}$ & $\begin{array}{c}10 \\
11 \\
12 \\
10 \\
9.7\end{array}$ \\
\hline $\begin{array}{r}6 \\
7 \\
8 \\
9 \\
10\end{array}$ & $\begin{array}{l}22 \\
18 \\
19 \\
17 \\
17\end{array}$ & $\begin{array}{l}13 \\
15 \\
14 \\
14 \\
14\end{array}$ & $\begin{array}{l}13 \\
16 \\
17 \\
18 \\
18\end{array}$ & $\begin{array}{l}20 \\
18 \\
17 \\
16 \\
15\end{array}$ & $\begin{array}{l}10 \\
11 \\
11 \\
12 \\
12\end{array}$ & $\begin{array}{l}13 \\
14 \\
18 \\
16 \\
15\end{array}$ & $\begin{array}{l}20 \\
19 \\
19 \\
20 \\
21\end{array}$ & $\begin{array}{l}15 \\
15 \\
16 \\
15 \\
15\end{array}$ & $\begin{array}{l}14 \\
13 \\
13 \\
12 \\
17\end{array}$ & $\begin{array}{l}10 \\
10 \\
9.1 \\
9.2 \\
10\end{array}$ & $\begin{array}{l}9.5 \\
9.8 \\
9.2 \\
9.3 \\
9.1\end{array}$ & $\begin{array}{l}9.6 \\
9.2 \\
10 \\
11 \\
11\end{array}$ \\
\hline $\begin{array}{l}11 \\
12 \\
13 \\
14 \\
15\end{array}$ & $\begin{array}{l}18 \\
17 \\
17 \\
18 \\
18\end{array}$ & $\begin{array}{l}15 \\
14 \\
14 \\
14 \\
14\end{array}$ & $\begin{array}{l}17 \\
16 \\
14 \\
15 \\
16\end{array}$ & $\begin{array}{l}14 \\
13 \\
14 \\
16 \\
17\end{array}$ & $\begin{array}{l}12 \\
13 \\
13 \\
13 \\
13\end{array}$ & $\begin{array}{l}15 \\
14 \\
14 \\
15 \\
16\end{array}$ & $\begin{array}{l}23 \\
29 \\
28 \\
25 \\
25\end{array}$ & $\begin{array}{l}17 \\
16 \\
14 \\
14 \\
14\end{array}$ & $\begin{array}{l}16 \\
14 \\
13 \\
13 \\
13\end{array}$ & $\begin{array}{l}10 \\
10 \\
11 \\
11 \\
11\end{array}$ & $\begin{array}{l}8.9 \\
8.9 \\
8.9 \\
9.2 \\
9.1\end{array}$ & $\begin{array}{l}11 \\
12 \\
10 \\
11 \\
10\end{array}$ \\
\hline $\begin{array}{l}16 \\
17 \\
18 \\
19 \\
20\end{array}$ & $\begin{array}{l}18 \\
18 \\
19 \\
17 \\
16\end{array}$ & $\begin{array}{l}14 \\
13 \\
13 \\
14 \\
13\end{array}$ & $\begin{array}{l}15 \\
11 \\
10 \\
10 \\
12\end{array}$ & $\begin{array}{l}16 \\
13 \\
10 \\
8.0 \\
8.0\end{array}$ & $\begin{array}{l}13 \\
14 \\
14 \\
14 \\
13\end{array}$ & $\begin{array}{l}17 \\
18 \\
18 \\
18 \\
18\end{array}$ & $\begin{array}{l}25 \\
24 \\
23 \\
24 \\
27\end{array}$ & $\begin{array}{l}14 \\
13 \\
15 \\
15 \\
13\end{array}$ & $\begin{array}{l}12 \\
12 \\
12 \\
15 \\
15\end{array}$ & $\begin{array}{l}11 \\
11 \\
11 \\
11 \\
13\end{array}$ & $\begin{array}{c}9.1 \\
8.9 \\
8.8 \\
10 \\
9.6\end{array}$ & $\begin{array}{l}11 \\
10 \\
11 \\
11 \\
11\end{array}$ \\
\hline $\begin{array}{l}21 \\
22 \\
23 \\
24 \\
25\end{array}$ & $\begin{array}{l}16 \\
15 \\
15 \\
15 \\
15\end{array}$ & $\begin{array}{l}13 \\
13 \\
13 \\
13 \\
13\end{array}$ & $\begin{array}{c}14 \\
12 \\
10 \\
9.0 \\
9.0\end{array}$ & $\begin{array}{l}8.5 \\
9.0 \\
11 \\
13 \\
14\end{array}$ & $\begin{array}{l}13 \\
13 \\
12 \\
13 \\
13\end{array}$ & $\begin{array}{l}19 \\
18 \\
16 \\
16 \\
19\end{array}$ & $\begin{array}{l}23 \\
23 \\
22 \\
20 \\
20\end{array}$ & $\begin{array}{l}13 \\
14 \\
15 \\
14 \\
13\end{array}$ & $\begin{array}{l}16 \\
14 \\
12 \\
12 \\
12\end{array}$ & $\begin{array}{l}11 \\
11 \\
10 \\
11 \\
11\end{array}$ & $\begin{array}{l}9.4 \\
9.5 \\
9.2 \\
9.3 \\
9.0\end{array}$ & $\begin{array}{l}11 \\
12 \\
12 \\
12 \\
18\end{array}$ \\
\hline $\begin{array}{l}26 \\
27 \\
28 \\
29 \\
30 \\
31\end{array}$ & $\begin{array}{l}15 \\
15 \\
15 \\
14 \\
14 \\
14\end{array}$ & $\begin{array}{r}13 \\
13 \\
13 \\
14 \\
13 \\
-\ldots\end{array}$ & $\begin{array}{l}11 \\
14 \\
14 \\
11 \\
10 \\
10\end{array}$ & $\begin{array}{l}13 \\
12 \\
10 \\
9.0 \\
7.5 \\
7.5\end{array}$ & $\begin{array}{c}14 \\
12 \\
13 \\
\ldots \\
\ldots \\
\ldots\end{array}$ & $\begin{array}{l}19 \\
18 \\
18 \\
17 \\
17 \\
18\end{array}$ & $\begin{array}{r}19 \\
18 \\
18 \\
18 \\
17 \\
---\end{array}$ & $\begin{array}{l}20 \\
17 \\
15 \\
14 \\
15 \\
14\end{array}$ & $\begin{array}{r}15 \\
13 \\
11 \\
11 \\
13 \\
\cdots\end{array}$ & $\begin{array}{c}9.9 \\
9.6 \\
9.6 \\
9.8 \\
11 \\
10\end{array}$ & $\begin{array}{l}9.3 \\
10 \\
10 \\
11 \\
11 \\
9.9\end{array}$ & $\begin{array}{c}14 \\
14 \\
14 \\
13 \\
12 \\
-.-\end{array}$ \\
\hline $\begin{array}{l}\text { TOTAL } \\
\text { MEAN } \\
\text { MAX } \\
\text { MIN } \\
\text { AC-FT }\end{array}$ & $\begin{array}{r}513 \\
16.5 \\
22 \\
14 \\
1020\end{array}$ & $\begin{array}{r}409 \\
13.6 \\
15 \\
13 \\
811\end{array}$ & $\begin{array}{r}396.0 \\
12.8 \\
18 \\
9.0 \\
785\end{array}$ & $\begin{array}{r}403.5 \\
13.0 \\
20 \\
7.5 \\
800\end{array}$ & $\begin{array}{r}336.0 \\
12.0 \\
14 \\
8.0 \\
666\end{array}$ & $\begin{array}{r}495 \\
16.0 \\
19 \\
12 \\
982\end{array}$ & $\begin{array}{r}666 \\
22.2 \\
29 \\
17 \\
1320\end{array}$ & $\begin{array}{r}466 \\
15.0 \\
20 \\
13 \\
924\end{array}$ & $\begin{array}{r}401 \\
13.4 \\
17 \\
11 \\
795\end{array}$ & $\begin{array}{r}327.2 \\
10.6 \\
13 \\
9.1 \\
649\end{array}$ & $\begin{array}{r}292.8 \\
9.45 \\
11 \\
8.8 \\
581\end{array}$ & $\begin{array}{r}343.5 \\
11.4 \\
18 \\
9.2 \\
681\end{array}$ \\
\hline
\end{tabular}

CAL YR 1984 TOTAL 12972.0 MEAN 35.4 MAX 266 MIN 9.0 AC-FT 25730

WTR YR 1985 TOTAL 5049.0 MEAN 13.8 MAX 29 MIN 7.5 AC-FT 10010 
Table 30.--Daily mean discharge, in cubic feet per second, for Whitewood Creek above Whitewood, S. Dak.--Cont inued

DISCHARGE, CUBIC FEET PER SECOND, WATER YEAR OCTOBER 1985 TO SEPTEMBER 1986 MEAN VALUES

\begin{tabular}{|c|c|c|c|c|c|c|c|c|c|c|c|c|}
\hline DAY & OCT & NOV & DEC & JAN & FEB & MAR & APR & MAY & JUN & JUL & AUG & SEP \\
\hline $\begin{array}{l}1 \\
2 \\
3 \\
4 \\
5\end{array}$ & $\begin{array}{l}12 \\
13 \\
15 \\
13 \\
13\end{array}$ & $\begin{array}{l}13 \\
13 \\
13 \\
14 \\
14\end{array}$ & $\begin{array}{r}5.0 \\
7.0 \\
8.5 \\
11 \\
12\end{array}$ & $\begin{array}{l}9.5 \\
9.5 \\
9.0 \\
8.5 \\
8.0\end{array}$ & $\begin{array}{l}16 \\
15 \\
15 \\
15 \\
15\end{array}$ & $\begin{array}{l}24 \\
26 \\
24 \\
24 \\
26\end{array}$ & $\begin{array}{l}26 \\
26 \\
36 \\
31 \\
33\end{array}$ & $\begin{array}{l}77 \\
73 \\
67 \\
64 \\
66\end{array}$ & $\begin{array}{l}25 \\
25 \\
27 \\
39 \\
27\end{array}$ & $\begin{array}{l}25 \\
20 \\
18 \\
17 \\
16\end{array}$ & $\begin{array}{l}20 \\
19 \\
19 \\
19 \\
19\end{array}$ & $\begin{array}{l}15 \\
18 \\
17 \\
16 \\
20\end{array}$ \\
\hline $\begin{array}{r}6 \\
7 \\
8 \\
9 \\
10\end{array}$ & $\begin{array}{l}13 \\
13 \\
13 \\
13 \\
13\end{array}$ & $\begin{array}{l}14 \\
14 \\
13 \\
12 \\
11\end{array}$ & $\begin{array}{l}9.0 \\
8.0 \\
7.0 \\
7.0 \\
6.5\end{array}$ & $\begin{array}{r}8.0 \\
8.5 \\
9.5 \\
11 \\
12\end{array}$ & $\begin{array}{c}14 \\
12 \\
10 \\
9.0 \\
8.0\end{array}$ & $\begin{array}{l}24 \\
22 \\
23 \\
26 \\
26\end{array}$ & $\begin{array}{l}47 \\
61 \\
67 \\
65 \\
63\end{array}$ & $\begin{array}{l}57 \\
54 \\
55 \\
80 \\
72\end{array}$ & $\begin{array}{r}25 \\
24 \\
23 \\
39 \\
130\end{array}$ & $\begin{array}{l}15 \\
14 \\
13 \\
13 \\
14\end{array}$ & $\begin{array}{l}18 \\
17 \\
18 \\
18 \\
18\end{array}$ & $\begin{array}{l}21 \\
17 \\
16 \\
16 \\
22\end{array}$ \\
\hline $\begin{array}{l}11 \\
12 \\
13 \\
14 \\
15\end{array}$ & $\begin{array}{l}13 \\
14 \\
14 \\
14 \\
13\end{array}$ & $\begin{array}{c}10 \\
9.0 \\
8.5 \\
10 \\
13\end{array}$ & $\begin{array}{l}7.5 \\
8.0 \\
9.5 \\
9.0 \\
9.0\end{array}$ & $\begin{array}{l}12 \\
13 \\
12 \\
11 \\
12\end{array}$ & $\begin{array}{r}7.0 \\
6.0 \\
7.5 \\
9.0 \\
10\end{array}$ & $\begin{array}{l}24 \\
24 \\
22 \\
21 \\
21\end{array}$ & $\begin{array}{l}58 \\
50 \\
47 \\
41 \\
40\end{array}$ & $\begin{array}{l}70 \\
67 \\
66 \\
61 \\
56\end{array}$ & $\begin{array}{r}105 \\
79 \\
64 \\
54 \\
46\end{array}$ & $\begin{array}{c}14 \\
13 \\
11 \\
10 \\
9.5\end{array}$ & $\begin{array}{l}18 \\
18 \\
17 \\
16 \\
15\end{array}$ & $\begin{array}{l}19 \\
17 \\
16 \\
16 \\
15\end{array}$ \\
\hline $\begin{array}{l}16 \\
17 \\
18 \\
19 \\
20\end{array}$ & $\begin{array}{l}13 \\
13 \\
11 \\
12 \\
12\end{array}$ & $\begin{array}{l}12 \\
10 \\
9.0 \\
7.5 \\
7.0\end{array}$ & $\begin{array}{l}9.0 \\
9.0 \\
9.5 \\
9.5 \\
9.5\end{array}$ & $\begin{array}{l}13 \\
13 \\
13 \\
15 \\
15\end{array}$ & $\begin{array}{l}12 \\
14 \\
14 \\
14 \\
15\end{array}$ & $\begin{array}{l}20 \\
20 \\
19 \\
18 \\
18\end{array}$ & $\begin{array}{l}38 \\
41 \\
46 \\
45 \\
52\end{array}$ & $\begin{array}{l}52 \\
49 \\
45 \\
43 \\
41\end{array}$ & $\begin{array}{l}39 \\
35 \\
31 \\
30 \\
28\end{array}$ & $\begin{array}{c}9.0 \\
8.1 \\
17 \\
18 \\
18\end{array}$ & $\begin{array}{l}15 \\
15 \\
15 \\
14 \\
14\end{array}$ & $\begin{array}{l}15 \\
14 \\
15 \\
18 \\
15\end{array}$ \\
\hline $\begin{array}{l}21 \\
22 \\
23 \\
24 \\
25\end{array}$ & $\begin{array}{l}12 \\
13 \\
14 \\
13 \\
13\end{array}$ & $\begin{array}{l}7.0 \\
7.0 \\
7.0 \\
7.0 \\
7.5\end{array}$ & $\begin{array}{l}9.5 \\
9.5 \\
9.5 \\
9.5 \\
9.0\end{array}$ & $\begin{array}{l}14 \\
13 \\
12 \\
14 \\
14\end{array}$ & $\begin{array}{l}16 \\
16 \\
17 \\
20 \\
25\end{array}$ & $\begin{array}{l}19 \\
20 \\
19 \\
19 \\
22\end{array}$ & $\begin{array}{r}65 \\
108 \\
152 \\
135 \\
150\end{array}$ & $\begin{array}{l}38 \\
38 \\
38 \\
37 \\
33\end{array}$ & $\begin{array}{l}26 \\
25 \\
23 \\
22 \\
21\end{array}$ & $\begin{array}{l}22 \\
19 \\
19 \\
21 \\
24\end{array}$ & $\begin{array}{l}14 \\
15 \\
15 \\
14 \\
14\end{array}$ & $\begin{array}{l}15 \\
14 \\
14 \\
15 \\
25\end{array}$ \\
\hline $\begin{array}{l}26 \\
27 \\
28 \\
29 \\
30 \\
31\end{array}$ & $\begin{array}{l}13 \\
13 \\
13 \\
13 \\
13 \\
14\end{array}$ & $\begin{array}{l}7.5 \\
7.0 \\
6.5 \\
6.0 \\
6.0 \\
-. .\end{array}$ & $\begin{array}{c}9.0 \\
9.0 \\
9.5 \\
9.5 \\
10 \\
9.5\end{array}$ & $\begin{array}{l}12 \\
11 \\
11 \\
12 \\
14 \\
14\end{array}$ & $\begin{array}{c}29 \\
25 \\
22 \\
\ldots \\
\ldots \\
\ldots\end{array}$ & $\begin{array}{l}21 \\
20 \\
23 \\
24 \\
26 \\
27\end{array}$ & $\begin{array}{r}136 \\
121 \\
109 \\
99 \\
89 \\
\cdots\end{array}$ & $\begin{array}{l}32 \\
30 \\
30 \\
29 \\
28 \\
27\end{array}$ & $\begin{array}{r}22 \\
23 \\
22 \\
21 \\
31 \\
---\end{array}$ & $\begin{array}{l}25 \\
23 \\
20 \\
20 \\
19 \\
20\end{array}$ & $\begin{array}{l}15 \\
15 \\
15 \\
15 \\
15 \\
15\end{array}$ & $\begin{array}{c}16 \\
15 \\
14 \\
14 \\
14 \\
\cdots\end{array}$ \\
\hline $\begin{array}{l}\text { TOTAL } \\
\text { MEAN } \\
\text { MAX } \\
\text { MIN } \\
\text { AC-FT }\end{array}$ & $\begin{array}{r}404 \\
13.0 \\
15 \\
11 \\
801\end{array}$ & $\begin{array}{r}295.5 \\
9.85 \\
14 \\
6.0 \\
586\end{array}$ & $\begin{array}{r}274.0 \\
8.84 \\
12 \\
5.0 \\
543\end{array}$ & $\begin{array}{r}363.5 \\
11.7 \\
15 \\
8.0 \\
721\end{array}$ & $\begin{array}{r}407.5 \\
14.6 \\
29 \\
6.0 \\
808\end{array}$ & $\begin{array}{r}692 \\
22.3 \\
27 \\
18 \\
1370\end{array}$ & $\begin{array}{r}2077 \\
69.2 \\
152 \\
26 \\
4120\end{array}$ & $\begin{array}{r}1575 \\
50.8 \\
80 \\
27 \\
3120\end{array}$ & $\begin{array}{r}1131 \\
37.7 \\
130 \\
21 \\
2240\end{array}$ & $\begin{array}{r}524.6 \\
16.9 \\
25 \\
8.1 \\
1040\end{array}$ & $\begin{array}{r}504 \\
16.3 \\
20 \\
14 \\
1000\end{array}$ & $\begin{array}{r}494 \\
16.5 \\
25 \\
14 \\
980\end{array}$ \\
\hline
\end{tabular}

CAL YR 1985 TOTAL 4704.5 MEAN 12.9 MAX 29 MIN 5.0 AC-FT 9330 WTR YR 1986 TOTAL 8742.1 MEAN 24.0 MAX 152 MIN 5.0 AC-FT 17340 
Table 31.--Daily mean discharge, in cubic feet per second, for whitewood Creek above Vale, S. Dak.

DISCHARGE, CUBIC FEET PER SECOND, WATER YEAR OCTOBER 1984 TO SEPTEMBER 1985 MEAN VALUES

\begin{tabular}{|c|c|c|c|c|c|c|c|c|c|c|c|c|}
\hline DAY & ОСТ & NOV & DEC & JAN & FEB & MAR & APR & MAY & JUN & JUL & AUG & SEP \\
\hline $\begin{array}{l}1 \\
2 \\
3 \\
4 \\
5\end{array}$ & $\begin{array}{l}13 \\
13 \\
14 \\
15 \\
18\end{array}$ & $\begin{array}{l}16 \\
17 \\
18 \\
15 \\
15\end{array}$ & $\begin{array}{l}17 \\
15 \\
14 \\
14 \\
14\end{array}$ & $\begin{array}{r}6.0 \\
7.0 \\
8.0 \\
9.5 \\
11\end{array}$ & $\begin{array}{l}5.2 \\
5.4 \\
5.6 \\
5.8 \\
6.2\end{array}$ & $\begin{array}{l}17 \\
15 \\
14 \\
13 \\
12\end{array}$ & $\begin{array}{l}20 \\
19 \\
14 \\
19 \\
15\end{array}$ & $\begin{array}{l}16 \\
15 \\
15 \\
15 \\
14\end{array}$ & $\begin{array}{c}11 \\
7.2 \\
19 \\
20 \\
14\end{array}$ & $\begin{array}{l}6.0 \\
3.4 \\
3.0 \\
1.9 \\
4.6\end{array}$ & $\begin{array}{l}1.3 \\
1.2 \\
1.0 \\
1.4 \\
1.3\end{array}$ & $\begin{array}{l}2.9 \\
1.1 \\
1.9 \\
2.3 \\
1.2\end{array}$ \\
\hline $\begin{array}{r}6 \\
7 \\
8 \\
9 \\
10\end{array}$ & $\begin{array}{l}21 \\
18 \\
17 \\
16 \\
16\end{array}$ & $\begin{array}{l}15 \\
16 \\
16 \\
17 \\
18\end{array}$ & $\begin{array}{l}16 \\
17 \\
17 \\
16 \\
15\end{array}$ & $\begin{array}{l}11 \\
10 \\
10 \\
9.5 \\
8.5\end{array}$ & $\begin{array}{l}7.0 \\
7.5 \\
9.5 \\
9.0 \\
8.0\end{array}$ & $\begin{array}{l}12 \\
13 \\
14 \\
15 \\
17\end{array}$ & $\begin{array}{c}14 \\
9.7 \\
8.4 \\
13 \\
15\end{array}$ & $\begin{array}{l}14 \\
12 \\
11 \\
11 \\
12\end{array}$ & $\begin{array}{c}13 \\
12 \\
6.6 \\
7.7 \\
12\end{array}$ & $\begin{array}{c}3.8 \\
2.6 \\
1.3 \\
.27 \\
1.9\end{array}$ & $\begin{array}{l}2.3 \\
2.5 \\
3.1 \\
2.2 \\
2.8\end{array}$ & $\begin{array}{l}.72 \\
1.2 \\
1.6 \\
3.2 \\
2.3\end{array}$ \\
\hline $\begin{array}{l}11 \\
12 \\
13 \\
14 \\
15\end{array}$ & $\begin{array}{l}15 \\
16 \\
16 \\
16 \\
17\end{array}$ & $\begin{array}{l}19 \\
19 \\
19 \\
20 \\
19\end{array}$ & $\begin{array}{c}11 \\
8.6 \\
8.0 \\
9.0 \\
10\end{array}$ & $\begin{array}{l}8.0 \\
9.5 \\
11 \\
12 \\
12\end{array}$ & $\begin{array}{l}11 \\
13 \\
14 \\
15 \\
16\end{array}$ & $\begin{array}{l}21 \\
25 \\
34 \\
44 \\
40\end{array}$ & $\begin{array}{l}20 \\
24 \\
29 \\
28 \\
26\end{array}$ & $\begin{array}{l}14 \\
17 \\
12 \\
14 \\
15\end{array}$ & $\begin{array}{c}15 \\
10 \\
9.3 \\
7.9 \\
8.1\end{array}$ & $\begin{array}{c}1.1 \\
.49 \\
.51 \\
2.7 \\
3.6\end{array}$ & $\begin{array}{l}2.3 \\
2.0 \\
2.0 \\
2.6 \\
1.6\end{array}$ & $\begin{array}{l}2.8 \\
3.2 \\
2.5 \\
2.2 \\
3.4\end{array}$ \\
\hline $\begin{array}{l}16 \\
17 \\
18 \\
19 \\
20\end{array}$ & $\begin{array}{l}19 \\
19 \\
20 \\
21 \\
18\end{array}$ & $\begin{array}{l}20 \\
17 \\
17 \\
17 \\
16\end{array}$ & $\begin{array}{l}8.0 \\
8.0 \\
8.4 \\
9.0 \\
9.4\end{array}$ & $\begin{array}{l}12 \\
11 \\
10 \\
9.5 \\
9.0\end{array}$ & $\begin{array}{l}15 \\
15 \\
15 \\
15 \\
16\end{array}$ & $\begin{array}{l}30 \\
24 \\
25 \\
24 \\
22\end{array}$ & $\begin{array}{l}27 \\
26 \\
24 \\
24 \\
28\end{array}$ & $\begin{array}{l}10 \\
11 \\
9.5 \\
13 \\
12\end{array}$ & $\begin{array}{l}6.2 \\
4.0 \\
4.9 \\
8.1 \\
9.8\end{array}$ & $\begin{array}{l}2.9 \\
1.4 \\
1.1 \\
2.1 \\
3.1\end{array}$ & $\begin{array}{l}.81 \\
.33 \\
.08 \\
.00 \\
.89\end{array}$ & $\begin{array}{l}3.4 \\
3.1 \\
3.0 \\
3.3 \\
6.3\end{array}$ \\
\hline $\begin{array}{l}21 \\
22 \\
23 \\
24 \\
25\end{array}$ & $\begin{array}{l}18 \\
18 \\
17 \\
16 \\
17\end{array}$ & $\begin{array}{l}17 \\
18 \\
19 \\
18 \\
16\end{array}$ & $\begin{array}{l}9.6 \\
9.0 \\
8.0 \\
6.0 \\
6.5\end{array}$ & $\begin{array}{l}9.8 \\
11 \\
12 \\
15 \\
14\end{array}$ & $\begin{array}{l}15 \\
15 \\
15 \\
15 \\
14\end{array}$ & $\begin{array}{l}22 \\
22 \\
19 \\
19 \\
20\end{array}$ & $\begin{array}{l}27 \\
27 \\
25 \\
23 \\
25\end{array}$ & $\begin{array}{l}8.4 \\
11 \\
16 \\
12 \\
8.6\end{array}$ & $\begin{array}{l}11 \\
11 \\
5.8 \\
3.0 \\
1.7\end{array}$ & $\begin{array}{l}.00 \\
.00 \\
1.4 \\
2.0 \\
2.7\end{array}$ & $\begin{array}{l}1.8 \\
2.7 \\
2.9 \\
.93 \\
.03\end{array}$ & $\begin{array}{r}5.5 \\
5.7 \\
6.4 \\
8.5 \\
11\end{array}$ \\
\hline $\begin{array}{l}26 \\
27 \\
28 \\
29 \\
30 \\
31\end{array}$ & $\begin{array}{l}15 \\
14 \\
16 \\
14 \\
14 \\
15\end{array}$ & $\begin{array}{r}12 \\
10 \\
16 \\
20 \\
19 \\
---\end{array}$ & $\begin{array}{l}7.5 \\
9.0 \\
7.6 \\
6.6 \\
6.0 \\
5.5\end{array}$ & $\begin{array}{c}13 \\
11 \\
8.0 \\
7.0 \\
6.0 \\
5.0\end{array}$ & $\begin{array}{l}14 \\
16 \\
17 \\
-.- \\
-- \\
-.-\end{array}$ & $\begin{array}{l}21 \\
21 \\
22 \\
20 \\
19 \\
20\end{array}$ & $\begin{array}{c}22 \\
21 \\
20 \\
19 \\
17 \\
\ldots\end{array}$ & $\begin{array}{l}28 \\
55 \\
34 \\
23 \\
12 \\
22\end{array}$ & $\begin{array}{l}4.2 \\
5.8 \\
2.3 \\
3.4 \\
5.1 \\
-. .\end{array}$ & $\begin{array}{l}2.4 \\
1.7 \\
1.5 \\
1.4 \\
2.7 \\
2.0\end{array}$ & $\begin{array}{l}.31 \\
2.3 \\
3.1 \\
3.0 \\
3.3 \\
2.8\end{array}$ & $\begin{array}{c}12 \\
9.0 \\
11 \\
11 \\
11 \\
\ldots\end{array}$ \\
\hline $\begin{array}{l}\text { TOTAL } \\
\text { MEAN } \\
\text { MAX } \\
\text { MIN } \\
\text { AC-FT }\end{array}$ & $\begin{array}{r}512 \\
16.5 \\
21 \\
13 \\
1020\end{array}$ & $\begin{array}{r}511 \\
17.0 \\
20 \\
10 \\
1010\end{array}$ & $\begin{array}{r}325.7 \\
10.5 \\
17 \\
5.5 \\
646\end{array}$ & $\begin{array}{r}306.3 \\
9.88 \\
15 \\
5.0 \\
608\end{array}$ & $\begin{array}{r}335.2 \\
12.0 \\
17 \\
5.2 \\
665\end{array}$ & $\begin{array}{r}656 \\
21.2 \\
44 \\
12 \\
1300\end{array}$ & $\begin{array}{r}629.1 \\
21.0 \\
29 \\
8.4 \\
1250\end{array}$ & $\begin{array}{r}492.5 \\
15.9 \\
55 \\
8.4 \\
977\end{array}$ & $\begin{array}{r}259.1 \\
8.64 \\
20 \\
1.7 \\
514\end{array}$ & $\begin{array}{r}65.57 \\
2.12 \\
6.0 \\
.00 \\
130\end{array}$ & $\begin{array}{r}54.88 \\
1.77 \\
3.3 \\
.00 \\
109\end{array}$ & $\begin{array}{r}142.72 \\
4.76 \\
12 \\
.72 \\
283\end{array}$ \\
\hline
\end{tabular}

CAL YR 1984 TOTAL 16211.2 MEAN 44.3 MAX 604 MIN 5.5 AC-FT 32150

WTR YR 1985 TOTAL 4290.07 MEAN 11.8 MAX 55 MIN .00 AC-FT 8510 
Table 31.--Daily mean discharge, in cubic feet per second, for Whitewood Creek above Vale, S. Dak.--Continued DISCHARGE, CUBIC FEET PER SECOND, WATER YEAR OCTOBER 1985 TO SEPTEMBER 1986 MEAN VALUES

\begin{tabular}{|c|c|c|c|c|c|c|c|c|c|c|c|c|}
\hline DAY & OCT & NOV & DEC & JAN & FEB & MAR & APR & MAY & JUN & JUL & AUG & SEP \\
\hline $\begin{array}{l}1 \\
2 \\
3 \\
4 \\
5\end{array}$ & $\begin{array}{l}11 \\
10 \\
10 \\
13 \\
12\end{array}$ & $\begin{array}{l}11 \\
11 \\
11 \\
12 \\
11\end{array}$ & $\begin{array}{l}8.0 \\
9.0 \\
11 \\
12 \\
10\end{array}$ & $\begin{array}{l}15 \\
14 \\
13 \\
11 \\
10\end{array}$ & $\begin{array}{l}19 \\
20 \\
19 \\
19 \\
19\end{array}$ & $\begin{array}{l}35 \\
35 \\
33 \\
30 \\
30\end{array}$ & $\begin{array}{l}29 \\
29 \\
37 \\
39 \\
58\end{array}$ & $\begin{array}{l}90 \\
86 \\
81 \\
77 \\
79\end{array}$ & $\begin{array}{l}28 \\
27 \\
29 \\
50 \\
35\end{array}$ & $\begin{array}{l}42 \\
26 \\
23 \\
21 \\
21\end{array}$ & $\begin{array}{l}19 \\
17 \\
16 \\
18 \\
15\end{array}$ & $\begin{array}{l}16 \\
17 \\
17 \\
16 \\
17\end{array}$ \\
\hline $\begin{array}{r}6 \\
7 \\
8 \\
9 \\
10\end{array}$ & $\begin{array}{l}11 \\
11 \\
11 \\
12 \\
11\end{array}$ & $\begin{array}{l}11 \\
11 \\
11 \\
11 \\
11\end{array}$ & $\begin{array}{l}10 \\
10 \\
9.0 \\
8.7 \\
9.4\end{array}$ & $\begin{array}{c}9.4 \\
9.0 \\
11 \\
13 \\
15\end{array}$ & $\begin{array}{l}18 \\
17 \\
15 \\
13 \\
12\end{array}$ & $\begin{array}{l}28 \\
31 \\
26 \\
26 \\
28\end{array}$ & $\begin{array}{l}59 \\
62 \\
66 \\
67 \\
67\end{array}$ & $\begin{array}{r}72 \\
67 \\
76 \\
251 \\
103\end{array}$ & $\begin{array}{r}39 \\
37 \\
31 \\
35 \\
124\end{array}$ & $\begin{array}{l}23 \\
25 \\
26 \\
26 \\
25\end{array}$ & $\begin{array}{l}12 \\
12 \\
11 \\
11 \\
10\end{array}$ & $\begin{array}{l}22 \\
19 \\
18 \\
18 \\
22\end{array}$ \\
\hline $\begin{array}{l}11 \\
12 \\
13 \\
14 \\
15\end{array}$ & $\begin{array}{l}11 \\
12 \\
12 \\
12 \\
12\end{array}$ & $\begin{array}{c}10 \\
9.5 \\
9.0 \\
8.7 \\
8.7\end{array}$ & $\begin{array}{l}10 \\
11 \\
12 \\
13 \\
14\end{array}$ & $\begin{array}{l}17 \\
18 \\
17 \\
18 \\
18\end{array}$ & $\begin{array}{c}10 \\
8.0 \\
9.0 \\
11 \\
16\end{array}$ & $\begin{array}{l}27 \\
25 \\
25 \\
23 \\
24\end{array}$ & $\begin{array}{l}63 \\
58 \\
58 \\
66 \\
54\end{array}$ & $\begin{array}{l}90 \\
84 \\
81 \\
75 \\
70\end{array}$ & $\begin{array}{r}119 \\
94 \\
80 \\
72 \\
63\end{array}$ & $\begin{array}{l}23 \\
22 \\
20 \\
18 \\
17\end{array}$ & $\begin{array}{c}8.9 \\
8.4 \\
13 \\
11 \\
9.4\end{array}$ & $\begin{array}{l}23 \\
19 \\
18 \\
18 \\
18\end{array}$ \\
\hline $\begin{array}{l}16 \\
17 \\
18 \\
19 \\
20\end{array}$ & $\begin{array}{c}11 \\
11 \\
10 \\
9.7 \\
10\end{array}$ & $\begin{array}{l}9.5 \\
9.7 \\
9.7 \\
9.7 \\
9.7\end{array}$ & $\begin{array}{l}12 \\
12 \\
12 \\
13 \\
13\end{array}$ & $\begin{array}{l}17 \\
17 \\
14 \\
12 \\
10\end{array}$ & $\begin{array}{l}20 \\
22 \\
23 \\
23 \\
24\end{array}$ & $\begin{array}{l}23 \\
22 \\
23 \\
23 \\
23\end{array}$ & $\begin{array}{r}51 \\
88 \\
176 \\
64 \\
55\end{array}$ & $\begin{array}{l}66 \\
62 \\
55 \\
52 \\
50\end{array}$ & $\begin{array}{l}53 \\
49 \\
42 \\
40 \\
38\end{array}$ & $\begin{array}{l}16 \\
19 \\
22 \\
31 \\
21\end{array}$ & $\begin{array}{l}9.3 \\
8.8 \\
8.6 \\
6.5 \\
6.5\end{array}$ & $\begin{array}{l}18 \\
18 \\
18 \\
21 \\
19\end{array}$ \\
\hline $\begin{array}{l}21 \\
22 \\
23 \\
24 \\
25\end{array}$ & $\begin{array}{l}10 \\
10 \\
11 \\
12 \\
11\end{array}$ & $\begin{array}{l}9.7 \\
9.7 \\
9.7 \\
9.7 \\
9.4\end{array}$ & $\begin{array}{l}13 \\
13 \\
14 \\
13 \\
14\end{array}$ & $\begin{array}{r}8.5 \\
8.0 \\
8.5 \\
9.0 \\
10\end{array}$ & $\begin{array}{l}26 \\
29 \\
32 \\
35 \\
44\end{array}$ & $\begin{array}{l}24 \\
24 \\
24 \\
24 \\
23\end{array}$ & $\begin{array}{r}69 \\
89 \\
153 \\
148 \\
161\end{array}$ & $\begin{array}{l}45 \\
41 \\
43 \\
44 \\
39\end{array}$ & $\begin{array}{l}34 \\
33 \\
30 \\
28 \\
26\end{array}$ & $\begin{array}{l}29 \\
20 \\
19 \\
18 \\
19\end{array}$ & $\begin{array}{l}6.8 \\
8.6 \\
8.2 \\
9.2 \\
9.8\end{array}$ & $\begin{array}{r}19 \\
19 \\
18 \\
390 \\
207\end{array}$ \\
\hline $\begin{array}{l}26 \\
27 \\
28 \\
29 \\
30 \\
31\end{array}$ & $\begin{array}{l}11 \\
10 \\
11 \\
11 \\
10 \\
11\end{array}$ & $\begin{array}{l}9.0 \\
8.8 \\
8.4 \\
8.2 \\
8.0 \\
\ldots .\end{array}$ & $\begin{array}{l}13 \\
13 \\
13 \\
14 \\
14 \\
14\end{array}$ & $\begin{array}{l}11 \\
14 \\
17 \\
18 \\
19 \\
19\end{array}$ & $\begin{array}{c}80 \\
51 \\
37 \\
\ldots \\
\ldots \\
\ldots\end{array}$ & $\begin{array}{l}24 \\
24 \\
24 \\
26 \\
28 \\
28\end{array}$ & $\begin{array}{l}149 \\
169 \\
131 \\
111 \\
102 \\
-\ldots\end{array}$ & $\begin{array}{l}37 \\
35 \\
33 \\
31 \\
30 \\
31\end{array}$ & $\begin{array}{c}27 \\
27 \\
26 \\
23 \\
26 \\
\ldots-\end{array}$ & $\begin{array}{l}32 \\
31 \\
23 \\
20 \\
19 \\
20\end{array}$ & $\begin{array}{c}8.8 \\
8.7 \\
8.0 \\
9.7 \\
10 \\
14\end{array}$ & $\begin{array}{r}43 \\
30 \\
25 \\
23 \\
21 \\
\cdots\end{array}$ \\
\hline $\begin{array}{l}\text { TOTAL } \\
\text { MEAN } \\
\text { MAX } \\
\text { MIN } \\
\text { AC-FT }\end{array}$ & $\begin{array}{r}340.7 \\
11.0 \\
13 \\
9.7 \\
676\end{array}$ & $\begin{array}{r}295.8 \\
9.86 \\
12 \\
8.0 \\
587\end{array}$ & $\begin{array}{r}367.1 \\
11.8 \\
14 \\
8.0 \\
728\end{array}$ & $\begin{array}{r}420.4 \\
13.6 \\
19 \\
8.0 \\
834\end{array}$ & $\begin{array}{r}671.0 \\
24.0 \\
80 \\
8.0 \\
1330\end{array}$ & $\begin{array}{r}813 \\
26.2 \\
35 \\
22 \\
1610\end{array}$ & $\begin{array}{r}2528 \\
84.3 \\
176 \\
29 \\
5010\end{array}$ & $\begin{array}{r}2076 \\
67.0 \\
251 \\
30 \\
4120\end{array}$ & $\begin{array}{r}1365 \\
45.5 \\
124 \\
23 \\
2710\end{array}$ & $\begin{array}{r}717 \\
23.1 \\
42 \\
16 \\
1420\end{array}$ & $\begin{array}{r}333.2 \\
10.7 \\
19 \\
6.5 \\
661\end{array}$ & $\begin{array}{r}1167 \\
38.9 \\
390 \\
16 \\
2310\end{array}$ \\
\hline
\end{tabular}

CAL YR 1985 TOTAL 3944.97 MEAN 10.8 MAX 55 MIN .00 AC-FT 7820

WTR YR 1986 TOTAL 11094.2 MEAN 30.4 MAX 390 MIN 6.5 AC-FT 22010 
Table 32.--Daily mean discharge, in cubic feet per second, for Belle Fourche River near Sturgis, S. Dak.

DISCHARGE, CUBIC FEET PER SECOND, WATER YEAR OCTOBER 1984 TO SEPTEMBER 1985 MEAN VALUES

\begin{tabular}{|c|c|c|c|c|c|c|c|c|c|c|c|}
\hline DAY & CCT & NOV & DEC & JAN & FEB & MAR & APR & MAY & JUN & JUL & AUG \\
\hline $\begin{array}{l}1 \\
2 \\
3 \\
4 \\
5\end{array}$ & $\begin{array}{r}191 \\
140 \\
113 \\
93 \\
82\end{array}$ & $\begin{array}{l}54 \\
52 \\
52 \\
50 \\
50\end{array}$ & $\begin{array}{l}58 \\
47 \\
40 \\
37 \\
37\end{array}$ & $\begin{array}{l}24 \\
26 \\
30 \\
33 \\
35\end{array}$ & $\begin{array}{l}20 \\
21 \\
22 \\
24 \\
26\end{array}$ & $\begin{array}{l}150 \\
140 \\
130 \\
110 \\
100\end{array}$ & $\begin{array}{l}83 \\
81 \\
80 \\
83 \\
83\end{array}$ & $\begin{array}{l}39 \\
38 \\
36 \\
35 \\
67\end{array}$ & $\begin{array}{l}385 \\
350 \\
345 \\
351 \\
296\end{array}$ & $\begin{array}{l}261 \\
240 \\
216 \\
203 \\
219\end{array}$ & $\begin{array}{l}403 \\
403 \\
359 \\
312 \\
279\end{array}$ \\
\hline $\begin{array}{r}6 \\
7 \\
8 \\
9 \\
10\end{array}$ & $\begin{array}{l}84 \\
91 \\
84 \\
77 \\
73\end{array}$ & $\begin{array}{l}50 \\
49 \\
48 \\
48 \\
50\end{array}$ & $\begin{array}{l}38 \\
41 \\
44 \\
46 \\
43\end{array}$ & $\begin{array}{l}34 \\
31 \\
29 \\
28 \\
26\end{array}$ & $\begin{array}{l}27 \\
28 \\
29 \\
28 \\
25\end{array}$ & $\begin{array}{l}180 \\
200 \\
220 \\
250 \\
270\end{array}$ & $\begin{array}{l}76 \\
67 \\
63 \\
59 \\
56\end{array}$ & $\begin{array}{l}57 \\
53 \\
44 \\
51 \\
61\end{array}$ & $\begin{array}{l}259 \\
226 \\
204 \\
166 \\
134\end{array}$ & $\begin{array}{l}216 \\
215 \\
243 \\
263 \\
244\end{array}$ & $\begin{array}{l}257 \\
269 \\
244 \\
242 \\
224\end{array}$ \\
\hline $\begin{array}{l}11 \\
12 \\
13 \\
14 \\
15\end{array}$ & $\begin{array}{l}68 \\
67 \\
65 \\
63 \\
59\end{array}$ & $\begin{array}{l}52 \\
52 \\
52 \\
54 \\
56\end{array}$ & $\begin{array}{l}40 \\
35 \\
30 \\
28 \\
26\end{array}$ & $\begin{array}{l}24 \\
25 \\
26 \\
28 \\
28\end{array}$ & $\begin{array}{l}26 \\
28 \\
30 \\
31 \\
33\end{array}$ & $\begin{array}{l}300 \\
320 \\
350 \\
370 \\
450\end{array}$ & $\begin{array}{l}55 \\
53 \\
51 \\
55 \\
55\end{array}$ & $\begin{array}{l}101 \\
164 \\
163 \\
155 \\
169\end{array}$ & $\begin{array}{l}162 \\
183 \\
181 \\
161 \\
148\end{array}$ & $\begin{array}{l}225 \\
273 \\
309 \\
328 \\
348\end{array}$ & $\begin{array}{l}228 \\
244 \\
236 \\
221 \\
236\end{array}$ \\
\hline $\begin{array}{l}16 \\
17 \\
18 \\
19 \\
20\end{array}$ & $\begin{array}{l}58 \\
58 \\
57 \\
59 \\
62\end{array}$ & $\begin{array}{l}56 \\
56 \\
54 \\
52 \\
49\end{array}$ & $\begin{array}{l}25 \\
24 \\
24 \\
26 \\
32\end{array}$ & $\begin{array}{l}29 \\
31 \\
28 \\
26 \\
24\end{array}$ & $\begin{array}{l}37 \\
41 \\
45 \\
55 \\
70\end{array}$ & $\begin{array}{r}540 \\
700 \\
1000 \\
1500 \\
2340\end{array}$ & $\begin{array}{l}52 \\
50 \\
50 \\
50 \\
49\end{array}$ & $\begin{array}{l}139 \\
147 \\
170 \\
197 \\
224\end{array}$ & $\begin{array}{l}145 \\
150 \\
127 \\
103 \\
104\end{array}$ & $\begin{array}{l}358 \\
351 \\
376 \\
368 \\
357\end{array}$ & $\begin{array}{l}250 \\
280 \\
269 \\
262 \\
263\end{array}$ \\
\hline $\begin{array}{l}21 \\
22 \\
23 \\
24 \\
25\end{array}$ & $\begin{array}{l}60 \\
57 \\
57 \\
57 \\
57\end{array}$ & $\begin{array}{l}47 \\
51 \\
52 \\
58 \\
47\end{array}$ & $\begin{array}{l}32 \\
29 \\
26 \\
23 \\
25\end{array}$ & $\begin{array}{l}24 \\
25 \\
26 \\
28 \\
28\end{array}$ & $\begin{array}{r}80 \\
90 \\
100 \\
110 \\
110\end{array}$ & $\begin{array}{r}1220 \\
747 \\
454 \\
306 \\
244\end{array}$ & $\begin{array}{l}49 \\
53 \\
53 \\
51 \\
46\end{array}$ & $\begin{array}{l}243 \\
241 \\
248 \\
240 \\
256\end{array}$ & $\begin{array}{l}140 \\
163 \\
173 \\
187 \\
195\end{array}$ & $\begin{array}{l}344 \\
336 \\
317 \\
299 \\
252\end{array}$ & $\begin{array}{l}253 \\
281 \\
334 \\
338 \\
347\end{array}$ \\
\hline $\begin{array}{l}26 \\
27 \\
28 \\
29 \\
30 \\
31\end{array}$ & $\begin{array}{l}57 \\
58 \\
57 \\
52 \\
52 \\
54\end{array}$ & $\begin{array}{r}54 \\
19 \\
58 \\
85 \\
75 \\
-\ldots\end{array}$ & $\begin{array}{l}28 \\
35 \\
35 \\
30 \\
26 \\
24\end{array}$ & $\begin{array}{l}28 \\
27 \\
26 \\
25 \\
23 \\
18\end{array}$ & $\begin{array}{l}100 \\
140 \\
160 \\
\ldots . \\
\ldots \\
\ldots\end{array}$ & $\begin{array}{r}197 \\
158 \\
140 \\
109 \\
93 \\
83\end{array}$ & $\begin{array}{r}44 \\
44 \\
44 \\
44 \\
41 \\
-.-\end{array}$ & $\begin{array}{l}300 \\
510 \\
526 \\
482 \\
408 \\
384\end{array}$ & $\begin{array}{l}180 \\
193 \\
225 \\
238 \\
247 \\
\ldots\end{array}$ & $\begin{array}{l}256 \\
256 \\
252 \\
273 \\
303 \\
380\end{array}$ & $\begin{array}{l}371 \\
358 \\
340 \\
322 \\
338 \\
345\end{array}$ \\
\hline $\begin{array}{l}\text { TOTAL } \\
\text { MEAN } \\
\text { MAX } \\
\text { MIN } \\
\text { AC-FT }\end{array}$ & $\begin{array}{r}2262 \\
73.0 \\
191 \\
52 \\
4490\end{array}$ & $\begin{array}{r}1582 \\
52.7 \\
85 \\
19 \\
3140\end{array}$ & $\begin{array}{r}1034 \\
33.4 \\
58 \\
23 \\
2050\end{array}$ & $\begin{array}{r}843 \\
27.2 \\
35 \\
18 \\
1670\end{array}$ & $\begin{array}{r}1536 \\
54.9 \\
160 \\
20 \\
3050\end{array}$ & $\begin{array}{r}13371 \\
431 \\
2340 \\
83 \\
26520\end{array}$ & $\begin{array}{r}1720 \\
57.3 \\
83 \\
41 \\
3410\end{array}$ & $\begin{array}{r}5948 \\
192 \\
526 \\
35 \\
11800\end{array}$ & $\begin{array}{r}6121 \\
204 \\
385 \\
103 \\
12140\end{array}$ & $\begin{array}{r}8881 \\
286 \\
380 \\
203 \\
17620\end{array}$ & $\begin{array}{r}9108 \\
294 \\
403 \\
221 \\
18070\end{array}$ \\
\hline
\end{tabular}

CAL YR 1984 TOTAL 141880 MEAN 388 MAX 5910 MIN 11 AC-FT 281400 WTR YR 1985 TOTAL 57287 MEAN 157 MAX 2340 MIN 18 AC-FT 113600 
Table 32.--Daily mean discharge, in cubic feet per second, for Belle Fourche River near sturgis, S. Dak.--Cont inued

DISCHARGE, CUBIC FEET PER SECOND, WATER YEAR OCTOBER 1985 TO SEPTEMBER 1986 MEAN VALUES

\begin{tabular}{|c|c|c|c|c|c|c|c|c|c|c|c|c|}
\hline DAY & OCT & NOV & DEC & JAN & FEB & MAR & APR & MAY & JUN & JUL & AUG & SEP \\
\hline $\begin{array}{l}1 \\
2 \\
3 \\
4 \\
5\end{array}$ & $\begin{array}{l}56 \\
59 \\
58 \\
55 \\
53\end{array}$ & $\begin{array}{l}46 \\
46 \\
46 \\
46 \\
47\end{array}$ & $\begin{array}{l}26 \\
30 \\
37 \\
46 \\
47\end{array}$ & $\begin{array}{l}46 \\
45 \\
42 \\
40 \\
37\end{array}$ & $\begin{array}{l}56 \\
54 \\
52 \\
49 \\
47\end{array}$ & $\begin{array}{r}1060 \\
975 \\
1720 \\
1390 \\
1780\end{array}$ & $\begin{array}{r}107 \\
99 \\
96 \\
124 \\
280\end{array}$ & $\begin{array}{l}432 \\
312 \\
253 \\
217 \\
195\end{array}$ & $\begin{array}{r}109 \\
96 \\
76 \\
84 \\
100\end{array}$ & $\begin{array}{l}215 \\
242 \\
254 \\
267 \\
262\end{array}$ & $\begin{array}{l}279 \\
266 \\
264 \\
278 \\
314\end{array}$ & $\begin{array}{l}297 \\
354 \\
413 \\
385 \\
315\end{array}$ \\
\hline $\begin{array}{r}6 \\
7 \\
8 \\
9 \\
10\end{array}$ & $\begin{array}{l}54 \\
53 \\
53 \\
50 \\
51\end{array}$ & $\begin{array}{l}47 \\
47 \\
46 \\
46 \\
45\end{array}$ & $\begin{array}{l}47 \\
47 \\
45 \\
41 \\
39\end{array}$ & $\begin{array}{l}37 \\
39 \\
40 \\
41 \\
43\end{array}$ & $\begin{array}{l}45 \\
43 \\
42 \\
40 \\
40\end{array}$ & $\begin{array}{r}1870 \\
1060 \\
1000 \\
761 \\
791\end{array}$ & $\begin{array}{r}1110 \\
571 \\
525 \\
360 \\
224\end{array}$ & $\begin{array}{r}180 \\
193 \\
223 \\
3970 \\
6520\end{array}$ & $\begin{array}{l}158 \\
283 \\
314 \\
305 \\
334\end{array}$ & $\begin{array}{l}257 \\
254 \\
269 \\
270 \\
292\end{array}$ & $\begin{array}{l}335 \\
300 \\
290 \\
317 \\
360\end{array}$ & $\begin{array}{l}362 \\
365 \\
337 \\
337 \\
317\end{array}$ \\
\hline $\begin{array}{l}11 \\
12 \\
13 \\
14 \\
15\end{array}$ & $\begin{array}{l}53 \\
53 \\
55 \\
55 \\
54\end{array}$ & $\begin{array}{l}46 \\
47 \\
47 \\
47 \\
50\end{array}$ & $\begin{array}{l}38 \\
37 \\
36 \\
36 \\
38\end{array}$ & $\begin{array}{l}43 \\
44 \\
45 \\
45 \\
46\end{array}$ & $\begin{array}{l}42 \\
45 \\
49 \\
53 \\
58\end{array}$ & $\begin{array}{l}782 \\
789 \\
645 \\
546 \\
441\end{array}$ & $\begin{array}{l}177 \\
148 \\
133 \\
182 \\
130\end{array}$ & $\begin{array}{r}3880 \\
1300 \\
590 \\
410 \\
360\end{array}$ & $\begin{array}{l}408 \\
316 \\
262 \\
275 \\
280\end{array}$ & $\begin{array}{l}293 \\
264 \\
276 \\
315 \\
341\end{array}$ & $\begin{array}{l}356 \\
345 \\
326 \\
309 \\
315\end{array}$ & $\begin{array}{l}334 \\
340 \\
276 \\
254 \\
248\end{array}$ \\
\hline $\begin{array}{l}16 \\
17 \\
18 \\
19 \\
20\end{array}$ & $\begin{array}{l}54 \\
53 \\
50 \\
49 \\
49\end{array}$ & $\begin{array}{l}54 \\
54 \\
52 \\
50 \\
49\end{array}$ & $\begin{array}{l}38 \\
36 \\
36 \\
38 \\
41\end{array}$ & $\begin{array}{l}47 \\
48 \\
50 \\
54 \\
52\end{array}$ & $\begin{array}{l}60 \\
56 \\
54 \\
52 \\
54\end{array}$ & $\begin{array}{l}337 \\
280 \\
252 \\
201 \\
148\end{array}$ & $\begin{array}{r}135 \\
197 \\
1530 \\
2230 \\
1030\end{array}$ & $\begin{array}{l}291 \\
237 \\
204 \\
178 \\
162\end{array}$ & $\begin{array}{l}211 \\
196 \\
187 \\
154 \\
175\end{array}$ & $\begin{array}{l}355 \\
343 \\
324 \\
411 \\
480\end{array}$ & $\begin{array}{l}323 \\
311 \\
314 \\
311 \\
278\end{array}$ & $\begin{array}{l}261 \\
258 \\
221 \\
239 \\
238\end{array}$ \\
\hline $\begin{array}{l}21 \\
22 \\
23 \\
24 \\
25\end{array}$ & $\begin{array}{l}48 \\
49 \\
49 \\
47 \\
47\end{array}$ & $\begin{array}{l}47 \\
46 \\
39 \\
32 \\
26\end{array}$ & $\begin{array}{l}44 \\
46 \\
45 \\
41 \\
38\end{array}$ & $\begin{array}{l}52 \\
52 \\
52 \\
54 \\
54\end{array}$ & $\begin{array}{r}60 \\
72 \\
82 \\
130 \\
170\end{array}$ & $\begin{array}{l}256 \\
348 \\
309 \\
324 \\
337\end{array}$ & $\begin{array}{l}687 \\
410 \\
327 \\
326 \\
512\end{array}$ & $\begin{array}{l}145 \\
133 \\
124 \\
123 \\
122\end{array}$ & $\begin{array}{l}196 \\
199 \\
207 \\
193 \\
170\end{array}$ & $\begin{array}{l}431 \\
400 \\
379 \\
387 \\
379\end{array}$ & $\begin{array}{l}228 \\
251 \\
273 \\
286 \\
291\end{array}$ & $\begin{array}{r}222 \\
216 \\
216 \\
201 \\
4520\end{array}$ \\
\hline $\begin{array}{l}26 \\
27 \\
28 \\
29 \\
30 \\
31\end{array}$ & $\begin{array}{l}48 \\
47 \\
48 \\
47 \\
47 \\
46\end{array}$ & $\begin{array}{r}24 \\
23 \\
23 \\
24 \\
25 \\
\ldots\end{array}$ & $\begin{array}{l}40 \\
40 \\
41 \\
42 \\
43 \\
45\end{array}$ & $\begin{array}{l}50 \\
50 \\
51 \\
52 \\
52 \\
52\end{array}$ & $\begin{array}{r}300 \\
800 \\
1530 \\
\ldots- \\
\ldots- \\
\ldots\end{array}$ & $\begin{array}{l}265 \\
201 \\
166 \\
146 \\
130 \\
119\end{array}$ & $\begin{array}{r}791 \\
1580 \\
1470 \\
1270 \\
733 \\
\ldots-\end{array}$ & $\begin{array}{r}114 \\
105 \\
96 \\
89 \\
82 \\
76\end{array}$ & $\begin{array}{l}171 \\
181 \\
181 \\
196 \\
199 \\
\ldots\end{array}$ & $\begin{array}{l}365 \\
352 \\
363 \\
352 \\
276 \\
256\end{array}$ & $\begin{array}{l}339 \\
324 \\
293 \\
306 \\
308 \\
283\end{array}$ & $\begin{array}{r}6490 \\
1940 \\
949 \\
477 \\
316 \\
\ldots\end{array}$ \\
\hline $\begin{array}{l}\text { TOTAL } \\
\text { MEAN } \\
\text { MAX } \\
\text { MIN } \\
\text { AC-FT }\end{array}$ & $\begin{array}{r}1590 \\
51.3 \\
59 \\
46 \\
3150\end{array}$ & $\begin{array}{r}1267 \\
42.2 \\
54 \\
23 \\
2510\end{array}$ & $\begin{array}{r}1244 \\
40.1 \\
47 \\
26 \\
2470\end{array}$ & $\begin{array}{r}1455 \\
46.9 \\
54 \\
37 \\
2890\end{array}$ & $\begin{array}{r}4135 \\
148 \\
1530 \\
40 \\
8200\end{array}$ & $\begin{array}{r}19429 \\
627 \\
1870 \\
119 \\
38540\end{array}$ & $\begin{array}{r}17494 \\
583 \\
2230 \\
96 \\
34700\end{array}$ & $\begin{array}{r}21316 \\
688 \\
6520 \\
76 \\
42280\end{array}$ & $\begin{array}{r}6216 \\
207 \\
408 \\
76 \\
12330\end{array}$ & $\begin{array}{r}9924 \\
320 \\
480 \\
215 \\
19680\end{array}$ & $\begin{array}{r}9373 \\
302 \\
360 \\
228 \\
18590\end{array}$ & $\begin{array}{r}21698 \\
723 \\
6490 \\
201 \\
43040\end{array}$ \\
\hline
\end{tabular}

CAL YR 1985 TOTAL 56510 MEAN 155 MAX 2340 MIN 18 AC-FT 112100 WTR YR 1986 TOTAL 115141 MEAN 315 MAX 6520 MIN 23 AC-FT 228400 
Table 33.--Daily mean discharge, in cubic feet per second, for Cheyenne River at Cherry Creek, S. Dak.

DISCHARGE, CUBIC FEET PER SECOND, WATER YEAR OCTOBER 1984 TO SEPTEMBER 1985 MEAN VALUES

\begin{tabular}{|c|c|c|c|c|c|c|c|c|c|c|c|c|}
\hline DAY & OCT & NOV & DEC & JAN & FEB & MAR & APR & MAY & JUN & JUL & AUG & SEF \\
\hline $\begin{array}{l}1 \\
2 \\
3 \\
4 \\
5\end{array}$ & $\begin{array}{l}412 \\
397 \\
344 \\
287 \\
260\end{array}$ & $\begin{array}{l}247 \\
220 \\
183 \\
204 \\
211\end{array}$ & $\begin{array}{r}130 \\
110 \\
90 \\
90 \\
85\end{array}$ & $\begin{array}{l}130 \\
140 \\
150 \\
160 \\
170\end{array}$ & $\begin{array}{l}80 \\
70 \\
55 \\
55 \\
60\end{array}$ & $\begin{array}{l}230 \\
220 \\
200 \\
200 \\
220\end{array}$ & $\begin{array}{l}482 \\
428 \\
400 \\
381 \\
365\end{array}$ & $\begin{array}{l}214 \\
213 \\
219 \\
190 \\
181\end{array}$ & $\begin{array}{l}443 \\
401 \\
404 \\
412 \\
407\end{array}$ & $\begin{array}{l}261 \\
252 \\
247 \\
266 \\
242\end{array}$ & $\begin{array}{l}522 \\
587 \\
497 \\
421 \\
375\end{array}$ & $\begin{array}{l}27 \\
28\end{array}$ \\
\hline $\begin{array}{r}6 \\
7 \\
8 \\
9 \\
10\end{array}$ & $\begin{array}{l}242 \\
226 \\
217 \\
211 \\
209\end{array}$ & $\begin{array}{l}213 \\
222 \\
215 \\
214 \\
215\end{array}$ & $\begin{array}{r}80 \\
90 \\
120 \\
160 \\
200\end{array}$ & $\begin{array}{l}160 \\
160 \\
150 \\
140 \\
130\end{array}$ & $\begin{array}{l}60 \\
60 \\
62 \\
65 \\
70\end{array}$ & $\begin{array}{l}220 \\
240 \\
250 \\
250 \\
250\end{array}$ & $\begin{array}{l}360 \\
382 \\
356 \\
326 \\
299\end{array}$ & $\begin{array}{l}177 \\
176 \\
162 \\
154 \\
157\end{array}$ & $\begin{array}{l}393 \\
379 \\
322 \\
298 \\
285\end{array}$ & $\begin{array}{l}213 \\
195 \\
207 \\
201 \\
194\end{array}$ & $\begin{array}{l}321 \\
260 \\
243 \\
210 \\
209\end{array}$ & 33 \\
\hline $\begin{array}{l}11 \\
12 \\
13 \\
14 \\
15\end{array}$ & $\begin{array}{l}209 \\
209 \\
204 \\
196 \\
190\end{array}$ & $\begin{array}{l}218 \\
239 \\
244 \\
242 \\
282\end{array}$ & $\begin{array}{l}190 \\
180 \\
180 \\
190 \\
200\end{array}$ & $\begin{array}{l}115 \\
130 \\
140 \\
160 \\
160\end{array}$ & $\begin{array}{r}90 \\
110 \\
120 \\
130 \\
130\end{array}$ & $\begin{array}{l}270 \\
270 \\
280 \\
300 \\
500\end{array}$ & $\begin{array}{l}286 \\
269 \\
262 \\
252 \\
244\end{array}$ & $\begin{array}{l}135 \\
134 \\
131 \\
136 \\
163\end{array}$ & $\begin{array}{l}270 \\
270 \\
330 \\
301 \\
285\end{array}$ & $\begin{array}{l}205 \\
203 \\
202 \\
196 \\
224\end{array}$ & $\begin{array}{l}182 \\
184 \\
152 \\
149 \\
223\end{array}$ & $\begin{array}{l}28 \\
29\end{array}$ \\
\hline $\begin{array}{l}16 \\
17 \\
18 \\
19 \\
20\end{array}$ & $\begin{array}{l}184 \\
184 \\
182 \\
180 \\
178\end{array}$ & $\begin{array}{l}290 \\
242 \\
240 \\
250 \\
250\end{array}$ & $\begin{array}{l}190 \\
180 \\
170 \\
180 \\
190\end{array}$ & $\begin{array}{l}170 \\
180 \\
180 \\
170 \\
150\end{array}$ & $\begin{array}{l}140 \\
140 \\
150 \\
150 \\
160\end{array}$ & $\begin{array}{l}1700 \\
5050 \\
4210 \\
3950 \\
4280\end{array}$ & $\begin{array}{l}238 \\
232 \\
233 \\
234 \\
244\end{array}$ & $\begin{array}{l}212 \\
222 \\
219 \\
206 \\
198\end{array}$ & $\begin{array}{l}252 \\
215 \\
198 \\
185 \\
181\end{array}$ & $\begin{array}{l}252 \\
265 \\
347 \\
480 \\
392\end{array}$ & $\begin{array}{l}243 \\
191 \\
177 \\
172 \\
178\end{array}$ & $\begin{array}{l}30 \\
24\end{array}$ \\
\hline $\begin{array}{l}21 \\
22 \\
23 \\
24 \\
25\end{array}$ & $\begin{array}{l}176 \\
178 \\
183 \\
188 \\
190\end{array}$ & $\begin{array}{l}240 \\
236 \\
219 \\
220 \\
210\end{array}$ & $\begin{array}{l}180 \\
170 \\
170 \\
150 \\
160\end{array}$ & $\begin{array}{l}160 \\
160 \\
180 \\
180 \\
170\end{array}$ & $\begin{array}{l}160 \\
180 \\
200 \\
200 \\
210\end{array}$ & $\begin{array}{l}5200 \\
3300 \\
2500 \\
2100 \\
1750\end{array}$ & $\begin{array}{l}252 \\
253 \\
261 \\
242 \\
237\end{array}$ & $\begin{array}{l}202 \\
214 \\
253 \\
252 \\
239\end{array}$ & $\begin{array}{l}169 \\
166 \\
160 \\
153 \\
151\end{array}$ & $\begin{array}{l}367 \\
341 \\
295 \\
312 \\
303\end{array}$ & $\begin{array}{l}186 \\
686 \\
421 \\
305 \\
281\end{array}$ & $\begin{array}{l}175 \\
176 \\
174 \\
178 \\
18\end{array}$ \\
\hline $\begin{array}{l}26 \\
27 \\
28 \\
29 \\
30 \\
31\end{array}$ & $\begin{array}{l}189 \\
180 \\
178 \\
184 \\
190 \\
196\end{array}$ & $\begin{array}{l}200 \\
200 \\
180 \\
170 \\
160 \\
\cdots\end{array}$ & $\begin{array}{l}160 \\
170 \\
160 \\
150 \\
130 \\
120\end{array}$ & $\begin{array}{l}180 \\
170 \\
150 \\
140 \\
120 \\
100\end{array}$ & $\begin{array}{l}200 \\
200 \\
230 \\
\ldots- \\
\ldots- \\
\ldots-\end{array}$ & $\begin{array}{r}1500 \\
1300 \\
781 \\
681 \\
595 \\
536\end{array}$ & $\begin{array}{l}228 \\
218 \\
211 \\
207 \\
213 \\
\cdots-\end{array}$ & $\begin{array}{l}247 \\
266 \\
289 \\
440 \\
575 \\
529\end{array}$ & $\begin{array}{l}167 \\
178 \\
193 \\
205 \\
210 \\
-\cdots\end{array}$ & $\begin{array}{l}272 \\
247 \\
212 \\
222 \\
231 \\
352\end{array}$ & $\begin{array}{l}297 \\
325 \\
335 \\
288 \\
276 \\
253\end{array}$ & $\begin{array}{l}199 \\
192 \\
187 \\
-\end{array}$ \\
\hline $\begin{array}{l}\text { TOTAL } \\
\text { MEAN } \\
\text { MAX } \\
\text { MIN } \\
\text { AC-FT }\end{array}$ & $\begin{array}{r}6753 \\
218 \\
412 \\
176 \\
13390\end{array}$ & $\begin{array}{r}6676 \\
223 \\
290 \\
160 \\
13240\end{array}$ & $\begin{array}{r}4725 \\
152 \\
200 \\
80 \\
9370\end{array}$ & $\begin{array}{r}4755 \\
153 \\
180 \\
100 \\
9430\end{array}$ & $\begin{array}{r}3537 \\
126 \\
230 \\
55 \\
7020\end{array}$ & $\begin{array}{r}43333 \\
1398 \\
5200 \\
200 \\
85950\end{array}$ & $\begin{array}{r}8595 \\
286 \\
482 \\
207 \\
17050\end{array}$ & $\begin{array}{r}7105 \\
229 \\
575 \\
131 \\
14090\end{array}$ & $\begin{array}{r}7983 \\
266 \\
443 \\
151 \\
15830\end{array}$ & $\begin{array}{r}8198 \\
264 \\
480 \\
194 \\
16260\end{array}$ & $\begin{array}{r}9149 \\
295 \\
686 \\
149 \\
18150\end{array}$ & $\begin{array}{r}8538 \\
285 \\
752 \\
173 \\
16940\end{array}$ \\
\hline
\end{tabular}

CAL YR 1984 TOTAL 387820 MEAN 1060 MAX 17400 MIN 50 AC-FT 769200

WTR YR 1985 TOTAL 119347 MEAN 327 MAX 5200 MIN 55 AC-FT 236700 
Table 33.- -Daily mean discharge, in cubic feet per second, for Cheyenne River at Cherry Creek, S. Dak.--Cont inued

DISCHARGE, CUBIC FEET PER SECOND, WATER YEAR OCTOBER 1985 TO SEPTEMBER 1986 MEAN VALUES

\begin{tabular}{|c|c|c|c|c|c|c|c|c|c|c|c|c|}
\hline DAY & OCT & NoV & DEC & JAN & FEB & MAR & APR & MAY & JUN & JUL & AUG & SEP \\
\hline $\begin{array}{l}1 \\
2 \\
3 \\
4 \\
5\end{array}$ & $\begin{array}{l}184 \\
184 \\
171 \\
169 \\
168\end{array}$ & $\begin{array}{l}156 \\
156 \\
156 \\
161 \\
165\end{array}$ & $\begin{array}{l}80 \\
75 \\
80 \\
80 \\
80\end{array}$ & $\begin{array}{r}100 \\
100 \\
100 \\
95 \\
95\end{array}$ & $\begin{array}{l}250 \\
260 \\
270 \\
270 \\
260\end{array}$ & $\begin{array}{l}11800 \\
10800 \\
10700 \\
10500 \\
10400\end{array}$ & $\begin{array}{r}930 \\
788 \\
681 \\
907 \\
2450\end{array}$ & $\begin{array}{l}5080 \\
2830 \\
1800 \\
1400 \\
1030\end{array}$ & $\begin{array}{l}437 \\
400 \\
400 \\
400 \\
450\end{array}$ & $\begin{array}{l}474 \\
665 \\
907 \\
802 \\
834\end{array}$ & $\begin{array}{l}418 \\
320 \\
320 \\
300 \\
300\end{array}$ & $\begin{array}{l}400 \\
400 \\
380 \\
464 \\
430\end{array}$ \\
\hline $\begin{array}{r}6 \\
7 \\
8 \\
9 \\
10\end{array}$ & $\begin{array}{l}166 \\
158 \\
156 \\
158 \\
160\end{array}$ & $\begin{array}{l}162 \\
162 \\
172 \\
160 \\
150\end{array}$ & $\begin{array}{l}80 \\
85 \\
85 \\
80 \\
80\end{array}$ & $\begin{array}{r}90 \\
90 \\
100 \\
140 \\
200\end{array}$ & $\begin{array}{l}250 \\
240 \\
210 \\
180 \\
160\end{array}$ & $\begin{array}{r}10600 \\
10200 \\
6540 \\
5800 \\
6790\end{array}$ & $\begin{array}{l}5800 \\
8870 \\
5380 \\
3940 \\
2670\end{array}$ & $\begin{array}{r}817 \\
767 \\
1310 \\
8410 \\
21400\end{array}$ & $\begin{array}{l}520 \\
486 \\
448 \\
508 \\
810\end{array}$ & $\begin{array}{l}790 \\
676 \\
550 \\
500 \\
582\end{array}$ & $\begin{array}{l}350 \\
366 \\
350 \\
430 \\
430\end{array}$ & $\begin{array}{l}520 \\
740 \\
560 \\
480 \\
470\end{array}$ \\
\hline $\begin{array}{l}11 \\
12 \\
13 \\
14 \\
15\end{array}$ & $\begin{array}{l}154 \\
163 \\
166 \\
210 \\
236\end{array}$ & $\begin{array}{l}150 \\
160 \\
180 \\
180 \\
180\end{array}$ & $\begin{array}{l}75 \\
75 \\
70 \\
80 \\
90\end{array}$ & $\begin{array}{l}300 \\
320 \\
320 \\
330 \\
330\end{array}$ & $\begin{array}{l}120 \\
110 \\
110 \\
130 \\
130\end{array}$ & $\begin{array}{l}6460 \\
5910 \\
5930 \\
5420 \\
4090\end{array}$ & $\begin{array}{r}1560 \\
1070 \\
877 \\
681 \\
630\end{array}$ & $\begin{array}{r}20900 \\
12200 \\
7300 \\
4980 \\
3740\end{array}$ & $\begin{array}{r}10800 \\
5050 \\
2990 \\
7470 \\
6680\end{array}$ & $\begin{array}{l}700 \\
600 \\
450 \\
420 \\
470\end{array}$ & $\begin{array}{l}400 \\
400 \\
400 \\
422 \\
650\end{array}$ & $\begin{array}{l}500 \\
560 \\
490 \\
380 \\
340\end{array}$ \\
\hline $\begin{array}{l}16 \\
17 \\
18 \\
19 \\
20\end{array}$ & $\begin{array}{l}204 \\
191 \\
173 \\
163 \\
162\end{array}$ & $\begin{array}{l}170 \\
170 \\
180 \\
160 \\
140\end{array}$ & $\begin{array}{r}85 \\
85 \\
80 \\
80 \\
100\end{array}$ & $\begin{array}{l}350 \\
400 \\
400 \\
420 \\
450\end{array}$ & $\begin{array}{l}150 \\
160 \\
150 \\
150 \\
140\end{array}$ & $\begin{array}{l}3080 \\
2280 \\
2220 \\
1780 \\
1410\end{array}$ & $\begin{array}{r}1000 \\
2000 \\
6700 \\
12200 \\
11400\end{array}$ & $\begin{array}{l}3000 \\
3500 \\
2500 \\
2000 \\
1600\end{array}$ & $\begin{array}{l}5320 \\
4370 \\
2800 \\
2100 \\
1540\end{array}$ & $\begin{array}{l}600 \\
550 \\
500 \\
450 \\
500\end{array}$ & $\begin{array}{l}550 \\
450 \\
400 \\
380 \\
345\end{array}$ & $\begin{array}{r}320 \\
340 \\
470 \\
1500 \\
700\end{array}$ \\
\hline $\begin{array}{l}21 \\
22 \\
23 \\
24 \\
25\end{array}$ & $\begin{array}{l}157 \\
151 \\
145 \\
148 \\
154\end{array}$ & $\begin{array}{l}120 \\
120 \\
110 \\
110 \\
120\end{array}$ & $\begin{array}{r}115 \\
110 \\
105 \\
95 \\
95\end{array}$ & $\begin{array}{l}420 \\
400 \\
350 \\
300 \\
270\end{array}$ & $\begin{array}{l}140 \\
140 \\
140 \\
153 \\
300\end{array}$ & $\begin{array}{l}1280 \\
2660 \\
5560 \\
4800 \\
3670\end{array}$ & $\begin{array}{l}6040 \\
4890 \\
3420 \\
2050 \\
1760\end{array}$ & $\begin{array}{r}1300 \\
1200 \\
1100 \\
900 \\
800\end{array}$ & $\begin{array}{r}1180 \\
946 \\
810 \\
676 \\
609\end{array}$ & $\begin{array}{l}469 \\
580 \\
520 \\
550 \\
500\end{array}$ & $\begin{array}{l}357 \\
270 \\
300 \\
330 \\
350\end{array}$ & $\begin{array}{r}550 \\
380 \\
590 \\
450 \\
1000\end{array}$ \\
\hline $\begin{array}{l}26 \\
27 \\
28 \\
29 \\
30 \\
31\end{array}$ & $\begin{array}{l}156 \\
163 \\
164 \\
162 \\
161 \\
159\end{array}$ & $\begin{array}{r}110 \\
90 \\
80 \\
80 \\
80 \\
-.-\end{array}$ & $\begin{array}{r}100 \\
100 \\
95 \\
90 \\
90 \\
95\end{array}$ & $\begin{array}{l}260 \\
250 \\
270 \\
270 \\
270 \\
250\end{array}$ & $\begin{array}{r}800 \\
5000 \\
17600 \\
\ldots- \\
\ldots- \\
\ldots-\end{array}$ & $\begin{array}{l}3200 \\
2570 \\
2000 \\
1580 \\
1330 \\
1070\end{array}$ & $\begin{array}{r}2310 \\
9760 \\
13400 \\
8370 \\
6350 \\
\ldots .-\end{array}$ & $\begin{array}{l}700 \\
650 \\
600 \\
571 \\
500 \\
464\end{array}$ & $\begin{array}{l}571 \\
509 \\
460 \\
450 \\
469 \\
\cdots--\end{array}$ & $\begin{array}{l}450 \\
450 \\
500 \\
550 \\
450 \\
400\end{array}$ & $\begin{array}{l}345 \\
410 \\
383 \\
360 \\
380 \\
360\end{array}$ & $\begin{array}{r}2770 \\
5400 \\
3670 \\
1710 \\
941 \\
\ldots\end{array}$ \\
\hline $\begin{array}{l}\text { TOTAL } \\
\text { MEAN } \\
\text { MAX } \\
\text { MIN } \\
\text { AC-FT }\end{array}$ & $\begin{array}{r}5216 \\
168 \\
236 \\
145 \\
10350\end{array}$ & $\begin{array}{r}4290 \\
143 \\
180 \\
80 \\
8510\end{array}$ & $\begin{array}{r}2715 \\
87.6 \\
115 \\
70 \\
5390\end{array}$ & $\begin{array}{r}8040 \\
259 \\
450 \\
90 \\
15950\end{array}$ & $\begin{array}{r}27973 \\
999 \\
17600 \\
110 \\
55480\end{array}$ & $\begin{array}{r}162430 \\
5240 \\
11800 \\
1070 \\
322200\end{array}$ & $\begin{array}{r}128884 \\
4296 \\
13400 \\
630 \\
255600\end{array}$ & $\begin{array}{r}115349 \\
3721 \\
21400 \\
464 \\
228800\end{array}$ & $\begin{array}{r}60659 \\
2022 \\
10800 \\
400 \\
120300\end{array}$ & $\begin{array}{r}17439 \\
563 \\
907 \\
400 \\
34590\end{array}$ & $\begin{array}{r}11826 \\
381 \\
650 \\
270 \\
23460\end{array}$ & $\begin{array}{r}27905 \\
930 \\
5400 \\
320 \\
55350\end{array}$ \\
\hline
\end{tabular}

CAL YR 1985 TOTAL 113414 MEAN 311 MAX 5200 MIN 55 AC-FT 225000 WTR YR 1986 TOTAL 572726 MEAN 1569 MAX 21400 MIN 70 AC-FT 1136000 


\section{Water-Quality Data}

Water-quality data were obtained at 18 sites during the 1985-86 water years (figs. 1 and 2, table 1). Monthly samples and storm runoff samples were taken at four streamflow gaging stations--Whitewood Creek above Whitewood, Whitewood Creek above Vale, Belle Fourche River near Sturgis, and Cheyenne River at Cherry Creek. One to five samples were collected at each of the other sites.

Samples were collected and analyzed according to procedures published by the U.S. Geological Survey (Guy, 1969; Barnett and Mallory, 1971; Skougstad and others, 1979; Friedman and Erdmann, 1982; Guy and Norman, 1982; Wershaw and others, 1987). Samples for inorganic and suspended-sediment analyses normally were obtained by wading the stream and collecting depth-integrated aliquots with a sampling rod that held two $2-L$ bottles equipped with sampling nozzles. Aliquots were collected at from 10 to 25 equally spaced verticals across the channel and were composited in a U.S. Geological Survey samplesplitting churn. Except for total organic carbon, samples for raw-water analyses were filled from the churn. Bottles for dissolved constituents were filled by pumping the water from the churn with a peristaltic pump and filtering the water through a $0.45-\mu \mathrm{m}$ cellulose filter. Samples to be analyzed for nitrogen species and phosphorus were placed in brown polyethelene bottles and preserved with mercuric-chloride and put on ice. Samples to be analyzed for dissolved trace metals and major cations were preserved with nitric acid. Samples to be analyzed for dissolved mercury were placed in glass bottles and preserved with chromic acid. Samples to be analyzed for cyanide were preserved with $\mathrm{NaOH}$ and put on ice. Samples were then shipped to the U.S. Geological Survey's Central Laboratory in Arvada, Colorado, for analysis. Chemical analysis data are listed in tables 34 through 38.

Suspended-sediment samples were concentrated by centrifugation using a Beckman model with a JA-10 fixed angle rotor. Samples were spun at 5,000 revolutions per minute for 5 minutes. Supernate was removed from the sediment with a peristaltic pump and the sediment was freeze dried, aggregated, powdered, and homogenized before subsampling for total chemical analysis.

Total chemical analyses of subsamples for iron, manganese, aluminum, titanium, arsenic, antimony, cadmium, chromium, cobalt, copper, lead, mercury, nickel, selenium, and zinc were performed by Arthur J. Horowitz and Kent Elrick, U.S. Geological Survey, Doraville, Georgia. The procedures followed were those of Horowitz and Elrick (1985, 1986). Subsamples for all elements except mercury were digested with a combination of $\mathrm{HF} / \mathrm{HClO}_{4} / \mathrm{HNO}_{3}$ acids at $200{ }^{\circ} \mathrm{C}$. Subsamples for mercury were digested with Laforte aqua regia at $100{ }^{\circ} \mathrm{C}$. Quantification was by atomic hydride generation. The procedures followed for total organic carbon analyses were standard U.S. Geological Survey procedures for the analysis of bottom materials as described by Wershaw and others (1987). The result of 56 analyses of suspended sediment from 10 sites are listed in table 39. 
Table 34. -- Chemical analyses of surface-water samples collected at whitewood Creek above Whitewood, S. Dak.

WATER QUALITY DATA

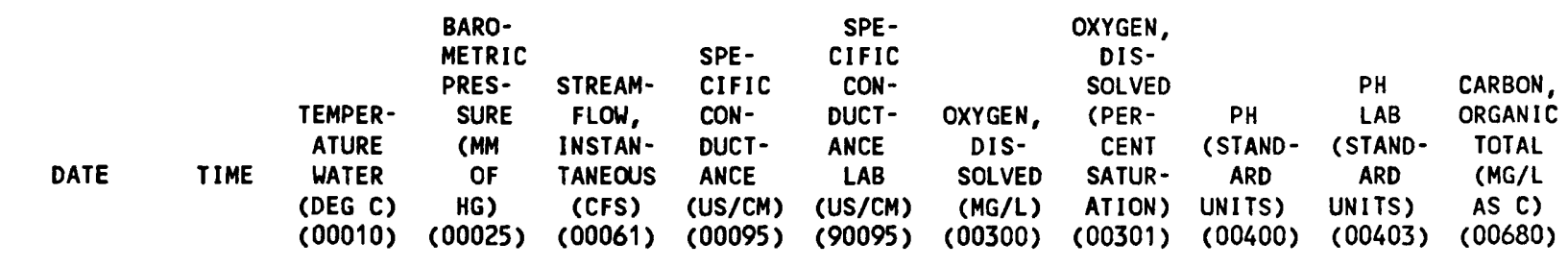

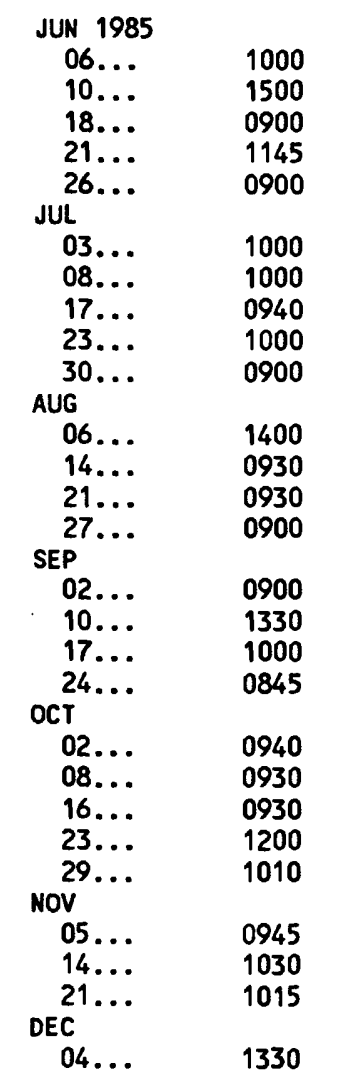

\begin{tabular}{|c|c|c|c|c|c|c|c|c|c|}
\hline $\begin{array}{l}-- \\
--\end{array}$ & $\begin{array}{l}-- \\
--\end{array}$ & $\begin{array}{l}14 \\
20\end{array}$ & 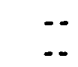 & $\begin{array}{l}916 \\
850\end{array}$ & $=$ & -- & $\ddot{-}$ & $\begin{array}{l}8.40 \\
8.40\end{array}$ & $\begin{array}{l}2.3 \\
3.9\end{array}$ \\
\hline $\begin{array}{l}12.5 \\
14.5 \\
12.0\end{array}$ & $\begin{array}{l}670 \\
748 \\
669\end{array}$ & $\begin{array}{l}12 \\
20 \\
13\end{array}$ & $\begin{array}{l}958 \\
847 \\
929\end{array}$ & $\begin{array}{r}859 \\
940\end{array}$ & $\begin{array}{r}10.1 \\
9.7 \\
9.5\end{array}$ & $\begin{array}{r}109 \\
97 \\
101\end{array}$ & $\begin{array}{l}8.80 \\
8.90 \\
8.80\end{array}$ & $\begin{array}{r}-. \\
8.50 \\
8.40\end{array}$ & $\begin{array}{l}3.9 \\
3.9\end{array}$ \\
\hline $\begin{array}{l}18.5 \\
21.0 \\
20.0 \\
20.5 \\
15.5\end{array}$ & $\begin{array}{l}-. \\
650 \\
670 \\
662 \\
670\end{array}$ & $\begin{array}{c}11 \\
9.1 \\
11 \\
11 \\
11\end{array}$ & $\begin{array}{r}968 \\
931 \\
1010 \\
1070 \\
1020\end{array}$ & $\begin{array}{c}-. \\
923 \\
-- \\
-- \\
--\end{array}$ & $\begin{array}{r}-. \\
9.8 \\
9.2 \\
8.7 \\
--\end{array}$ & $\begin{array}{r}-- \\
130 \\
116 \\
112 \\
--\end{array}$ & $\begin{array}{l}9.20 \\
9.30 \\
9.10 \\
9.10 \\
8.70\end{array}$ & $\begin{array}{r}-. \\
8.80 \\
\ldots \\
- \\
-.\end{array}$ & $\begin{array}{c}- \\
2.4 \\
\cdots \\
- \\
-\end{array}$ \\
\hline $\begin{array}{l}25.0 \\
12.0 \\
19.5 \\
17.5\end{array}$ & $\begin{array}{l}670 \\
671 \\
670 \\
670\end{array}$ & $\begin{array}{l}11 \\
8.6 \\
9.4 \\
9.6\end{array}$ & $\begin{array}{l}1120 \\
1070 \\
1060 \\
1100\end{array}$ & $\begin{array}{c}1090 \\
\ldots \\
- \\
--\end{array}$ & $\begin{array}{l}8.5 \\
9.7 \\
8.1 \\
9.0\end{array}$ & $\begin{array}{l}118 \\
103 \\
101 \\
108\end{array}$ & $\begin{array}{l}9.20 \\
8.80 \\
8.60 \\
8.60\end{array}$ & $\begin{array}{c}8.70 \\
\ldots \\
-- \\
--\end{array}$ & $\begin{array}{r}3.4 \\
\ldots \\
- \\
--\end{array}$ \\
\hline 17.0 & 660 & 10 & 1070 & 1060 & 12.0 & 145 & 8.70 & 8.50 & 3.0 \\
\hline $\begin{array}{r}12.0 \\
3.5\end{array}$ & $\begin{array}{l}- \\
660 \\
660\end{array}$ & $\begin{array}{l}11 \\
10 \\
11\end{array}$ & $\begin{array}{r}1050 \\
917\end{array}$ & $\begin{array}{l}-- \\
\cdots \\
-\end{array}$ & $\begin{array}{r}10.0 \\
11.3\end{array}$ & $\begin{array}{r}\ddot{108} \\
99\end{array}$ & $\begin{array}{r}-- \\
8.50 \\
8.50\end{array}$ & $\begin{array}{l}- \\
\cdots \\
-\end{array}$ & $\begin{array}{l}- \\
- \\
-\end{array}$ \\
\hline $\begin{array}{l}7.5 \\
3.5 \\
6.0 \\
8.0 \\
6.0\end{array}$ & $\begin{array}{l}670 \\
670 \\
660 \\
660 \\
680\end{array}$ & $\begin{array}{l}12 \\
14 \\
13 \\
13 \\
12\end{array}$ & $\begin{array}{r}969 \\
945 \\
1000 \\
948 \\
955\end{array}$ & $\begin{array}{r}-- \\
986 \\
-- \\
-- \\
--\end{array}$ & $\begin{array}{r}10.0 \\
10.6 \\
9.9 \\
10.5 \\
11.7\end{array}$ & $\begin{array}{r}95 \\
91 \\
93 \\
103 \\
106\end{array}$ & $\begin{array}{l}8.30 \\
8.40 \\
8.20 \\
8.50 \\
8.50\end{array}$ & $\begin{array}{c}-. \\
8.40 \\
.- \\
-- \\
.-\end{array}$ & $\begin{array}{c}-- \\
2.4 \\
-- \\
-\end{array}$ \\
\hline $\begin{array}{l}7.5 \\
0.0 \\
0.0\end{array}$ & $\begin{array}{l}666 \\
672 \\
653\end{array}$ & $\begin{array}{l}13 \\
13 \\
71\end{array}$ & $\begin{array}{l}1080 \\
1040 \\
1210\end{array}$ & $\begin{array}{r}1100 \\
\ldots \\
\ldots\end{array}$ & $\begin{array}{l}11.9 \\
11.6 \\
13.1\end{array}$ & $\begin{array}{r}114 \\
91 \\
105\end{array}$ & $\begin{array}{l}8.40 \\
8.11 \\
7.92\end{array}$ & $\begin{array}{c}8.40 \\
\ldots \\
\ldots\end{array}$ & $\begin{array}{r}2.1 \\
- \\
-\end{array}$ \\
\hline 0.0 & 670 & 16 & 1010 & 1050 & 11.6 & 91 & 8.20 & 8.10 & 3.9 \\
\hline
\end{tabular}


Table 34.--Chemical analyses of surface-water samples collected at Whitewood Creek above Whitewood, S. Dak.--Cont inued

WATER QUALITY DATA

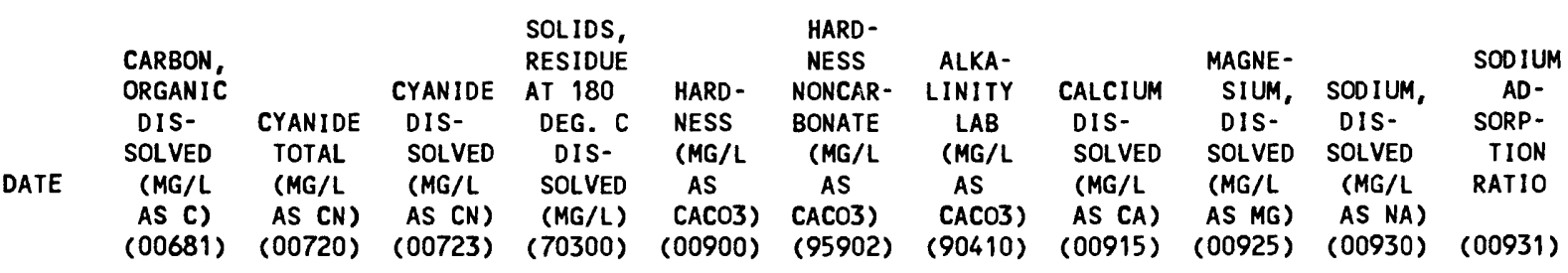

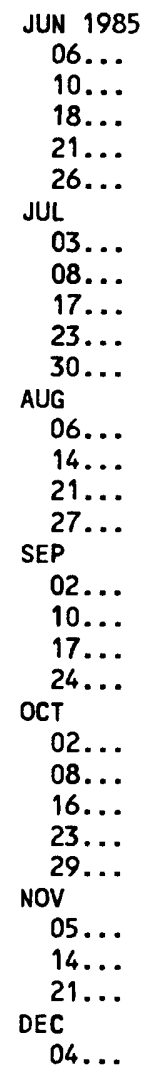

3.

$3.2 \quad 0.020$

$3.1 \quad 0.020$

-.

$3.8 \quad 0.030$

$2.3 \quad 0.020$

0.02

0.02

0.03

0.02

$--$

2.4

0.030

-. $\quad$-.

$\begin{array}{ll}- & - \\ - & -\end{array}$

$3.2 \quad 0.00$

$\begin{array}{ll}2 & 0.00 \\ -- & -. \\ -- & -.\end{array}$

$2.4 \quad 0.030$

$-\cdot$

-.

$\begin{array}{ll}-- & - \\ -- & -\end{array}$

0.03

$\begin{array}{lll}1 .-2 & - \\ -- & 0.040\end{array}$

1.90 .040

-. -.

$-$

$2.1 \quad 0.030$

$\begin{array}{ll}-. & -- \\ -. & -.\end{array}$

$2.3 \quad 0.030$

0.03

0.03

0.04

0.04

-.

02
--
--

$\cdots$

729

616
568
--
573
646

390

350

380

410

634

410

$$
\text { -. }
$$$$
\text { -. }
$$

789

$789 \quad 470$

470

$$
\text { -. }
$$$$
\text { -- }
$$

460

460

$$
-
$$$$
-
$$$$
675
$$

675

-.

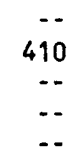

410

744

430
--
--

720

410

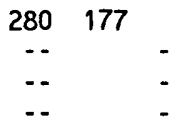

$\begin{array}{ccc}220 & 168 & \\ 200 & 145 & \\ -- & & \cdots \\ 220 & 159 & \\ 230 & 174 & \end{array}$

81
71
81
86

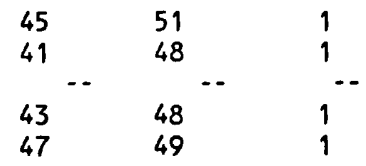

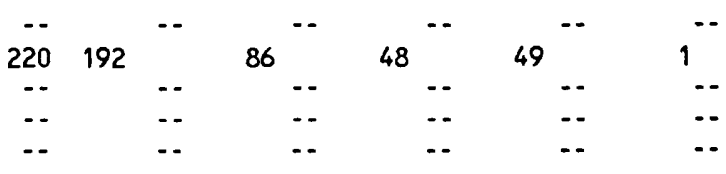

$300 \quad 171$

99

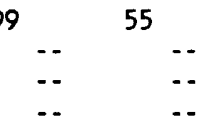

69

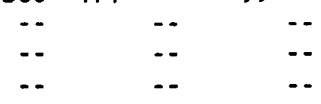

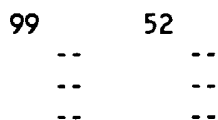

$230 \quad 178$

-. $178 \quad \ldots$

-.

$85^{--}$

$47^{-}$

$\begin{array}{cc}54 & \\ & 1 \\ \cdots & -- \\ -- & --\end{array}$

\section{$270 \quad 167$}

91

-.

50

67

89

46

70

2 
Table 34.--Chemical analyses of surface-water samples collected at Whitewood Creek above Whitewood, S. Dak.-- Cont inued

WATER QUALITY DATA

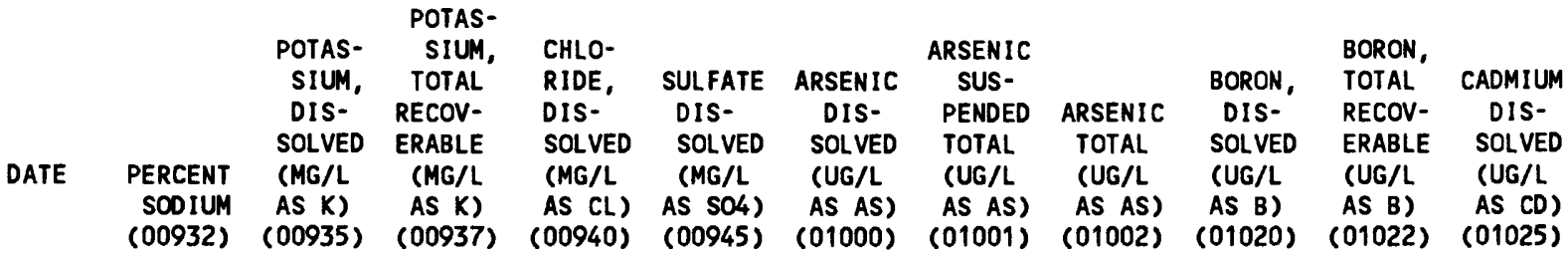

JUN 1985

$06 . .$.

$10 \ldots$

$18 . .$.

$21 \ldots$

26...

JUL

$03 . .$.

$08 .$.

$17 . .$.

23...

$30 .$.

AUG

06...

$14 \ldots$

21...

$27 .$.

SEP

$02 .$.

$10 .$.

$17 . .$.

$24 \ldots$

OCT

$02 . .$.

$08 .$.

$16 . .$.

$23 .$.

NOV

$05 . .$.

$14 \ldots$

DEC

04 ...

$\begin{array}{lllllll}22 & 12 & . & 13 & 18 & 260 & 3 \\ 22 & 11 & 10 & 13 & 250 & 30 \\ -- & & \ldots & -- & & -- & \\ 21 & 9.4 & 9.2 & 12 & 240 & 3 \\ 20 & 9.6 & 9.5 & 17 & 280 & 43\end{array}$

$\begin{array}{rrrrrr}31 & 23 & 54 & 100 & 100 & <1 \\ 30 & 32 & 62 & 90 & 90 & <1 \\ -- & -- & -- & -- & -- & -- \\ 33 & 40 & 73 & 90 & 110 & <1 \\ 43 & -- & -- & 100 & 130 & <1\end{array}$

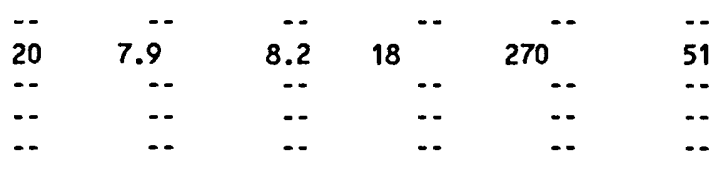

$\begin{array}{ccccccc}23 & 12 & & 12 & 16 & 380 & 75 \\ -- & \ldots & \ldots & \ldots & \ldots & - \\ \ldots & \ldots & -. & \ldots & \ldots & - \\ -. & \ldots & -. & \ldots & \ldots & -\end{array}$

.-
51
$\cdots$
$\cdots$
-
75
$\cdots$
-

$\begin{array}{crrrrrr}22 & 11 & & 12 & 17 & 340 & 46 \\ -. & \ldots & \ldots & \ldots & \ldots & \\ -. & & \ldots & \ldots & \ldots & \ldots & -\end{array}$

$46 \quad 4 \quad 50 \quad 120 \quad 170 \quad<1$

$\begin{array}{llllll}- & \ldots & \ldots & - & \ldots & \ldots \\ -. & \ldots & \ldots & \ldots & \ldots & \ldots\end{array}$

$\begin{array}{ccccccc}-. & -. & -. & -. & -. & - \\ 22 & 9.7 & 9.9 & 19 & 290 & 3 \\ -- & -. & -. & -. & -. & - \\ -. & -. & -. & -. & -. & - \\ -- & -. & -. & -- & -- & -\end{array}$

$\begin{array}{crrrrrr}24 & 13 & & 11 & 18 & 360 & 31 \\ .- & \ldots & \ldots & \ldots & \ldots & - \\ .- & \ldots & \ldots & \ldots & \ldots & .\end{array}$

-
35
--
--
31
--

$\begin{array}{lllll}26 & 14 & 16 & 25 & 350\end{array}$

22

--
4
$\cdots$
-
1
-

3

$\begin{array}{lll}110 & 110 & -.\end{array}$

$10 \quad 110 \quad<1$

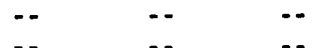

$\begin{array}{lll}- & - & - \\ -- & - & -\end{array}$

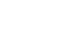

16

350

34

120

$\begin{array}{ccc}120 & 140 & <1 \\ -- & \cdots & \cdots \\ -- & \cdots & \cdots\end{array}$ 
Table 34.-- Chemical analyses of surface-water samples collected at Whitewood Creek above Whitewood, S. Dak.--Cont inued

\section{WATER QUALITY DATA}

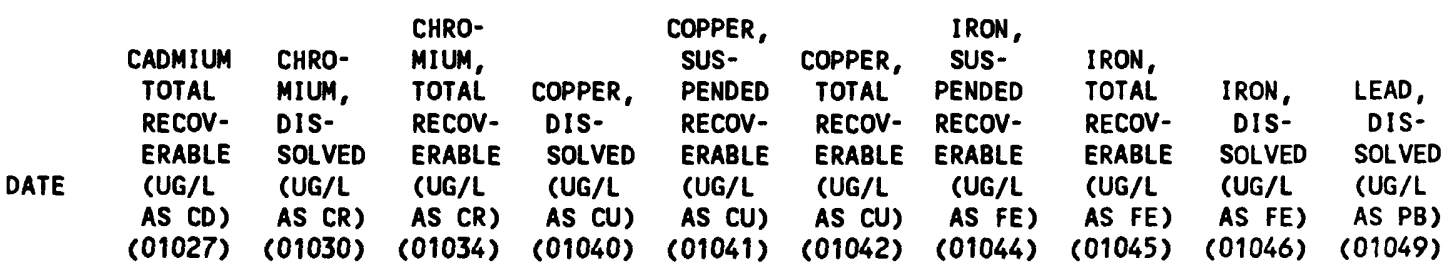

\section{JUN 1985}

$06 \ldots$

$10 . .$.

$18 . .$.

$21 \ldots$

26...

JUL

$03 . .$.

$08 . .$.

$17 .$.

23...

$30 . .$.

AUG

$06 . .$.

$14 . .$.

$21 \ldots$

$27 .$.

02...

$10 . .$.

$17 . .$.

$24 \ldots$

OCT

$02 . .$.

$08 . .$.

$16 \ldots$

23...

$29 . .$.

NOV

$05 .$. .

$14 \ldots$

$21 \ldots$ DEC

$04 \ldots$

$\begin{array}{rrr}<1 & <10 & <10 \\ <1 & <10 & <10 \\ -- & -- & - \\ <1 & <10 & 10 \\ 1 & <10 & <10\end{array}$

7
7
-5
3

10
13
--
27
25

17
20
$\cdots$
32
28

1900
3900
--
5900
5200

1900

3900

$$
5900
$$

5200

$\begin{array}{rr}6 & <1 \\ 9 & <1 \\ -- & -- \\ 17 & 5 \\ 8 & 5\end{array}$

$<1 \quad<10$

$\begin{array}{rrr}-- & -- & - \\ <10 & 5 & 3\end{array}$

$-$

$\cdots$

$\begin{array}{ll}<1 & <10 \\ \therefore- & -- \\ -- & -- \\ -- & --\end{array}$

$<10$
--

$<1<10<10$

$\begin{array}{ccc}< & <10 & <10 \\ -- & -- & --\end{array}$

$\begin{array}{ll}-- & - \\ -- & --\end{array}$

-.

$<1 \quad<10$

$-$

--

$<1<10$

$\begin{array}{ll}<1 & <10 \\ -- & -- \\ -- & --\end{array}$

$<10$

$1<10$

$<10$

3 $\begin{array}{rrr}-- & -- & -. \\ 8 & 230 & 230 \\ -- & -- & --\end{array}$

$\begin{aligned}-. & - \\ 5 & <1 \\ -- & - \\ -- & - \\ -- & -. \\ & \end{aligned}$

$6<1$

$130 \quad 140$

$\begin{array}{ll}-- & -- \\ -- & --\end{array}$

--

$-$

$440 \quad 450$

$\begin{array}{ll}440 & -- \\ -- & -.\end{array}$

$11<1$

-- $\quad-\cdot$

-.

$\begin{array}{cccc}-- & -- & -. & - \\ 730 & 740 & 13 & 1 \\ -. & -- & -. & -.\end{array}$

-.

$\cdots$

$-\cdot$

7

$7 \quad 1$

$\begin{array}{ll}2 & 3 \\ - & \cdots \\ 1 & -\end{array}$

$150 \quad 160$

$\begin{array}{ll}150 & -. \\ -. & -.\end{array}$

290
10

$<1$ 
Table 34.--Chemical analyses of surface-water samples collected at whitewood Creek above Whitewood, S. Dak.--Cont inued

WATER QUALITY DATA

\begin{tabular}{|c|c|c|c|c|c|c|c|c|c|c|}
\hline ATE & $\begin{array}{l}\text { LEAD, } \\
\text { SUS- } \\
\text { PENDED } \\
\text { RECOV- } \\
\text { ERABLE } \\
\text { (UG/L } \\
\text { AS PB) } \\
(01050)\end{array}$ & $\begin{array}{l}\text { LEAD, } \\
\text { TOTAL } \\
\text { RECCV- } \\
\text { ERABLE } \\
\text { (UG/L } \\
\text { AS PB) } \\
(01051)\end{array}$ & $\begin{array}{l}\text { MANGA- } \\
\text { NESE, } \\
\text { SUS- } \\
\text { PENDED } \\
\text { RECOV. } \\
\text { (UG/L } \\
\text { AS MN) } \\
(01054)\end{array}$ & $\begin{array}{l}\text { MANGA- } \\
\text { NESE, } \\
\text { TOTAL } \\
\text { RECOV- } \\
\text { ERABLE } \\
\text { (UG/L } \\
\text { AS MN) } \\
(01055)\end{array}$ & $\begin{array}{l}\text { MANGA- } \\
\text { NESE, } \\
\text { DIS- } \\
\text { SOLVED } \\
\text { (UG/L } \\
\text { AS MN) } \\
(01056)\end{array}$ & $\begin{array}{c}\text { MERCURY } \\
\text { DIS- } \\
\text { SOLVED } \\
\text { (UG/L } \\
\text { AS HG) } \\
(71890)\end{array}$ & $\begin{array}{l}\text { MERCURY } \\
\text { TOTAL } \\
\text { RECCV- } \\
\text { ERABLE } \\
\text { (UG/L } \\
\text { AS HG) } \\
(71900)\end{array}$ & $\begin{array}{c}\text { SI LVER, } \\
\text { DIS- } \\
\text { SOLVED } \\
\text { (UG/L } \\
\text { AS AG) } \\
(01075)\end{array}$ & $\begin{array}{l}\text { SILVER, } \\
\text { TOTAL } \\
\text { RECOV- } \\
\text { ERABLE } \\
\text { (UG/L } \\
\text { AS AG) } \\
(01077)\end{array}$ & $\begin{array}{l}\text { ZINC, } \\
\text { DIS- } \\
\text { SOLVED } \\
\text { (UG/L } \\
\text { AS ZN) } \\
(01090)\end{array}$ \\
\hline
\end{tabular}

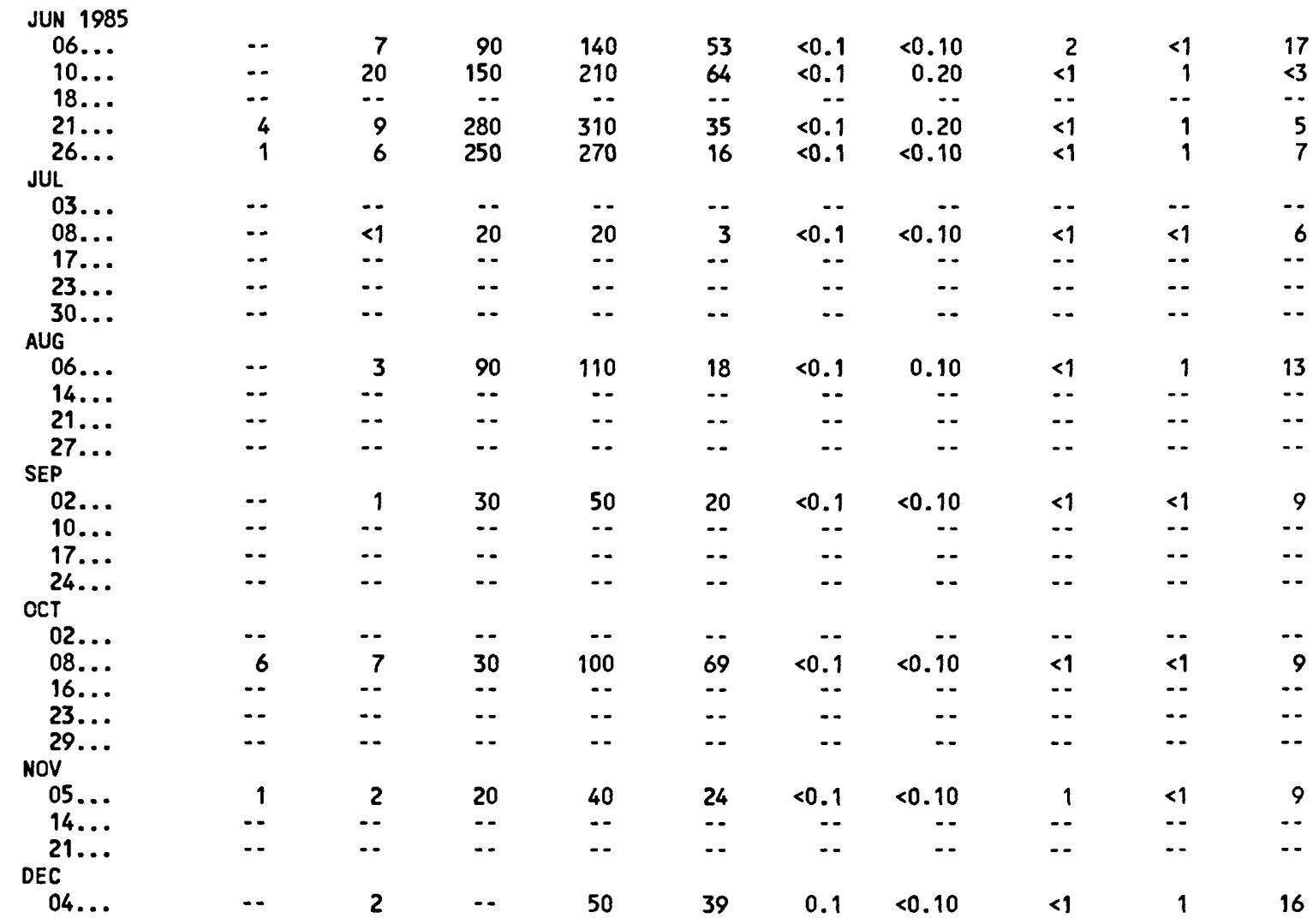


Table 34.--Chemical analyses of surface-water samples collected at Whitewood Creek above Whitewood, S. Dak.--Cont inued

WATER QUALITY DATA

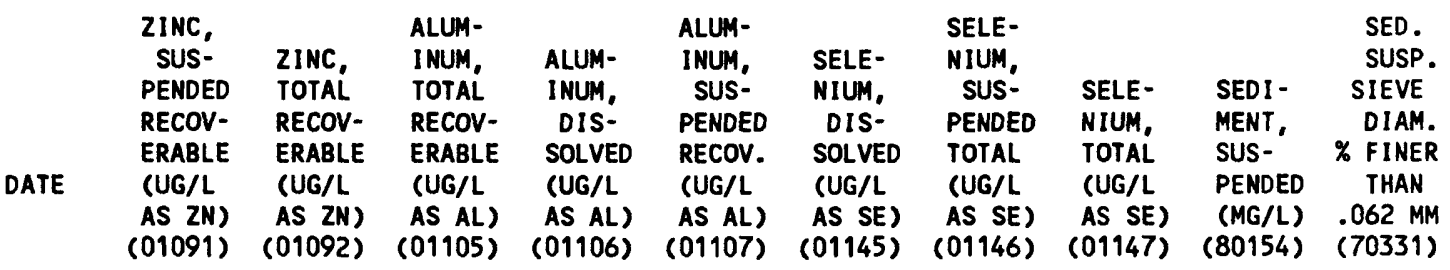

JUN 1985

$06 . .$.

$10 .$.

$18 . .$.

$21 . .$.

26... JUL

$03 . .$.

$08 . .$.

$17 \ldots$

$23 \ldots$

AUG

$06 . .$.

$14 \ldots$

$21 . .$.

$27 . .$.

SEP

$02 . .$.

$10 . .$.

$17 . .$.

$24 \ldots$

OCT

$02 . .$.

$08 . .$.

$16 \ldots$

23...

$29 . .$.

NOV

$05 .$. .

$14 \ldots$ DEC

$04 .$.

$\begin{array}{rrrrr}30 & 50 & 360 & 40 & 320 \\ -- & 50 & 680 & 40 & 640 \\ -- & -. & -. & -- & -. \\ 60 & 60 & 1100 & 30 & 1100 \\ 50 & 60 & 970 & 20 & 950\end{array}$

2
2
--
2
2

\begin{tabular}{cc}
0 & \\
0 & \\
\hdashline- & - \\
-- & - \\
-- & -
\end{tabular}

$\begin{array}{rrr}1 & 72 & 100 \\ 1 & 81 & 98 \\ \therefore- & 51 & 99 \\ \therefore- & 73 & 94 \\ -- & 106 & 96\end{array}$

$10 \quad 20$

$\overline{90} \quad 30$

60

$\begin{array}{ccc}-- & -- & - \\ 2 & -- & -\end{array}$

$\begin{array}{llll}-- & -- & -\end{array}$

$--$

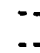

2

$\begin{array}{llll}0 & 2 & 17 & 92\end{array}$

$7 \quad 20 \quad<10$

40

$+$

-.

$-$

$\begin{array}{lll}-- & - & - \\ -- & -- & --\end{array}$

- $\quad-$.

$10 \quad 20 \quad 100$

$80 \quad 20$

$\begin{array}{lll}10 & -. & -- \\ \cdots & - & -\end{array}$

$\begin{array}{lll}80 & - \\ -- & --\end{array}$

- $\quad \ldots-1$

-.

--

10

240

$\begin{array}{lll}-- & -\cdot\end{array}$

- $\quad \ldots \quad$-.

$1 \quad 10 \quad 50$

50
--
--

-.

30

210

-.

-.

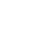

$20 \quad 30$

--

40

$<10 \quad-$.

20

3

$\cdots$

3

3 
Table 34.--Chemical analyses of surface-water samples collected at Whitewood Creek above Whitewood, S. Dak.--Cont inued

\section{WATER QUALITY DATA}

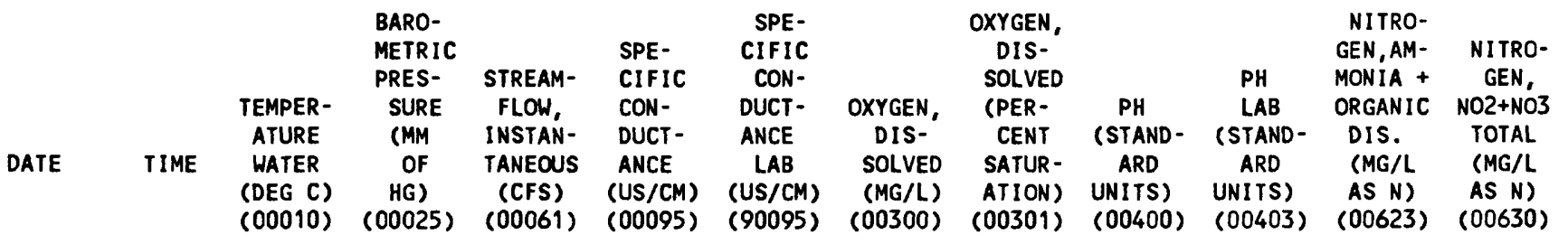

\begin{tabular}{|c|c|c|c|c|c|c|c|c|c|c|c|c|}
\hline \multicolumn{13}{|l|}{ FEB 1986} \\
\hline $06 \ldots$ & 1000 & 0.5 & 667 & 19 & 953 & 986 & 12.3 & 98 & -- & 8.20 & -- & -- \\
\hline $21 \ldots$ & 1015 & -- & -. & 16 & -- & 990 & - & -- & -. & 8.10 & -. & - \\
\hline $25 \ldots$ & 1115 & 4.5 & 668 & 25 & 767 & 848 & 10.2 & 90 & 8.30 & 8.20 & 0.90 & - \\
\hline $26 \ldots$ & 1530 & -- & -. & 28 & -- & -. & -. & -- & -- & -- & -- & - \\
\hline $27 \ldots$ & 1440 & -- & -- & 25 & -- & $\cdots$ & -. & -- & - & $-\cdot$ & $\cdots$ & $\ldots$ \\
\hline $28 \ldots$ & 1345 & -- & $\cdots$ & 21 & -- & $\cdots$ & $\cdots$ & -- & $\cdots$ & -. & -- & $\cdots$ \\
\hline \multicolumn{13}{|l|}{ MAR } \\
\hline $03 \ldots$ & 1015 & $\cdots$ & -- & 24 & -- & $\cdots$ & $\cdots$ & $\cdots$ & -- & -- & $\cdots$ & $\cdots$ \\
\hline $05 \ldots$ & 1415 & $\cdots$ & -- & 26 & $\cdots$ & $\cdots$ & $\cdots$ & -. & -- & $\cdots$ & $-\cdot$ &.- \\
\hline $10 \ldots$ & 1030 & $\cdots$ & -- & 26 & -- & -- & $\cdots$ & -- & -- & $\cdots$ & $\cdots$ & - \\
\hline \multicolumn{13}{|l|}{ APR } \\
\hline $06 \ldots$ & 1318 & 11.0 & 667 & 47 & 582 & 595 & 8.4 & 87 & 8.60 & 8.20 & 0.40 & $\cdots$ \\
\hline $23 \ldots$ & 1330 & 10.0 & 663 & 165 & 305 & -. & 9.0 & 92 & 8.20 & -- & -. & \\
\hline $28 \ldots$ & 1215 & -- & -- & 115 & -. & -- & -. & -- & -. & -- & $\cdots$ & -6 \\
\hline \multicolumn{13}{|l|}{ MAY } \\
\hline $20 \ldots$ & 1055 & 13.0 & 670 & 42 & 467 & $\cdots$ & 11.5 & 125 & 9.20 & $\cdots$ & $\cdots$ & $\cdots$ \\
\hline \multicolumn{13}{|l|}{ JUN } \\
\hline $04 \ldots$ & 1315 & 17.5 & 671 & 49 & 548 & -- & 8.3 & 99 & 8.90 & $\cdots$ & $\cdots$ & 2.10 \\
\hline $09 \ldots$ & 1435 & 15.0 & 670 & 39 & 572 & -- & 8.5 & 96 & 8.50 & -- & -- & \\
\hline $09 \ldots$ & 1855 & -- & $\cdots$ & 96 & -- & $-\cdot$ & -- & -- & $=$ & -- & -. & \\
\hline $09 \ldots$ & 1955 & 14.0 & 668 & 102 & 484 & 496 & 8.8 & 98 & 8.40 & 7.90 & 0.50 & 2.10 \\
\hline $10 \ldots$ & 0030 & 13.5 & 669 & 96 & 426 & -. & 8.6 & 94 & 8.20 & -- & -. & 1.40 \\
\hline $10 \ldots$ & 1515 & -. & $\cdots$ & 178 & - & -. & - & $\ldots$ & -. & - & $\cdots$ & 0.800 \\
\hline \multicolumn{13}{|l|}{ JUL } \\
\hline $17 \ldots$ & 1330 & 25.0 & 668 & 16 & 829 & 780 & 8.2 & 114 & 8.80 & 8.80 & 0.70 & - \\
\hline \multirow{2}{*}{\multicolumn{13}{|c|}{$\begin{array}{l}\text { AUG } \\
12 \ldots\end{array}$}} \\
\hline & 1250 & 21.5 & 667 & 19 & 861 & 853 & 8.5 & 111 & 9.10 & 9.00 & 0.50 & $\cdots$ \\
\hline \multicolumn{13}{|l|}{ SEP } \\
\hline $18 . .$. & 1300 & 14.0 & 668 & 14 & 926 & 897 & 9.6 & 107 & 8.80 & 8.60 & 0.70 & \\
\hline
\end{tabular}


Table 34.--Chemical analyses of surface-water samples collected at whitewood Creek above Whitewood, S. Dak.--Cont inued

WATER QUALITY DATA

\begin{tabular}{|c|c|c|c|c|c|c|c|c|c|c|c|c|}
\hline ATE & $\begin{array}{l}\text { NITRO- } \\
\text { GEN, } \\
\text { NO2+NO3 } \\
\text { DIS- } \\
\text { SOLVED } \\
\text { (MG/L } \\
\text { AS N) } \\
\text { (00631) }\end{array}$ & $\begin{array}{l}\text { PHOS- } \\
\text { PHORUS, } \\
\text { TOTAL } \\
\text { (MG/L } \\
\text { AS P) } \\
(00665)\end{array}$ & $\begin{array}{l}\text { PHOS- } \\
\text { PHORUS, } \\
\text { DIS- } \\
\text { SOLVED } \\
\text { (MG/L } \\
\text { AS P) } \\
(00666)\end{array}$ & $\begin{array}{l}\text { CARBON, } \\
\text { ORGANIC } \\
\text { TOTAL } \\
\text { (MG/L } \\
\text { AS C) } \\
(00680)\end{array}$ & $\begin{array}{l}\text { CARBON, } \\
\text { ORGANIC } \\
\text { DIS- } \\
\text { SOLVED } \\
\text { (MG/L } \\
\text { AS C) } \\
(00681)\end{array}$ & $\begin{array}{c}\text { CYANIDE } \\
\text { TOTAL } \\
\text { (MG/L } \\
\text { AS CN) } \\
(00720)\end{array}$ & $\begin{array}{l}\text { CYANIDE } \\
\text { DIS- } \\
\text { SOLVED } \\
\text { (MG/L } \\
\text { AS CN) } \\
(00723)\end{array}$ & $\begin{array}{l}\text { SOLIDS, } \\
\text { RESIDUE } \\
\text { AT } 180 \\
\text { DEG. C } \\
\text { DIS- } \\
\text { SOLVED } \\
(M G / L) \\
(70300)\end{array}$ & $\begin{array}{l}\text { SOLIDS, } \\
\text { SUM OF } \\
\text { CONST I- } \\
\text { TUENTS, } \\
\text { DIS- } \\
\text { SOLVED } \\
(M G / L) \\
(70301)\end{array}$ & $\begin{array}{l}\text { HARD- } \\
\text { NESS } \\
\text { (MG/L } \\
\text { AS } \\
\text { CACO3) } \\
(00900)\end{array}$ & $\begin{array}{l}\text { HARD- } \\
\text { NESS } \\
\text { NONCAR- } \\
\text { BONATE } \\
\text { (MG/L } \\
\text { AS } \\
\text { CACO3) } \\
(95902)\end{array}$ & $\begin{array}{c}\text { ALKA- } \\
\text { LINITY } \\
\text { LAB } \\
\text { (MG/L } \\
\text { AS } \\
\text { CACO3) } \\
(90410)\end{array}$ \\
\hline
\end{tabular}

\begin{tabular}{|c|c|c|c|c|c|c|c|c|c|c|c|c|c|}
\hline FEB 1986 & & & & & & & & & & & & & \\
\hline $06 \ldots$ & $\cdots$ & $\cdots$ & $\cdots$ & 1.9 & 1.9 & 0.130 & 0.14 & 673 & $\cdots$ & 360 & 210 & 143 & \\
\hline $21 \ldots$ & -. & -. & - & .. & -. & 0.190 & 0.19 & 671 & $\cdots$ & 380 & 230 & 153 & \\
\hline $25 \ldots$ & 6.00 & $\cdots$ & 0.160 & $\cdots$ & -. & - & 0.14 & 559 & -- & - & $\cdots$ & 136 & \\
\hline $26 .$. & -- & $\cdots$ & -- & - & $\cdots$ & -. & -- & -. & $\cdots$ & $\ldots$ & $\cdots$ & & $\cdots$ \\
\hline $27 \ldots$ & -. & $\cdots$ & $\cdots$ & $\cdots$ & $\cdots$ & -. & -. & -- & $\cdots$ & -- & $\cdots$ & & $\cdots$ \\
\hline 28... & .. & $\cdots$ & -- & $\cdots$ & - & -- & -- & - & $\cdots$ & $\cdots$ & $\cdots$ & & $\cdots$ \\
\hline MAR & & & & & & & & & & & & & \\
\hline $03 \ldots$ & $\cdots$ & $\cdots$ & $\cdots$ & $\cdots$ & $\cdots$ & $\cdots$ & $\cdots$ & $\cdots$ & $\cdots$ & -- & $\cdots$ & & $\cdots$ \\
\hline $05 \ldots$ & -- & -- & - & $\cdots$ & $\cdots$ & $\cdots$ & $\cdots$ & $\cdots$ & $\cdots$ & -- & $\cdots$ & & $\cdots$ \\
\hline${ }_{A P R}^{10 \ldots .}$ & $\cdots$ & - & $\cdots$ & $\cdots$ & -- & $\cdots$ & -- & $\cdots$ & $\cdots$ & $\cdots$ & $\cdots$ & & $\cdots$ \\
\hline $06 \ldots$ & 3.10 & -. & $\cdots$ & 5.6 & 2.7 & -- & 0.06 & 377 & 340 & 240 & 130 & 115 & \\
\hline $23 \ldots$ & - & $\cdots$ & $\cdots$ & -. & -. & $\cdots$ & -. & -. & -. & -. & -- & & $\cdots$ \\
\hline 28... & $\cdots$ & $\cdots$ & $\cdots$ & $\cdots$ & $\cdots$ & $\cdots$ & $\cdots$ & -- & $\cdots$ & $\cdots$ & $\cdots$ & & $\cdots$ \\
\hline MAY & & & & & & & & & & & & & \\
\hline $20 \ldots$ & $\cdots$ & $\cdots$ & $\cdots$ & $-\cdot$ & $\cdots$ & -- & -- & $\cdots$ & $\cdots$ & $\cdots$ & $\cdots$ & & $\cdots$ \\
\hline JUN & & & & & & & & & & & & & \\
\hline $04 \ldots$ & $-\cdot$ & 0.610 & $\cdots$ & 7.6 & $\cdots$ & -- & $\cdots$ & $\cdots$ & -- & $\cdots$ & $\cdots$ & & -. \\
\hline $09 \ldots$ & $\cdots$ & - & $\cdots$ & -. & $\cdots$ & $\cdots$ & $\cdots$ & $\cdots$ & $\cdots$ & $\cdots$ & $\cdots$ & & -- \\
\hline $09 .$. & .. & -- & $\cdots$ & $\cdots$ & -- & $\cdots$ & -- & -. & -. & -. & $\cdots$ & & $\cdots$ \\
\hline $09 .$. & 2.00 & 0.610 & 0.140 & 39 & - & $\cdots$ & 0.02 & 313 & 310 & 220 & 91 & 129 & \\
\hline $10 \ldots$ & - & 1.20 & -- & 18 & $\cdots$ & $\cdots$ & -. & -- & -. & -. & $\cdots$ & & $\cdots$ \\
\hline $10 \ldots$ & -. & 0.610 & $\cdots$ & 14 & - & -. & -. & -. & - & -- & $\cdots$ & & -- \\
\hline JUL & & & & & & & & & & & & & \\
\hline $17 \ldots$ & 5.60 & $\cdots$ & 0.196 & 2.7 & 2.1 & $\cdots$ & 0.01 & 568 & 540 & 350 & 200 & 157 & \\
\hline AUG & & & & & & & & & & & & & \\
\hline $12 \ldots$ & 7.00 & $\cdots$ & 0.300 & 2.3 & 2.8 & -- & 0.02 & 621 & 590 & 380 & 230 & 151 & \\
\hline $18 \ldots$ & 7.60 & $\cdots$ & 0.220 & 2.1 & 2.0 & $\cdots$ & 0.03 & 730 & 620 & 400 & 250 & 148 & \\
\hline
\end{tabular}


Table 34.--Chemical analyses of surface-water samples collected at whitewood Creek above Whitewood, S. Dak.--Cont inued

WATER QUALITY DATA

\begin{tabular}{|c|c|c|c|c|c|c|c|c|c|c|c|c|}
\hline DATE & $\begin{array}{l}\text { CALCIUM } \\
\text { DIS- } \\
\text { SOLVED } \\
\text { (MG/L } \\
\text { AS CA) } \\
(00915)\end{array}$ & $\begin{array}{l}\text { MAGNE- } \\
\text { SIUM, } \\
\text { DIS-- } \\
\text { SOLVED } \\
\text { (MG/L } \\
\text { AS MG) } \\
(00925)\end{array}$ & $\begin{array}{l}\text { SOOIUM, } \\
\text { DIS- } \\
\text { SOLVED } \\
\text { (MG/L } \\
\text { AS NA) } \\
(00930)\end{array}$ & $\begin{array}{c}\text { SOOIUM } \\
\text { AD- } \\
\text { SORP- } \\
\text { TION } \\
\text { RATIO } \\
(00931)\end{array}$ & $\begin{array}{l}\text { PERCENT } \\
\text { SOD IUM } \\
(00932)\end{array}$ & $\begin{array}{l}\text { POTAS- } \\
\text { SIUM, } \\
\text { DIS- } \\
\text { SOLVED } \\
\text { (MG/L } \\
\text { AS K) } \\
(00935)\end{array}$ & $\begin{array}{l}\text { POTAS- } \\
\text { SIUM, } \\
\text { TOTAL } \\
\text { RECOV- } \\
\text { ERABLE } \\
\text { (MG/L } \\
\text { AS K) } \\
(00937)\end{array}$ & $\begin{array}{l}\text { CHLO- } \\
\text { RIDE, } \\
\text { DIS- } \\
\text { SOLVED } \\
\text { (MG/L } \\
\text { AS CL) } \\
(00940)\end{array}$ & $\begin{array}{l}\text { SULFATE } \\
\text { DIS- } \\
\text { SOLVED } \\
\text { (MG/L } \\
\text { AS SO4) } \\
(00945)\end{array}$ & $\begin{array}{l}\text { FLUO- } \\
\text { RIDE, } \\
\text { DIS- } \\
\text { SOLVED } \\
\text { (MG/L } \\
\text { AS F) } \\
(00950)\end{array}$ & $\begin{array}{l}\text { SILICA, } \\
\text { DIS- } \\
\text { SOLVED } \\
\text { (MG/L } \\
\text { AS } \\
\text { SIO2) } \\
(00955)\end{array}$ & $\begin{array}{c}\text { ARSENIC } \\
\text { DIS- } \\
\text { SOLVED } \\
\text { (UG/L } \\
\text { AS AS) } \\
(01000)\end{array}$ \\
\hline
\end{tabular}

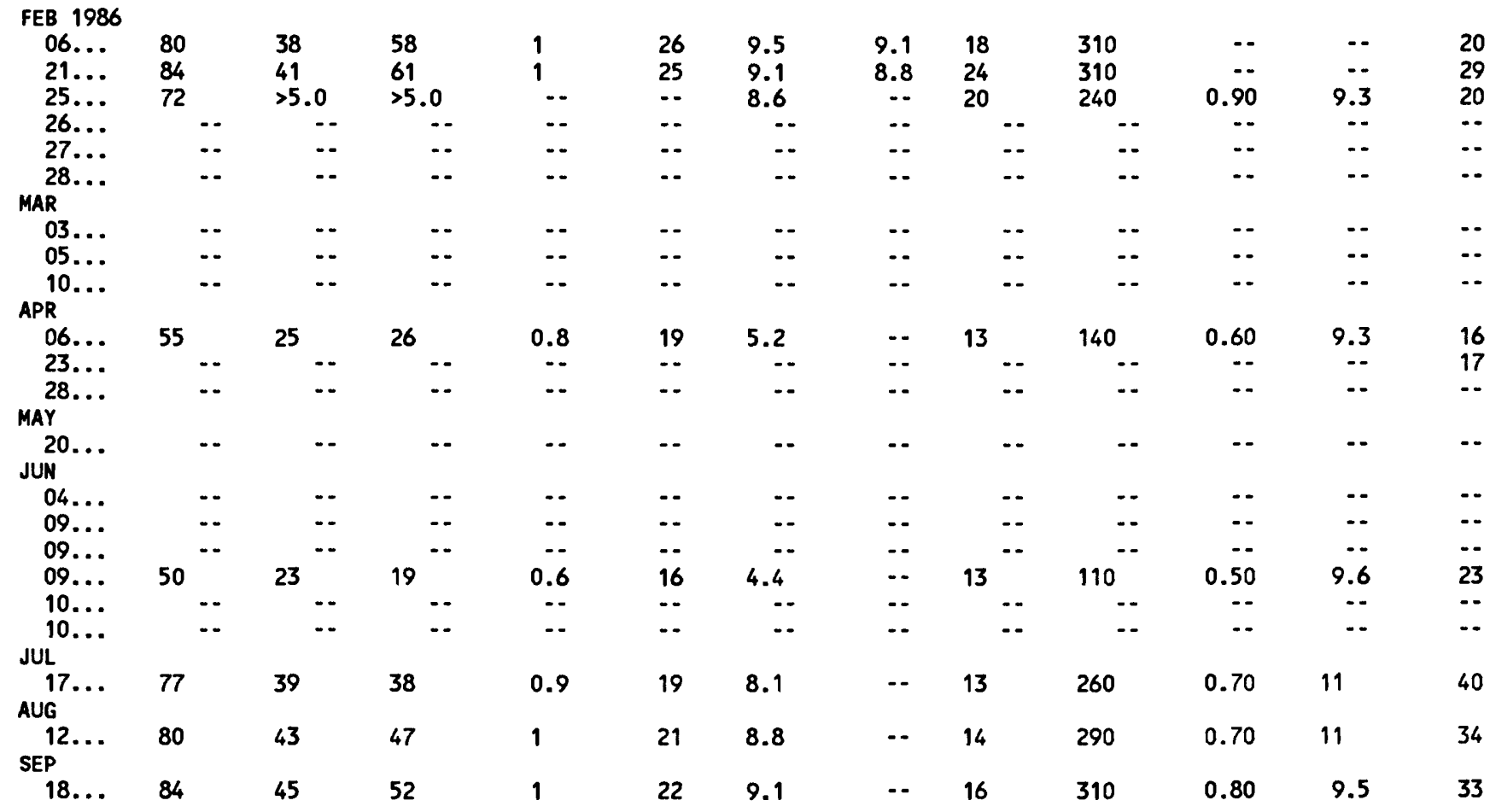


Table 34.--Chemical analyses of surface-water samples collected at Whitewood Creek above Whitewood, S. Dak.--Cont inued

WATER QUALITY DATA

\begin{tabular}{|c|c|c|c|c|c|c|c|c|c|c|c|c|}
\hline DATE & $\begin{array}{c}\text { ARSENIC } \\
\text { TOTAL } \\
\text { (UG/L } \\
\text { AS AS) } \\
(01002)\end{array}$ & $\begin{array}{l}\text { BORON, } \\
\text { DIS- } \\
\text { SOLVED } \\
\text { (UG/L } \\
\text { AS B) } \\
(01020)\end{array}$ & $\begin{array}{l}\text { BORON, } \\
\text { TOTAL } \\
\text { RECOV- } \\
\text { ERABLE } \\
\text { (UG/L } \\
\text { AS B) } \\
(01022)\end{array}$ & $\begin{array}{c}\text { CADMIUM } \\
\text { DIS- } \\
\text { SOLVED } \\
\text { (UG/L } \\
\text { AS CD) } \\
(01025)\end{array}$ & $\begin{array}{l}\text { CADMIUM } \\
\text { TOTAL } \\
\text { RECOV- } \\
\text { ERABLE } \\
\text { (UG/L } \\
\text { AS CD) } \\
(01027)\end{array}$ & $\begin{array}{l}\text { CHRO- } \\
\text { MIUM, } \\
\text { DIS- } \\
\text { SOLVED } \\
\text { (UG/L } \\
\text { AS CR) } \\
(01030)\end{array}$ & $\begin{array}{l}\text { CHRO- } \\
\text { MIUM, } \\
\text { TOTAL } \\
\text { RECOV- } \\
\text { ERABLE } \\
\text { (UG/L } \\
\text { AS CR) } \\
(01034)\end{array}$ & $\begin{array}{l}\text { COPPER, } \\
\text { DIS- } \\
\text { SOLVED } \\
\text { (UG/L } \\
\text { AS CU) } \\
(01040)\end{array}$ & $\begin{array}{c}\text { COPPER, } \\
\text { TOTAL } \\
\text { RECOV- } \\
\text { ERABLE } \\
\text { (UG/L } \\
\text { AS CU) } \\
(01042)\end{array}$ & $\begin{array}{l}\text { IRCN, } \\
\text { TOTAL } \\
\text { RECOV- } \\
\text { ERABLE } \\
\text { (UG/L } \\
\text { AS FE) } \\
(01045)\end{array}$ & $\begin{array}{l}\text { IRON, } \\
\text { DIS- } \\
\text { SOLVED } \\
\text { (UG/L } \\
\text { AS FE) } \\
(01046)\end{array}$ & $\begin{array}{l}\text { LEAD, } \\
\text { DIS- } \\
\text { SOLVED } \\
\text { (UG/L } \\
\text { AS PB) } \\
(01049)\end{array}$ \\
\hline
\end{tabular}

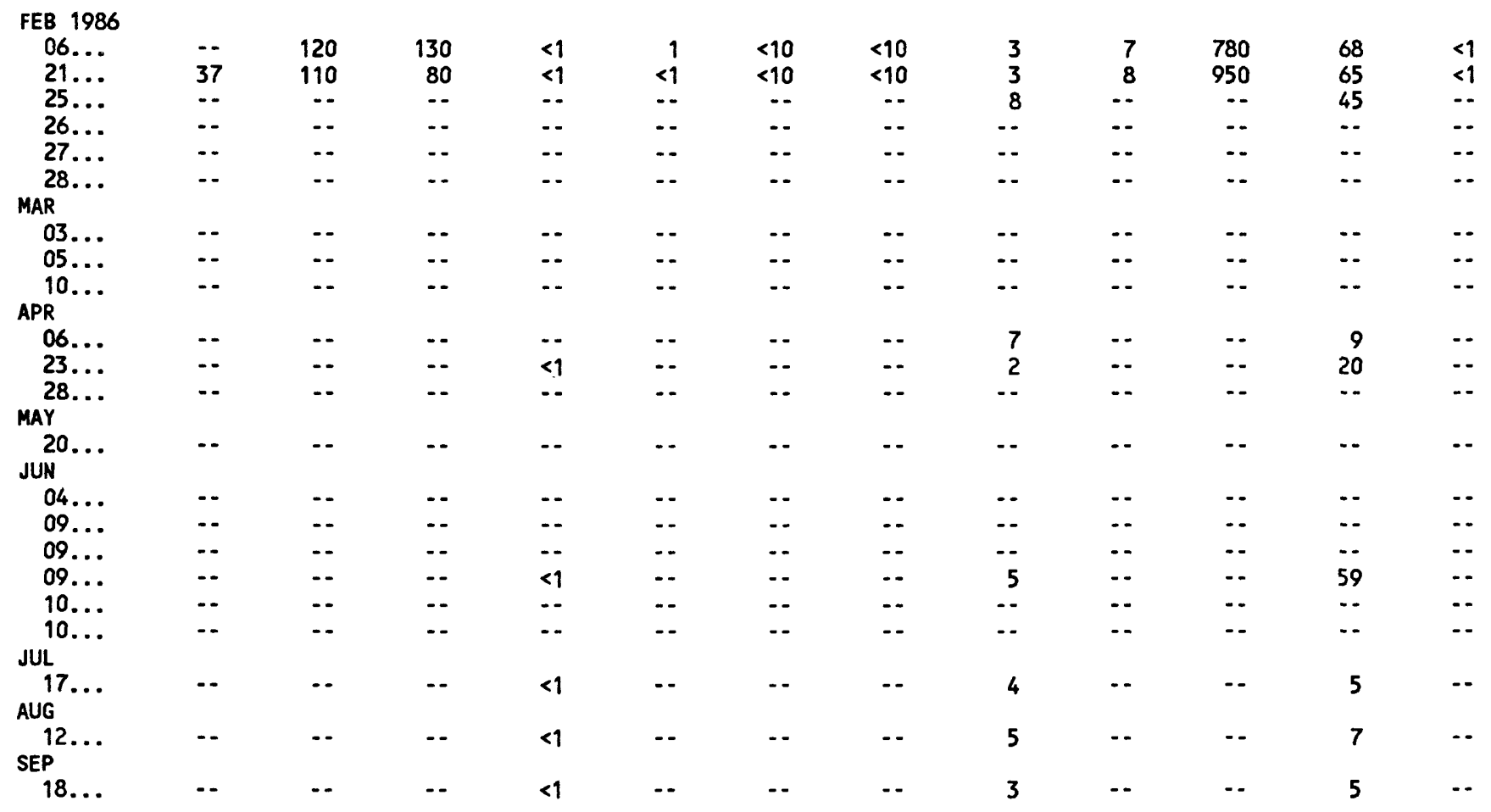


Table 34.-- Chemical analyses of surface-water samples collected at whitewood Creek above Whitewood, S. Dak. --Cont inued

\section{WATER QUALITY DATA}

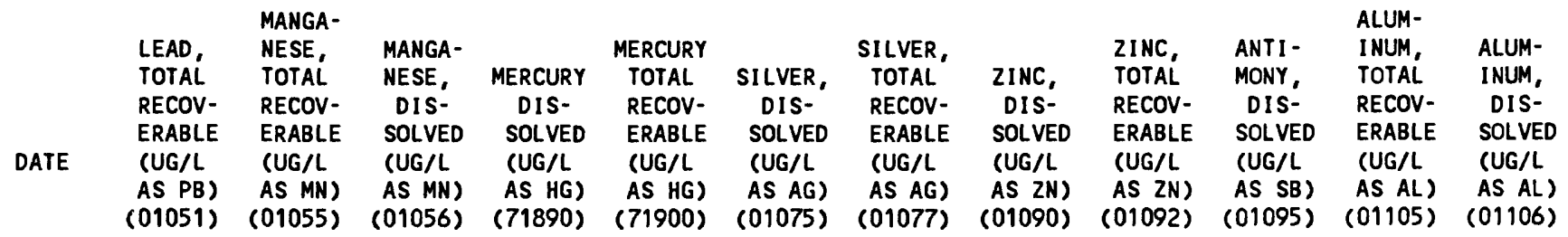

\begin{tabular}{|c|c|c|c|c|c|c|c|c|c|c|c|c|}
\hline FEB 1986 & & & & & & & & & & & & \\
\hline $06 \ldots$ & 1 & 170 & 120 & 0.1 & 0.20 & $<1$ & $<1$ & 6 & 70 & -- & 90 & 20 \\
\hline $21 \ldots$ & 1 & 210 & 150 & 0.1 & 0.30 & $<1$ & $<1$ & 4 & 30 & -- & 120 & 20 \\
\hline $25 \ldots$ & -- & -- & 120 & 0.1 & -- & $\cdots$ & $\cdots$ & $<3$ & $\cdots$ & 1 & -- & -- \\
\hline $26 \ldots$ & -- & -- & -- & -- & .. & $\ldots$ & -- & -- & -- & -- & -. & -- \\
\hline $27 \ldots$ & -- & -- & -- & -- & -- & - & -- & $\cdots$ & $\cdots$ & -. & -- & - \\
\hline $28 \ldots$ & -- & -- & -- & $\cdots$ & -- & $\cdots$ & -- & -- & -- & -- & -- & $\cdots$ \\
\hline MAR & & & & & & & & & & & & \\
\hline $03 \ldots$ & -- & -- & -- & -- & -- & -- & -- & -- & -- & $\cdots$ & -- & $\cdots$ \\
\hline $05 \ldots$ & -- & -. & $\cdots$ & $-\cdot$ & $\cdots$ & $\cdots$ & -- & -- & -- & $\cdots$ & $\cdots$ & - \\
\hline $10 \ldots$ & -- & $\cdots$ & -. & - & $-\cdot$ & -- & -- & -. & -- & - & -- & $\cdots$ \\
\hline APR & & & & & & & & & & & & \\
\hline $06 \ldots$ & -- & -- & 74 & $<0.1$ & 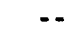 & -- & -- & $<3$ & $\cdots$ & 1 & $\cdots$ & -- \\
\hline $23 \ldots$ & -- & -- & 70 & $<0.1$ & -- & -- & - & $<10$ & -- & 1 & -- & $\cdots$ \\
\hline 28... & -- & $\cdots$ & -- & -. & $-\cdot$ & $\cdots$ & $\cdots$ & -- & -- & -- & $\cdots$ & -- \\
\hline MAY & & & & & & & & & & & & \\
\hline $20 \ldots$ & -- & -- & -- & -- & -- & -- & -- & -- & -- & -- & $\cdots$ & -- \\
\hline $\begin{array}{l}\text { JUN } \\
04 \ldots\end{array}$ & -- & -. & -. & -- & $\ldots$ & $\ldots$ & .- & -- & -- & .. & -- & - \\
\hline $09 .$. & $\cdots$ & - & - & - & -- & -- & -- & -. & -. & .- & -. & .. \\
\hline $09 . .$. & -. & .. & .. & - - & .. & .- & .- & .. & .- & -. & .- & -. \\
\hline $09 . .$. & -- & -- & 130 & $<0.1$ & -. & -. & -- & 5 & $\ldots$ & 2 & $\ldots$ & -. \\
\hline $10 \ldots$ & -. & -- & - & -. & -- & -- & -- & -. & $\ldots$ & - & -- & -- \\
\hline $10 \ldots$ & $-\cdot$ & - & -- & -. & -. & - - & -. & -- & -- & -. & - & -- \\
\hline JUL & & & & & & & & & & & & \\
\hline $17 \ldots$ & -- & -- & 11 & $<0.1$ & $\cdots$ & -- & -- & $<3$ & -- & 2 & -- & $\cdots$ \\
\hline AUG & & & & & & & & & & & & \\
\hline $12 \ldots$ & -- & $\cdots$ & 6 & $<0.1$ & $-\cdot$ & -- & -- & 7 & -- & 2 & -- & -- \\
\hline SEP & & & & & & & & & & & & \\
\hline 18. & -- & -- & 17 & $<0.1$ & -- & - & -- & 9 & -- & 2 & -- & -- \\
\hline
\end{tabular}


Table 34.--Chemical analyses of surface-water samples collected at Whitewood Creek above Whi tewood, S. Dak. --Cont inued

\section{WATER QUALITY DATA}

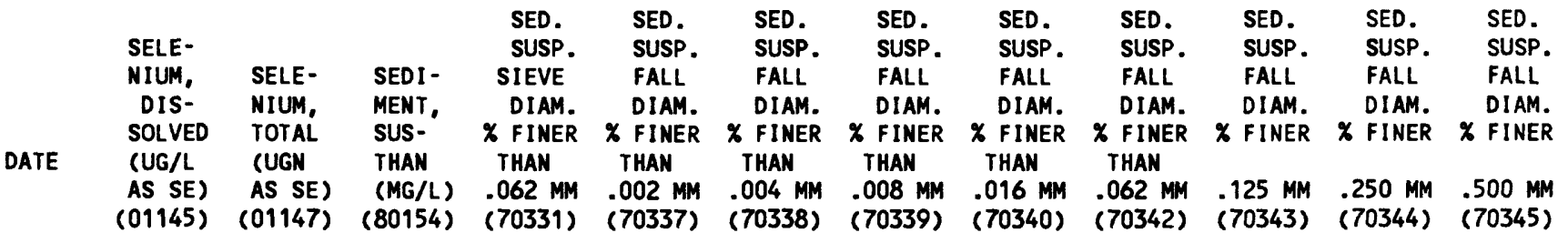

\section{FEB 1986}

$06 .$.

$21 \ldots$

$25 . .$.

26...

27...

28...

MAR

03... $\quad-.-12$

$05 . .$.

$10 .$.

APR

06...

23...

$28 .$.

MAY

$20 . .$.

JUN

04.

$09 .$.

$09 . .$.

$09 .$. .

$10 . .$.

$10 . .$.

$\begin{array}{ll}3 & \\ 3 & \\ 3 & - \\ -- & - \\ -- & - \\ -- & - \\ -- & - \\ -- & - \\ -- & -\end{array}$

\section{$3 \quad 10 \quad 85$}

JUL

AUG 17.

12 ...

SEP $\begin{array}{rrr}-- & -- & 27 \\ -- & -- & 213 \\ -- & -- & 34\end{array}$

$\begin{array}{llll}- & - & & 7\end{array}$

$\begin{array}{lll}-- & -- & 189\end{array}$

$\begin{array}{lll}- & - & \\ - & & 55\end{array}$

-. $\quad$-. 404

$\begin{array}{lll}- & - & \\ - & 728\end{array}$

$\begin{array}{lll}- & - & -\end{array}$

-. $\quad$-. 247

16

3

4
85

91

98

-.

$-\cdot$

98

97

88

96

$\begin{array}{lll}-- & - & - \\ -- & -- & -- \\ -- & -- & -- \\ -- & -- & -- \\ -- & -- & -- \\ -- & - & -\end{array}$

- $\quad-$

$+-$

$--\quad \quad-$

$--$

$-$

100

97

-. 31

.. $\quad 30$

98

88

66

90 
Table 35.--Chemical analyses of surface-water samples collected at Whitewood Creek above Vale, S. Dak.

WATER QUALITY DATA

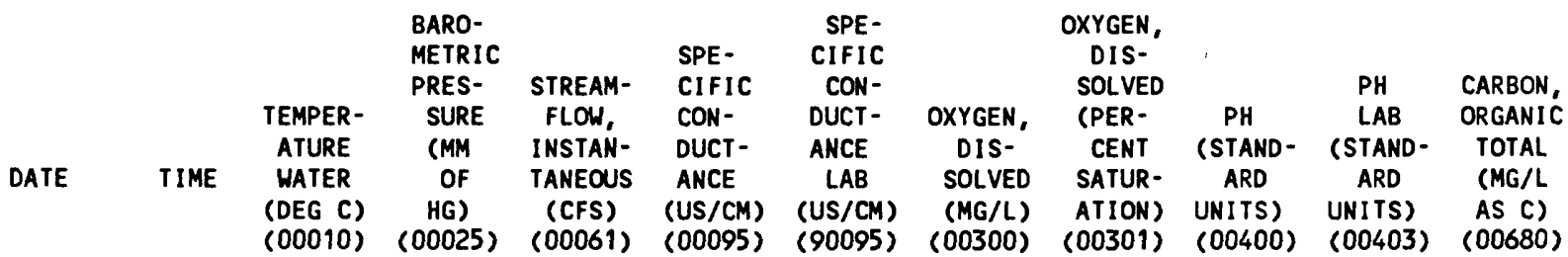

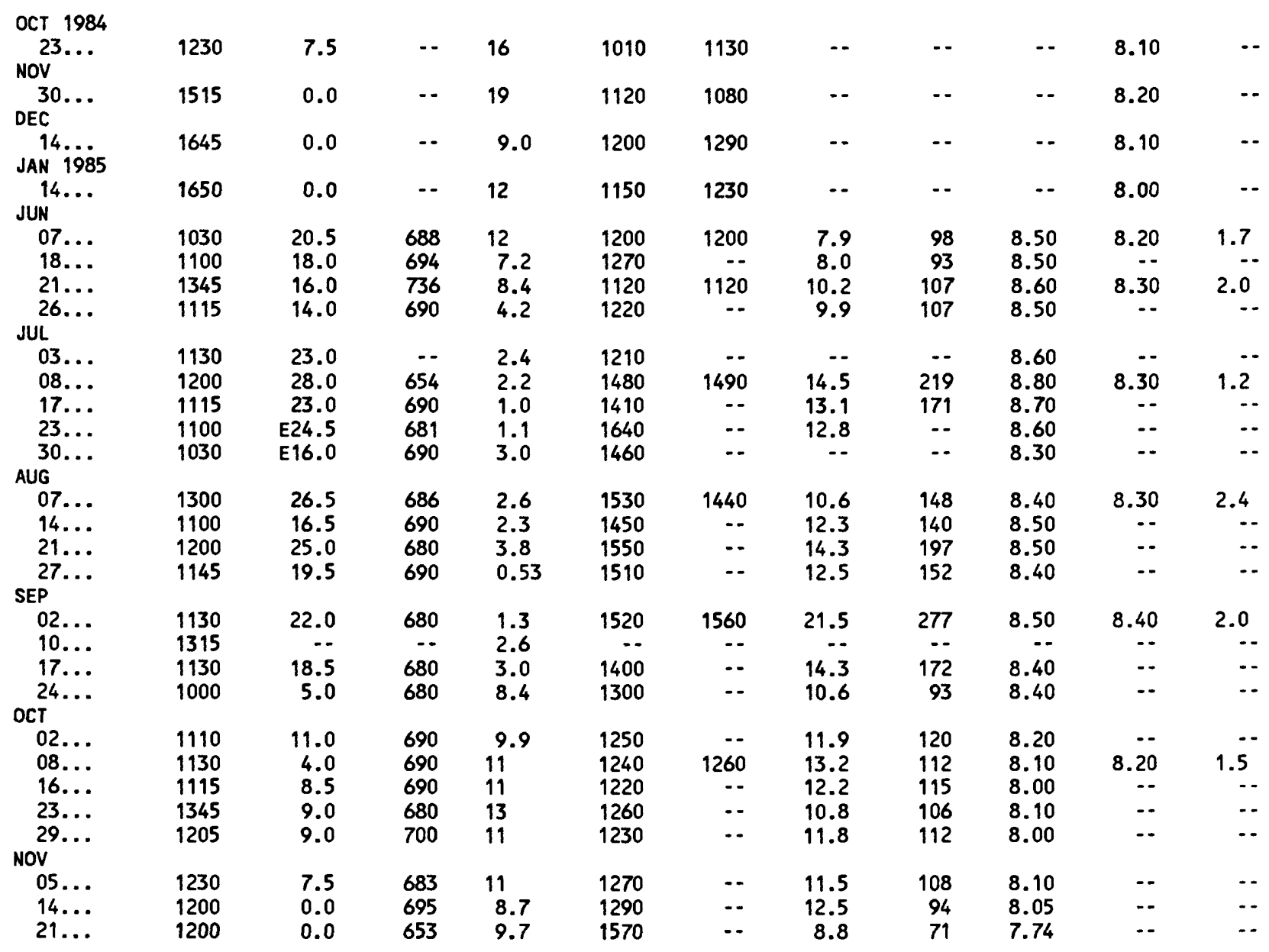


Table 35.--Chemical analyses of surface-water samples collected at Whitewood Creek above Vale, S. Dak.--Cont inued

WATER QUALITY DATA

\begin{tabular}{|c|c|c|c|c|c|c|c|c|c|c|c|c|}
\hline DATE & $\begin{array}{c}\text { CARBON, } \\
\text { ORGANIC } \\
\text { DIS- } \\
\text { SOLVED } \\
\text { (MG/L } \\
\text { AS C) } \\
(00681)\end{array}$ & $\begin{array}{c}\text { CYANIDE } \\
\text { TOTAL } \\
\text { (MG/L } \\
\text { AS CN) } \\
(00720)\end{array}$ & $\begin{array}{l}\text { CYANIDE } \\
\text { DIS- } \\
\text { SOLVED } \\
\text { (MG/L } \\
\text { AS CN) } \\
(00723)\end{array}$ & $\begin{array}{c}\text { SOLIDS, } \\
\text { RESIDUE } \\
\text { AT } 180 \\
\text { DEG. C } \\
\text { DIS- } \\
\text { SOLVED } \\
\text { (MG/L) } \\
(70300)\end{array}$ & $\begin{array}{l}\text { HARD- } \\
\text { NESS } \\
\text { (MG/L } \\
\text { AS } \\
\text { CACO3) } \\
(00900)\end{array}$ & $\begin{array}{c}\text { HARD- } \\
\text { NESS } \\
\text { NONCAR- } \\
\text { BONATE } \\
\text { (MG/L } \\
\text { AS } \\
\text { CACO3) } \\
(95902)\end{array}$ & $\begin{array}{c}\text { ALK } \\
\text { LINI } \\
\text { LAL } \\
\text { CMG } \\
\text { AS } \\
\text { CACC } \\
\text { (904 }\end{array}$ & & $\begin{array}{l}\text { CALCIUM } \\
\text { DIS- } \\
\text { SOLVED } \\
\text { (MG/L } \\
\text { AS CA) } \\
(00915)\end{array}$ & $\begin{array}{l}\text { MAGNE- } \\
\text { SIUM, } \\
\text { DIS- } \\
\text { SOLVED } \\
\text { (MG/L } \\
\text { AS MG) } \\
(00925)\end{array}$ & $\begin{array}{c}\text { SODIUM, } \\
\text { DIS- } \\
\text { SOLVED } \\
\text { (MG/L } \\
\text { AS NA) } \\
(00930)\end{array}$ & $\begin{array}{c}\text { SODIUM } \\
\text { AD- } \\
\text { SORP. } \\
\text { TION } \\
\text { RATIO } \\
(00931)\end{array}$ \\
\hline $\begin{array}{l}\text { CT } 1984 \\
23 \ldots \\
\text { IOV }\end{array}$ & -. & 0.010 & 0.02 & 848 & 550 & 380 & 168 & & 120 & 61 & 39 & 0.7 \\
\hline $\begin{array}{c}30 \ldots \\
D E C\end{array}$ & -. & 0.030 & 0.03 & 804 & 560 & 430 & 133 & & 130 & 57 & 37 & 0.7 \\
\hline $\begin{array}{l}14 \ldots \\
\text { JAN } 1985\end{array}$ & $\cdots$ & 0.020 & 0.02 & 984 & 690 & 510 & 182 & & 160 & 70 & 44 & 0.8 \\
\hline $\operatorname{JUN}^{14 \ldots}$ & -- & 0.030 & 0.03 & 924 & 650 & 450 & 199 & & 150 & 66 & 43 & 0.8 \\
\hline $07 \ldots$ & 4.0 & $<0.010$ & $<0.01$ & 894 & 600 & 460 & 140 & -- & 130 & $66 \ldots$ & 49 & 0.9 \\
\hline $\begin{array}{l}21 \ldots . \\
26 . .\end{array}$ & $30 \ldots$ & $\begin{array}{r}<0.010 \\
\ldots\end{array}$ & $<0.01$ & $\begin{array}{r}815 \\
\ldots .\end{array}$ & $\begin{array}{r}550 \\
\ldots-\end{array}$ & $\begin{array}{r}380 \\
\ldots\end{array}$ & 165 & $\ldots$ & 120 & $60 \ldots$ & $47^{-.}$ & 0.9 \\
\hline $\begin{array}{l}\text { JUL } \\
03 . . .\end{array}$ & -. & & $\cdots$ & $\cdots$ & $\cdots$ & $\cdots$ & & -. & -. & -. & -. & -- \\
\hline 08.. & 3.9 & $<0.010$ & 0.02 & 1170 & 770 & 630 & 144 & .. & 160 & $90 \ldots$ & $\begin{array}{ll}57 & \\
& \end{array}$ & 0.9 \\
\hline & -. & -. & .. & -. & .. & -. & & -. & -. & -- & -- & -. \\
\hline $\begin{array}{c}30 \\
\text { AUG }\end{array}$ & $\cdots$ & $\cdots$ & $\cdot-$ & $\cdot-$ & $\cdots$ & $\cdots$ & & $\cdot-$ & -. & -. & -- & $-\cdot$ \\
\hline $07 \ldots$ & 5.4 & $<0.010$ & $<0.01$ & 1150 & 740 & 600 & 136 & & 150 & 88 & 58 & 1 \\
\hline $\begin{array}{l}14 . \\
21 .\end{array}$ & -- & -- & $-\cdot$ & -- & $-\cdot$ & -- & & -- & - & -. & -- & -. \\
\hline $27 \ldots$ & .. & -- & - &.- &.- & -. & & - & -- & .. & -. & -. \\
\hline $\begin{array}{l}\text { SEP } \\
02\end{array}$ & 2,3 & $<0.010$ & $<0,01$ & $12<0$ & 890 & 670 & 137 & & 170 & Q & 60 & 00 \\
\hline & .. & -. & -. & .-. & 010 & or.- & 138 & -- & 110 & $\begin{array}{l}{ }^{44} \\
\end{array}$ & (0) & 0.9 \\
\hline & $\cdot-$ & $\cdot \cdot$ & $\cdots$ & $\cdots$ & -. & $\cdots$ & & -- & $\cdots$ & -- & $\cdots$ & $\cdot \cdot$ \\
\hline${ }_{\text {ост }}^{24 \ldots . .}$ & -- & -- & $\cdots$ & -- & $\cdots$ & $\cdots$ & & $-\cdot$ & -- & -. & $\cdots$ & -. \\
\hline 02. & -. & $\cdots$ & $\because$ & -- & -. & -- & & -- & -. & -. & .. & -. \\
\hline & 1.9 & 0.020 & 0.02 & 952 & 590 & 360 & 224 & & 130 & 64 & 48 & 0.9 \\
\hline & $\because$ & -- & $\ddot{--}$ & -. & -. & $\begin{array}{l}-- \\
--\end{array}$ & & 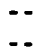 & $\because$ & -. & 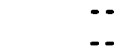 & -. \\
\hline & -- & -. & -- &.- & .. & -. & & -. & -- & .. & .- & -. \\
\hline Nov & & & & & & & & & & & & \\
\hline & $\because$ & -- & $\cdots$ & $-\cdot$ & 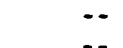 & -- & & -- & -- & $\because$ & -- & -- \\
\hline $\begin{array}{l}14 \ldots \\
21 \ldots\end{array}$ & -. & -. & - & $\ddot{-}$ & $\ldots$ & - & & $\because-$ & -. & $\therefore$ & -- & $\ddot{-.}$ \\
\hline
\end{tabular}


Table 35.---Chemical analyses of surface-water samples collected at Whitewood Creek above Vale, S. Dak.--Cont inued

WATER QUALITY DATA

\begin{tabular}{|c|c|c|c|c|c|c|c|c|c|c|c|}
\hline DATE & $\begin{array}{l}\text { PERCENT } \\
\text { SODIUM } \\
\text { (00932) }\end{array}$ & $\begin{array}{l}\text { POTAS- } \\
\text { SIUM, } \\
\text { DIS- } \\
\text { SOLVED } \\
\text { (MG/L } \\
\text { AS K) } \\
(00935)\end{array}$ & $\begin{array}{l}\text { POTAS- } \\
\text { SIUM, } \\
\text { TOTAL } \\
\text { RECOV- } \\
\text { ER/BLE } \\
\text { (MG/L } \\
\text { AS K) } \\
(00937)\end{array}$ & $\begin{array}{l}\text { CHLO- } \\
\text { RIDE, } \\
\text { DIS- } \\
\text { SOLVED } \\
\text { (MG/L } \\
\text { AS CL) } \\
(00940)\end{array}$ & $\begin{array}{l}\text { SULFATE } \\
\text { DIS- } \\
\text { SOLVED } \\
\text { (MG/L } \\
\text { AS SO4) } \\
\text { (00945) }\end{array}$ & $\begin{array}{l}\text { FLUO- } \\
\text { RIDE, } \\
\text { DIS- } \\
\text { SOLVED } \\
\text { (MG/L } \\
\text { AS F) } \\
(00950)\end{array}$ & $\begin{array}{l}\text { ARSENIC } \\
\text { DIS- } \\
\text { SOLVED } \\
\text { (UG/L } \\
\text { AS AS) } \\
\text { (01000) }\end{array}$ & $\begin{array}{c}\text { ARSENIC } \\
\text { SUS- } \\
\text { PENDED } \\
\text { TOTAL } \\
\text { (UG/L } \\
\text { AS AS) } \\
\text { (01001) }\end{array}$ & $\begin{array}{c}\text { ARSENIC } \\
\text { TOTAL } \\
\text { (UG/L } \\
\text { AS AS) } \\
(01002)\end{array}$ & $\begin{array}{l}\text { BORON, } \\
\text { DIS- } \\
\text { SOLVED } \\
\text { (UG/L } \\
\text { AS B) } \\
(01020)\end{array}$ & $\begin{array}{l}\text { BORON, } \\
\text { TOTAL } \\
\text { RECOV- } \\
\text { ERABLE } \\
\text { (UG/L } \\
\text { AS B) } \\
(01022)\end{array}$ \\
\hline
\end{tabular}

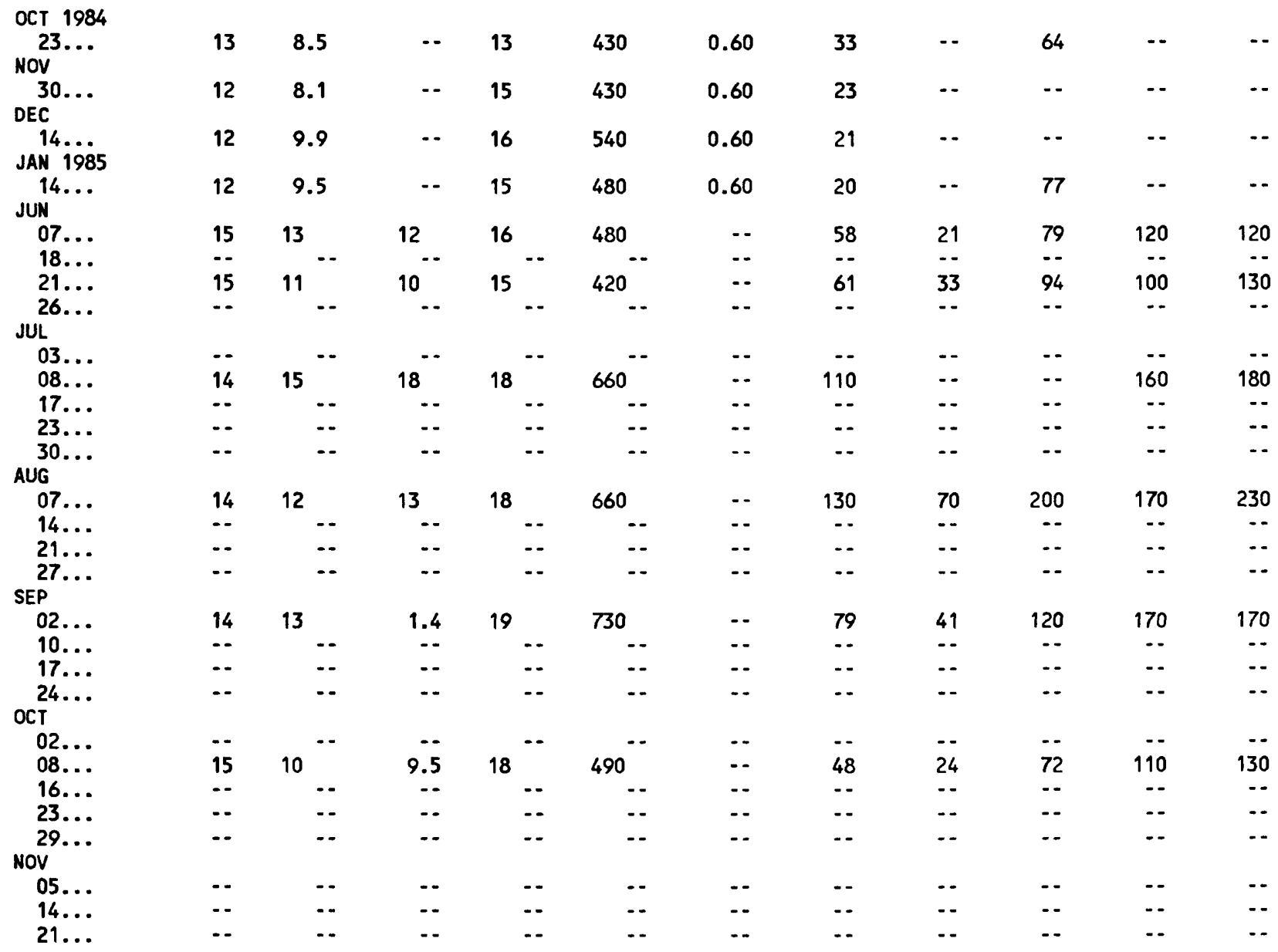


Table 35.--Chemical analyses of surface-water samples collected at Whitewood Creek above Vale, S. Dak.--Cont inued

WATER QUALITY DATA

\begin{tabular}{|c|c|c|c|c|c|c|c|c|c|c|c|}
\hline ATE & $\begin{array}{l}\text { CADMIUM } \\
\text { DIS- } \\
\text { SOLVED } \\
\text { (UG/L } \\
\text { AS CD) } \\
(01025)\end{array}$ & $\begin{array}{l}\text { CADMIUM } \\
\text { TOTAL } \\
\text { RECOV- } \\
\text { ERABLE } \\
\text { (UG/L } \\
\text { AS CD) } \\
(01027)\end{array}$ & $\begin{array}{l}\text { CHRO- } \\
\text { MIUM, } \\
\text { DIS- } \\
\text { SOLVED } \\
\text { (UG/L } \\
\text { AS CR) } \\
(01030)\end{array}$ & $\begin{array}{l}\text { CHRO- } \\
\text { MIUM, } \\
\text { TOTAL } \\
\text { RECOV- } \\
\text { ERABLE } \\
\text { (UG/L } \\
\text { AS CR) } \\
(01034)\end{array}$ & $\begin{array}{l}\text { COPPER, } \\
\text { DIS- } \\
\text { SOLVED } \\
\text { (UG/L } \\
\text { AS CU) } \\
(01040)\end{array}$ & $\begin{array}{l}\text { COPPER, } \\
\text { SUS- } \\
\text { PENDED } \\
\text { RECOV- } \\
\text { ERABLE } \\
\text { (UG/L } \\
\text { AS CU) } \\
(01041)\end{array}$ & $\begin{array}{l}\text { COPPER, } \\
\text { TOTAL } \\
\text { RECOV- } \\
\text { ERABLE } \\
\text { (UG/L } \\
\text { AS CU) } \\
\text { (01042) }\end{array}$ & $\begin{array}{l}\text { IRON, } \\
\text { SUS- } \\
\text { PENDED } \\
\text { RECOV- } \\
\text { ERABLE } \\
\text { (UG/L } \\
\text { AS FE) } \\
(01044)\end{array}$ & $\begin{array}{l}\text { IRON, } \\
\text { TOTAL } \\
\text { RECOV- } \\
\text { ERABLE } \\
\text { (UG/L } \\
\text { AS FE) } \\
\text { (01045) }\end{array}$ & $\begin{array}{l}\text { IRON, } \\
\text { DIS- } \\
\text { SOLVED } \\
\text { (UG/L } \\
\text { AS FE) } \\
(01046)\end{array}$ & $\begin{array}{l}\text { LEAD, } \\
\text { DIS- } \\
\text { SOLVED } \\
\text { (UG/L } \\
\text { AS PB) } \\
(01049)\end{array}$ \\
\hline
\end{tabular}

\begin{tabular}{|c|c|c|c|c|c|c|c|c|c|c|c|}
\hline $\begin{array}{l}\text { OCT } 1984 \\
23 . . \\
\text { NoV }\end{array}$ & 1 & $<10$ & $\cdots$ & -- & $<10$ & $\cdots$ & 10 & $\cdots$ & 580 & 12 & $\cdots$ \\
\hline DEC & $<1$ & $<10$ & -- & $\cdots$ & $<10$ & $\cdots$ & 20 & $\cdots$ & 1200 & 11 & $\cdots$ \\
\hline JAN $14 \ldots 85$ & $<1$ & $<10$ & $\cdots$ & $\cdots$ & $<10$ & $\cdots$ & 20 & $\cdots$ & 830 & 14 & $\cdots$ \\
\hline $\begin{array}{l}14 \ldots \\
\text { JUN }\end{array}$ & $<1$ & $<10$ & $\cdots$ & $\cdots$ & $<10$ & - & 60 & $\cdots$ & 850 & 16 & $\cdots$ \\
\hline $07 \ldots$ & $<1$ & $<1$ & $<10$ & $<10$ & 2 & 1 & 3 & 370 & 380 & 8 & 3 \\
\hline $\begin{array}{l}18 \ldots \\
21 \ldots\end{array}$ & $\begin{array}{l}-- \\
<1\end{array}$ & $\ddot{<1}$ & $<-=$ & $\overline{20}$ & -- & -- & -- & 660 & 670 & $\ddot{10}$ & -8 \\
\hline $26 \ldots$ & $\therefore$ & $\therefore$ & -. & $\therefore$ & $\therefore$ & $\therefore$ & $\therefore$ & -. & - & $\therefore$ & $\cdots$ \\
\hline JUL & & & & & & & & & & & \\
\hline $03 \ldots$ & $\cdots$ & $\cdots$ & $\cdots$ & $\cdots$ & $\cdots$ & $\cdots$ & $\cdots$ & -. & $\cdots$ & $\cdots$ & $\cdots$ \\
\hline $08 \ldots$ & $<1$ & $<1$ & $<10$ & $<10$ & 2 & 0 & 2 & 730 & 740 & 6 & $<1$ \\
\hline $17 \ldots$ & -- & -- & -. & -. & -- & $-\cdot$ & $-\cdot$ & -. & $\cdots$ & $\cdots$ & $\cdots$ \\
\hline $23 \ldots$ & $\cdots$ & $\cdots$ & $\cdots$ & -- & -- & $\cdots$ & $\cdots$ & $\cdots$ & $\cdots$ & $\cdots$ & $\cdots$ \\
\hline $30 \ldots$ & $\cdots$ & $\cdots$ & $\cdots$ & -- & $-\cdot$ & -- & -- & $\cdots$ & $\cdots$ & $\cdots$ & -- \\
\hline AUG & & & & & & & & & & & \\
\hline $07 \ldots$ & $<1$ & $<1$ & $<10$ & $<10$ & 1 & 1 & 2 & 470 & 480 & 10 & $<1$ \\
\hline $14 \ldots$ & -- & $\cdots$ & 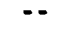 & -- & -- & -- & $-\cdot$ & -- & $-\cdot$ & -- & - \\
\hline $21 \ldots$ & -- & $-\cdot$ & $\cdots$ & $\cdots$ & $\cdots$ & $\cdots$ & $\cdots$ & $\cdots$ & $\cdots$ & $\cdots$ & $\cdots$ \\
\hline $27 \ldots$ & $\cdots$ & $\cdots$ & $\cdots$ & $\cdots$ & $\cdots$ & -- & $\cdots$ & $\cdots$ & $\cdots$ & -- & $\cdots$ \\
\hline SEP & & & & & & & & & & & \\
\hline $02 \ldots$ & $<1$ & $<1$ & $<10$ & $<10$ & $<1$ & $\cdots$ & 2 & 400 & 410 & 7 & $<1$ \\
\hline $10 \ldots$ & $\cdots$ & $\cdots$ & $\cdots$ & -- & $\cdots$ & $\cdots$ & $\cdots$ & -- & -- & -- & -- \\
\hline $17 \ldots$ & $\cdots$ & $\cdots$ & $\because$ & $-\cdot$ & $\cdots$ & -- & $\cdots$ & -- & -- & -- & $\cdots$ \\
\hline $24 \ldots$ & $\cdots$ & $\cdots$ & $\cdots$ & $\cdots$ & $\cdots$ & $\cdots$ & $\cdots$ & $\cdots$ & $\cdots$ & $\cdots$ & -- \\
\hline $02 \ldots$ & $\cdots$ & $\cdots$ & -- & -- & -- & -- & -- & $-\cdot$ & $\cdots$ & $\cdots$ & $\cdots$ \\
\hline $08 \ldots$ & $<1$ & $<1$ & $<10$ & $<10$ & 2 & 0 & 2 & 330 & 340 & 11 & 1 \\
\hline $16 \ldots$ & $\cdots$ & $\cdots$ & - & -. & $\cdots$ & $\cdots$ & -- & $\cdots$ & $\cdots$ & $\cdots$ & $\cdots$ \\
\hline $23 \ldots$ & $\cdots$ & $\cdots$ & $\cdots$ & $\cdots$ & $\cdots$ & $\cdots$ & $-\cdot$ & $\cdots$ & $\cdots$ & $\cdots$ & $\cdots$ \\
\hline $29 .$. & $\cdots$ & $\cdots$ & -- & -- & $\cdots$ & -- & $\cdots$ & -- & $\cdots$ & $\cdots$ & -- \\
\hline NOV & & & & & & & & & & & \\
\hline $05 \ldots$ & $\because$ & -- & $-\cdot$ & $\because$ & $\cdots$ & -- & $\cdots$ & $\cdots$ & -- & $\cdots$ & $\cdots$ \\
\hline $14 \ldots$ & $\cdots$ & $\cdots$ & $\cdots$ & $\cdots$ & $\cdots$ & $\cdots$ & -- & $\cdots$ & $\cdots$ & $\cdots$ & $\cdots$ \\
\hline $21 \ldots$ & -- & $\cdots$ & -- & -- & -- & -- & -- & -- & $\cdots$ & -- & $\cdots$ \\
\hline
\end{tabular}


Table 35.--Chemical analyses of surface-water samples collected at Whitewood Creek above Vale, S. Dak.--Cont inued

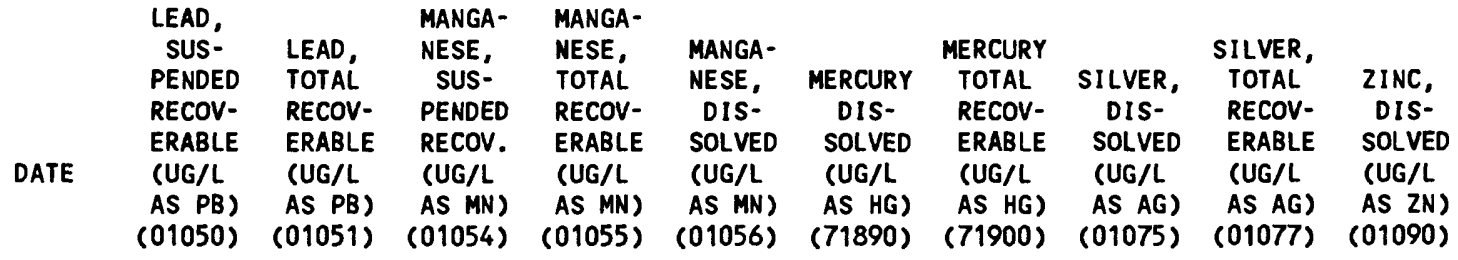

\begin{tabular}{|c|c|c|c|c|c|c|c|c|c|c|}
\hline $\begin{array}{c}\text { OCT } 1984 \\
23 . . . \\
\text { NOV }\end{array}$ & -- & $\cdots$ & $\cdot-$ & 190 & 170 & $<0.1$ & $<0.10$ & $<1$ & 1 & $\cdots$ \\
\hline DEC $30 .$. & -- & -- & -- & 210 & 170 & $<0.1$ & $<0.10$ & $<1$ & $<1$ & $\cdots$ \\
\hline $\begin{array}{c}14 \ldots \\
\text { JAN } 1985\end{array}$ & -- & -- & -- & 310 & 300 & $<0.1$ & $<0.10$ & $<1$ & $<1$ & $\cdots$ \\
\hline $\begin{array}{c}14 \ldots \\
\text { JUN }\end{array}$ & -- & $\cdots$ & $\cdots$ & 230 & 220 & $<0.1$ & 0.10 & $<1$ & $<1$ & $\cdots$ \\
\hline $07 .$. & 0 & 1 & 20 & 90 & 70 & $<0.1$ & $<0.10$ & $<1$ & $<1$ & $<3$ \\
\hline $18 \ldots$ & $\overline{0}$ & $\because$ & $\ddot{70}$ & $\ddot{i n}$ & $\ddot{15}$ & $=$ & 0 & $\because$ & $\because$ & $\overline{5}$ \\
\hline $21 \ldots$ & 0 & 2 & 70 & 110 & 45 & $<0.1$ & 0.10 & $<1$ & $<1$ & 5 \\
\hline $26 \ldots$ & -- & $\cdots$ & $\cdots$ & -- & $\cdots$ & -- & -- & $\cdots$ & $\cdots$ & -- \\
\hline JUL & & & & & & & & & & \\
\hline $03 . .$. & -- & $\because$ & $\because$ & $\cdots$ & -- & -- & -- & $\cdots$ & $\because$ & $\cdots$ \\
\hline $08 . .$. & -- & $<1$ & 20 & 160 & 140 & $<0.1$ & $<0.10$ & $<1$ & $<1$ & 4 \\
\hline $17 \ldots$ & -- & $\cdots$ & -- & -- & -- & -- & -- & $\cdots$ & -- & -- \\
\hline $23 \ldots$ & -- & -- & -- & -- & $\cdots$ & $\cdots$ & -- & -- & $\cdots$ & -- \\
\hline $30 \ldots$ & -- & -- & -- & -- & $\cdots$ & $\cdots$ & $\cdots$ & $\cdots$ & $-\cdot$ & $\cdots$ \\
\hline AUG & & & & & & & & & & \\
\hline $07 \ldots$ & -- & 1 & 20 & 60 & 41 & $<0.1$ & $<0.10$ & $<1$ & $<1$ & 7 \\
\hline $14 \ldots$ & $\because-$ & $\cdots$ & $\cdots$ & $\cdots$ & $\cdots$ & $\cdots$ & $\cdots$ & $\cdots$ & $\because$ & $\cdots$ \\
\hline $21 \ldots$ & 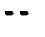 & $\cdots$ & $-\cdot$ & $\cdots$ & $\cdots$ & $\cdots$ & $\cdots$ & -- & $-\cdot$ & -- \\
\hline $27 \ldots$ & -- & -- & -- & $\cdots$ & $\cdots$ & $\cdots$ & -- & $\cdots$ & -- & -- \\
\hline SEP & & & & & & & & & & \\
\hline $02 \ldots$ & -- & 1 & 10 & 80 & 66 & $<0.1$ & $<0.10$ & 1 & 1 & 13 \\
\hline $10 \ldots$ & -- & $\cdots$ & -- & $\cdots$ & $\because$ & -- & $\cdots$ & $\cdots$ & $\cdots$ & $\cdots$ \\
\hline $17 \ldots$ & $\cdots$ & -- & -- & -- & -- & $\cdots$ & $\cdots$ & -- & $\cdots$ & $\cdots$ \\
\hline $24 \ldots$ & -- & $\because$ & $\cdots$ & -- & $\cdots$ & $\cdots$ & -- & -- & -- & -- \\
\hline OCT & & & & & & & & & & \\
\hline $02 \ldots$ & -- & $\because$ & -- & -- & $\because$ & -- & $\cdots$ & -- & $\cdots$ & -- \\
\hline $08 \ldots$ & -- & $<1$ & 20 & 120 & 99 & 0.3 & 0.30 & $<1$ & $<1$ & 9 \\
\hline $16 \ldots$ & $\because$ & $\cdots$ & -- & -- & $-\cdot$ & $\cdots$ & -- & -- & -- & $\cdots$ \\
\hline $23 \ldots$ & $\cdots$ & $\cdots$ & $\cdots$ & $\cdots$ & $\cdots$ & $\cdots$ & $\cdots$ & -- & $\cdots$ & $\cdots$ \\
\hline $29 \ldots$ & $-\cdot$ & $\cdots$ & $\cdots$ & -- & $\cdots$ & -- & $\cdots$ & $\cdots$ & $\cdots$ & $\cdots$ \\
\hline NOV & & & & & & & & & & \\
\hline $05 \ldots$ & -- & $\cdots$ & -- & -- & $\cdots$ & $\cdots$ & $\cdots$ & $\cdots$ & -- & $\cdots$ \\
\hline $14 \ldots$ & -- & $\cdots$ & -- & -- & $\cdots$ & -- &.- & -- & $\cdots$ & $=-$ \\
\hline $21 \ldots$ & -- & $\cdots$ & $\cdots$ & -- & $\therefore$ & -- & $\cdots$ & -- & -- & - \\
\hline
\end{tabular}


Table 35. - - Chemical analyses of surface-water samples collected at Whitewood Creek above Vale, S. Dak.--Cont inued

WATER QUALITY DATA

\begin{tabular}{|c|c|c|c|c|c|c|c|c|c|c|}
\hline ATE & $\begin{array}{l}\text { ZINC, } \\
\text { SUS- } \\
\text { PENDED } \\
\text { RECOV- } \\
\text { ERABLE } \\
\text { (UG/L } \\
\text { AS ZN) } \\
(01091)\end{array}$ & $\begin{array}{l}\text { ZINC, } \\
\text { TOTAL } \\
\text { RECOV- } \\
\text { ERABLE } \\
\text { (UG/L } \\
\text { AS ZN) } \\
\text { (01092) }\end{array}$ & $\begin{array}{l}\text { ALUM- } \\
\text { INUM, } \\
\text { TOTAL } \\
\text { RECOV- } \\
\text { ERABLE } \\
\text { (UG/L } \\
\text { AS AL) } \\
(01105)\end{array}$ & $\begin{array}{l}\text { ALUM- } \\
\text { INUM, } \\
\text { DIS- } \\
\text { SOLVED } \\
\text { (UG/L } \\
\text { AS AL) } \\
(01106)\end{array}$ & $\begin{array}{l}\text { ALUM- } \\
\text { INUM, } \\
\text { SUS- } \\
\text { PENDED } \\
\text { RECOV. } \\
\text { (UG/L } \\
\text { AS AL) } \\
(01107)\end{array}$ & $\begin{array}{l}\text { SELE- } \\
\text { NIUM, } \\
\text { DIS- } \\
\text { SOLVED } \\
\text { (UG/L } \\
\text { AS SE) } \\
\text { (01145) }\end{array}$ & $\begin{array}{l}\text { SELE- } \\
\text { NIUM, } \\
\text { SUS- } \\
\text { PENDED } \\
\text { TOTAL } \\
\text { (UG/L } \\
\text { AS SE) } \\
(01146)\end{array}$ & $\begin{array}{l}\text { SELE- } \\
\text { NIUM, } \\
\text { TOTAL } \\
\text { (UG/L } \\
\text { AS SE) } \\
(01147)\end{array}$ & $\begin{array}{l}\text { SEDI - } \\
\text { MENT, } \\
\text { SUS- } \\
\text { PENDED } \\
(M G / L) \\
(80154)\end{array}$ & $\begin{array}{c}\text { SED. } \\
\text { SUSP. } \\
\text { SIEVE } \\
\text { DIAM. } \\
\% \text { FINER } \\
\text { THAN } \\
.062 \text { MM } \\
(70331)\end{array}$ \\
\hline
\end{tabular}

OCT 1984

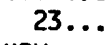

NOV

DEC 30.

$14 \ldots$

JAN 1985

$14 \ldots$

JUN

$07 . .$.

$18 . .$.

21...

$26 . .$.

JUL

$03 .$.

$08 . .$.

$17 . .$.

23...

$30 .$.

AUG

$07 .$. .

$14 \ldots$

$21 \ldots$

$27 . .$.

SEP

$02 . .$.

$10 \ldots$

$17 . .$.

$24 \ldots$

OCT

$02 . .$.

$08 .$.

$16 . .$.

23...

29.

NOV

$05 . .$.

$14 \ldots$

\begin{tabular}{|c|c|}
\hline-- & $\cdots$ \\
\hline-- & -- \\
\hline - & $\cdots$ \\
\hline$\cdots$ & $\cdots$ \\
\hline$\cdots$ & 40 \\
\hline - & -- \\
\hline 5 & 10 \\
\hline$\cdots$ & -- \\
\hline$\cdots$ & $\cdots$ \\
\hline 30 & 30 \\
\hline-- & $\cdots$ \\
\hline-- & - \\
\hline$\cdots$ & $\cdots$ \\
\hline - & $<10$ \\
\hline & - \\
\hline & -- \\
\hline- & $\cdots$ \\
\hline
\end{tabular}
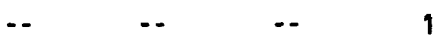

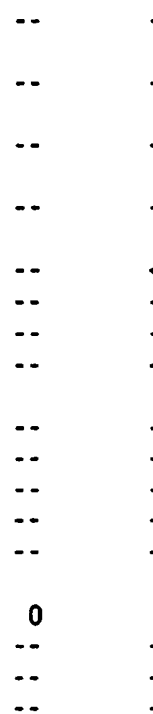

-. $\quad 21$

$21 \quad 47$

$\begin{array}{lll}- & - & -\end{array}$

$--53$

78

-

-. 20

62

$\begin{array}{lll}- & - & -1 \\ - & --\end{array}$

$\begin{array}{lll}- & 19 & 65\end{array}$

$21 . .$.

\begin{tabular}{|c|c|}
\hline 0 & 10 \\
\hline$\cdots$ & $\cdots$ \\
\hline-- & -- \\
\hline - & - \\
\hline 1 & 10 \\
\hline- & - \\
\hline - & - \\
\hline$\therefore$ & $\cdots$ \\
\hline -. & $\cdots$ \\
\hline - & . \\
\hline - & $\ldots$ \\
\hline
\end{tabular}

20

0

$<1$

$\cdots$

$\begin{array}{ll}4 & 94 \\ 4 & 79\end{array}$

$\begin{array}{lll}- & 29 & 97\end{array}$

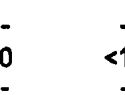

$\begin{array}{rrrrrrr}- & - & - & \ldots & \ldots & 75 & 97 \\ 10 & -. & 2 & \ldots & \ldots & 5 & 79\end{array}$

$\begin{array}{lll}-- & 5 & 90\end{array}$

$\begin{array}{lll}- & 4 & 87\end{array}$

$--$

1
--
--

$\begin{array}{ll}3 & 76 \\ 4 & 76 \\ 2 & 87 \\ 4 & 86\end{array}$

0

$\begin{array}{ll}5 & \\ -- & - \\ -- & -\end{array}$

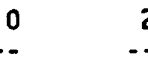

292

-.

$--$

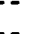

$\because$

$\begin{array}{rrrrr}- & - & - & - & - \\ 20 & 10 & 2 & 0 & 2\end{array}$

-

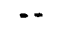

-.

$-$

$\ddot{-.}$

$\because$

$\begin{array}{lll}-. & -. & \\ & -.\end{array}$

$\cdot$

$-$

$\begin{array}{lll}-. & -. & - \\ -- & -- & -- \\ -. & -- & -\end{array}$

--

$\begin{array}{rr}-- & 1 \\ -- & 40 \\ -- & 114\end{array}$

100

60 
Table 35.--Chemical analyses of surface-water samples collected at Whitewood Creek above Vale, S. Dak.--Cont inued

WATER QUALITY DATA

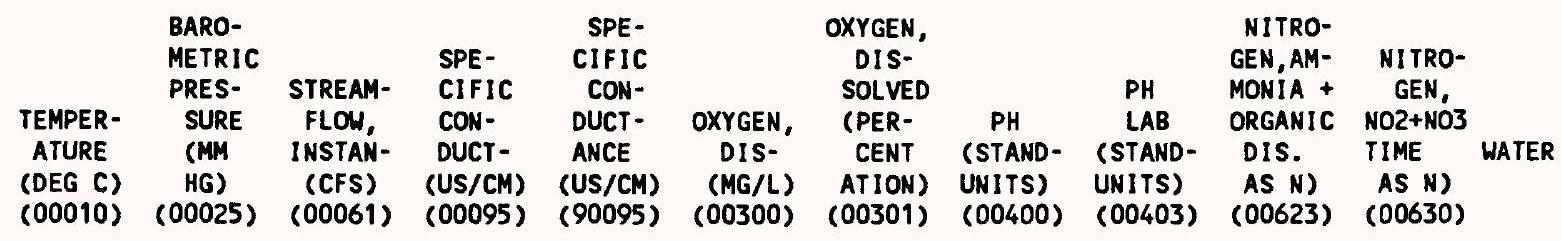

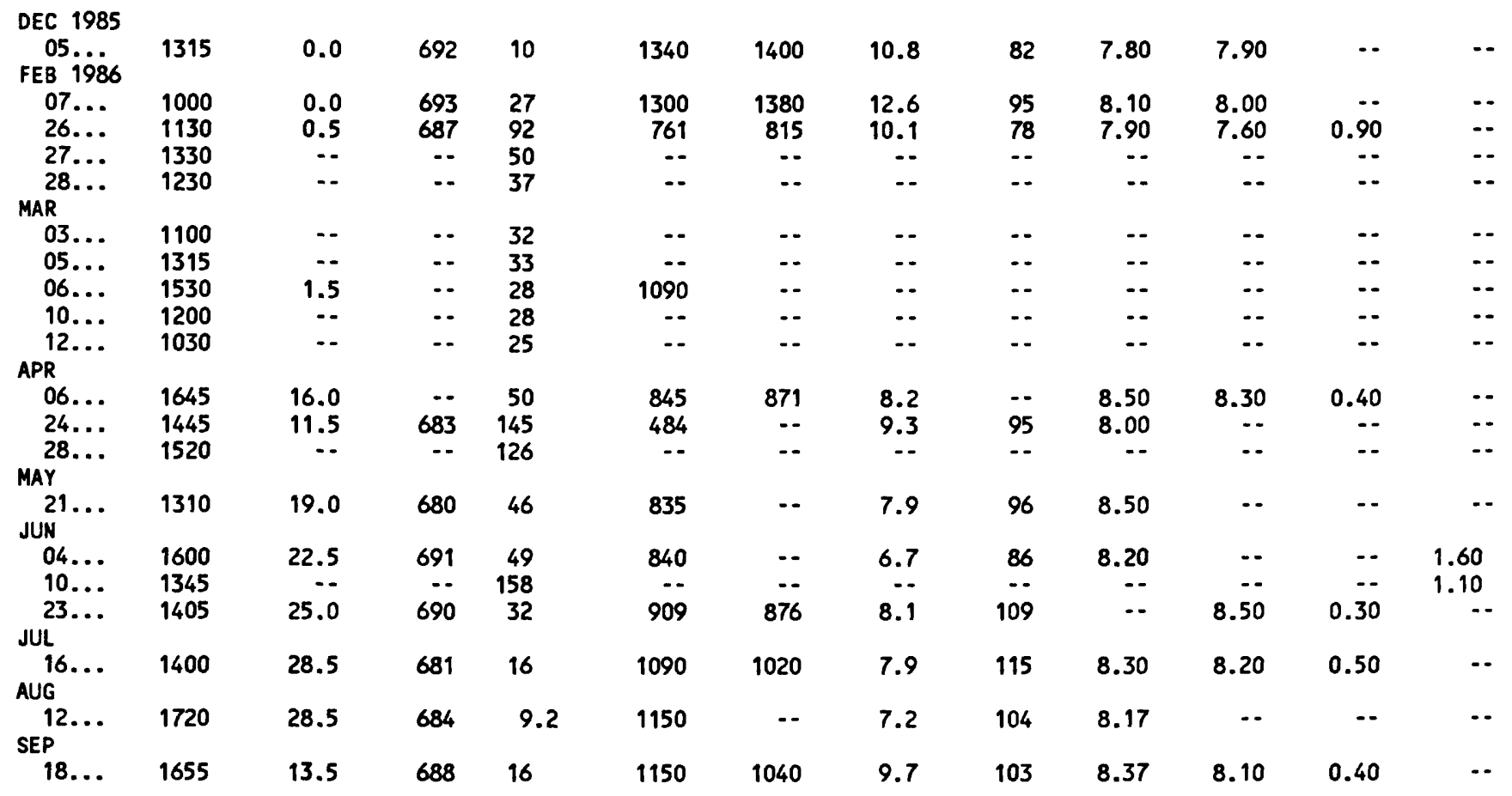


Table 35.--Chemical analyses of surface-water samples collected at whitewood Creek above Vale, S. Dak.--Cont inued

WATER QUALITY DATA

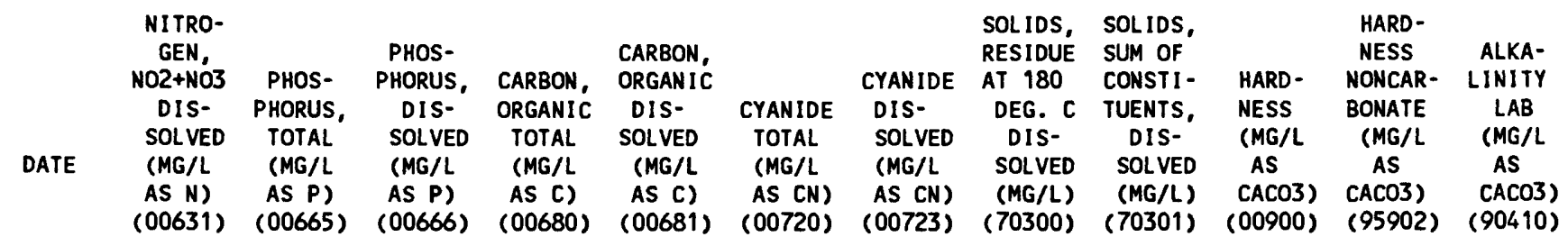

\begin{tabular}{|c|c|c|c|c|c|c|c|c|c|c|c|c|}
\hline $\begin{array}{c}\text { DEC } 1985 \\
05 \ldots \\
\text { FEB } 1986\end{array}$ & $\cdots$ & -- & -- & 3.0 & 2.0 & 0.020 & 0.02 & 525 & $\cdots$ & 690 & 480 & 207 \\
\hline $07 \ldots$ & $\ddot{2}$ & $\cdots$ & 0 & 3.4 & 3.1 & 0.020 & 0.02 & $\begin{array}{r}1040 \\
558\end{array}$ & -- & 650 & 460 & 192 \\
\hline $26 \ldots$ & 2.50 & -- & 0.040 & -- & -. & -- & 0.02 & 558 & $\cdots$ & $\cdots$ & $\cdots$ & 104 \\
\hline & - & -- & -. & -- & -- & -- & -- & $-\cdot$ & -- & $\cdots$ & -- & \\
\hline 28... & $\cdots$ & $\cdots$ & $\cdots$ & $\cdots$ & $\cdots$ & $\cdots$ & -- & $\cdots$ & -- & $\cdots$ & $\cdots$ & \\
\hline $03 .$. & - & -. & -. & - & & & & & & & & \\
\hline $05 \ldots$ & -. & -- & .. & $\ldots$ & $\ldots$ & -- & $\cdots$ & - & -- & $\cdots$ & $\ldots$ & \\
\hline $06 \ldots$ & $\cdots$ & -. & -. & .. & - & $\ldots$ & $\ldots$ & -. & $\ldots$ & .- & .. & \\
\hline $10 \ldots$ & $\cdots$ & $\cdots$ & - & $\cdots$ & - & -. & -- & $\cdots$ & -- & -- & .. & \\
\hline $12 \ldots$ & -- & -- & -- & $\cdots$ & -- & -- & -- & - & -. & $-\cdot$ & -. & \\
\hline APR & & & & & & & & & & & & \\
\hline $06 .$. & 2.00 & $\cdots$ & 0.023 & 7.8 & 3.4 & $\cdots$ & 0.02 & 611 & 580 & 380 & 240 & 136 \\
\hline $24 \ldots$ & -- & -- & - & 12 & -. & $\cdots$ & -- & -- & -- & $\cdots$ & -- & \\
\hline $28 .$. & -- & -- & -- & -- & -- & -- & $\cdots$ & -- & -- & $\cdots$ & $\cdots$ & \\
\hline MAY & & & & & & & & & & & & \\
\hline$\underset{\text { JUN }}{21 . . .}$ & -- & $\cdots$ & -- & -- & -- & -- & -- & $\cdots$ & $\cdots$ & -- & $\cdots$ & \\
\hline $04 \ldots$ & -- & 0.210 & $\cdots$ & 9.0 & $\cdots$ & $\cdots$ & $\cdots$ & -- & $\cdots$ & $-\cdot$ & $\cdots$ & \\
\hline $10 \ldots$ & -- & 2.10 & -- & 40 & -- & -- & $\cdots$ & -- & $\cdots$ & $\cdots$ & $\cdots$ & \\
\hline $23 \ldots$ & 1.70 & -- & 0.020 & 2.2 & 2.3 & -- & $<0.01$ & 658 & 620 & 440 & 260 & 174 \\
\hline JUL & & & & & & & & & & & & \\
\hline${ }_{A U G}^{16 \ldots}$ & 1.80 & -- & 0.153 & 1.9 & 2.0 & $\cdots$ & 0.01 & 815 & 790 & 540 & 390 & 155 \\
\hline $12 .$. & $\cdots$ & $\cdots$ & -- & -- & -- & -- & $\ldots$ & -- & -- & -- & $\cdots$ & \\
\hline SEP & & & & & & & & & & & & \\
\hline $18 \ldots$ & 3.20 & $\cdots$ & 0.027 & 2.1 & 1.7 & $-\cdot$ & 0.01 & 940 & 800 & 540 & 400 & 139 \\
\hline
\end{tabular}


Table 35.--Chemical analyses of surface-water samples collected at Whi tewood Creek above Vale, S. Dak. --Cont inued

WATER QUALITY DATA

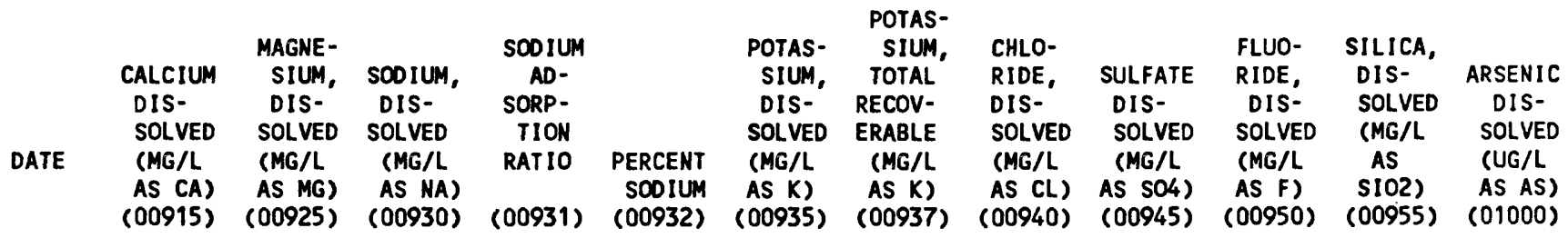

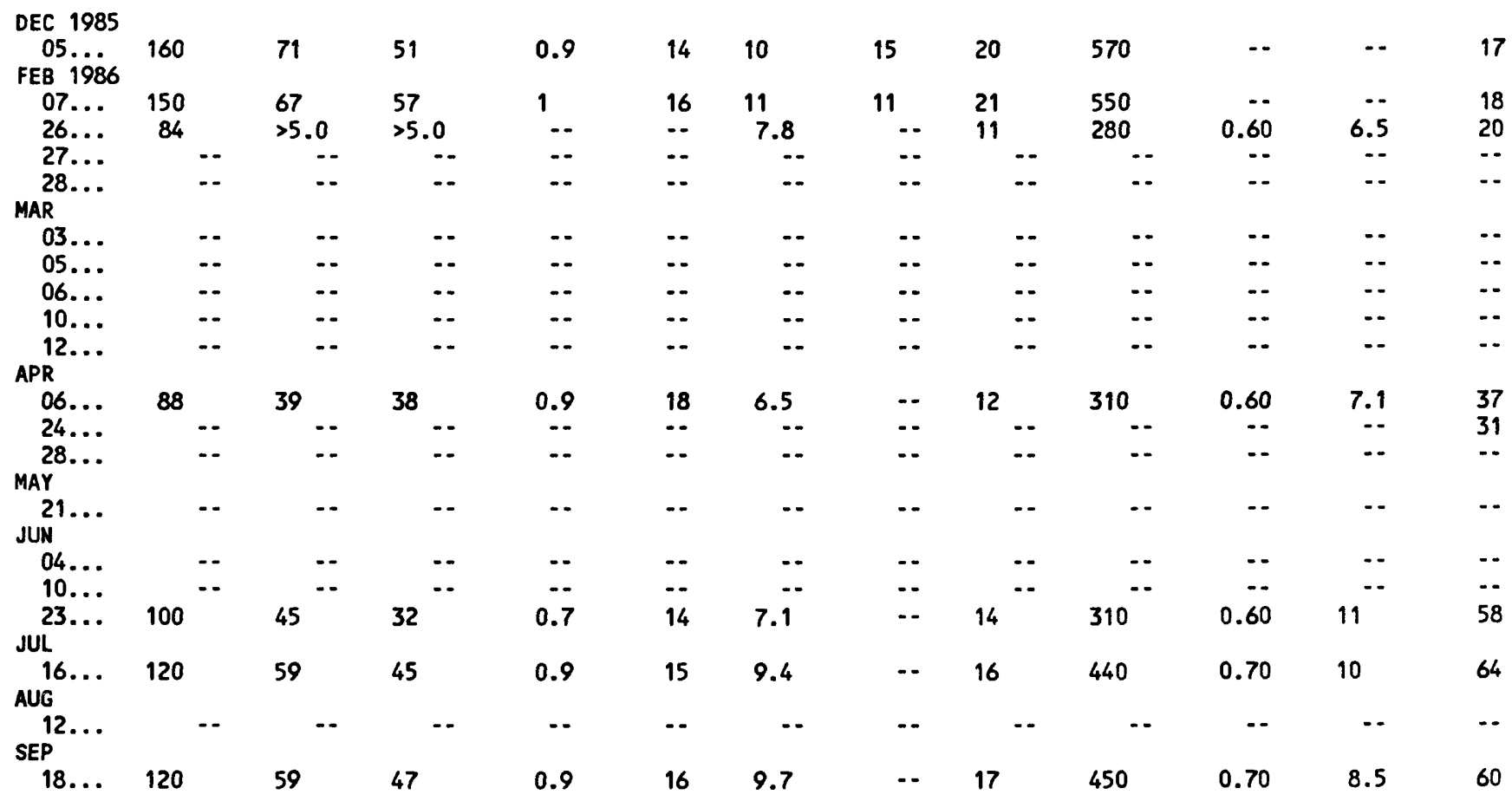


Table 35.--Chemical analyses of surface-water samples collected at Whitewood Creek above Vale, S. Dak. --Cont inued

\section{WATER QUALITY DATA}

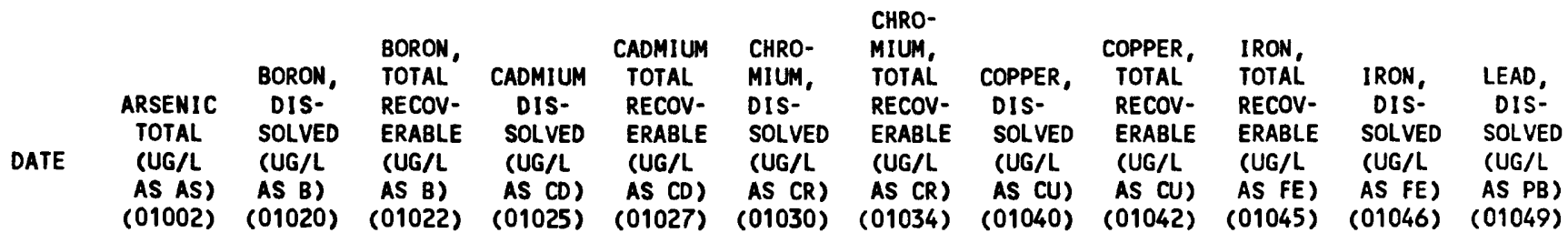

DEC 1985

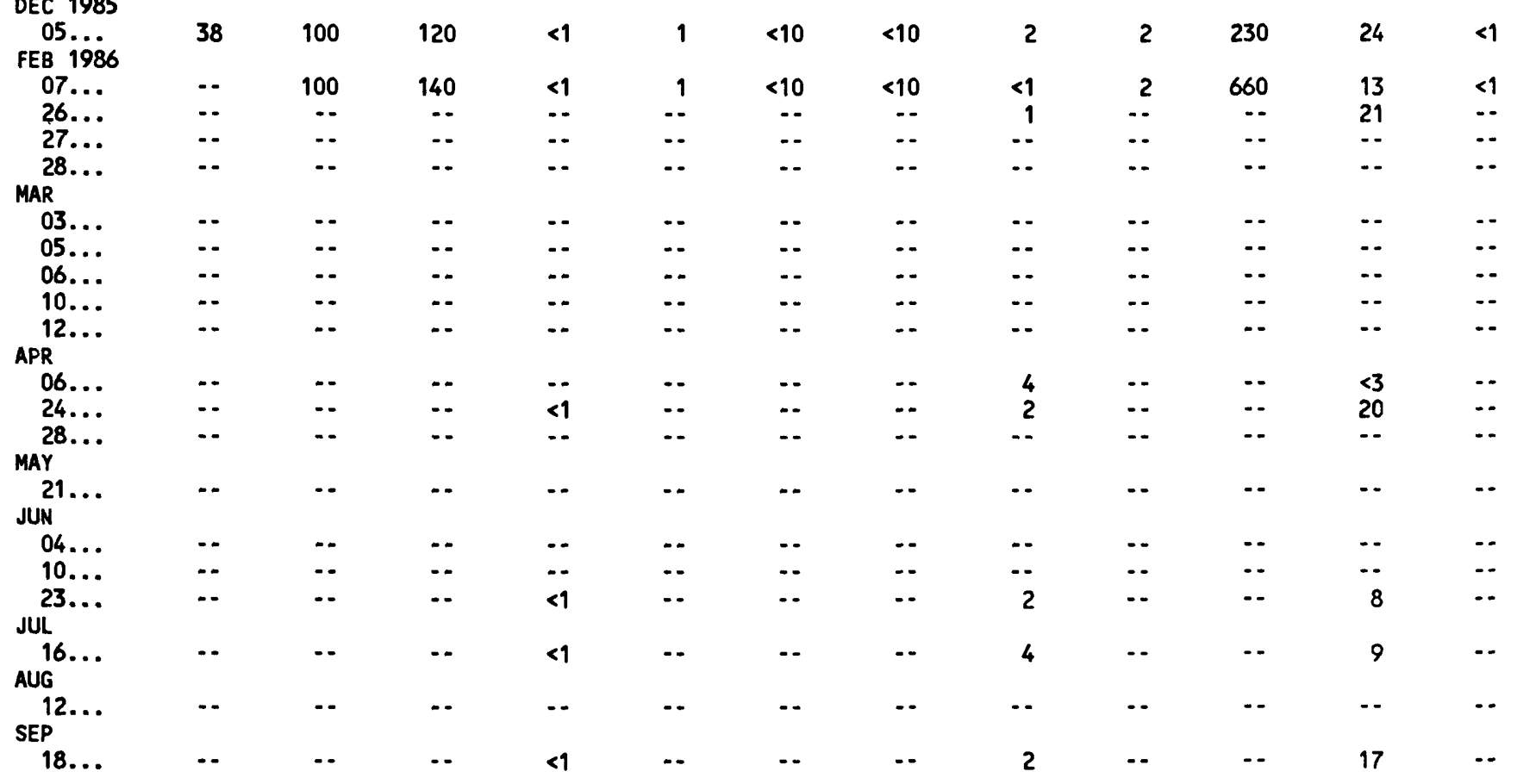


Table 35.--Chemical analyses of surface-water samples collected at Whi tewood Creek above Vale, S. Dak. - - Cont inued

\section{WATER QUALITY DATA}

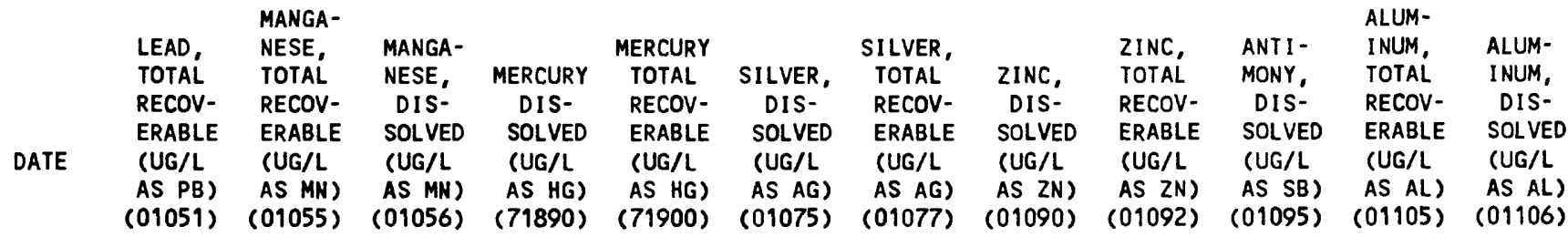

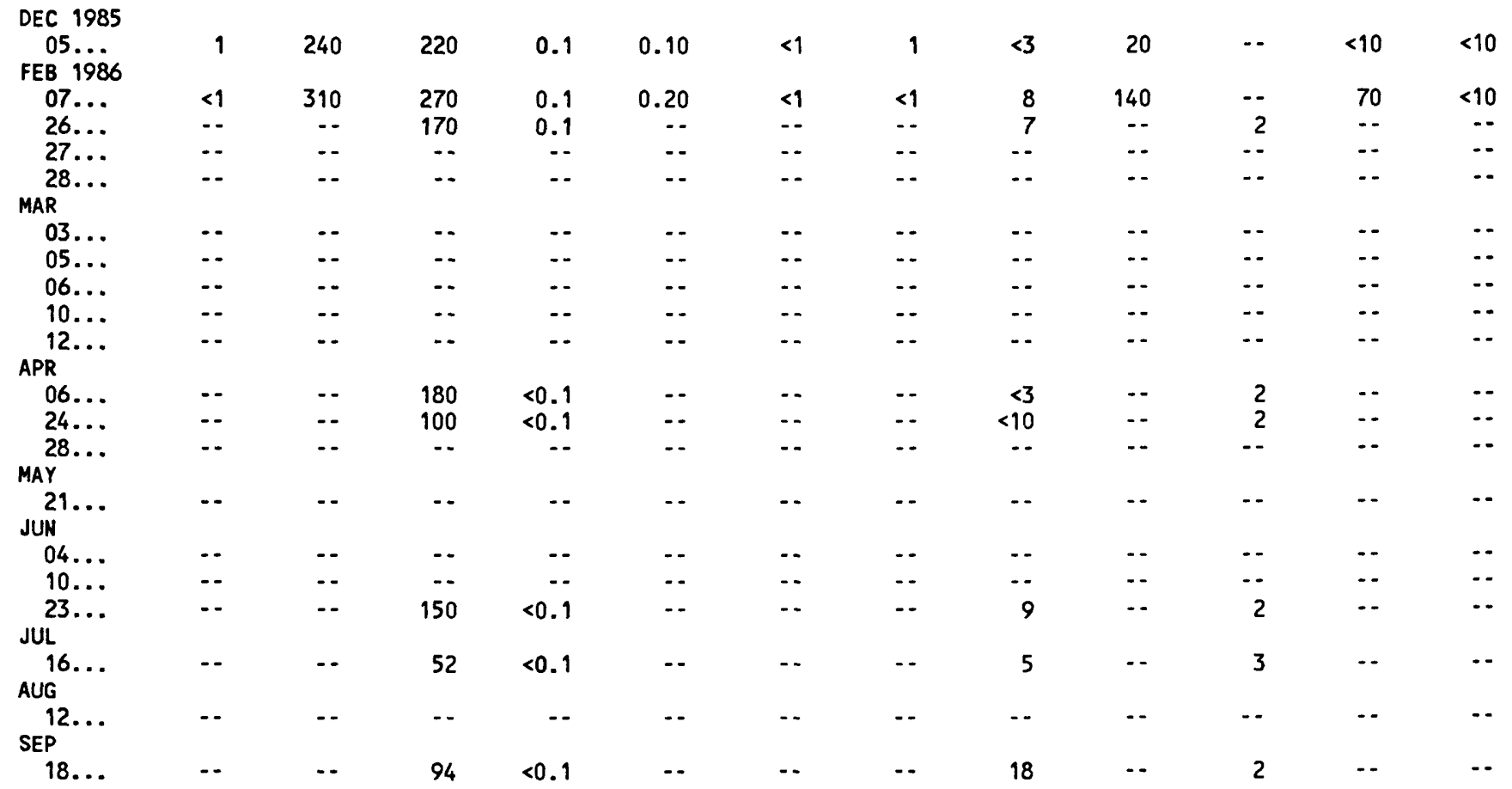


Table 35.--Chemical analyses of surface-water samples collected at

Whitewood Creek above Vale, S. Dak.--Cont inued

WATER QUALITY DATA

\begin{tabular}{|c|c|c|c|c|c|c|c|c|c|c|}
\hline $\begin{array}{l}\text { SELE- } \\
\text { NIUM, } \\
\text { DIS- } \\
\text { SOLVED } \\
\text { (UG/L } \\
\text { AS SE) } \\
01145 \text { ) }\end{array}$ & $\begin{array}{l}\text { SELE- } \\
\text { NIUM, } \\
\text { TOTAL } \\
\text { (UG/L } \\
\text { AS SE) } \\
\text { (01147) }\end{array}$ & $\begin{array}{l}\text { SEDI - } \\
\text { MENT, } \\
\text { SUS- } \\
\text { PENDED } \\
\text { (MG/L) } \\
(80154)\end{array}$ & $\begin{array}{l}\text { SED. } \\
\text { SUSP. } \\
\text { SIEVE } \\
\text { DIAM. } \\
\text { \% FINER } \\
\text { THAN } \\
.062 \text { MM } \\
(70331)\end{array}$ & $\begin{array}{l}\text { SED. } \\
\text { SUSP. } \\
\text { FALL } \\
\text { DIAM. } \\
\times \text { FINER } \\
\text { THAN } \\
.002 \text { MM } \\
(70337)\end{array}$ & $\begin{array}{l}\text { SED. } \\
\text { SUSP. } \\
\text { FALL } \\
\text { DIAM. } \\
\text { \% FINER } \\
\text { THAN } \\
.004 \text { MM } \\
\text { (70338) }\end{array}$ & $\begin{array}{l}\text { SED. } \\
\text { SUSP. } \\
\text { FALL } \\
\text { DIAM. } \\
\times \text { FINER } \\
\text { THAN } \\
.008 \text { MM } \\
\text { (70339) }\end{array}$ & $\begin{array}{l}\text { SED. } \\
\text { SUSP. } \\
\text { FALL } \\
\text { DIAM. } \\
\times \text { FINER } \\
\text { THAN } \\
.016 \mathrm{MM} \\
(70340)\end{array}$ & $\begin{array}{l}\text { SED. } \\
\text { SUSP. } \\
\text { FALL } \\
\text { DIAM. } \\
\times \text { FINER } \\
\text { THAN } \\
.062 \text { MM } \\
\text { (70342) }\end{array}$ & $\begin{array}{l}\text { SED. } \\
\text { SUSP. } \\
\text { FALL } \\
\text { DIAM. } \\
\times \text { FINER } \\
\text { THAN } \\
.125 \mathrm{MM} \\
(70343)\end{array}$ & $\begin{array}{l}\text { SED. } \\
\text { SUSP. } \\
\text { FALL } \\
\text { DIAM. } \\
\text { \% FINER } \\
\text { THAN } \\
.250 \text { MM } \\
\text { (70344) }\end{array}$ \\
\hline
\end{tabular}

DEC 1985

$05 \ldots$

FEB 1986

$07 . .$.

26...

$27 . .$.

28...

MAR

03...

$05 . .$.

$06 . .$.

$10 . .$.

12

APR

$06 . .$.

$24 \ldots$

28...

MAY

$21 .$.

JUN

$04 \ldots$

$10 . .$.

23...

JUL

$16 . .$.

AUG

$12 . .$.

$\begin{array}{rrrr}2 & 2 & 40 & 45 \\ 3 & 3 & 17 & 70 \\ 2 & -- & 1160 & - \\ - & -- & 167 & 97 \\ - & -- & 46 & - \\ & & & \\ - & -- & 44 & - \\ - & -- & 37 & - \\ - & -- & 37 & - \\ - & -- & 42 & - \\ & -- & 20 & - \\ -- & -. & 123 & 98 \\ - & -- & 380 & - \\ -- & 193 & 96\end{array}$

45

70

97

38

$46 \quad 55$

55

69

$-$

-.

$-2$

$-\cdot$

-.

$-$

$\cdots$

-

$98 \quad \ldots$

96

34

43

-- 13

90

$\begin{array}{rrr}\ldots & \ldots & 86 \\ \ldots & \ldots & 1390 \\ \ldots & \ldots & 20\end{array}$

$\begin{array}{rrr}-- & - & 86 \\ -- & \ldots & 1390 \\ -- & -. & 20\end{array}$

--

--

39

SEP

$18 . .$.

-. 7

95

98

82 
Table 36. - Chemical analyses of surface-water samples collected at Belle Fourche River near Sturgis, S. Dak. WATER QUALITY DATA

\begin{tabular}{|c|c|c|c|c|c|c|c|c|c|c|c|}
\hline DATE & TIME & $\begin{array}{l}\text { TEMPER- } \\
\text { ATURE } \\
\text { WATER } \\
\text { (DEG C) } \\
(00010)\end{array}$ & $\begin{array}{l}\text { BARO- } \\
\text { METRIC } \\
\text { PRES- } \\
\text { SURE } \\
\text { (MM } \\
\text { OF } \\
\text { HG) } \\
(00025)\end{array}$ & $\begin{array}{l}\text { STREAM- } \\
\text { FLOW, } \\
\text { INSTAN- } \\
\text { TANEOUS } \\
\text { (CFS) } \\
\text { (00061) }\end{array}$ & $\begin{array}{l}\text { SPE- } \\
\text { CIFIC } \\
\text { CON- } \\
\text { DUCT- } \\
\text { ANCE } \\
\text { (US/CM) } \\
(00095)\end{array}$ & $\begin{array}{c}\text { SPE- } \\
\text { CIFIC } \\
\text { CON- } \\
\text { DUCT- } \\
\text { ANCE } \\
\text { LAB } \\
\text { (US/CM) } \\
(90095)\end{array}$ & $\begin{array}{c}\text { OXYGEN, } \\
\text { DIS- } \\
\text { SOLVED } \\
(M G / L) \\
(00300)\end{array}$ & $\begin{array}{l}\text { OXYGEN, } \\
\text { DIS- } \\
\text { SOLVED } \\
\text { (PER- } \\
\text { CENT } \\
\text { SATUR- } \\
\text { ATION) } \\
\text { (O0301) }\end{array}$ & $\begin{array}{c}\text { PH } \\
\text { (STAND- } \\
\text { ARD } \\
\text { UNITS) } \\
(00400)\end{array}$ & $\begin{array}{c}\text { PH } \\
\text { LAB } \\
\text { (STAND- } \\
\text { ARD } \\
\text { UNITS) } \\
\text { (00403) }\end{array}$ & $\begin{array}{c}\text { NITRO- } \\
\text { GEN, } \\
\text { NO2+NO3 } \\
\text { DIS- } \\
\text { SOLVED } \\
\text { (MG/L } \\
\text { AS N) } \\
(00631)\end{array}$ \\
\hline
\end{tabular}

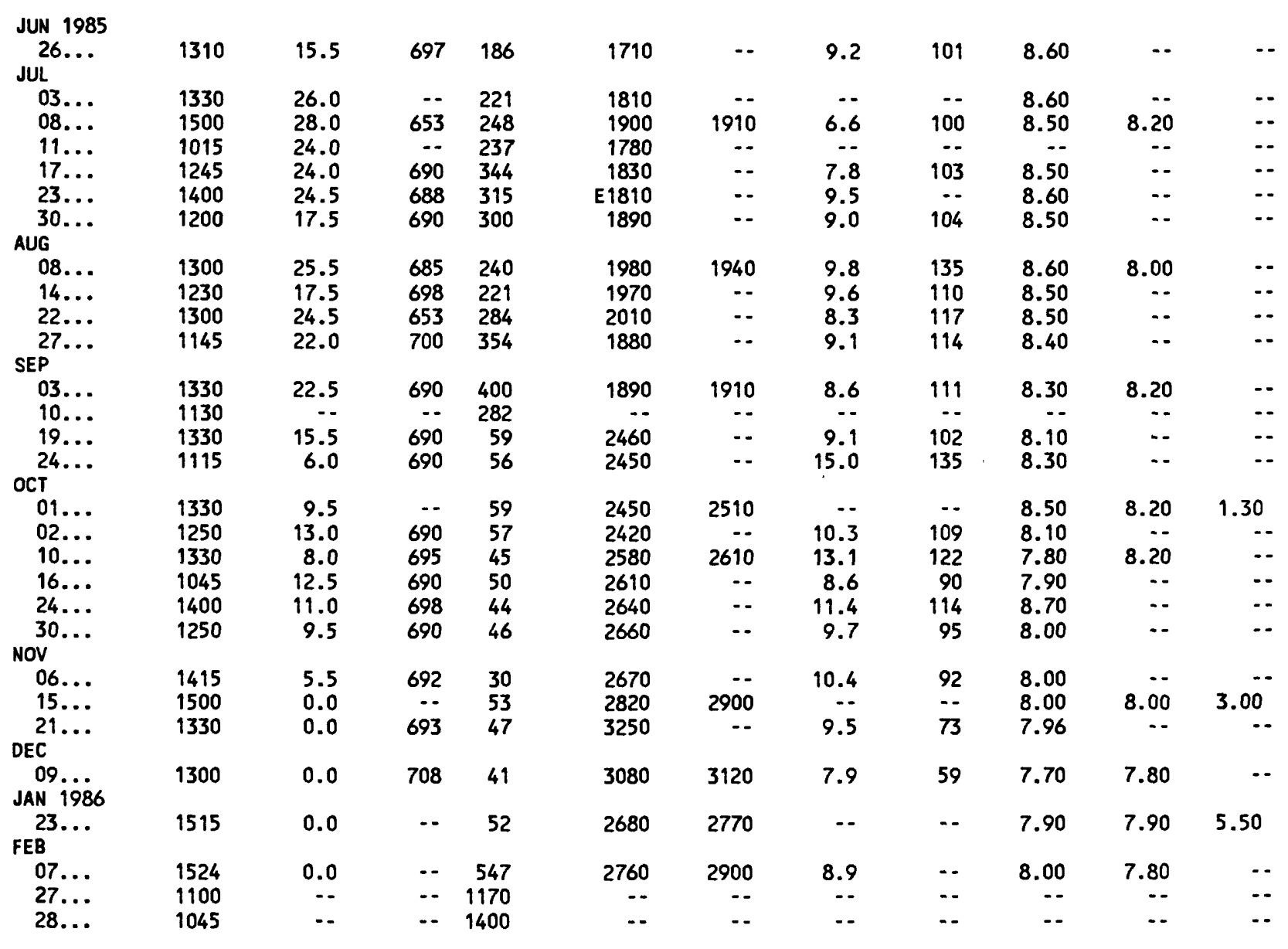


Table 36. --Chemical analyses of surface-water samples collected at

Belle Fourche River near Sturgis, S. Dak.--Cont inued

WATER QUALITY DATA

\begin{tabular}{|c|c|c|c|c|c|c|c|c|c|c|}
\hline $\begin{array}{l}\text { PHOS- } \\
\text { PHORUS, } \\
\text { TOTAL } \\
\text { (MG/L } \\
\text { AS P) } \\
(00665)\end{array}$ & $\begin{array}{c}\text { PHOS- } \\
\text { PHORUS, } \\
\text { DIS- } \\
\text { SOLVED } \\
\text { (MG/L } \\
\text { AS P) } \\
(00666)\end{array}$ & $\begin{array}{l}\text { CARBON, } \\
\text { ORGANIC } \\
\text { TOTAL } \\
\text { (MG/L } \\
\text { AS C) } \\
(00680)\end{array}$ & $\begin{array}{l}\text { CARBON, } \\
\text { ORGANIC } \\
\text { DIS- } \\
\text { SOLVED } \\
\text { (MG/L } \\
\text { AS C) } \\
(00681)\end{array}$ & $\begin{array}{c}\text { CYANIDE } \\
\text { TOTAL } \\
\text { (MG/L } \\
\text { AS CN) } \\
(00720)\end{array}$ & $\begin{array}{l}\text { CYANIDE } \\
\text { DIS- } \\
\text { SOLVED } \\
\text { (MG/L } \\
\text { AS CN) } \\
(00723)\end{array}$ & $\begin{array}{l}\text { SOLIDS, } \\
\text { RESIDUE } \\
\text { AT } 180 \\
\text { DEG. C } \\
\text { DIS- } \\
\text { SOLVED } \\
(M G / L) \\
(70300)\end{array}$ & $\begin{array}{l}\text { SOLIDS, } \\
\text { SUM OF } \\
\text { CONSTI- } \\
\text { TUENTS, } \\
\text { DIS- } \\
\text { SOLVED } \\
\text { (MG/L) } \\
(70301)\end{array}$ & $\begin{array}{l}\text { HARD- } \\
\text { NESS } \\
\text { (MG/L } \\
\text { AS } \\
\text { CACO3) } \\
(00900)\end{array}$ & $\begin{array}{l}\text { HARD- } \\
\text { NESS } \\
\text { NONCAR- } \\
\text { BONATE } \\
\text { (MG/L } \\
\text { AS } \\
\text { CACO3) } \\
(95902)\end{array}$ & $\begin{array}{c}\text { ALKA- } \\
\text { LINITY } \\
\text { LAB } \\
\text { (MG/L } \\
\text { AS } \\
\text { CACO3) } \\
(90410)\end{array}$ \\
\hline
\end{tabular}

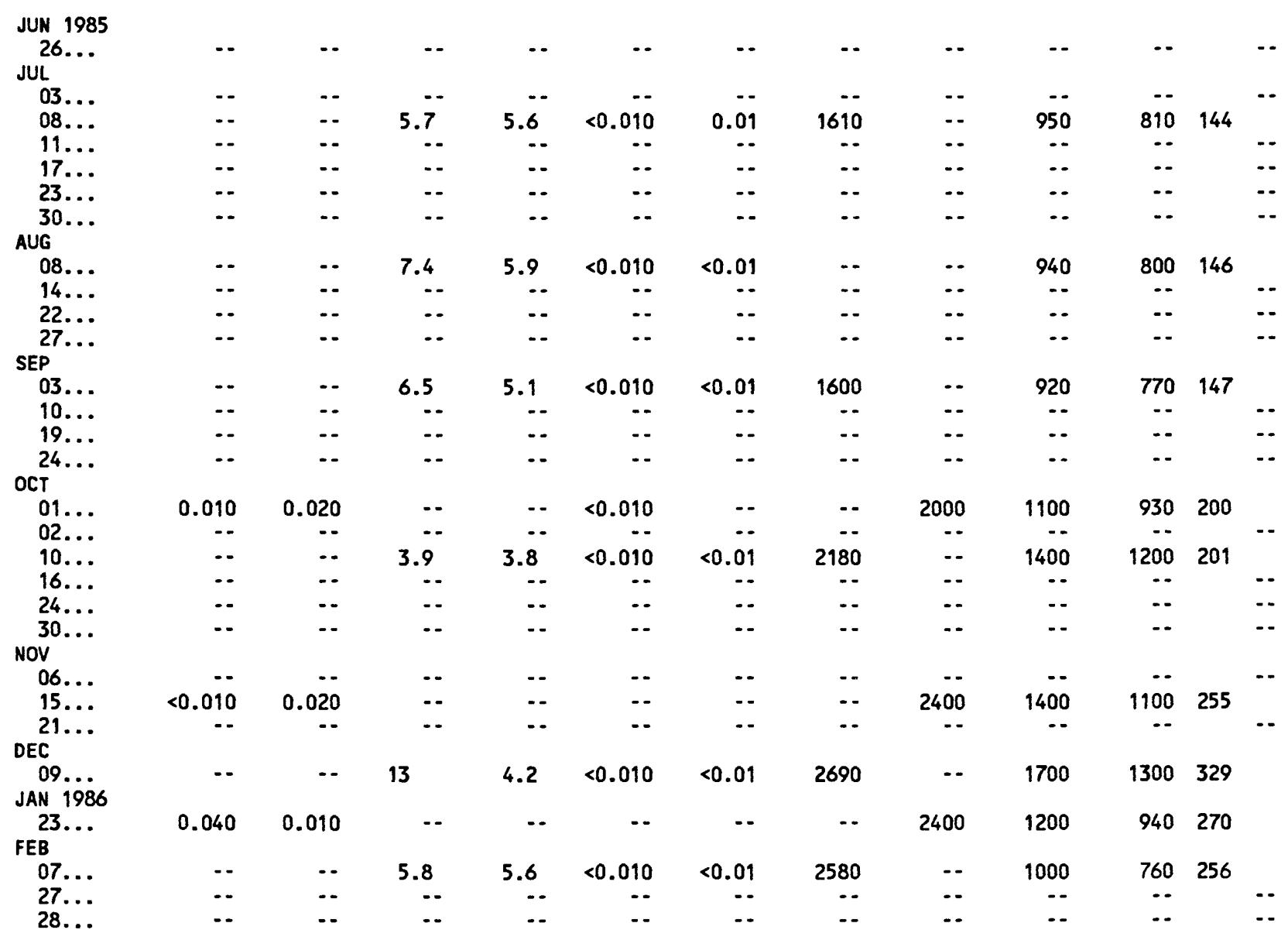


Table 36.--Chemical analyses of surface-water samples collected at Bel le Fourche River near Sturgis, S. Dak. --Cont inued

WATER QUALITY DATA

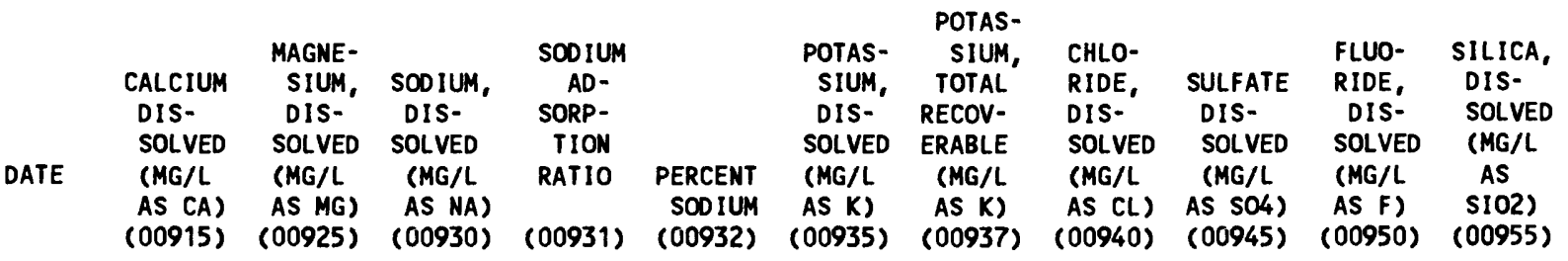

\begin{tabular}{|c|c|c|c|c|c|c|c|c|c|c|c|c|c|c|}
\hline $\begin{array}{c}\text { JUN } 1985 \\
26 \ldots \\
\text { JUL }\end{array}$ & & $\cdots$ & & $\cdots$ & & -- & $\cdots$ & -- & -- & $\cdots$ & $-\cdot$ & $-\cdot$ & -- & $\cdots$ \\
\hline $03 \ldots$ & & -- & & -- & & $-\cdot$ & $\cdots$ & -- & -. & -. & -. & -. & $\cdots$ & -- \\
\hline $08 \ldots$ & 230 & & 91 & & 120 & & 2 & 21 & 8.5 & 9.1 & 5.0 & 920 & -. & -- \\
\hline $11 \ldots$ & & -- & & $\cdots$ & & -- & - & -- & -. & - & -. & -. & $\cdots$ & $\cdots$ \\
\hline $17 \ldots$ & & -- & & $\cdots$ & & -- & - & -- & - & -- & -- & -. & -- & $\cdots$ \\
\hline $23 .$. & & -- & & $-\cdot$ & & -- & -- & $\cdots$ & -- & $\cdots$ & -- & $-\cdot$ & $\cdots$ & -- \\
\hline $30 \ldots$ & & -- & & $\cdots$ & & -- & -- & $\cdots$ & $\cdots$ & $\cdots$ & $\cdots$ & $\cdots$ & $\cdots$ & $\cdots$ \\
\hline AUG & & & & & & & & & & & & & & \\
\hline $08 \ldots$ & 230 & & 90 & & 120 & & 2 & 21 & 8.8 & 8.2 & $\cdots$ & $\cdots$ & $\cdots$ & $\cdots$ \\
\hline $14 \ldots$ & & -- & & -- & & $\cdots$ & - & -- & -. & - & $\cdot-$ & $\cdots$ & $-\cdot$ & $-\cdot$ \\
\hline $22 \ldots$ & & -- & & -- & & -- & -- & -- & -- & - & $\cdots$ & -- & $-\cdot$ & $\cdots$ \\
\hline$\underset{\text { SEP }}{27 \ldots}$ & & $-\cdot$ & & $\cdots$ & & -- & $\cdots$ & $\cdots$ & -- & -- & $\cdots$ & $\cdots$ & $-\cdot$ & $\cdots$ \\
\hline $03 .$. & 230 & & 83 & & 99 & & 1 & 19 & 8.9 & 81 & 16 & 070 & $\ldots$ & $\cdots$ \\
\hline $10 .$. & & $\cdots$ & & $\cdots$ & & $\cdots$ & .. & $\ldots$ & .. & 0.4 & .. & .. & $\ldots$ & -. \\
\hline $19 .$. & & - & & -- & & -- & -- & -. & -. & $\cdots$ & $\cdots$ & $\cdots$ & -- & - \\
\hline $\mathrm{OCT}^{24 \ldots}$ & & -- & & -- & & $-\cdot$ & -- & $\cdots$ & - & $\cdots$ & $\cdots$ & $\cdots$ & -- & $\cdots$ \\
\hline $01 \ldots$ & 270 & & 110 & & 160 & & 2 & 23 & 9.4 & -- & 15 & 1300 & 0.50 & 5.0 \\
\hline $02 \ldots$ & & $\cdots$ & & $\cdots$ & & $\cdots$ & $\cdots$ & $\ddot{-}$ & -- & -. & -- & $=$ & -- & $\cdots$ \\
\hline $10 \ldots$ & 320 & & 150 & & 180 & & 2 & 22 & 9.7 & 10 & 28 & 1400 & $-\cdot$ & $\cdots$ \\
\hline $16 \ldots$ & & -- & & -- & & -- & $\cdots$ & $-\cdot$ & -. & -. & -- & -- & -- & $\cdots$ \\
\hline $\begin{array}{l}24 \ldots \\
30 \ldots\end{array}$ & & -- & & $\cdots$ & & $\cdots$ & $\cdots$ & $\cdots$ & $\cdots$ & $\cdots$ & $\cdots$ & $\cdots$ & $\cdots$ & $\cdots$ \\
\hline $\begin{array}{l}30 . . \\
\text { Nov }\end{array}$ & & $\cdots$ & & $-\cdot$ & & -- & -- & -- & $\cdots$ & $\cdots$ & $\cdots$ & $\cdots$ & $\cdots$ & $\cdots$ \\
\hline $06 .$. & & $\cdots$ & & $\cdots$ & & -- & -. & -. & $\cdots$ & $\cdots$ & -- & $-\cdot$ & -- & .. \\
\hline $15 \ldots$ & 290 & & 160 & & 220 & & 3 & 26 & 10 & $\cdots$ & 29 & 1500 & 0.50 & 5.7 \\
\hline${ }_{D E C}^{21 \ldots}$ & & $\cdots$ & & -- & & -- & -- & $-\cdot$ & - & $\cdots$ & -- & -- & $\cdots$ & - \\
\hline JAN 19986 & 350 & & 190 & & 250 & & 3 & 25 & 10 & 15 & 39 & 1700 & -- & $\cdots$ \\
\hline$\underset{\text { FEB }}{23 \ldots}$ & 270 & & 130 & & 230 & & 3 & 29 & 10 & $\cdots$ & 51 & 1500 & 0.50 & 6.2 \\
\hline $07 \ldots$ & 210 & & 120 & & 270 & & 4 & 36 & 11 & 10 & 31 & 1400 & $\cdots$ & $\cdots$ \\
\hline $\begin{array}{l}27 \ldots \\
28 \ldots\end{array}$ & & $\begin{array}{l}-- \\
-\end{array}$ & & $\because$ & & $\cdots$ & -- & $\cdots$ & -- & $\cdots$ & - & - & $-\cdot$ & $\cdots$ \\
\hline & & 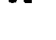 & & 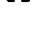 & & 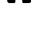 & $\cdots$ & $\cdots$ & $\cdots$ & $\cdots$ & $\cdots$ & $\cdots$ & $\cdots$ & $\cdots$ \\
\hline
\end{tabular}


Table 36.--Chemical analyses of surface-water samples collected at Belle Fourche River near Sturgis, S. Dak.--Continued

WATER QUALITY DATA

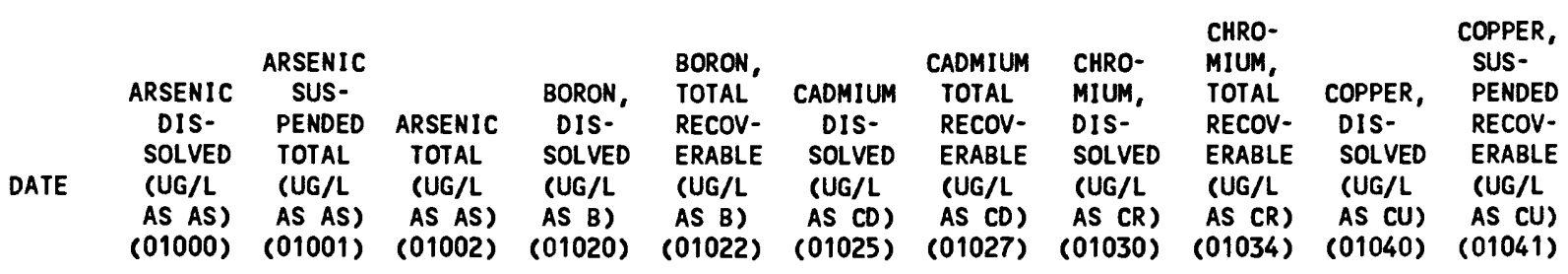

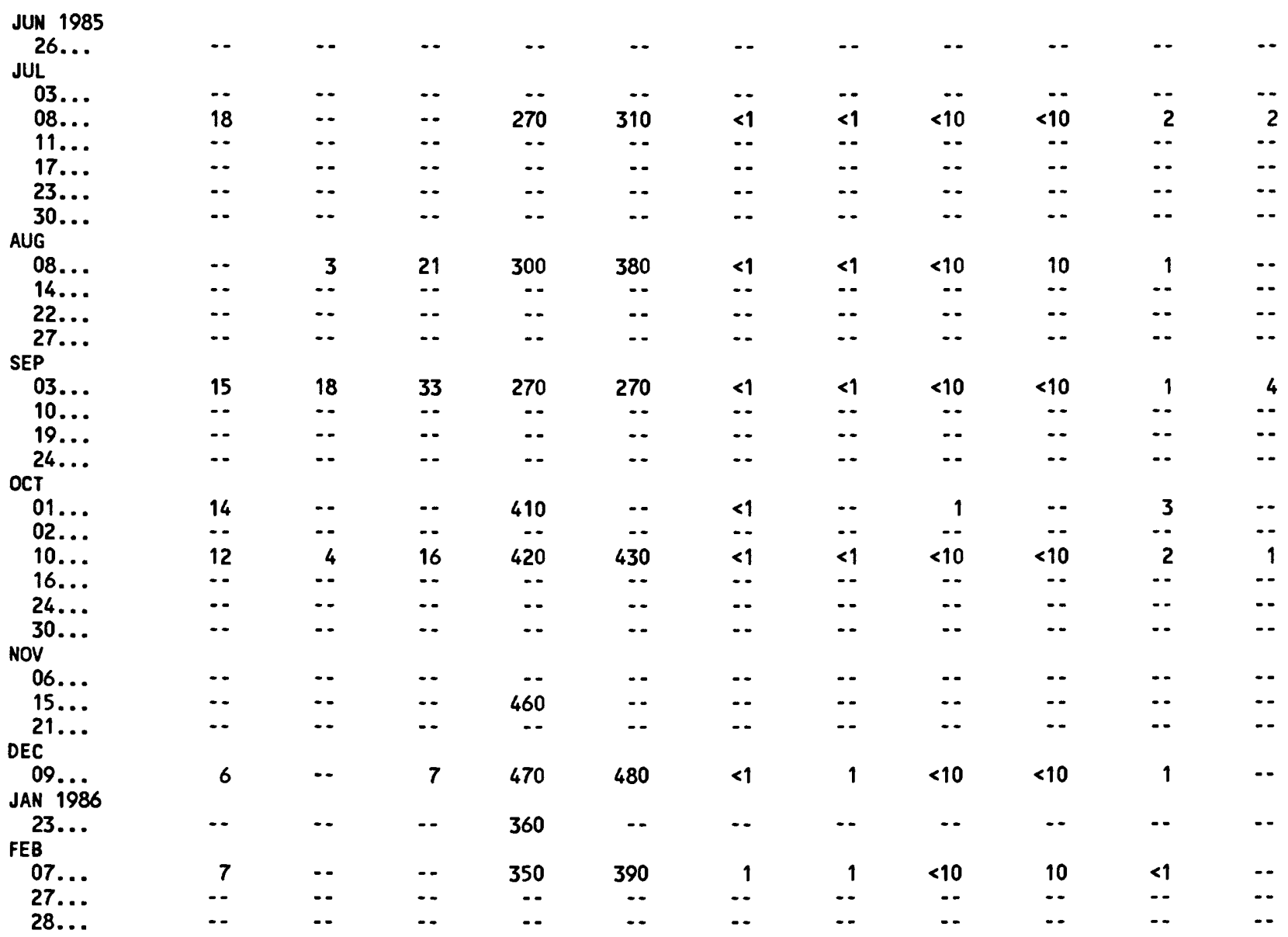


Table 36.-- Chemical analyses of surface-water samples collected at

Belle Fourche River near Sturgis, S. Dak.--Cont inued

WATER QUALITY DATA

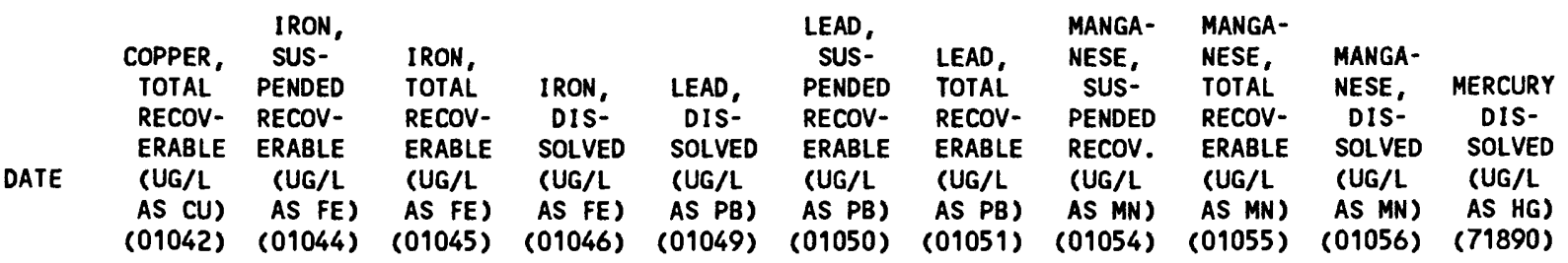

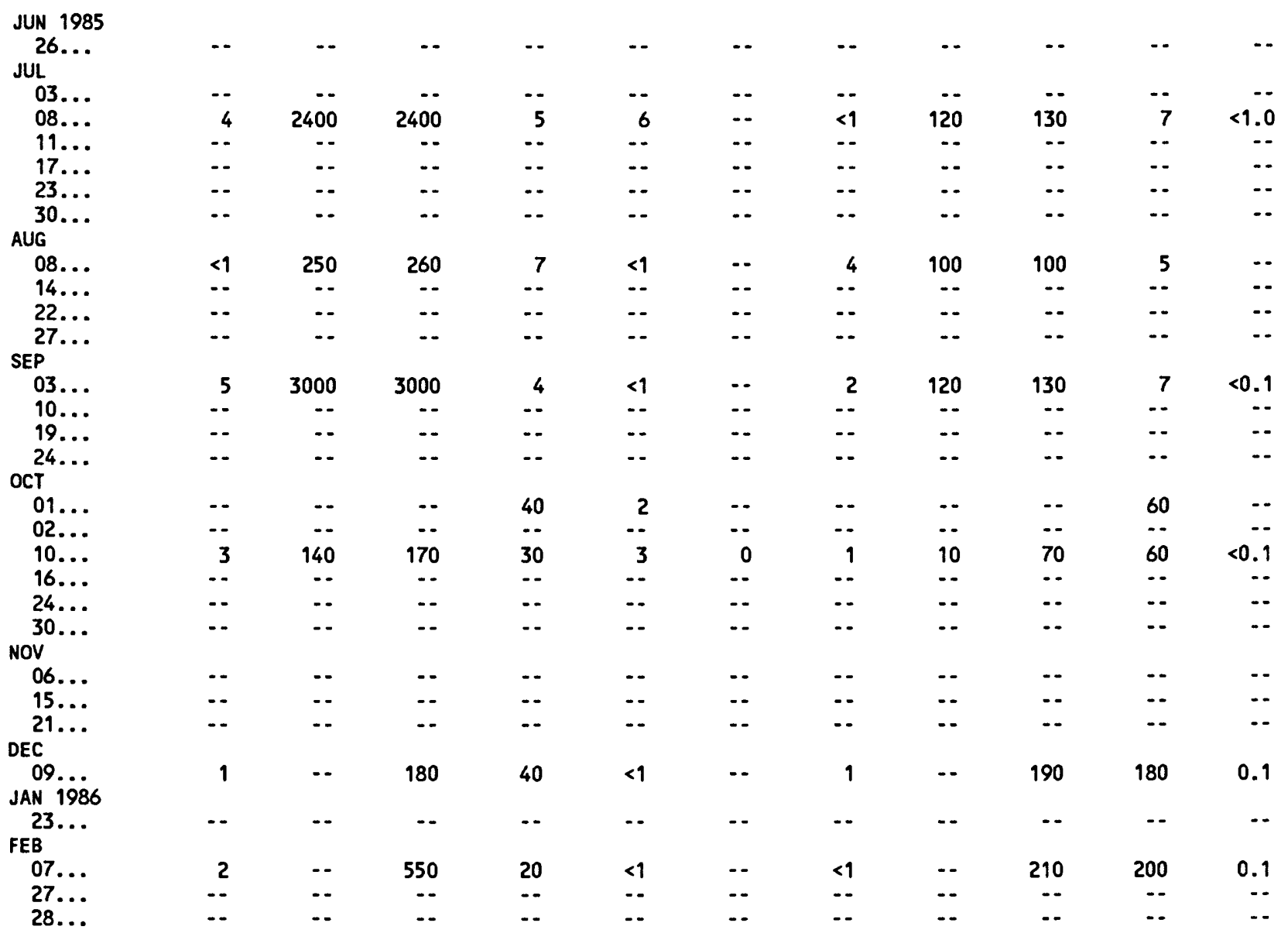


Table 36.--Chemical analyses of surface-water samples collected at Belle Fourche River near Sturgis, S. Dak.--Cont inued

WATER QUALITY DATA

\begin{tabular}{|c|c|c|c|c|c|c|c|c|c|c|c|}
\hline & $\begin{array}{l}\text { MERCURY } \\
\text { TOTAL } \\
\text { RECOV- } \\
\text { ERABLE }\end{array}$ & $\begin{array}{c}\text { SILVER, } \\
\text { DIS- } \\
\text { SOLVED }\end{array}$ & $\begin{array}{l}\text { SILVER, } \\
\text { TOTAL } \\
\text { RECOV- } \\
\text { ERABLE }\end{array}$ & $\begin{array}{l}\text { ZINC, } \\
\text { DIS- } \\
\text { SOLVED }\end{array}$ & $\begin{array}{l}\text { ZINC, } \\
\text { SUS- } \\
\text { PENDED } \\
\text { RECOV- } \\
\text { ERABLE }\end{array}$ & $\begin{array}{l}\text { ZINC, } \\
\text { TOTAL } \\
\text { RECOV- } \\
\text { ERABLE }\end{array}$ & $\begin{array}{l}\text { ALUM- } \\
\text { INUM, } \\
\text { TOTAL } \\
\text { RECOV- } \\
\text { ERABLE }\end{array}$ & $\begin{array}{l}\text { ALUM- } \\
\text { INUM, } \\
\text { DIS- } \\
\text { SOLVED }\end{array}$ & $\begin{array}{l}\text { ALUM- } \\
\text { INUM, } \\
\text { SUS- } \\
\text { PENDED } \\
\text { RECOV. }\end{array}$ & $\begin{array}{l}\text { SELE- } \\
\text { NIUM, } \\
\text { DIS- } \\
\text { SOLVED }\end{array}$ & $\begin{array}{l}\text { SELE- } \\
\text { NIUM, } \\
\text { SUS- } \\
\text { PENDED } \\
\text { TOTAL }\end{array}$ \\
\hline & $\begin{array}{c}\text { (UG/L } \\
\text { AS HG) } \\
(71900)\end{array}$ & $\begin{array}{c}\text { (UG/L } \\
\text { AS AG) } \\
(01075)\end{array}$ & $\begin{array}{c}\text { (UG/L } \\
\text { AS AG) } \\
\text { (01077) }\end{array}$ & $\begin{array}{c}\text { (UG/L } \\
\text { AS ZN) } \\
(01090)\end{array}$ & $\begin{array}{c}\text { (UG/L } \\
\text { AS ZN) } \\
(01091)\end{array}$ & $\begin{array}{c}\text { (UG/L } \\
\text { AS ZN) } \\
(01092)\end{array}$ & $\begin{array}{l}\text { UG/L } \\
\text { AS AL) } \\
(01105)\end{array}$ & $\begin{array}{l}(U G / L \\
\text { AS AL) } \\
(01106)\end{array}$ & $\begin{array}{l}\text { (UG/L } \\
\text { AS AL) } \\
(01107)\end{array}$ & $\begin{array}{l}\text { (UG/L } \\
\text { AS SE) } \\
(01145)\end{array}$ & $\begin{array}{l}\text { AS SE) } \\
(01146)\end{array}$ \\
\hline
\end{tabular}
JUN 1985

26 ...

JUL

03...

$08 . .$.

$11 \ldots$

$17 . .$.

$23 . .$.

$30 .$.

AUG

$08 . .$.

$14 \ldots$

$22 . .$.

$27 .$.

SEP

$03 . .$.

$10 .$. .

$19 . .$.

$24 \ldots$

OCT

$01 .$.

$02 . .$.

$10 . .$.

$16 . .$.

$24 . .$.

30...

NOV

06...

$15 . .$.

21...

DEC

$09 . .$.

JAN 1986

$23 .$. .

FEB

$07 . .$.

27...

$28 . .$.

$<0.10$

..

-.

$<0.10$

$<1$

-.

$-$

0.20

..

$-$

$<0.10$

$<0.10$

-.

$--$

$-\frac{100}{-1}$

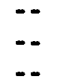

0.10

0.20

$<1$
--
--

$+-$

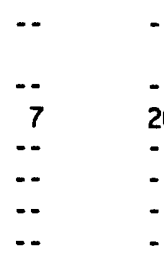$$
\begin{array}{ll}
7 & \\
-- & - \\
-- & -
\end{array}
$$

17
--
-
--

3
--
--

$\begin{array}{ll}- & 20 \\ - & -\end{array}$

$<1 \quad<10$

-. $\quad$.

-.

-

--

-.

$<10$

$\begin{array}{cc}1 & <10 \\ - & \end{array}$

$<1$
--

$--$

-.

10

--
3
--
--

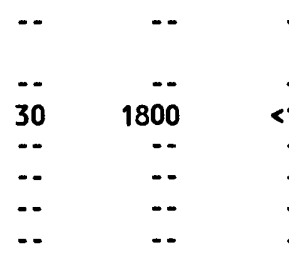

$10 \quad 790$

$790 \quad 10 \quad 780$

-.

-.

-.

$$
--
$$$$
-\cdot
$$

$\begin{array}{cr}20 & 1500 \\ \ldots & -- \\ -- & -. \\ -- & --\end{array}$

$10 \quad 1500$

-.

$-$

- $\quad$ -

$-$

$\begin{array}{ll}-- & -- \\ -- & --\end{array}$

$\begin{array}{ll}-- & -- \\ -- & 10\end{array}$

$-$

-.

40

$<10$

-.

$\cdots$

-.

-.

$--$

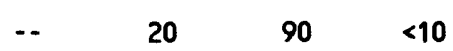

$<10$

$\cdots$

$\cdots \quad 12$

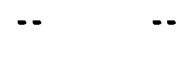


Table 36.--Chemical analyses of surface-water samples collected at

Belle Fourche River near Sturgis, S. Dak.--Cont inued

WATER QUALITY DATA

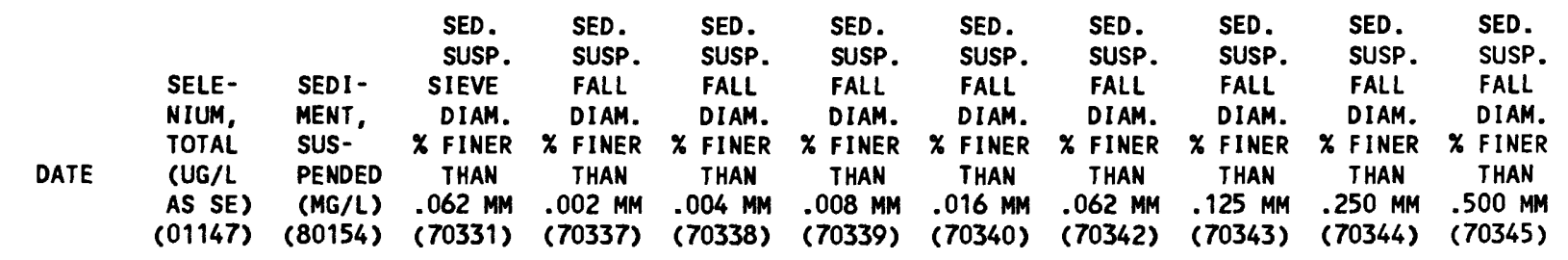

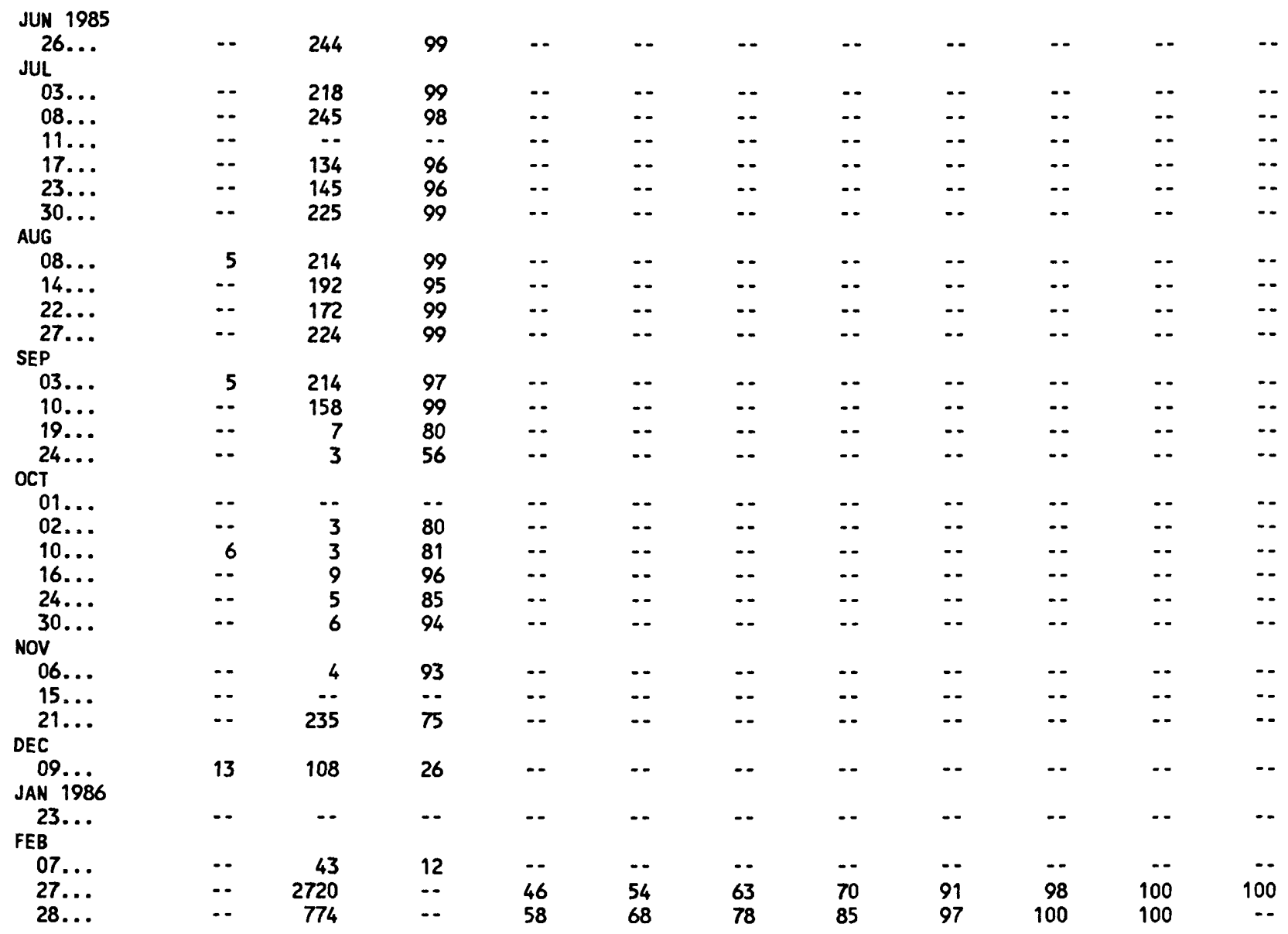


Table 36.--Chemical analyses of surface-water samples collected at

Belle Fourche River near Sturgis, S. Dak.--Cont inued

\section{WATER QUALITY DATA}

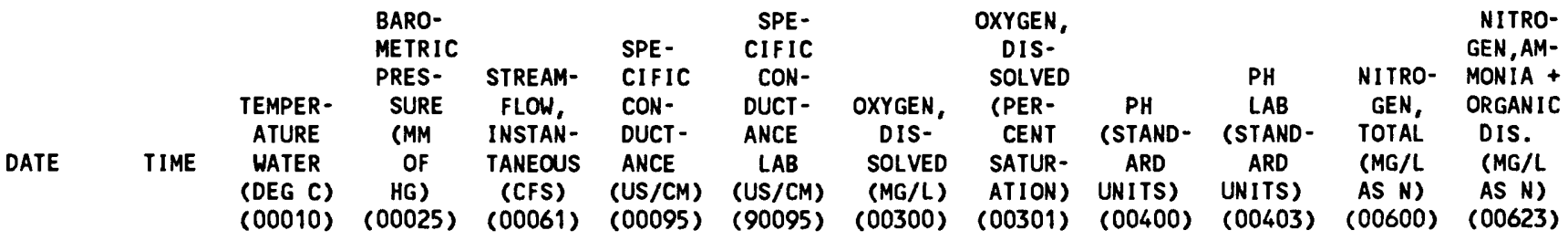

\begin{tabular}{|c|c|c|c|c|c|c|c|c|c|c|c|c|}
\hline MAR 1986 & & & & & & & & & & & & \\
\hline 03... & 1400 & 1.0 & 704 & 1190 & 1060 & 1140 & 10.9 & 83 & 7.90 & 7.70 & $\cdots$ & 1.5 \\
\hline $05 \ldots$ & 1015 & - & -. & 1440 & - & -. & -. & -- & -- & -- & $\cdots$ & -. \\
\hline $06 \ldots$ & 1400 & -- & -- & 1800 & $\cdots$ & -- & $\cdots$ & $\cdots$ & $\cdots$ & $\cdots$ & - & $-\cdot$ \\
\hline $10 \ldots$ & 1300 & $\cdots$ & - & 788 & $\cdots$ & -- & -- & $\cdots$ & $\cdots$ & - & -- & $-\cdot$ \\
\hline $12 \ldots$ & 1200 & - & - & 770 & -- & $\cdots$ & -- & -- & -- & -- & $\cdots$ & -. \\
\hline$\underset{A P R}{21 \cdots}$ & 1205 & 9.5 & 696 & 236 & 2160 & $\cdots$ & 10.5 & 101 & 8.30 & -- & - & -- \\
\hline$\underset{\text { MAY }}{09 . . .}$ & 1230 & 12.5 & 697 & 338 & 1520 & 1530 & 9.1 & 94 & 8.10 & 7.70 & $\cdots$ & 0.50 \\
\hline $\begin{array}{l}01 \ldots \\
08 \ldots\end{array}$ & $\begin{array}{l}1425 \\
1445\end{array}$ & $\begin{array}{r}13.5 \\
9.0\end{array}$ & $\begin{array}{c}702 \\
\ldots\end{array}$ & $\begin{array}{l}401 \\
230\end{array}$ & $\begin{array}{l}1460 \\
2110\end{array}$ & $\begin{array}{l}1480 \\
2130\end{array}$ & -- & $\cdots$ & 8.50 & $\begin{array}{l}7.90 \\
8.10\end{array}$ & $\cdots$ & $0 . \overline{-.}$ \\
\hline JUN & & & & & & & & & & & & \\
\hline JUL $02 \ldots$ & 1130 & 23.5 & 693 & 92 & 2460 & -- & 7.1 & 93 & 8.40 & 8.20 & -- & 0.40 \\
\hline $\begin{array}{l}08 \ldots \\
A \cup G\end{array}$ & 1430 & 28.0 & 698 & 252 & 1820 & 1910 & 8.2 & 116 & 8.30 & 8.10 & $\cdots$ & 0.50 \\
\hline${ }_{\text {SEP }}^{13 \ldots}$ & 1500 & 22.0 & 692 & 315 & 1700 & 1730 & 9.3 & 118 & 8.35 & 8.00 & 1.1 & 0.40 \\
\hline $\begin{array}{l}19 \ldots \\
25 \ldots \\
26 \ldots\end{array}$ & $\begin{array}{l}1415 \\
1254 \\
1315\end{array}$ & $\begin{array}{l}15.0 \\
15.0 \\
13.5\end{array}$ & $\begin{array}{l}696 \\
679 \\
690\end{array}$ & $\begin{array}{r}233 \\
6460 \\
5360\end{array}$ & $\begin{array}{r}1930 \\
725 \\
621\end{array}$ & $\begin{array}{r}1920 \\
711 \\
-.\end{array}$ & $\begin{array}{r}10.1 \\
3.2 \\
6.8\end{array}$ & $\begin{array}{r}111 \\
36 \\
73\end{array}$ & $\begin{array}{r}8.20 \\
\ldots \\
8.20\end{array}$ & $\begin{array}{c}8.10 \\
7.70 \\
.-\end{array}$ & $\begin{array}{c}1.6 \\
2.3 \\
-.\end{array}$ & $\begin{array}{c}0.70 \\
0.90 \\
.-\end{array}$ \\
\hline
\end{tabular}


Table 36.--Chemical analyses of surface-water samples collected at Belle Fourche River near Sturgis, S. Dak.--Cont inued

\begin{tabular}{|c|c|c|c|c|c|c|c|c|c|c|c|c|}
\hline & & & & & WATER & QUALITY & DATA & & & & & \\
\hline ITE & $\begin{array}{l}\text { NITRO- } \\
\text { GEN, } \\
\text { NO2+NO3 } \\
\text { TOTAL } \\
\text { (MG/L } \\
\text { AS N) } \\
(00630)\end{array}$ & $\begin{array}{c}\text { NITRO- } \\
\text { GEN, } \\
\text { NO2+NO3 } \\
\text { DIS- } \\
\text { SOLVED } \\
\text { (MG/L } \\
\text { AS N) } \\
(00631)\end{array}$ & $\begin{array}{l}\text { PHOS- } \\
\text { PHORUS, } \\
\text { TOTAL } \\
\text { (MG/L } \\
\text { AS P) } \\
(00665)\end{array}$ & $\begin{array}{c}\text { PHOS- } \\
\text { PHORUS, } \\
\text { DIS- } \\
\text { SOLVED } \\
\text { (MG/L } \\
\text { AS P) } \\
(00666)\end{array}$ & $\begin{array}{c}\text { CARBON, } \\
\text { ORGANIC } \\
\text { TOTAL } \\
\text { (MG/L } \\
\text { AS C) } \\
(00680)\end{array}$ & $\begin{array}{l}\text { CARBON, } \\
\text { ORGANIC } \\
\text { DIS- } \\
\text { SOLVED } \\
(M G / L \\
\text { AS C) } \\
(00681)\end{array}$ & $\begin{array}{l}\text { CYANIDE } \\
\text { DIS- } \\
\text { SOLVED } \\
\text { (MG/L } \\
\text { AS CN) } \\
(00723)\end{array}$ & $\begin{array}{l}\text { SOLIDS, } \\
\text { RESIDUE } \\
\text { AT } 180 \\
\text { DEG. C } \\
\text { DIS- } \\
\text { SOLVED } \\
(M G / L) \\
(70300)\end{array}$ & $\begin{array}{l}\text { SOLIDS, } \\
\text { SUM OF } \\
\text { CONSTI - } \\
\text { TUENTS, } \\
\text { DIS- } \\
\text { SOLVED } \\
\text { (MG/L) } \\
(70301)\end{array}$ & $\begin{array}{l}\text { HARD- } \\
\text { NESS } \\
\text { (MG/L } \\
\text { AS } \\
\text { CACO3) } \\
(00900)\end{array}$ & $\begin{array}{l}\text { HARD- } \\
\text { NESS } \\
\text { NONCAR- } \\
\text { BONATE } \\
\text { (MG/L } \\
\text { AS } \\
\text { CACO3) } \\
(95902)\end{array}$ & $\begin{array}{l}\text { ALKA- } \\
\text { LINITY } \\
\text { LAB } \\
\text { (MG/L } \\
\text { AS } \\
\text { CACO3) } \\
(90410)\end{array}$ \\
\hline
\end{tabular}

\begin{tabular}{|c|c|c|c|c|c|c|c|c|c|c|c|c|}
\hline $\begin{array}{c}\text { MAR } 1986 \\
03 \ldots\end{array}$ & $\cdots$ & 3.40 & 0.540 & 0.030 & $\cdots$ & -- & $<0.01$ & 803 & 720 & 330 & 240 & 96 \\
\hline $05 \ldots$ & -- & - & - & - & $\cdots$ & -- & -. & -- & -. & -. & -- & \\
\hline $06 \ldots$ & $\cdots$ & $\cdots$ & $\cdots$ & $\cdots$ & $\cdots$ & $\cdots$ & -- & $\cdots$ & -- & -- & $\cdots$ & \\
\hline $10 \ldots$ & - & $\cdots$ & $\cdots$ & - & $\cdots$ & -- & -- & -- & - & -- & - & \\
\hline $12 \ldots$ & $\cdots$ & - & $\cdots$ & - & -- & -- & $\cdots$ & -- & $-\cdot$ & $\cdots$ & -- & \\
\hline${ }_{A P R}^{21} \cdots$ & $\cdots$ & $\cdots$ & - & -- & -- & - & - & -- & -- & -- & $\cdots$ & \\
\hline$\underset{\text { MAY }}{09 . . .}$ & $\cdots$ & 1.20 & 0.830 & 0.019 & 29 & 6.2 & $<0.01$ & 1160 & 1100 & 520 & 410 & 110 \\
\hline $\begin{array}{l}01 \ldots \\
08 \ldots\end{array}$ & $\begin{array}{r}1.00 \\
\ldots\end{array}$ & $1.40^{--}$ & $\begin{array}{l}0.230 \\
0.070\end{array}$ & $<0.005$ & $\begin{array}{l}17 \\
8.8\end{array}$ & $6 . \overline{7}$ & $<0 . \overline{01}$ & 1780 & 1600 & 840 & 660 & $\begin{array}{l}124 \\
181\end{array}$ \\
\hline $\begin{array}{l}\text { JUN } \\
02 \ldots\end{array}$ & $\cdots$ & 0.330 & 0.030 & $<0.005$ & 5.5 & 5.0 & $<0.01$ & 2180 & 2000 & 1100 & 970 & 163 \\
\hline $\begin{array}{l}\text { JUL } \\
\quad 08 \ldots\end{array}$ & $\therefore$ & 0.860 & 0.030 & 0.005 & 5.6 & 5.2 & $<0.01$ & 1640 & 1500 & 870 & 710 & 153 \\
\hline AUG & & & & & & & & & & & & \\
\hline SEP $^{13}$ & 0.300 & 0.260 & 0.050 & 0.007 & 4.6 & 4.4 & 0.01 & 1460 & 1300 & 790 & 650 & 143 \\
\hline $\begin{array}{l}19 . . . \\
25 \ldots\end{array}$ & $\begin{array}{l}1.10 \\
0.700\end{array}$ & $\begin{array}{l}1.10 \\
0.640\end{array}$ & $\begin{array}{l}0.020 \\
0.190\end{array}$ & $\begin{array}{l}0.005 \\
0.032\end{array}$ & $\begin{array}{l}4.5 \\
36\end{array}$ & $\begin{array}{l}3.6 \\
4.4\end{array}$ & $\begin{array}{r}0.01 \\
<0.01\end{array}$ & $\begin{array}{r}1620 \\
535\end{array}$ & $\begin{array}{r}1500 \\
500\end{array}$ & $\begin{array}{l}900 \\
260\end{array}$ & $\begin{array}{l}770 \\
150\end{array}$ & $\begin{array}{l}132 \\
107\end{array}$ \\
\hline 26 & -- & $\cdots$ & -- & $\cdots$ & 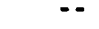 & -- & $\cdots$ & $\cdots$ & $\cdots$ & -- & -- & \\
\hline
\end{tabular}


Table 36.--Chemical analyses of surface-water samples collected at Belle Fourche River near Sturgis, S. Dak.--Cont inued

WATER QUALITY DATA

\begin{tabular}{|c|c|c|c|c|c|c|c|c|c|c|c|c|}
\hline ATE & $\begin{array}{l}\text { CALCIUM } \\
\text { DIS- } \\
\text { SOLVED } \\
\text { (MG/L } \\
\text { AS CA) } \\
(00915)\end{array}$ & $\begin{array}{l}\text { MAGNE- } \\
\text { SIUM, } \\
\text { DIS- } \\
\text { SOLVED } \\
\text { (MG/L } \\
\text { AS MG) } \\
(00925)\end{array}$ & $\begin{array}{l}\text { SODIUM, } \\
\text { DIS- } \\
\text { SOLVED } \\
\text { (MG/L } \\
\text { AS NA) } \\
(00930)\end{array}$ & $\begin{array}{c}\text { SODIUM } \\
\text { AD- } \\
\text { SORP- } \\
\text { TION } \\
\text { RATIO } \\
(00931)\end{array}$ & $\begin{array}{l}\text { PERCENT } \\
\text { SODIUM } \\
(00932)\end{array}$ & $\begin{array}{l}\text { POTAS- } \\
\text { SIUM, } \\
\text { DIS- } \\
\text { SOLVED } \\
\text { (MG/L } \\
\text { AS K) } \\
(00935)\end{array}$ & $\begin{array}{l}\text { CHLO- } \\
\text { RIDE, } \\
\text { DIS- } \\
\text { SOLVED } \\
(M G / L \\
\text { AS CL) } \\
(00940)\end{array}$ & $\begin{array}{l}\text { SULFATE } \\
\text { DIS- } \\
\text { SOLVED } \\
\text { (MG/L } \\
\text { AS SO4) } \\
(00945)\end{array}$ & $\begin{array}{l}\text { FLUO- } \\
\text { RIDE, } \\
\text { DIS- } \\
\text { SOLVED } \\
(M G / L \\
\text { AS F) } \\
(00950)\end{array}$ & $\begin{array}{l}\text { SILICA, } \\
\text { DIS- } \\
\text { SOLVED } \\
\text { (MG/L } \\
\text { AS } \\
\text { SIO2) } \\
(00955)\end{array}$ & $\begin{array}{l}\text { ARSENIC } \\
\text { DIS- } \\
\text { SOLVED } \\
\text { (UG/L } \\
\text { AS AS) } \\
(01000)\end{array}$ & $\begin{array}{l}\text { BORON, } \\
\text { DIS- } \\
\text { SOLVED } \\
\text { (UG/L } \\
\text { AS B) } \\
(01020)\end{array}$ \\
\hline
\end{tabular}

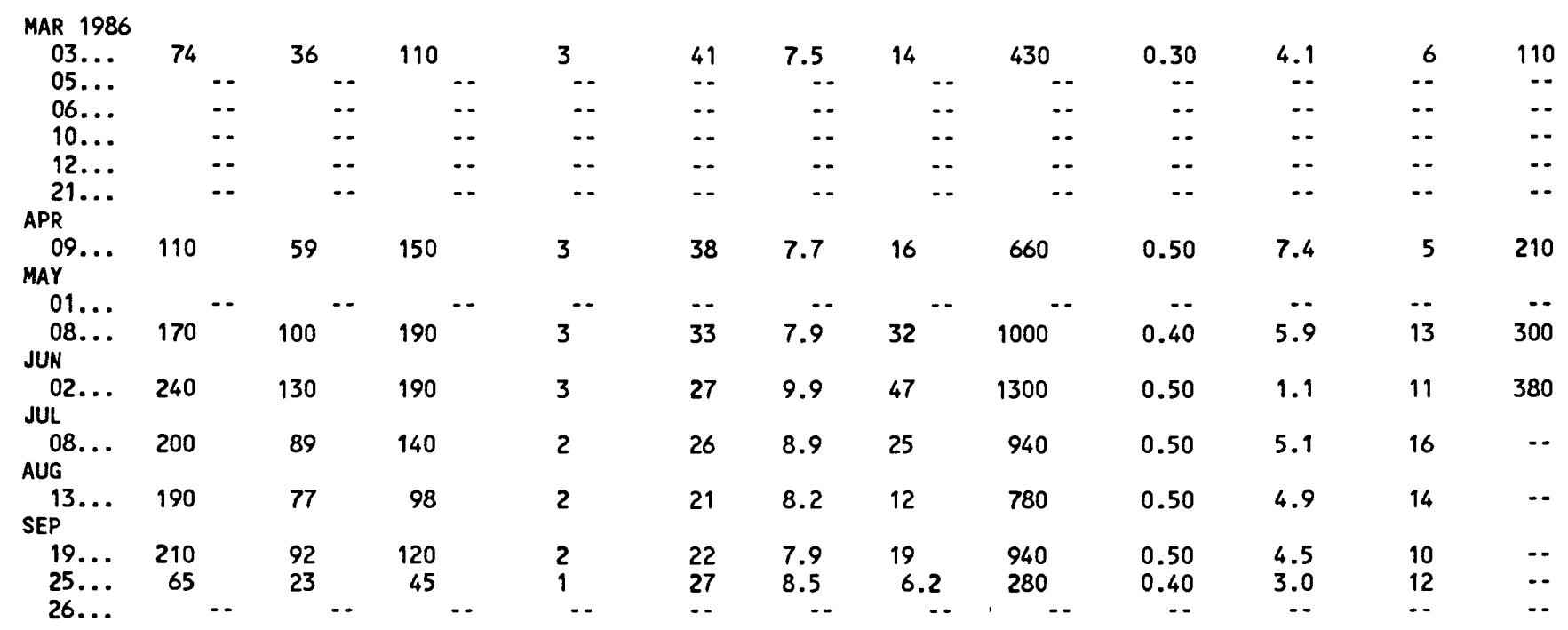


Table 36.--Chemical analyses of surface-water samples collected at Belle Fourche River near Sturgis, S. Dak. --Cont inued

WATER QUALITY DATA

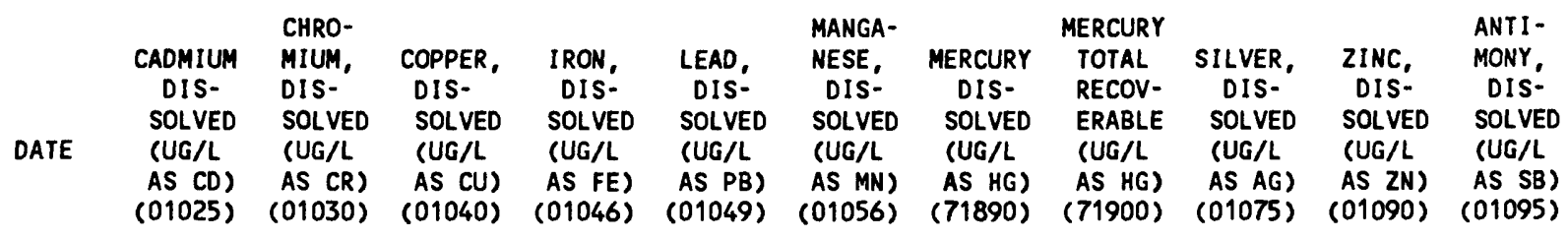

\begin{tabular}{|c|c|c|c|c|c|c|c|c|c|c|c|}
\hline \multicolumn{12}{|l|}{ MAR 1986} \\
\hline $03 . .$. & $<1$ & $<1$ & 1 & 19 & 1 & 56 & 0.1 & 1.1 & $<1$ & 6 & $<1$ \\
\hline $05 \ldots$ & -- & - & -- & -- & $\ldots$ & $\ldots$ & $\ldots$ & -. & - & -- & - \\
\hline $06 \ldots$ & $\ldots$ & $\ldots$ & $\ldots$ & .- & .- & $\ldots$ & $\ldots$ & $\ldots$ & $\ldots$ & -. & .- \\
\hline $10 \ldots$ & .. & .. & -- & -- & -. & -. & -. & -- & .. & -. & -. \\
\hline $12 \ldots$ & $\ldots$ & - - & -. & - & .. & $\ldots$ & $\ldots$ & $\ldots$ & $\cdots$ & -- & - \\
\hline $21 \ldots$ & $=-$ & -- & -- & .- & -- & $\ldots$ & -- & -- & -- & -- & $\cdots$ \\
\hline \multicolumn{12}{|l|}{ APR } \\
\hline $09 \ldots$ & $\cdots$ & $\cdots$ & 5 & 10 & -- & 2 & $<0.1$ & $\cdots$ & - & 5 & 1 \\
\hline \multicolumn{12}{|l|}{ MAY } \\
\hline $01 \ldots$ & -- & $\cdots$ & -. & -- & -- & $\cdots$ & -- & -- & -- & -- & $\cdots$ \\
\hline $08 \ldots$ & $<1$ & $\cdots$ & 4 & 20 & -- & 130 & $<0.1$ & $\cdots$ & $\cdots$ & $<10$ & $<1$ \\
\hline \multicolumn{12}{|l|}{ JUN } \\
\hline $02 \ldots$ & $<1$ & $\cdots$ & $<1$ & 40 & -- & 50 & $<0.1$ & $\cdots$ & $\cdots$ & $<10$ & $<1$ \\
\hline \multicolumn{12}{|l|}{ JUL } \\
\hline 08... & $<1$ & -- & 2 & 4 & $\cdots$ & 6 & $<0.1$ & $\cdots$ & $\cdots$ & 11 & $<1$ \\
\hline AUG & & & & & & & & & & & \\
\hline $\operatorname{SEP}^{13 \ldots}$ & $<1$ & $\cdots$ & 6 & 6 & $\cdots$ & 4 & $<0.1$ & $\cdots$ & $\cdots$ & 62 & 1 \\
\hline $19 .$. & $<1$ & -. & 1 & 3 & $\ldots$ & 17 & $<0.1$ & $\ldots$ & -- & 9 & $<1$ \\
\hline 25. & $<1$ & $\ldots$ & 1 & 8 & -- & 5 & $<0.1$ & $\ldots$ & -- & 11 & $<1$ \\
\hline $26 \ldots$ & -- & - & - & -- & - & $=$ & - & -- & -. & .. & - \\
\hline
\end{tabular}


Table 36.--Chemical analyses of surface-water samples collected at Belle Fourche River near Sturgis, S. Dak.--Cont inued

\begin{tabular}{|c|c|c|c|c|c|c|c|c|c|c|}
\hline \multicolumn{11}{|c|}{ WATER QUALITY DATA } \\
\hline $\begin{array}{l}\text { SELE- } \\
\text { NIUM, } \\
\text { DIS- } \\
\text { SOLVED } \\
\text { (UG/L } \\
\text { AS SE) } \\
(01145)\end{array}$ & $\begin{array}{l}\text { SEDI - } \\
\text { MENT, } \\
\text { SUS- } \\
\text { PENDED } \\
(M G / L) \\
(80154)\end{array}$ & $\begin{array}{c}\text { SED. } \\
\text { SUSP. } \\
\text { SIEVE } \\
\text { DIAM. } \\
\% \text { FINER } \\
\text { THAN } \\
.062 \mathrm{MM} \\
(70331)\end{array}$ & $\begin{array}{l}\text { SED. } \\
\text { SUSP. } \\
\text { FALL } \\
\text { DIAM. } \\
\% \text { FINER } \\
\text { THAN } \\
.002 \text { MM } \\
(70337)\end{array}$ & $\begin{array}{l}\text { SED. } \\
\text { SUSP. } \\
\text { FALL } \\
\text { DIAM. } \\
\% \text { FINER } \\
\text { THAN } \\
.004 \mathrm{MM} \\
(70338)\end{array}$ & $\begin{array}{l}\text { SED. } \\
\text { SUSP. } \\
\text { FALL } \\
\text { DIAM. } \\
\% \text { FINER } \\
\text { THAN } \\
.008 \mathrm{MM} \\
(70339)\end{array}$ & $\begin{array}{l}\text { SED. } \\
\text { SUSP. } \\
\text { FALL } \\
\text { DIAM. } \\
\% \text { FINER } \\
\text { THAN } \\
.016 \mathrm{MM} \\
(70340)\end{array}$ & $\begin{array}{l}\text { SED. } \\
\text { SUSP. } \\
\text { FALL } \\
\text { DIAM. } \\
\% \text { FINER } \\
\text { THAN } \\
.062 \mathrm{MM} \\
(70342)\end{array}$ & $\begin{array}{l}\text { SED. } \\
\text { SUSP. } \\
\text { FALL } \\
\text { DIAM. } \\
\% \text { FINER } \\
\text { THAN } \\
.125 \mathrm{MM} \\
(70343)\end{array}$ & $\begin{array}{l}\text { SED. } \\
\text { SUSP. } \\
\text { FALL } \\
\text { DIAM. } \\
\% \text { FINER } \\
\text { THAN } \\
.250 \text { MM } \\
(70344)\end{array}$ & $\begin{array}{l}\text { SED. } \\
\text { SUSP. } \\
\text { FALL } \\
\text { DIAM. } \\
\% \text { FINER } \\
\text { THAN } \\
.500 \text { MM } \\
(70345)\end{array}$ \\
\hline
\end{tabular}

MAR 1986

$03 . .$.

$05 .$.

$06 . .$.

$10 . .$.

$12 . .$.

$21 \ldots$ APR

$09 .$.

MAY

$01 .$.

103960

.. 4070

.. 4860

$\cdots \quad 1480$

$\therefore \quad 1320$

.- 339

$\begin{array}{lll}-- & 46 & 54 \\ -- & 57 & 67\end{array}$

54
67

64

76

--

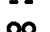

99
99

.. 2070

100

54

63

64

$08 .$.

JUN

$02 .$.

JUL

$08 . .$.

AUG

$13 . .$. SEP

$19 .$.

$25 .$.

$\begin{array}{lll}- & 870 \quad 100\end{array}$

74

74

83

83

$\begin{array}{ll}72 & 92 \\ 84 & 98 \\ 81 & 96 \\ 90 & 99 \\ 92 & -\end{array}$

$\begin{array}{rr}92 & 96 \\ 98 & 99 \\ 96 & 99 \\ 99 & 100 \\ -- & - \\ -- & -\end{array}$

$\begin{array}{rrr}96 & 99 & 100 \\ 99 & 100 & \ldots \\ 99 & 100 & -- \\ 100 & -- & -. \\ -- & -- & -. \\ -- & -. & -.\end{array}$

-. 66

$\begin{array}{ll}-. & 18\end{array}$

-. 54

-. 46

-. 21

-. $\quad 7070$

97

94

$71 \quad 82$

94

92

98

$96 \quad 100$

98

96

99

$4 \quad 50$

--
62

83

94

97

--

$99 \quad 100$ 
Table 37.-- Chemical analyses of surface-water samples collected at Cheyenne River at Cherry Creek, S. Dak.

WATER QUALITY DATA

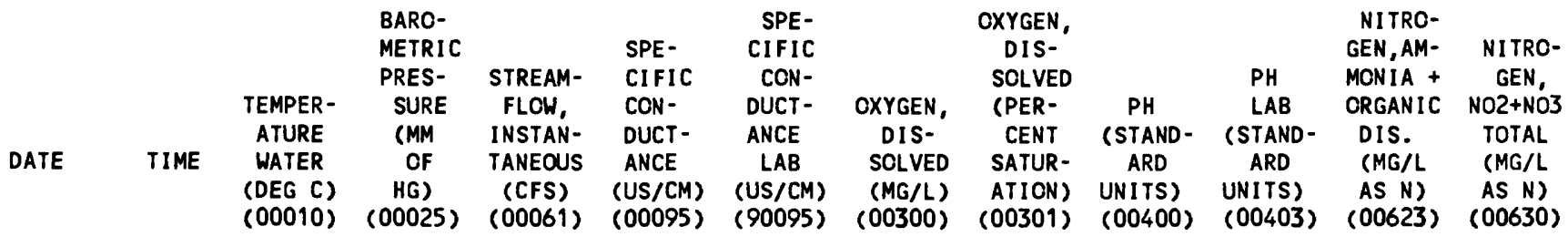

OCT 1984

JUN 1985

$10 . . .1100$

$19 . . .1000$

JUL

05... $\quad 1800$

$09 . .11145$

$17 . . . \quad 1545$

25... 1145

31... 1140

AUG

09... 1130

13... 1400

22... 1000

30... 1345

SEP

03... 0940

$09 . . . \quad 1030$

$19 . . . \quad 1115$

26... 1200

OCT

$03 \ldots 1200$

$10 \ldots \quad 1010$

$17 \ldots \quad 1130$

$24 \ldots \quad 1200$

$30 \ldots \quad 1000$

NOV

06... 1045

APR 1986

$11 \ldots \quad 1430$

22... 1245

29... $\quad 1230$

MAY

07... 1330

$14 \ldots .1130$

29... 1240 $\begin{array}{lll}11.5 & - & 171\end{array}$

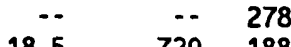

$\begin{array}{lll}18.5 & 720 & 188\end{array}$

27.0

27.0

26.5

25.0

$740 \quad 230$

$720 \quad 202$

$\begin{array}{ll}710 & 261\end{array}$

$720 \quad 294$

$\begin{array}{lll}17.0 & 721 \quad 367\end{array}$

19.5

20.5

23.0

26.0

701205

653148

653790

$\begin{array}{ll}710 & 273\end{array}$

$23.0 \quad 710 \quad 273$

$16.0 \quad 721 \quad 316$

$16.5 \quad 710 \quad 188$

$\begin{array}{lll}10.0 & 710 \quad 188\end{array}$

$10.0 \quad 630 \quad 178$

$\begin{array}{lll}5.0 & 720 \quad 162\end{array}$

$10.0 \quad 720 \quad 191$

$\begin{array}{lll}9.5 & 693 \quad 145\end{array}$

$\begin{array}{lll}6.0 & 720 \quad 162\end{array}$

$\begin{array}{lll}4.0 & 714 & 162\end{array}$

$21.0 \quad 697 \quad 1440$

$12.5 \quad--4890$

$9.0 \quad 712 \quad 8120$

$\begin{array}{lll}\ldots & - & 428\end{array}$

$\begin{array}{rrr}16.0 & 716 & 5240\end{array}$

$\begin{array}{llr}24.0 & 722 \quad 565\end{array}$

$\begin{array}{rrrr}-- & -- & -- & -- \\ 1830 & 2040 & -- & -- \\ & -- & 10.1 & 115 \\ 2160 & -- & 9.3 & 121 \\ 2250 & 2250 & 9.4 & 127 \\ 2110 & -- & 8.0 & 108 \\ 1980 & -- & 11.9 & 153 \\ 1950 & -- & 10.1 & 112 \\ & & & \\ 2140 & 2080 & 9.2 & 109 \\ 2110 & -- & 8.4 & 110 \\ 2020 & -- & 5.7 & 78 \\ 2170 & -- & 8.7 & 117 \\ & & & \\ 2170 & 2190 & 8.7 & 110 \\ 1960 & -- & 9.7 & 105 \\ 2250 & -- & 9.9 & 110 \\ 2410 & -- & 10.5 & 101 \\ & & & \\ 2800 & -- & 10.6 & 115 \\ 2520 & 2550 & 13.3 & 111 \\ 1840 & -- & 10.4 & 98 \\ 2420 & -- & 11.2 & 109 \\ 2340 & -- & 12.6 & 107 \\ & & & \\ 2330 & 2440 & 12.6 & 103\end{array}$

$\begin{array}{llll}2330 & 2440 & 12.6 & 103\end{array}$

-. $1260 \quad$..

$\begin{array}{ll}1000 & 1120 \\ 1040 & 1050\end{array}$

--
--
--

$--$

8.00

8.00

2070

956
2590 $-\cdot-$
929
2610 $\begin{array}{ll}-. & - \\ 7.9 & 86 \\ 7.7 & 98\end{array}$
7.90
7.90

7.74
8.40
8.40

8.60

8.50

8.50

8.50

8.40

7.90
8.30

8.30

8.20
8.10

8.20

8.40

7.90

8.40

8.20

8.10

8.10

8.30

7.60

7.80

7.

7.90
8.30
0.62

0.50

0.500

$\begin{array}{rr}-- & - \\ -- & - \\ 0.30 & <0.100\end{array}$ 
Table 37.--Chemical analyses of surface-water samples collected at Cheyenne River at Cherry Creek, S. Dak.--Cont inued

WATER QUALITY DATA

\begin{tabular}{|c|c|c|c|c|c|c|c|c|c|c|c|c|}
\hline ATE & $\begin{array}{c}\text { NITRO- } \\
\text { GEN, } \\
\text { NO2+NO3 } \\
\text { DIS- } \\
\text { SOLVED } \\
\text { (MG/L } \\
\text { AS N) } \\
(00631)\end{array}$ & $\begin{array}{l}\text { PHOS- } \\
\text { PHORUS, } \\
\text { TOTAL } \\
\text { (MG/L } \\
\text { AS P) } \\
(00665)\end{array}$ & $\begin{array}{c}\text { PHOS- } \\
\text { PHORUS, } \\
\text { DIS- } \\
\text { SOLVED } \\
\text { (MG/L } \\
\text { AS P) } \\
(00666)\end{array}$ & $\begin{array}{l}\text { CARBON, } \\
\text { ORGANIC } \\
\text { TOTAL } \\
\text { (MG/L } \\
\text { AS C) } \\
(00680)\end{array}$ & $\begin{array}{l}\text { CARBON, } \\
\text { ORGANIC } \\
\text { DIS- } \\
\text { SOLVED } \\
\text { (MG/L } \\
\text { AS C) } \\
(00681)\end{array}$ & $\begin{array}{l}\text { CYANIDE } \\
\text { TOTAL } \\
\text { (MG/L } \\
\text { AS CN) } \\
(00720)\end{array}$ & $\begin{array}{l}\text { CYANIDE } \\
\text { DIS- } \\
\text { SOLVED } \\
\text { (MG/L } \\
\text { AS CN) } \\
(00723)\end{array}$ & $\begin{array}{l}\text { SOLIDS, } \\
\text { RESIDUE } \\
\text { AT } 180 \\
\text { DEG. C } \\
\text { DIS- } \\
\text { SOLVED } \\
(M G / L) \\
(70300)\end{array}$ & $\begin{array}{l}\text { SOLIDS, } \\
\text { SUM OF } \\
\text { CONSTI- } \\
\text { TUENTS, } \\
\text { DIS- } \\
\text { SOLVED } \\
\text { (MG/L) } \\
(70301)\end{array}$ & $\begin{array}{l}\text { HARD- } \\
\text { NESS } \\
\text { (MG/L } \\
\text { AS } \\
\text { CACO3) } \\
(00900)\end{array}$ & $\begin{array}{l}\text { HARD- } \\
\text { NESS } \\
\text { NONCAR- } \\
\text { BONATE } \\
\text { (MG/L } \\
\text { AS } \\
\text { CACO3) } \\
\text { (95902) }\end{array}$ & $\begin{array}{l}\text { ALKA- } \\
\text { LINITY } \\
\text { LAB } \\
\text { (MG/L } \\
\text { AS } \\
\text { CACO3) } \\
(90410)\end{array}$ \\
\hline
\end{tabular}

OCT 1984

28 ....

JUN 1985

$10 . .$.

$19 . .$.

JUL

$05 .$.

$09 .$.

$25 \ldots$

$31 \ldots$

AUG

$09 . .$.

$13 . .$.

$22 \ldots$

$30 \ldots$

SEP

$03 . .$.

$09 .$.

$19 . .$.

26...

OCT

$03 . .$.

$10 . .$.

$17 . .$.

$24 \ldots$

$30 \ldots$

NOV

06...

APR 1986

$11 \ldots$

29...

0.094

MAY

$07 . .$.

$14 \ldots \quad 0.360$

$29 . .<<0.100$

0.730

0.460

$\begin{array}{ccr}6.4 & 4.6 & 0.010 \\ . & -. & \end{array}$

$\begin{array}{rr}0.01 & 1680 \\ \ldots & \ldots\end{array}$

$5.3^{--}<0.010$

4.7

$5.3<0.010$

-.

$0.01 \quad 1900$

1900

-.

-

$7.9 \quad 4.1<0.010$

$<0.0$

1750

--

-.

$\cdots$

-.

-.

$7.9 \quad 5.6<0.010$

$<0.01$

1880

$\overline{--}$

-.

5.6

-.

-.

-.

$-$

$3 . \overline{7}$

$3.2^{-}<0.010$

$<0.01$

2120

-.

$--$

-.

-.

-.

$\begin{array}{lll}4.6 & 3.7<0.010\end{array}$

$<0.01$

2030

$\begin{array}{ccr}7.8 & -- & <0.01 \\ -- & -- & -- \\ 6.8 & -- & <0.01\end{array}$

949

880

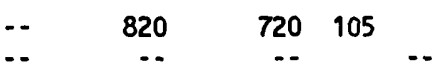

0.060

0.020

$<0.01 \quad 739 \quad 720 \quad 270$

$\begin{array}{cccc}-- & -- & -- & - \\ <01 & 739 & 720 & 270\end{array}$

250

950

$\begin{array}{rrr}-- & & - \\ 850 & 99 & \\ -- & & \ldots \\ - & & \ldots \\ -- & & --\end{array}$

-.

$800 \quad 97$

-.

$\begin{array}{ll}-- & - \\ -- & -\end{array}$

$1000 \quad 920 \quad 103$

$\begin{array}{ccccc}- & 1000 & 920 & 103 & \\ - & \ldots & - & & \ldots \\ - & \ldots & -. & \ldots \\ - & \ldots & \ldots & \end{array}$

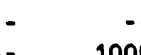

000

$890 \quad 141$

-.

$-$

-.

-

$\cdots$

$920 \quad 126$

39
37

6.8

0.030

5.3

$\begin{array}{cc}\cdots & \cdots \\ 5.4 & \cdots\end{array}$

$<0.01$

656

0
600
2100

240

$130 \quad 113$

-. 111

$130 \quad 104$

$730 \quad 161$ 
Table 37.--Chemical analyses of surface-water samples collected at

Cheyenne River at Cherry Creek, S. Dak. --Cont inued

WATER QUALITY DATA

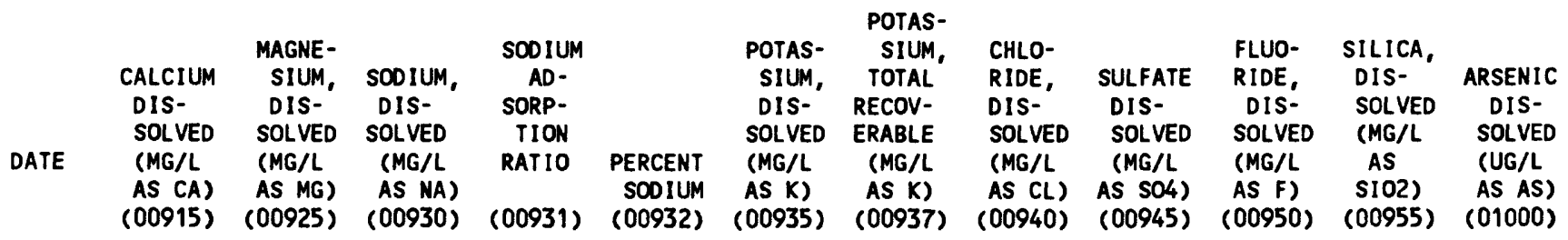

OCT 1984

JUN 1985

$10 . .$.

19.190

JUL

05.

17.

$25 .$.

31

AUG

$13 .$.

$22 .$.

$30 .$.

SEP

$09 . .$.

$19 .$.

26...

OCT

03... $230^{-\cdot} 110^{-\cdot} 240^{--}$

$17 . .$.

$24 \ldots$

$30 . .$.

NOV

$06 \ldots 220 \quad 120 \quad 230$

APR 1986

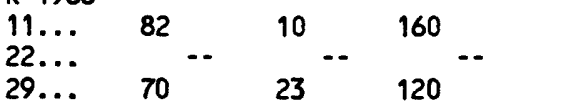

MAY

$07 . .$.

23

$14 \ldots 66$

$17^{-\cdots} 100$

$29 . . .220$

$84 \quad 280$

4

$\begin{array}{lll}-- & - & \\ 4 & 47 & 6.5 \\ & 40 & 12\end{array}$

7.1

$\begin{array}{llll}10 & 97 & & 1200 \\ -- & 14 & & 530 \\ -- & & -- & \end{array}$

$3^{-.}$

$--$

6.3

-. 11

420

0.30

7.9

8.0

$\begin{array}{lll}-- & 8.3 & 330^{--} \\ -- & 54^{--} & 1300^{-}\end{array}$

0.30

$-\cdot .1$
5.7

0.40

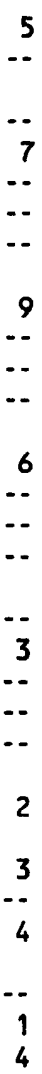


Table 37. - Chemical analyses of surface-water samples collected at Cheyenne River at Cherry Creek, S. Dak.--Cont inued

WATER QUALITY DATA

\begin{tabular}{|c|c|c|c|c|c|c|c|c|c|c|c|}
\hline$E$ & $\begin{array}{l}\text { ARSENIC } \\
\text { SUS- } \\
\text { PENDED } \\
\text { TOTAL } \\
\text { (UG/L } \\
\text { AS AS) } \\
\text { (01001) }\end{array}$ & $\begin{array}{l}\text { ARSENIC } \\
\text { TOTAL } \\
\text { (UG/L } \\
\text { AS AS) } \\
(01002)\end{array}$ & $\begin{array}{l}\text { BORON, } \\
\text { DIS- } \\
\text { SOLVED } \\
\text { (UG/L } \\
\text { AS B) } \\
\text { (01020) }\end{array}$ & $\begin{array}{l}\text { BORON, } \\
\text { TOTAL } \\
\text { RECOV- } \\
\text { ERABLE } \\
\text { (UG/L } \\
\text { AS B) } \\
(01022)\end{array}$ & $\begin{array}{l}\text { CADMIUM } \\
\text { DIS- } \\
\text { SOLVED } \\
\text { (UG/L } \\
\text { AS CD) } \\
(01025)\end{array}$ & $\begin{array}{l}\text { CADMIUM } \\
\text { TOTAL } \\
\text { RECOV- } \\
\text { ERABLE } \\
\text { (UG/L } \\
\text { AS CD) } \\
(01027)\end{array}$ & $\begin{array}{l}\text { CHRO- } \\
\text { MIUM, } \\
\text { DIS- } \\
\text { SOLVED } \\
\text { (UG/L } \\
\text { AS CR) } \\
\text { (01030) }\end{array}$ & $\begin{array}{l}\text { CHRO- } \\
\text { MIUM, } \\
\text { TOTAL } \\
\text { RECOV- } \\
\text { ERABLE } \\
\text { (UG/L } \\
\text { AS CR) } \\
(01034)\end{array}$ & $\begin{array}{l}\text { COPPER, } \\
\text { DIS- } \\
\text { SOLVED } \\
\text { (UG/L } \\
\text { AS CU) } \\
(01040)\end{array}$ & $\begin{array}{l}\text { COPPER, } \\
\text { SUS- } \\
\text { PENDED } \\
\text { RECOV- } \\
\text { ERABLE } \\
\text { (UG/L } \\
\text { AS CU) } \\
(01041)\end{array}$ & $\begin{array}{c}\text { COPPER, } \\
\text { TOTAL } \\
\text { RECOV- } \\
\text { ERABLE } \\
\text { (UG/L } \\
\text { AS CU) } \\
\text { (01042) }\end{array}$ \\
\hline
\end{tabular}

OCT 1984

$28 . .$.

JUN 1985

$10 . .$.

$19 .$.

JUL

05...

$09 . .$.

$17 . .$.

$25 \ldots$

$31 .$.

AUG

09...

$13 . .$.

22...

SEP

03...

$09 . .$.

$19 . .$.

26.

OCT

$03 . .$.

$10 . .$.

$17 . .$.

$24 \ldots$

$30 . .$.

NOV

$06 \ldots$

APR 1986

$11 \ldots$

$22 .$.

MAY

$07 . .$.

$14 \ldots$

$29 .$.

\begin{tabular}{|c|c|c|c|}
\hline$\cdots$ & -- & -- & - \\
\hline 11 & 16 & 280 & 300 \\
\hline-- & -- & - & -- \\
\hline- & -. & -. & -- \\
\hline$\cdots$ & -- & 360 & 400 \\
\hline-- & -- & -- & - \\
\hline- & -- & -. & - \\
\hline - & -- & -- & - \\
\hline 3 & 12 & 330 & 340 \\
\hline - & $\cdots$ & -- & -- \\
\hline$-\cdot$ & $\cdots$ & $-\cdot$ & -- \\
\hline-- & -- & -- & $\cdots$ \\
\hline 9 & 15 & 350 & 340 \\
\hline$\cdots$ & $\cdots$ & -- & -- \\
\hline$\cdots$ & $\cdots$ & $-\cdot$ & - \\
\hline$\cdots$ & $\cdots$ & -- & -- \\
\hline-- & -- & -- & -- \\
\hline 2 & 5 & 330 & 340 \\
\hline -. & -- & - & -- \\
\hline$\cdots$ & -- & -- & $\cdots$ \\
\hline-- & $-\cdot$ & -- & - \\
\hline 2 & 4 & 320 & 370 \\
\hline-- & -- & -- & -. \\
\hline-- & -- & $\cdots$ & - \\
\hline$\cdots$ & $\cdots$ & -- & -- \\
\hline-- & -. & -. & -- \\
\hline-- & -- & - & $\cdots$ \\
\hline-- & $\cdots$ & $-\cdot$ & $\cdots$ \\
\hline
\end{tabular}

$\begin{array}{lll}1 & 1 \\ -- & - & - \\ -- & - & - \\ -- & - & - \\ -- & - & -\end{array}$

10

20

..

11

$<1 \quad<10$

10

$--$

-

$<1$

$<1<10$

$\begin{array}{ccc}<1 & <1 & <10 \\ -- & -- & -- \\ -- & -- & -- \\ -- & -- & --\end{array}$

$-$

$<1<1<10<10$

$\begin{array}{lll}<1 & <1 & <10 \\ - & - & -\end{array}$

-.

$\begin{array}{ll}-- & - \\ -- & <\end{array}$

$<1<1<10$

$<10 \quad 10$

$10 \quad 1$

$\because$

$-$

$\cdot$

$\because$

5
--

3

$\begin{array}{rr}-- & \cdots \\ 4 & 5\end{array}$

$-$

$\cdots$

79

$<1 \quad--$

$\cdot$

$-\cdot$

3

$\begin{array}{ll}-- & -- \\ <1 & -- \\ <9 & --\end{array}$

--
-- 
Table 37.--Chemical analyses of surface-water samples collected at Cheyenne River at Cherry Creek, S. Dak.--Cont inued

WATER QUALITY DATA

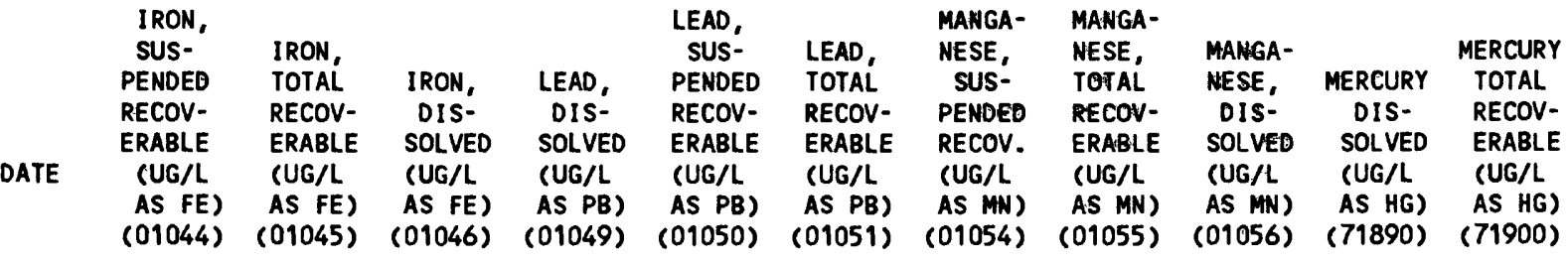

\begin{tabular}{|c|c|c|c|c|c|c|c|c|c|c|c|}
\hline $\begin{array}{l}\text { OCT } 1984 \\
28 . . \\
\text { JUN } 1985\end{array}$ & $\cdots$ & $\cdots$ & $\cdots$ & $\cdots$ & $-\cdot$ & -- & $\cdots$ & $\cdots$ & $\cdots$ & $\cdots$ & $\cdots$ \\
\hline $10 \ldots$ & 1400 & 1400 & 20 & 1 & $\cdots$ & 8 & -- & 780 & 10 & 0.1 & 0.30 \\
\hline JUL $19 . .$. & $\cdots$ & -- & $-\cdot$ & -- & -- & -- & $-\cdot$ & $\cdots$ & $\cdots$ & -. & -- \\
\hline $05 \ldots$ & -- & - & $\cdots$ & $\cdots$ & -. & -- & -. & -- & $\cdots$ & -. & \\
\hline $09 \ldots$ & 3100 & 3100 & 20 & 4 & -- & $<1$ & -- & 160 & $<10$ & $<0.1$ & $<0.10$ \\
\hline $17 \ldots$ & -. & - & -- & -- & -- & -- & -- & -- & -. & -. & $\cdots$ \\
\hline $25 \ldots$ & $-\cdot$ & $\cdots$ & -- & $\cdots$ & $-\cdot$ & $\cdots$ & $\cdots$ & $\cdots$ & $\cdots$ & -- & $-\cdot$ \\
\hline $31 \ldots$ & $\cdots$ & $\cdots$ & $-\cdot$ & $\cdots$ & -- & -- & $\cdots$ & -- & $\cdots$ & -- & . \\
\hline AUG & & & & & & & & & & & \\
\hline $09 \ldots$ & 530 & 560 & 30 & $<1$ & $\cdots$ & 4 & 180 & 190 & 10 & $<0.1$ & $<0.10$ \\
\hline $13 \ldots$ & -- & -- & $\cdots$ & $\because$ & -- & $\cdots$ & $\cdots$ & -- & -- & $\cdots$ & $\cdots$ \\
\hline $22 \ldots$ & 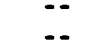 & $\cdots$ & $\cdots$ & $\cdots$ & $\cdots$ & $\cdots$ & $\cdots$ & $\cdots$ & $\cdots$ & $\because$ & - \\
\hline$\underset{\text { SEP }}{30 \ldots}$ & $\cdots$ & $\cdots$ & $\cdots$ & -- & $\cdots$ & $\cdots$ & $\cdots$ & $\cdots$ & $\cdots$ & $\cdots$ & \\
\hline $\begin{array}{l}\text { SEP } \\
03 \ldots\end{array}$ & 3000 & 3000 & 30 & $<1$ & -- & 3 & -- & 150 & $<10$ & $<0.1$ & $<0.10$ \\
\hline $09 .$. & -- & -- & $\cdots$ & $\cdots$ & $\cdots$ &.- & -- & -- & -- & -- & $\cdots$ \\
\hline $19 .$. & $\cdots$ & $\cdots$ & -- & - & $\cdots$ & $\cdots$ & -- & $\cdots$ & $\cdots$ & -- & \\
\hline${ }_{\text {OCT }}^{26 . . .}$ & -- & $\cdots$ & $\cdots$ & -- & -- & $\cdots$ & -- & $\cdots$ & -- & -- & \\
\hline $03 \ldots$ & $\cdots$ & $\cdots$ & $\cdots$ & $\cdots$ & -- & - & - & $\cdots$ & $\cdots$ & $\cdots$ & -. \\
\hline $10 \ldots$ & 870 & 900 & 30 & 2 & 0 & 2 & 40 & 90 & 50 & $<0.1$ & $<0.10$ \\
\hline $17 \ldots$ & -- & $-\cdot$ & $\cdots$ & $\cdots$ & $\cdots$ & -- & -- & $\cdots$ & $\cdots$ & -. & - \\
\hline $24 \ldots$ & $\cdots$ & -- & $\cdots$ & $\cdots$ & $\because$ & $\cdots$ & $\cdots$ & $-\cdot$ & $\cdots$ & -- & \\
\hline $30 \ldots$ & $\cdots$ & -- & $\cdots$ & $\cdot-$ & $\cdots$ & $\cdots$ & $\cdots$ & $\cdots$ & $\cdots$ & -- & \\
\hline $06 \ldots$ & 1900 & 1900 & 40 & $<1$ & $\ldots$ & $?$ & 70 & 110 & 10 & $=09$ & 0.20 \\
\hline APR 1986 & 1780 & 1800 & 40 & 1 & 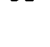 & $c$ & ru & 170 & 40 & 8.1 & $0 .<0$ \\
\hline $11 \ldots$ & -- & $-\cdot$ & 7 & $\cdots$ & $-\cdot$ & $\because$ & $\cdots$ & $\cdot-$ & 2 & $<0.1$ & $\cdots$ \\
\hline $22 \ldots$ & $\cdots$ & $\cdots$ & $\ddot{m}$ & $-\cdot$ & $-\cdot$ & $\cdots$ & -- & $\cdots$ & $\because$ & $\cdots$ & $\ldots$ \\
\hline $29 \ldots$ & -- & -- & 20 & $\cdots$ & $\cdots$ & -- & -- & -- & 2 & $<0.1$ & \\
\hline MAY & & & & & & & & & & & \\
\hline $\begin{array}{l}07 . . \\
14 \ldots\end{array}$ & $\cdots$ & - & $\ldots$ & 1 & $\ldots$ & 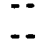 & 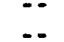 & $\cdots$ & $-\overline{1}$ & $\ddot{4}$ & $\because$ \\
\hline 29.. & -. & -. & 20 & $<5$ & -- & -. & - & -. & 20 & $<0.1$ & $\because$ \\
\hline
\end{tabular}


Table 37.-- Chemical analyses of surface-water samples collected at Cheyenne River at Cherry Creek, S. Dak.--Cont inued

\begin{tabular}{|c|c|c|c|c|c|c|c|c|c|c|}
\hline \multicolumn{11}{|c|}{ WATER QUALITY DATA } \\
\hline $\begin{array}{l}\text { SILVER, } \\
\text { DIS- } \\
\text { SOLVED } \\
\text { (UG/L } \\
\text { AS AG) } \\
(01075)\end{array}$ & $\begin{array}{l}\text { SILVER, } \\
\text { TOTAL } \\
\text { RECOV- } \\
\text { ERABLE } \\
\text { (UG/L } \\
\text { AS AG) } \\
(01077)\end{array}$ & $\begin{array}{l}\text { ZINC, } \\
\text { DIS- } \\
\text { SOLVED } \\
\text { (UG/L } \\
\text { AS ZN) } \\
(01090)\end{array}$ & $\begin{array}{l}\text { ZINC, } \\
\text { SUS- } \\
\text { PENDED } \\
\text { RECOV- } \\
\text { ERABLE } \\
\text { (UG/L } \\
\text { AS ZN) } \\
(01091)\end{array}$ & $\begin{array}{l}\text { ZINC, } \\
\text { TOTAL } \\
\text { RECOV- } \\
\text { ERABLE } \\
\text { (UG/L } \\
\text { AS ZN) } \\
(01092)\end{array}$ & $\begin{array}{l}\text { ANTI - } \\
\text { MONY, } \\
\text { DIS- } \\
\text { SOLVED } \\
\text { (UG/L } \\
\text { AS SB) } \\
(01095)\end{array}$ & $\begin{array}{l}\text { ALUM- } \\
\text { INUM, } \\
\text { TOTAL } \\
\text { RECOV- } \\
\text { ERABLE } \\
\text { (UG/L } \\
\text { AS AL) } \\
(01105)\end{array}$ & $\begin{array}{l}\text { ALUM- } \\
\text { INUM, } \\
\text { DIS- } \\
\text { SOLVED } \\
\text { (UG/L } \\
\text { AS AL) } \\
(01106)\end{array}$ & $\begin{array}{l}\text { ALUM- } \\
\text { INUM, } \\
\text { SUS- } \\
\text { PENDED } \\
\text { RECOV. } \\
\text { (UG/L } \\
\text { AS AL) } \\
(01107)\end{array}$ & $\begin{array}{l}\text { SELE- } \\
\text { NIUM, } \\
\text { DIS- } \\
\text { SOLVED } \\
\text { (UG/L } \\
\text { AS SE) } \\
(01145)\end{array}$ & $\begin{array}{l}\text { SELE- } \\
\text { NIUM, } \\
\text { SUS- } \\
\text { PENDED } \\
\text { TOTAL } \\
\text { (UG/L } \\
\text { AS SE) } \\
(01146)\end{array}$ \\
\hline
\end{tabular}

\section{OCT 1984}

$28 . .$.

JUN 1985

$10 . .$.

$19 .$.

JUL

$05 . .$.

$09 . .$.

$17 . .$.

$25 \ldots$

$31 \ldots$

AUG

$09 . .$.

$13 . .$.

22...

$30 .$.

SEP

$03 . .$.

$09 . .$.

$19 . .$.

26...

OCT

03...

$10 .$.

$17 . .$.

$24 . .$.

$30 . .$.

NOV

$06 . .$.

APR 1986

$11 . .$.

$22 .$.

$29 . .$.

MAY

$07 .$. .

$14 \ldots$

$29 . .$.

$\begin{array}{lll}1 & 1 & \\ -- & -- & \\ -- & -. & \\ <1 & <1 & <10 \\ -- & -- & - \\ -- & -- & - \\ -- & -. & -\end{array}$

10
--
--
$<10$
$\cdots-$
--
-

60

7

$-$

$<1 \quad<10$

$\begin{array}{lll}-- & -. & - \\ -. & -. & - \\ -. & -. & -\end{array}$

-- $\quad 30$

30

$\begin{array}{ll}-- & - \\ -- & --\end{array}$

$<1<1 \quad 10$

20

$20 \quad 30$

30

$\begin{array}{lll}-- & -- & -- \\ -- & -- & --\end{array}$

$\begin{array}{ll}-- & -. \\ -- & -.\end{array}$

$-$

-.

o 10

10

$<1<1 \quad 10$

-. -- $\quad$--

$\begin{array}{lll}-- & - & - \\ -- & -- & -\end{array}$

$<1<1 \quad 10$

10

$\begin{array}{ccc}-- & -. & <3 \\ -. & -. & -- \\ -- & -- & 8\end{array}$

$\begin{array}{lll}-. & -- & 1 \\ -- & -- & - \\ -. & -. & <1\end{array}$

$3800 \quad 20 \quad 3800$

30

$\begin{array}{lll}-- & -. & -- \\ <1 & -- & 10\end{array}$

$\begin{array}{rrr}<1 & -- & 10 \\ -- & -- & <10\end{array}$

$\begin{array}{ll}-- & -- \\ -- & -- \\ -- & --\end{array}$ 
Table 37.--Chemical analyses of surface-water samples collected at Cheyenne River at Cherry Creek, S. Dak.--Cont inued

WATER QUALITY DATA

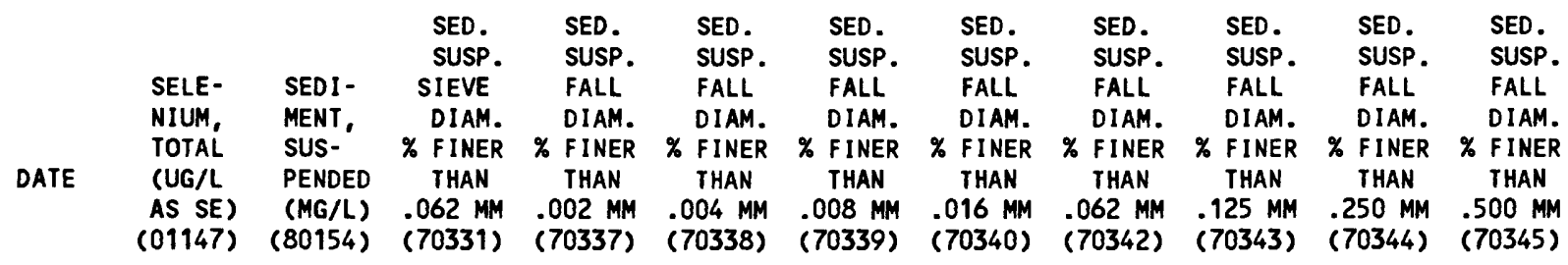

OCT 1984

$28 . .$.

JUN 1985

$10 . .$.

$19 . .$.

JUL

$05 .$. .

$09 .$.

$17 . .$.

$25 .$.

$31 \ldots$

AUG

$09 .$.

$13 . .$.

$22 \ldots$

$30 . .$.

SEP

$03 . .$.

$09 .$. .

$19 . .$.

26 ...

OCT

03...

$10 . .$.

$17 . .$.

$24 . .$.

$30 . .$.

NOV

$06 . .$.

APR 1986

$11 . .$.

22...

$29 . .$.

MAY

$07 . .$.

$14 \ldots$

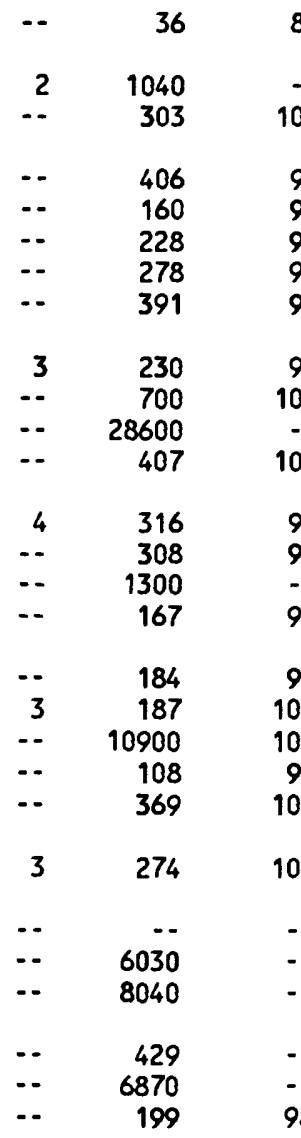

\section{9}

100

20

23

97

99

99
98

99

48

53

-. $\quad 28$

33

$\begin{array}{ll}-- & - \\ 76 & 88\end{array}$

70

68

88
79
75
57

--

59

-.

33

35

85

$29 .$.

99
100

100

100

80

62

68

87

99
99

99

99

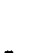

--

81

89

96

98

94

98
80

$36 \quad 55$

84

--

--

$--$

99

$41 \quad 70$

--

82

70

91

97

99

80

-.

$--$

95

95
98
96

-.

100

--

$-$

98

99

97

98

00

90

99

$-$

-.

-.

-.

9

$\begin{array}{ll}-- \\ - & 51\end{array}$

61

70

77

55

63

69

$\because-$

-.

--

98 
Table 37.-- Chemical analyses of surface-water samples collected at Cheyenne River at Cherry Creek, S. Dak.--Cont inued

WATER QUALITY DATA

\begin{tabular}{|c|c|c|c|c|c|c|c|c|c|c|c|}
\hline DATE & TIME & $\begin{array}{l}\text { TEMPER- } \\
\text { ATURE } \\
\text { WATER } \\
\text { (DEG C) } \\
(00010)\end{array}$ & $\begin{array}{l}\text { BARO- } \\
\text { METRIC } \\
\text { PRES- } \\
\text { SURE } \\
\text { (MM } \\
\text { OF } \\
\text { HG) } \\
(00025)\end{array}$ & $\begin{array}{l}\text { STREAM- } \\
\text { FLOW, } \\
\text { INSTAN- } \\
\text { TANEOUS } \\
\text { (CFS) } \\
\text { (00061) }\end{array}$ & $\begin{array}{l}\text { SPE- } \\
\text { CIFIC } \\
\text { CON- } \\
\text { DUCT- } \\
\text { ANCE } \\
\text { (US/CM) } \\
(00095)\end{array}$ & $\begin{array}{c}\text { SPE- } \\
\text { CIFIC } \\
\text { CON- } \\
\text { DUCT- } \\
\text { ANCE } \\
\text { LAB } \\
\text { (US/CM) } \\
(90095)\end{array}$ & $\begin{array}{c}\text { OXYGEN, } \\
\text { DIS- } \\
\text { SOLVED } \\
\text { (MG/L) } \\
(00300)\end{array}$ & $\begin{array}{l}\text { OXYGEN, } \\
\text { DIS- } \\
\text { SOLVED } \\
\text { (PER- } \\
\text { CENT } \\
\text { SATUR- } \\
\text { ATION) } \\
\text { (O0301) }\end{array}$ & $\begin{array}{c}\text { PH } \\
\text { (STAND- } \\
\text { ARD } \\
\text { UNITS) } \\
(00400)\end{array}$ & $\begin{array}{c}\text { PH } \\
\text { LAB } \\
\text { (STAND- } \\
\text { ARD } \\
\text { UNITS) } \\
\text { (00403) }\end{array}$ & $\begin{array}{l}\text { NITRO- } \\
\text { GEN, } \\
\text { TOTAL } \\
\text { (MG/L } \\
\text { AS N) } \\
(00600)\end{array}$ \\
\hline
\end{tabular}

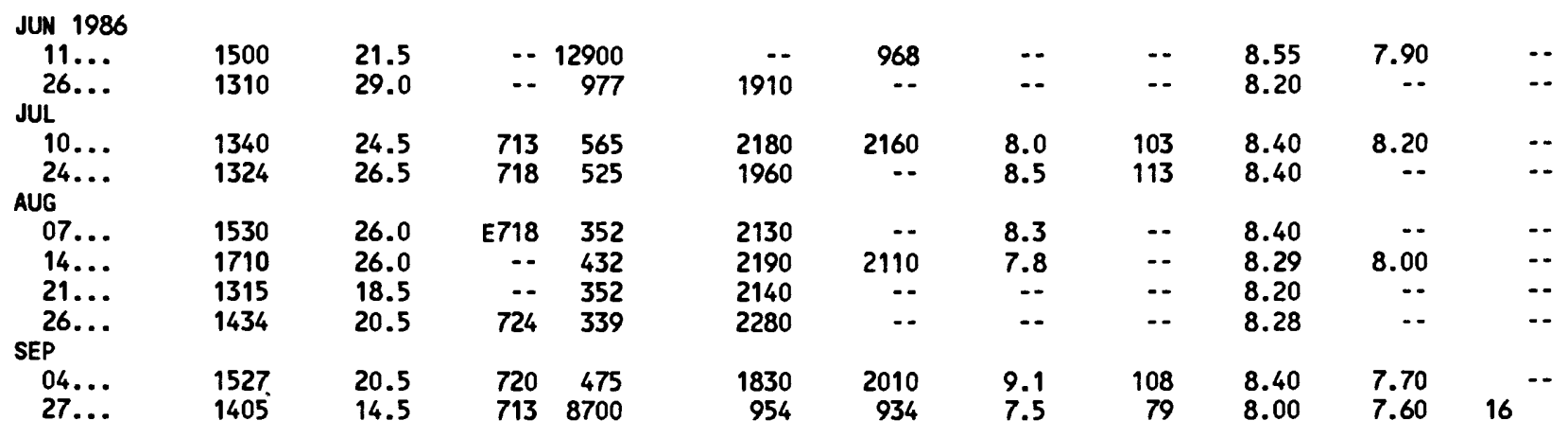

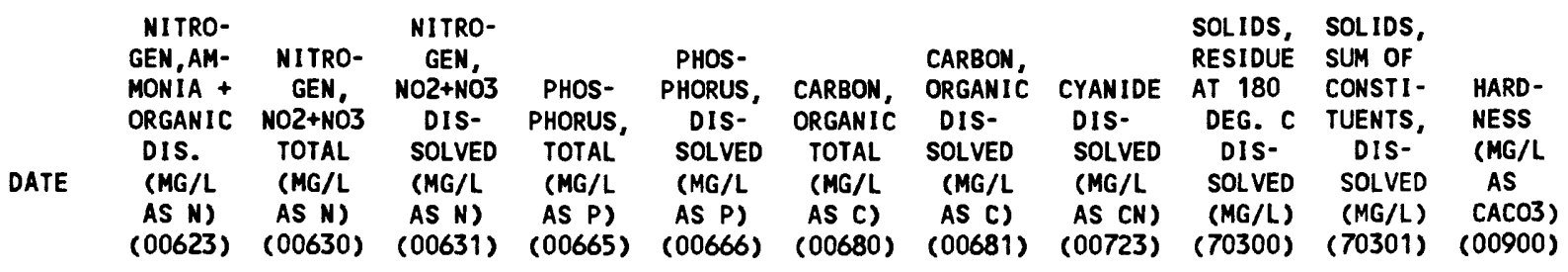

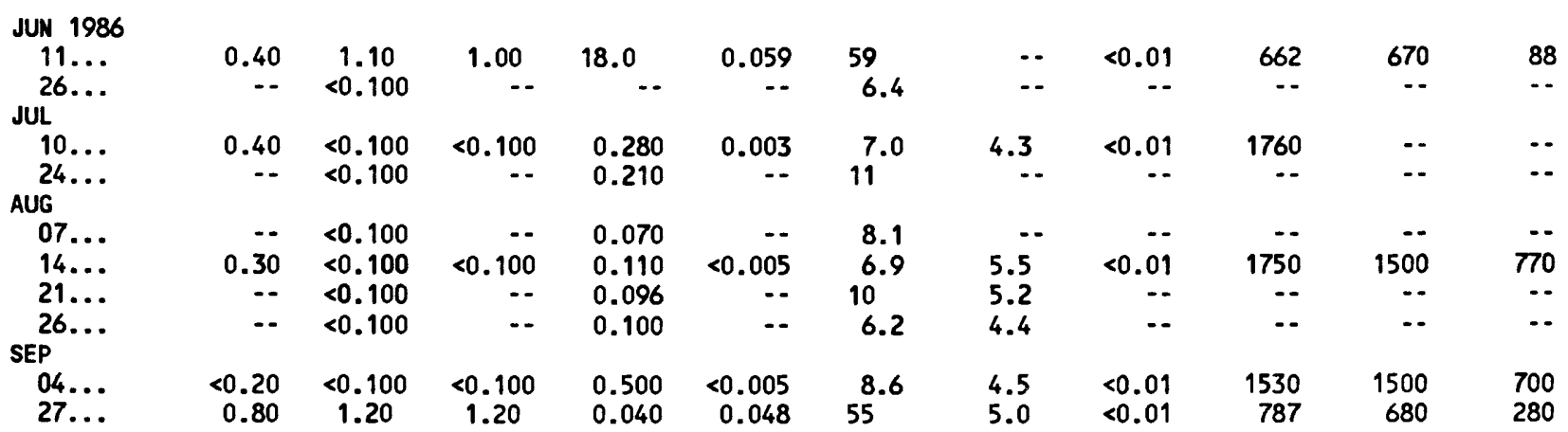


Table 37.-- Chemical analyses of surface-water samples collected at cheyenne River at Cherry Creek, S. Dak.--Cont inued

WATER QUALITY DATA

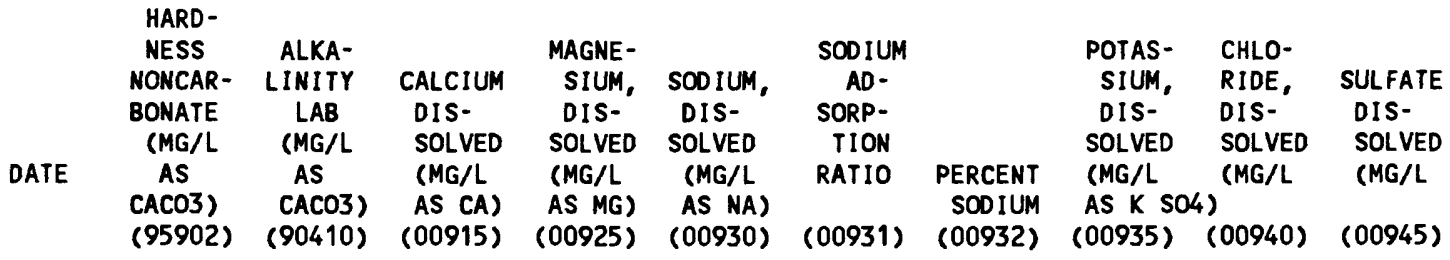

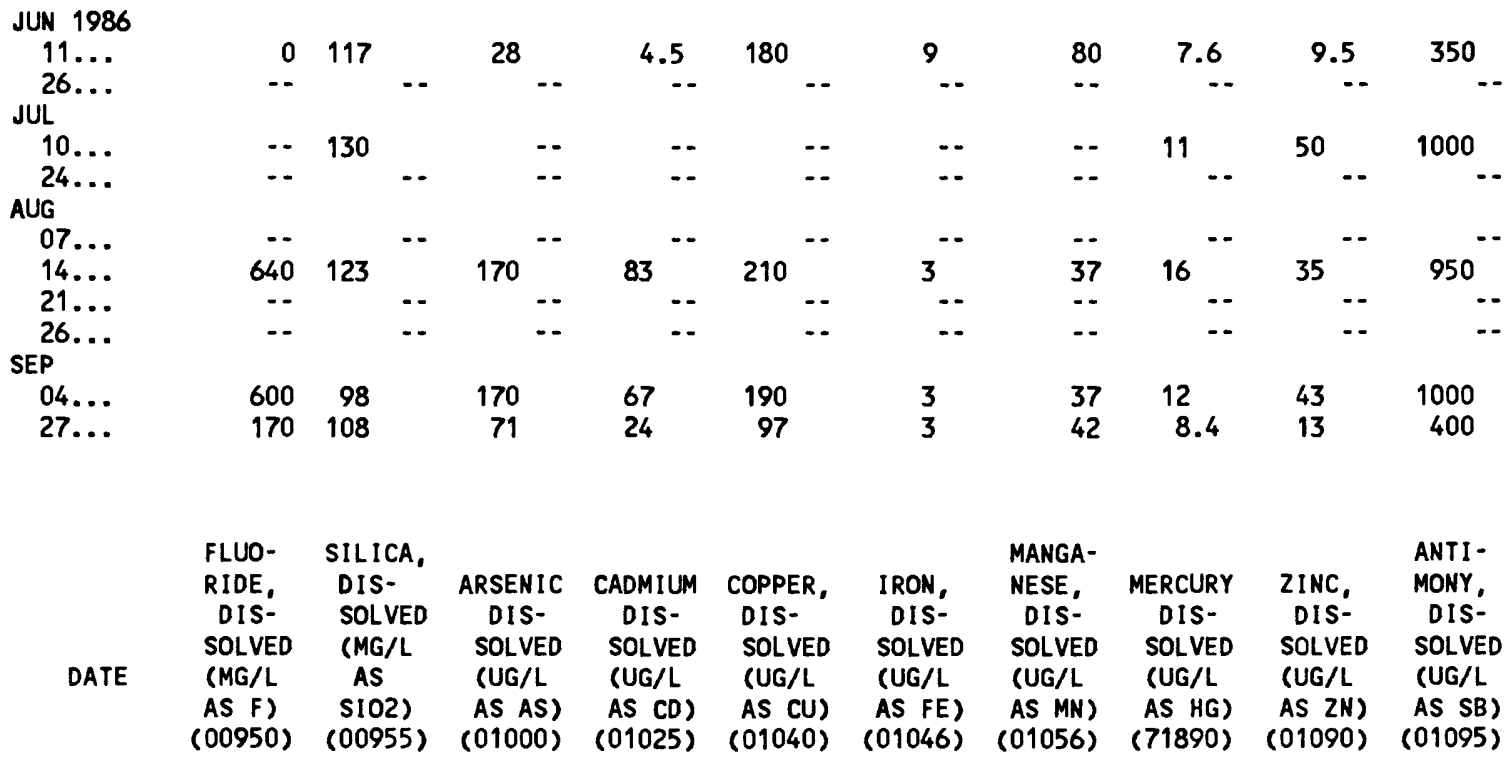

\begin{tabular}{|c|c|c|c|c|c|c|c|c|c|c|}
\hline JUN 1986 & & & & & & & & & & \\
\hline $11 \ldots$ & 0.70 & 21 & 4 & $<1$ & 7 & 14 & 1 & $<0.1$ & 4 & 1 \\
\hline 26 & - & -. & $\cdots$ & $\cdots$ & $\cdots$ & -- & -- & -. & $\cdots$ & -- \\
\hline JUL & & & & & & & & & & \\
\hline $10 \ldots$ & 0.40 & 6.5 & 3 & $<1$ & 2 & 20 & $<10$ & $<0.1$ & $<10$ & $<1$ \\
\hline $24 \ldots$ & -- & -- & -- & - &.- & -- & -- & -- & -- & $\cdots$ \\
\hline AUG & & & & & & & & & & \\
\hline $07 \ldots$ & -- & -- & -- & - & $=$ & -- & -- & -- & -- & -- \\
\hline $14 \ldots$ & 0.50 & 5.0 & 3 & $<1$ & 3 & 20 & 10 & $<0.1$ & $<10$ & $<1$ \\
\hline $21 \ldots$ & -- & $\cdots$ & $\cdots$ & $\cdots$ & $\cdots$ & $\cdots$ & -- & -- & -- & - \\
\hline $26 \ldots$ & -- & -- & $\cdots$ & $\cdots$ & -- & -- & $\cdots$ & $\cdots$ & $\cdots$ & - \\
\hline SEP & & & & & & & & & & \\
\hline $04 \ldots$ & 0.50 & 5.1 & 4 & $<1$ & 1 & 30 & $<10$ & $<0.1$ & 10 & $<1$ \\
\hline $27 \ldots$ & 0.50 & 5.2 & 4 & $<1$ & 3 & 7 & 1 & $<0.1$ & 12 & $<1$ \\
\hline
\end{tabular}


Table 37.-- Chemical analyses of surface-water samples collected at Cheyenne River at Cherry Creek, S. Dak.--Cont inued

WATER QUALITY DATA

\begin{tabular}{|c|c|c|c|c|c|c|c|c|c|c|}
\hline \multicolumn{11}{|c|}{ WATER QUALITY DATA } \\
\hline DATE & $\begin{array}{l}\text { SEDI- } \\
\text { MENT, } \\
\text { SUS- } \\
\text { PENDED } \\
\text { (MG/L) } \\
(80154)\end{array}$ & $\begin{array}{l}\text { SED. } \\
\text { SUSP. } \\
\text { SIEVE } \\
\text { DIAM. } \\
\times \text { FINER } \\
\text { THAN } \\
.062 \text { MM } \\
(70331)\end{array}$ & $\begin{array}{l}\text { SED. } \\
\text { SUSP. } \\
\text { FALL } \\
\text { DIAM. } \\
\times \text { FINER } \\
\text { THAN } \\
.002 \mathrm{MM} \\
(70337)\end{array}$ & $\begin{array}{l}\text { SED. } \\
\text { SUSP. } \\
\text { FALL } \\
\text { DIAM. } \\
\text { \% FINER } \\
\text { THAN } \\
.004 \text { MM } \\
(70338)\end{array}$ & $\begin{array}{l}\text { SED. } \\
\text { SUSP. } \\
\text { FALL } \\
\text { DIAM. } \\
\text { \% FINER } \\
\text { THAN } \\
.008 \mathrm{MM} \\
(70339)\end{array}$ & $\begin{array}{l}\text { SED. } \\
\text { SUSP. } \\
\text { FALL } \\
\text { DIAM. } \\
\times \text { FINER } \\
\text { THAN } \\
.016 \text { MM } \\
(70340)\end{array}$ & $\begin{array}{l}\text { SED. } \\
\text { SUSP. } \\
\text { FALL } \\
\text { DIAM. } \\
\text { \% FINER } \\
\text { THAN } \\
.062 \text { MM } \\
\text { (70342) }\end{array}$ & $\begin{array}{l}\text { SED. } \\
\text { SUSP. } \\
\text { FALL } \\
\text { DIAM. } \\
\text { \% FINER } \\
\text { THAN } \\
.125 \mathrm{MM} \\
\text { (70343) }\end{array}$ & $\begin{array}{l}\text { SED. } \\
\text { SUSP. } \\
\text { FALL } \\
\text { DIAM. } \\
\times \text { FINER } \\
\text { THAN } \\
.250 \mathrm{MM} \\
(70344)\end{array}$ & $\begin{array}{l}\text { SED. } \\
\text { SUSP. } \\
\text { FALL } \\
\text { DIAM. } \\
\% \text { FINER } \\
\text { THAN } \\
.500 \text { MM } \\
(70345)\end{array}$ \\
\hline N 1986 & & & & & & & & & & \\
\hline $11 \ldots$ & 27600 & -- & 64 & 74 & 82 & 89 & 99 & 100 & $\cdots$ & $\cdots$ \\
\hline${ }_{\mathrm{J}}^{26 \ldots}$ & 188 & 95 & -- & -- & -- & -- & $\cdots$ & $\cdots$ & -- & $\cdots$ \\
\hline $10 \ldots$ & 517 & 99 & 66 & 78 & 90 & 95 & $\cdots$ & $\cdots$ & $\cdots$ & -- \\
\hline & 201 & 98 & $\cdots$ & - & $\cdots$ & $\cdots$ & $\cdots$ & -- & - & -- \\
\hline $07 \ldots$ & 160 & 99 & $\cdots$ & $\cdots$ & $\cdots$ & $\cdots$ & -. & -. & $\cdots$ & -. \\
\hline $14 \ldots$ & 432 & 99 & 63 & 76 & 89 & 95 & $\cdots$ & -- & $\cdots$ & $\cdots$ \\
\hline $21 \ldots$ & 159 & 100 & $\cdots$ & $\cdots$ & $\cdots$ & $\cdots$ & $\cdots$ & $\cdots$ & $\cdots$ & -- \\
\hline $26 \ldots$ & 71 & 99 & $\cdots$ & $\cdots$ & $\cdots$ & - & $\cdots$ & $\cdots$ & - & -- \\
\hline $04 \ldots$ & $\begin{array}{r}504 \\
17200\end{array}$ & $\begin{array}{l}99 \\
\ldots-\end{array}$ & 74 & $\begin{array}{l}87 \\
63\end{array}$ & 70 & 99 & $\ddot{0 .}$ & $\overline{0-}$ & $\ddot{0}$ & $\ddot{n}$ \\
\hline $27 \ldots$ & 17200 & $\cdots$ & 51 & 63 & 72 & 79 & 91 & 95 & 98 & 100 \\
\hline
\end{tabular}


Table 38.--Chemical analyses of surface-water samples collected at 14 miscellaneous sites

\begin{tabular}{|c|c|c|c|c|c|c|c|c|c|c|}
\hline TIME & $\begin{array}{c}\text { TEMPER - } \\
\text { ATURE } \\
\text { WATER } \\
\text { (DEG C) } \\
(00010)\end{array}$ & $\begin{array}{l}\text { BARO- } \\
\text { METRIC } \\
\text { PRES- } \\
\text { SURE } \\
\text { (MM } \\
\text { OF } \\
\text { HG) } \\
(00025)\end{array}$ & $\begin{array}{l}\text { STREAM- } \\
\text { FLOW, } \\
\text { INSTAN- } \\
\text { TANEOUS } \\
\text { (CFS) } \\
\text { (00061) }\end{array}$ & $\begin{array}{l}\text { SPE- } \\
\text { CIFIC } \\
\text { CON- } \\
\text { DUCT- } \\
\text { ANCE } \\
\text { (US/CM) } \\
(00095)\end{array}$ & $\begin{array}{c}\text { SPE- } \\
\text { CIFIC } \\
\text { CON- } \\
\text { DUCT- } \\
\text { ANCE } \\
\text { LAB } \\
\text { (US/CM) } \\
(90095)\end{array}$ & $\begin{array}{c}\text { OXYGEN, } \\
\text { DIS- } \\
\text { SOLVED } \\
\text { (MG/L) } \\
(00300)\end{array}$ & $\begin{array}{c}\text { OXYGEN, } \\
\text { OIS- } \\
\text { SOLVED } \\
\text { (PER- } \\
\text { CENT } \\
\text { SATUR- } \\
\text { ATION) } \\
\text { (OO3O1) }\end{array}$ & $\begin{array}{c}\text { PH } \\
\text { (STAND- } \\
\text { ARD } \\
\text { UNITS) } \\
\text { (00400) }\end{array}$ & $\begin{array}{c}\text { PH } \\
\text { LAB } \\
\text { (STAND- } \\
\text { ARD } \\
\text { UNITS) } \\
\text { (00403) }\end{array}$ & $\begin{array}{l}\text { NITRO- } \\
\text { GEN, } \\
\text { TOTAL } \\
\text { (MG/L } \\
\text { AS N) } \\
(00600)\end{array}$ \\
\hline
\end{tabular}

444121101174001 CHEYENNE RIVER AT CARLIN FLAT, S.D. (LAT 4441 21N LONG 10117 40W)

\begin{tabular}{|c|c|c|c|c|c|c|c|c|c|c|c|}
\hline \multicolumn{12}{|l|}{ AUG 1986} \\
\hline $14 \ldots$ & 1425 & 24.5 & 716 & 503 & 2130 & 2110 & 7.6 & & 8.15 & 8.10 & $\cdots$ \\
\hline $21 \ldots$ & 1230 & 18.5 & $\cdots$ & 441 & 2140 & -- & -- & $\cdots$ & 8.17 & -. & $\cdots$ \\
\hline $26 \ldots$ & 1700 & 21.5 & $\cdots$ & 332 & 2320 & $\cdots$ & $\cdots$ & $\cdots$ & 8.30 & $\cdots$ & -- \\
\hline $\begin{array}{l}\text { SEP } \\
04 .\end{array}$ & & & & & & & & & & & \\
\hline 04. & 1258 & 20.5 & 723 & 494 & 2100 & -- & 9.0 & 106 & 8.36 & $\cdots$ & $\cdots$ \\
\hline
\end{tabular}

442134103443501 GOLD RUN BELOW HOMESTAKE DISCHARGE (LAT $442134 \mathrm{~N}$ LONG $1034435 \mathrm{~W}$ )

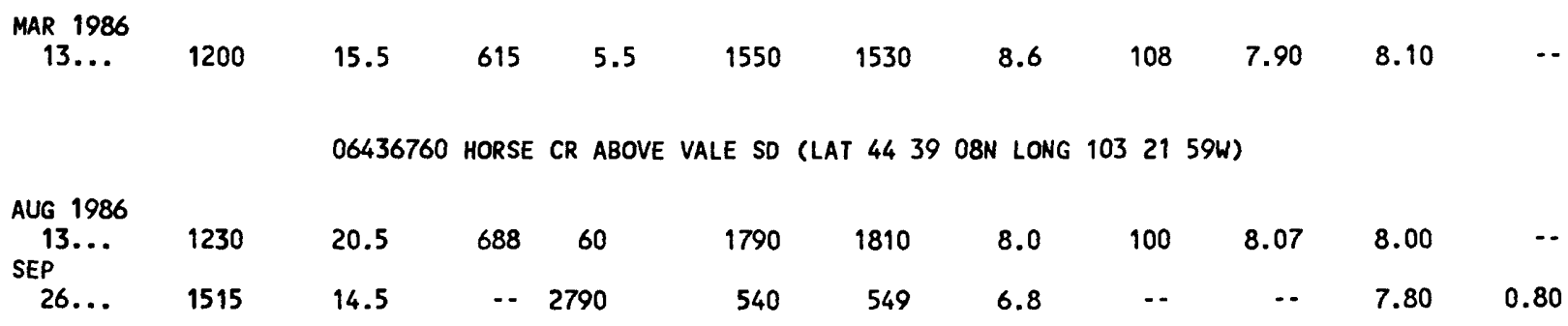

442134103441901 WHITEWOOD CREEK ABOVE GOLD RUN (LAT $442134 \mathrm{~N}$ LONG 10344 19W)

\begin{tabular}{|c|c|c|c|c|c|c|c|c|c|}
\hline $13 .$. & 1430 & 15.0 & 620 & 9.3 & 430 & 437 & 11.7 & 143 & 8.40 \\
\hline
\end{tabular}

06436170 WHITEWOOD CREEK AT DEADWOOD (LAT $442248 \mathrm{~N}$ LONG 10343 25W)

$\begin{array}{cccccccccccc}\text { MAR } 1986 & & & & & & & & & & \\ 13 . . . & 1730 & 7.0 & 630 & 25 & -- & 799 & \ldots & -. & -. & 8.20 & -.\end{array}$

442314103424101 WHITEWOOD CREEK AB SEWAGE TREATMENT PLANT (LAT 4423 14N LONG $1034241 \mathrm{~W}$ )

$\begin{array}{cllllllllllll}\text { MAR } 1986 & 1930 & 12.0 & 622 & 13 & 816 & 795 & 0 & 0 & \ldots & 8.30 & \ldots\end{array}$

06436150 WHITEWOOD CR ABOVE LEAD SD (LAT 4418 O7N LONG 10346 57W)

$\begin{array}{clllllllllll}\text { MAR } 1986 & 1000 & 3.0 & 615 & 2.6 & 355 & 381 & 12.4 & 115 & \ldots & 8.50 & \ldots\end{array}$

442320103422301 WHITEWOOD CREEK BELOW SEWAGE TREATMENT PLANT (LAT 4423 20N LONG 10342 23W) MAR 1986
$13 . .$.
1630
$\begin{array}{lll}7.0 & 624 & 20\end{array}$
786
775
11.9
121
8.10 
Table 38.--Chemical analyses of surface-water samples collected at $14 \mathrm{miscel}$ laneous sites--Continued

\begin{tabular}{|c|c|c|c|c|c|c|c|c|c|c|}
\hline ATE & $\begin{array}{l}\text { NITRO- } \\
\text { GEN,AM- } \\
\text { MONIA + } \\
\text { ORGANIC } \\
\text { DIS. } \\
\text { (MG/L } \\
\text { AS N) } \\
(00623)\end{array}$ & $\begin{array}{c}\text { NITRO- } \\
\text { GEN, } \\
\text { NO2+NO3 } \\
\text { TOTAL } \\
\text { (MG/L } \\
\text { AS N) } \\
(00630)\end{array}$ & $\begin{array}{l}\text { NITRO- } \\
\text { GEN, } \\
\text { NO2+NO3 } \\
\text { OIS- } \\
\text { SOLVED } \\
\text { (MG/L } \\
\text { AS N) } \\
(00631)\end{array}$ & $\begin{array}{c}\text { PHOS- } \\
\text { PHORUS, } \\
\text { TOTAL } \\
\text { (MG/L } \\
\text { AS P) } \\
(00665)\end{array}$ & $\begin{array}{l}\text { PHOS- } \\
\text { PHORUS, } \\
\text { DIS- } \\
\text { SOLVED } \\
\text { (MG/L } \\
\text { AS P) } \\
(00666)\end{array}$ & $\begin{array}{c}\text { CARBON, } \\
\text { ORGANIC } \\
\text { TOTAL } \\
(M G / L \\
\text { AS C) } \\
(00680)\end{array}$ & $\begin{array}{l}\text { CARBON, } \\
\text { ORGANIC } \\
\text { DIS- } \\
\text { SOLVED } \\
\text { (MG/L } \\
\text { AS C) } \\
(00681)\end{array}$ & $\begin{array}{c}\text { CYANIDE } \\
\text { TOTAL } \\
\text { (MG/L } \\
\text { AS CN) } \\
(00720)\end{array}$ & $\begin{array}{l}\text { CYANIDE } \\
\text { DIS- } \\
\text { SOLVED } \\
\text { (MG/L } \\
\text { AS CN) } \\
(00723)\end{array}$ & $\begin{array}{c}\text { SOLIDS, } \\
\text { RESIDUE } \\
\text { AT } 180 \\
\text { DEG. C } \\
\text { DIS- } \\
\text { SOLVED } \\
(M G / L) \\
(70300)\end{array}$ \\
\hline
\end{tabular}

444121101174001 CHEYENME RIVER AT CARLIN FLAT, S.D. (LAT 4441 21N LONG $1011740 \mathrm{~W}$ )

\begin{tabular}{|c|c|c|c|c|c|c|c|c|c|c|}
\hline \multicolumn{11}{|l|}{ AUG 1986} \\
\hline $14 \ldots$ & 0.40 & $<0.100$ & $<0.100$ & 0.160 & 0.012 & 9.3 & 5.3 & -- & $<0.01$ & 1780 \\
\hline $\begin{array}{l}21 \ldots \\
26 \ldots\end{array}$ & -- & $<0.100$ & -- & 0.460 & - & 11 & 5.6 & $\cdots$ & -- & -- \\
\hline SEP & & & & & & & & & & \\
\hline $04 \ldots$ & $\cdots$ &.$<0.100$ & -- & 0.113 & $\cdots$ & 6.3 & 4.4 & -- & -. & \\
\hline
\end{tabular}

442134103443501 GOLD RUN BELOW HOMESTAKE DISCHARGE (LAT 4421 34N LONG $1034435 \mathrm{~W}$ )

MAR 1986

$13 . .$.

$\begin{array}{llllllllll}1.5 & \ldots & 22.0 & 0.450 & 0.360 & \ldots & \ldots & 0.590 & 0.54 & 1130\end{array}$

06436760 HORSE CR ABOVE VALE SD (LAT 4439 O8N LONG 10321 59W)

AUG 1986

$13 . .$.

SEP
$26 .$.

$0.50<0.100<0.100$

0.050

0.008

5.7

5.4

$\therefore \quad<0.01$

1550

0.60

0.400

0.430

0.560

$0.056 \quad 17$

4.5

-. $\quad 0.01$

380

442134103441901 WHITEWOOD CREEK ABOVE GOLD RUN (LAT $442134 N$ LONG 10344 19W)

MAR 1986

$13 . .$.

0.30

$0.490 \quad 0.021$

0.005

$-.<0.010<0.01$

256

06436170 WHITEWOOD CREEK AT DEADWOOD (LAT 4422 48N LONG $1034325 \mathrm{~W}$ )

MAR 1986

13...

0.70

6.70

0.089

0.027

0.160

0.14

539

442314103424101 WhIteWOOO CREEK AB SEWAGE TREATMENT PLANT (LAT 4423 14N LONG $1034241 \mathrm{~W}$ ) MAR 1986

$13 . .$.

0.70

6.40

0.137

0.040

0.120

0.11

535

66436150 WHITEWOOO CR ABOVE LEAD SD (LAT 4418 O7N LONG 10346 57W)

MAR 1986

13.

0.40

0.180

0.029

0.016

$<0.010$

$<0.01$

204

442320103422301 WHITEWOOD CREEK BELON SEWAGE TREATMENT PLANT (LAT $442320 \mathrm{~N}$ LONG 10342 23W) MAR 1986

$13 . .$.

6.30

0.360

0.210

0.110

0.10

504 
Table 38. - - Chemical analyses of surface-water samples collected at 14 miscellaneous sites - - cont inued

\begin{tabular}{|c|c|c|c|c|c|c|c|c|c|c|}
\hline DATE & $\begin{array}{l}\text { SOLIDS, } \\
\text { SUM OF } \\
\text { CONSTI- } \\
\text { TUENTS, } \\
\text { DIS- } \\
\text { SOLVED } \\
\text { (MG/L) } \\
(70301)\end{array}$ & $\begin{array}{l}\text { HARD- } \\
\text { NESS } \\
\text { (MG/L } \\
\text { AS } \\
\text { CACO3) } \\
(00900)\end{array}$ & $\begin{array}{l}\text { HARD- } \\
\text { NESS } \\
\text { NONCAR- } \\
\text { BONATE } \\
\text { (MG/L } \\
\text { AS } \\
\text { CACO3) } \\
(95902)\end{array}$ & $\begin{array}{c}\text { ALKA- } \\
\text { LINITY } \\
\text { LAB } \\
\text { (MG/L } \\
\text { AS } \\
\text { CACO3) } \\
(90410)\end{array}$ & $\begin{array}{l}\text { CALCIUM } \\
\text { DIS- } \\
\text { SOLVED } \\
\text { (MG/L } \\
\text { AS CA) } \\
\text { (00915) }\end{array}$ & $\begin{array}{l}\text { MAGNE- } \\
\text { SIUM, } \\
\text { DIS- } \\
\text { SOLVED } \\
\text { (MG/L } \\
\text { AS MG) } \\
\text { (00925) }\end{array}$ & $\begin{array}{l}\text { SODIUM, } \\
\text { DIS- } \\
\text { SOLVED } \\
\text { (MG/L } \\
\text { AS NA) } \\
(00930)\end{array}$ & $\begin{array}{c}\text { SOOIUM } \\
\text { AD- } \\
\text { SORP- } \\
\text { TION } \\
\text { RATIO } \\
(00931)\end{array}$ & $\begin{array}{l}\text { PERCENT } \\
\text { SODIUM } \\
(00932)\end{array}$ & $\begin{array}{l}\text { POTAS- } \\
\text { SIUM, } \\
\text { DIS- } \\
\text { SOLVED } \\
\text { (MG/L } \\
\text { AS K) } \\
(00935)\end{array}$ \\
\hline
\end{tabular}

444121101174001 CHEYENNE RIVER AT CARLIN FLAT, S.D. (LAT 4441 21N LONG 10117 40W)

\begin{tabular}{|c|c|c|c|c|c|c|c|c|c|c|}
\hline $\begin{array}{l}\text { AUG } 1986 \\
14 \ldots\end{array}$ & 1600 & 770 & 650 & 121 & 170 & 83 & 200 & 3 & 36 & 15 \\
\hline $21 \ldots$ & -. & -- & -- & $\cdots$ & -. & -. & - & $\cdots$ & $\cdots$ & $\cdots$ \\
\hline $\operatorname{SEP}_{\text {SEP }}^{26 . .}$ & - & $\cdots$ & -- & -. & $\cdots$ & $\cdots$ & $\cdots$ & $\cdots$ & $\cdots$ & $\cdot$ \\
\hline $04 \ldots$ & $\cdots$ & -. & $\cdots$ & -. & $-\cdot$ & -. & -. & $\cdots$ & $\cdots$ &.- \\
\hline
\end{tabular}

442134103443501 GOLD RUN BELOW HOMESTAKE DISCHARGE (LAT 4421 34N LONG $1034435 \mathrm{~W}$ )

MAR 1986

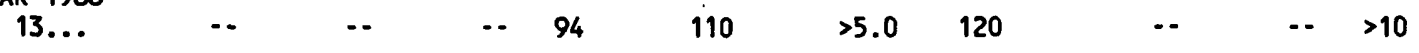

06436760 HORSE CR ABOVE VALE SD (LAT 4439 08N LO 10321 59W)

\begin{tabular}{|c|c|c|c|c|c|c|c|c|c|c|}
\hline $\begin{array}{c}\text { AUG } 1986 \\
13 \ldots\end{array}$ & 1300 & 800 & 640 & 165 & 190 & 80 & 110 & 2 & 23 & 8.2 \\
\hline $\begin{array}{l}\text { SEP } \\
26\end{array}$ & 360 & 170 & 100 & 67 & 44 & 15 & 44 & 2 & 35 & 5.7 \\
\hline
\end{tabular}

442134103441901 WHITEWOOO CREEK ABOVE GOLD RUN (LAT 4421 34N LONG 10344 19W)

$\begin{array}{ccccccccccc}\text { MAR } 1986 & & & & & & & & & & \\ 13 \ldots & \ldots & \ldots & \ldots & 149 & 51 & >5.0 & 7.9 & \ldots & \ldots & 2.0\end{array}$

06436170 WHITEWOOD CREEK AT DEADWOOD (LAT 4422 48N LONG 10343 25W)

MAR 1986

$\begin{array}{llllllllllll}13 . . . & \ldots & \ldots & \ldots & 122 & 71 & >39 & 44 & \ldots & -. & 7.9\end{array}$

442314103424101 WHITEWOOD CREEK AB SEWAGE TREATMENT PLANT (LAT 4423 14N LONG $1034241 \mathrm{~W}$ )

MAR 1986

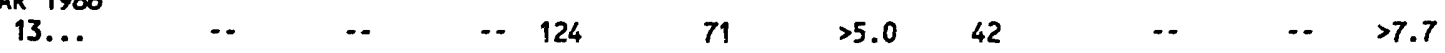

06436150 WHITEWOOD CR ABOVE LEAD SD (LAT 4418 O7N LONG 10346 57W)

MAR 1986

$\begin{array}{lllllllllll}13 . . . & \ldots & \ldots & \ldots & 196 & 45 & >5.0 & 2.4 & \ldots & \ldots & 1.0\end{array}$

442320103422301 WHITEWOOD CREEK BELOW SEWAGE TREATMENT PLANT (LAT $442320 \mathrm{~N}$ LONG 10342 23W) MAR 1986

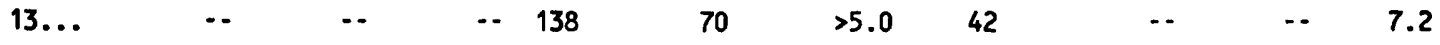


Table 38.-- Chemical analyses of surface-water samples collected at 14 miscellaneous sites--Cont inued

\begin{tabular}{|c|c|c|c|c|c|c|c|c|c|c|}
\hline ITE & $\begin{array}{l}\text { CHLO- } \\
\text { RIDE, } \\
\text { DIS- } \\
\text { SOLVED } \\
\text { (MG/L } \\
\text { AS CL) }\end{array}$ & $\begin{array}{l}\text { SULFATE } \\
\text { DIS- } \\
\text { SOLVED } \\
\text { (MG/L } \\
\text { AS SO4) } \\
(00945)\end{array}$ & $\begin{array}{l}\text { FLUO- } \\
\text { RIDE, } \\
\text { DIS- } \\
\text { SOLVED } \\
\text { (MG/L } \\
\text { AS F) } \\
(00950)\end{array}$ & $\begin{array}{l}\text { SILICA, } \\
\text { DIS- } \\
\text { SOLVED } \\
\text { (MG/L } \\
\text { AS } \\
\text { SIO2) } \\
(00955)\end{array}$ & $\begin{array}{l}\text { ARSENIC } \\
\text { DIS- } \\
\text { SOLVED } \\
\text { (UG/L } \\
\text { AS AS) } \\
(01000)\end{array}$ & $\begin{array}{l}\text { CADMIUM } \\
\text { DIS- } \\
\text { SOLVED } \\
\text { (UG/L } \\
\text { AS CD) } \\
\text { (01025) }\end{array}$ & $\begin{array}{l}\text { COPPER, } \\
\text { DIS- } \\
\text { SOLVED } \\
\text { (UG/L } \\
\text { AS CU) } \\
\text { (01040) }\end{array}$ & $\begin{array}{l}\text { IRON, } \\
\text { DIS- } \\
\text { SOLVED } \\
\text { (UG/L } \\
\text { AS FE) } \\
\text { (01046) }\end{array}$ & $\begin{array}{l}\text { MANGA- } \\
\text { NESE, } \\
\text { DIS- } \\
\text { SOLVED } \\
\text { (UG/L } \\
\text { AS MN) } \\
(01056)\end{array}$ & $\begin{array}{c}\text { MERCURY } \\
\text { DIS- } \\
\text { SOLVED } \\
\text { (UG/L } \\
\text { AS HG) } \\
(71890)\end{array}$ \\
\hline
\end{tabular}

444121101174001 CHEYENNE RIVER AT CARLIN FLAT, S.D. (LAT $444121 \mathrm{~N}$ LONG 10117 40W)

\begin{tabular}{|c|c|c|c|c|c|c|c|c|c|c|}
\hline $\begin{array}{c}\text { AUG } 1986 \\
14 \ldots\end{array}$ & 42 & 970 & 0.60 & 5.0 & 3 & $<1$ & 3 & 20 & $<10$ & $<0.1$ \\
\hline $21 \ldots$ & -. & .. & -. & -. & .. & -. & $\ldots$ & - & -. & -. \\
\hline $26 \ldots$ & -- & -- & $\cdots$ & 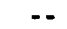 & - & -- & -. & -- & -- & -- \\
\hline SEP & & & & & & & & & & \\
\hline $04 \ldots$ & -. & $\cdots$ & - & $\cdots$ & $\cdots$ & $\cdots$ & -- & -- & $\cdots$ & -- \\
\hline
\end{tabular}

442134103443501 GOLD RUN BELOW HOMESTAKE DISCHARGE (LAT 4421 34N LONG $1034435 \mathrm{~W}$ )

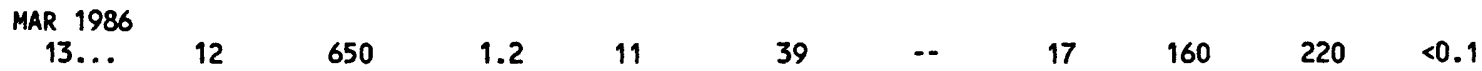

06436760 HORSE CR ABOVE VALE SD (LAT 4439 O8N LONG 10321 59W)

\begin{tabular}{|c|c|c|c|c|c|c|c|c|c|c|}
\hline $\begin{array}{c}\text { AUG } 1986 \\
13 \ldots \\
\text { SEP }\end{array}$ & 14 & 840 & 0.50 & 5.9 & 1 & $<1$ & 2 & 6 & 21 & $<0.1$ \\
\hline $26 \ldots$ & 7.9 & 200 & 0.30 & 5.4 & 3 & $<1$ & 4 & 28 & 8 & $<0.1$ \\
\hline
\end{tabular}

442134103441901 WHITEWOOD CREEK ABOVE GOLD RUN (LAT $442134 \mathrm{~N}$ LONG 10344 19W)

$\begin{array}{cccccccccc}\text { MAR } 1986 & & & & & & & & & \\ 13 \ldots & 12 & 60 & 0.40 & 10 & 3 & \ldots & 2 & 13 & 39\end{array}$

06436170 WHITEWOOD CREEK AT DEADWOOO (LAT 4422 48N LONG 10343 25W)

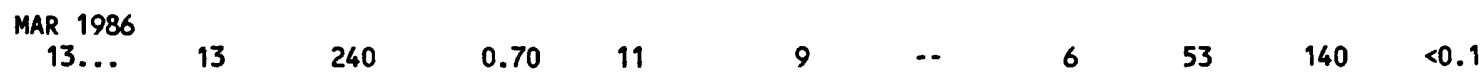

442314103424101 WHITEWOOO CREEK AB SEWAGE TREATMENT PLANT (LAT 4423 14N LONG $1034241 \mathrm{~W}$ )

$\begin{array}{cccccccccc}\text { MAR } 1986 & & & & & & & & & \\ 13 \ldots & 13 & 240 & 0.70 & 11 & 10 & \ldots & 9 & 30 & 130\end{array}$

06436150 WHITEWOOD CR ABOVE LEAD SD (LAT 4418 O7N LONG 10346 57W)
MAR 1986
$13 . .$.
$2.3 \quad 9.9$
$0.10 \quad 10$
$<1 \quad 36$
$6<0.1$

442320103422301 WHITEWOOD CREEK BELOW SEWAGE TREATMENT PLANT (LAT $442320 \mathrm{~N}$ LONG 10342 23W) MAR 1986
$13 . .$.
$19 \quad 210$
$0.70 \quad 11$
14
8
$37 \quad 120$
$<0.1$ 
Table 38.--Chemical analyses of surface-water samples collected at $14 \mathrm{miscellaneous} \mathrm{sites--Continued}$

\begin{tabular}{|c|c|c|c|c|c|c|c|c|c|c|}
\hline ATE & $\begin{array}{l}\text { MERCURY } \\
\text { TOTAL } \\
\text { RECOV- } \\
\text { ERABLE } \\
\text { (UG/L } \\
\text { AS HG) } \\
\text { (71900) }\end{array}$ & $\begin{array}{l}\text { ZINC, } \\
\text { DIS- } \\
\text { SOLVED } \\
\text { (UG/L } \\
\text { AS ZN) } \\
(01090)\end{array}$ & $\begin{array}{l}\text { ANTI- } \\
\text { MONY, } \\
\text { DIS- } \\
\text { SOLVED } \\
\text { (UG/L } \\
\text { AS SB) } \\
(01095)\end{array}$ & $\begin{array}{l}\text { SELE- } \\
\text { NIUM, } \\
\text { DIS- } \\
\text { SOLVED } \\
\text { (UG/L } \\
\text { AS SE) } \\
\text { (01145) }\end{array}$ & $\begin{array}{l}\text { SEDI- } \\
\text { MENT, } \\
\text { SUS- } \\
\text { PENDED } \\
(M G / L) \\
(80154)\end{array}$ & $\begin{array}{l}\text { SED. } \\
\text { SUSP. } \\
\text { SIEVE } \\
\text { DIAM. } \\
\text { \% FINER } \\
\text { THAN } \\
.062 \text { MM } \\
\text { (70331) }\end{array}$ & $\begin{array}{l}\text { SED. } \\
\text { SUSP. } \\
\text { FALL } \\
\text { DIAM. } \\
\text { \% FINER } \\
\text { THAN } \\
.002 \text { MM } \\
\text { (70337) }\end{array}$ & $\begin{array}{l}\text { SED. } \\
\text { SUSP. } \\
\text { FALL } \\
\text { DIAM. } \\
\% \text { FINER } \\
\text { THAN } \\
.004 \text { MM } \\
(70338)\end{array}$ & $\begin{array}{l}\text { SED. } \\
\text { SUSP. } \\
\text { FALL } \\
\text { DIAM. } \\
\text { \% FINER } \\
\text { THAN } \\
\text {.008 MM } \\
\text { (70339) }\end{array}$ & $\begin{array}{l}\text { SED. } \\
\text { SUSP. } \\
\text { FALL } \\
\text { DIAM. } \\
\% \text { FINER } \\
\text { THAN } \\
.016 \text { MM } \\
(70340)\end{array}$ \\
\hline
\end{tabular}

444121101174001 CHEYENNE RIVER AT CARLIN FLAT, S.D. (LAT $444121 \mathrm{~N}$ LONG $1011740 \mathrm{~W}$ )

\begin{tabular}{|c|c|c|c|c|c|c|c|c|c|c|}
\hline $\begin{array}{c}\text { AUG } 1986 \\
14 \ldots\end{array}$ & $\cdots$ & $<10$ & 1 & $\cdots$ & 652 & 99 & 65 & 79 & 90 & 97 \\
\hline $21 \ldots$ & $\ldots$ & -. & -- & $\cdots$ & 241 & 99 & -- & -- & $\ldots$ & -. \\
\hline $26 .$. & $\cdots$ & $\cdots$ & $\cdots$ & $\cdots$ & 77 & 99 & - & - & - & $\cdots$ \\
\hline SEP & & & & & & & & & & \\
\hline $04 \ldots$ & -- & -- & $\cdots$ & $\cdots$ & 128 & 91 & $\cdots$ & -- & -- & - \\
\hline
\end{tabular}

442134103443501 GOLD RUN BELOW HOMESTAKE DISCHARGE (LAT 4421 34N LONG 10344 35W)

MAR 1986

$13 . .$.

63

$7 \quad 17$

61

06436760 HORSE CR ABOVE VALE SD (LAT 4439 08N LONG 10321 59W)

\begin{tabular}{|c|c|c|c|c|c|c|c|c|c|c|}
\hline $\begin{array}{c}\text { AUG } 1986 \\
13 \ldots \\
\text { SEP }\end{array}$ & $-\cdot$ & 10 & $<1$ & $\cdots$ & 25 & 98 & $\cdots$ & - & -- & -- \\
\hline $26 \ldots$ &.- & 3 & $<1$ & -- & 2720 & 97 & 70 & 81 & 88 & 92 \\
\hline
\end{tabular}

442134103441901 WHITEWOOO CREEK ABOVE GOLD RUN (LAT 4421 34N LONG 10344 19W)

MAR 1986

$13 . .$.

0.20

$3<1$

2

06436170 WHITEWOOO CREEK AT DEADWOOD (LAT 4422 48N LONG 10343 25W)

MAR 1986

$13 . .$.

$<0.10<3 \quad 1 \quad 3 \quad 16 \quad 62$

442314103424101 WHITEWOOO CREEK AB SEWAGE TREATMENT PLANT (LAT 4423 14N LONG 10342 41W) MAR 1986

$13 . .$.

$<0.10$

$<3$

$<14$

92

06436150 WHITEWOOO CR ABOVE LEAD SD (LAT 4418 O7N LONG 10346 57W)

MAR 1986

$13 . .$.

$\begin{array}{llllll}0.20 & <3 & < & 1 & 13 & 96\end{array}$

442320103422301 WHITEWOOD CREEK BELOW SEWAGE TREATMENT PLANT (LAT 4423 20N LONG 10342 23W) MAR 1986

$13 . .$.

0.10

$<3$

1

3

14

82 
Table 38.--Chemical analyses of surface-water samples collected at 14 miscellaneous sites--Cont inued

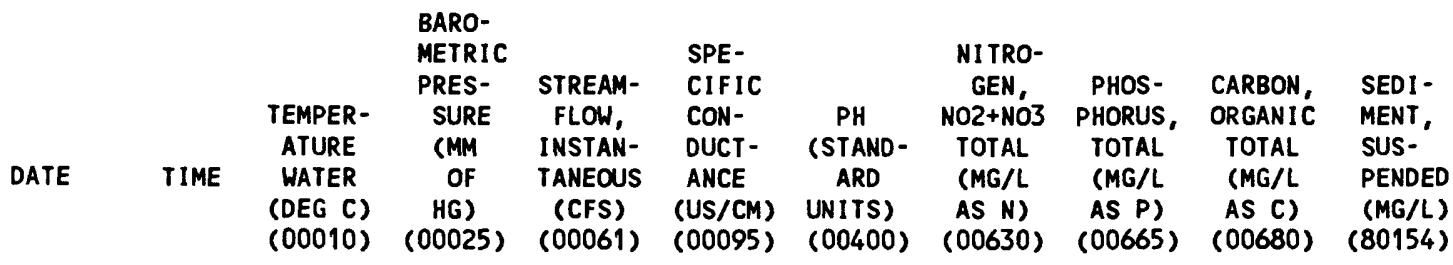

06438000 BELLE FOURCHE RIVER NEAR ELM SPRINGS (LAT $442211 \mathrm{~N}$ LONG 10233 56W)

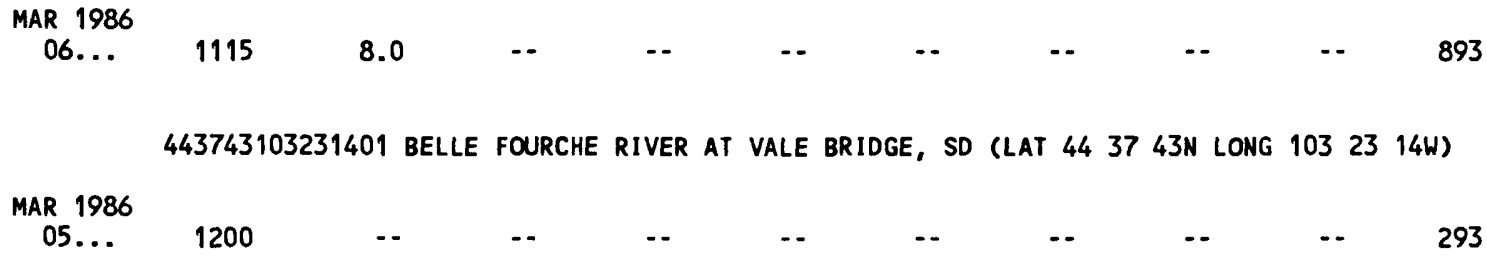

06423500 CHEYENNE RIVER NEAR WASTA,SD (LAT 4404 52N LONG 10224 03W)

JUN 1986

$10 .$.

1745

$16.0 \quad 702$

695

8.60

1.10

23.0

60

39200 
Table 38.--Chemical analyses of surface-water samples collected at 14 miscellaneous sites--Cont inued

$\begin{array}{cllllllll}\text { SED. } & \text { SED. } & \text { SED. } & \text { SED. } & \text { SED. } & \text { SED. } & \text { SED. } & \text { SED. } & \text { SED. } \\ \text { SUSP. } & \text { SUSP. } & \text { SUSP. } & \text { SUSP. } & \text { SUSP. } & \text { SUSP. } & \text { SUSP. } & \text { SUSP. } & \text { SUSP. } \\ \text { SIEVE } & \text { FALL } & \text { FALL } & \text { FALL } & \text { FALL } & \text { FALL } & \text { FALL } & \text { FALL } & \text { FALL } \\ \text { DIAM. } & \text { DIAM. } & \text { DIAM. } & \text { DIAM. } & \text { DIAM. } & \text { DIAM. } & \text { DIAM. } & \text { DIAM. } & \text { DIAM. } \\ \% \text { FINER } & \% \text { FINER } & \% & \text { FINER } & \% \text { FINER } & \% \text { FINER } & \% \text { FINER } & \% \text { FINER } & \% \text { FINER } \% \text { FINER } \\ \text { THAN } & \text { THAN } & \text { THAN } & \text { THAN } & \text { THAN } & \text { THAN } & \text { THAN } & \text { THAN } & \text { THAN } \\ .062 M M & .002 M M & .004 M M & .008 M M & .016 M M & .062 M M & 125 M M & .250 M M & .500 M M \\ (70331) & (70337) & (70338) & (70339) & (70340) & (70342) & (70343) & (70344) & (70345)\end{array}$

06438000 BELLE FOURCHE RIVER NEAR ELM SPRINGS (LAT $442211 \mathrm{~N}$ LONG 10233 56W)

MAR 1986

$\begin{array}{llllllllll}06 . . . & 100 & 59 & 70 & 80 & 87 & 97 & 99 & 100 & \ldots\end{array}$

443743103231401 BELLE FOURCHE RIVER AT VALE BRIDGE, SD (LAT 4437 43N LONG 10323 14W)

MAR 1986

$05 . .$.

99

06436100 BELLE FOURCHE RIVER BELOW NISLAND, SD (LAT 4440 12N LONG $1032930 \mathrm{~W}$ )

JUL 1986

08...

99

443738103152201 BELLE FOURCHE RIVER BELOW VALE, S.D. (LAT 4437 38N LONG 10315 22W)

MAR 1986

$05 . .$.

59

69

79

87

$99 \quad 100$

442252103571801 BELLE FOURCHE RIVER NEAR HEREFORD, S.D. (LAT 4422 52N LONG 10357 18W)

MAR 1986

06...

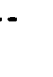

66

75

83

95

$97 \quad 100$

06423500 CHEYENNE RIVER NEAR WASTA,SD (LAT 4404 52N LONG 10224 03W)

JUN 1986

$10 . .$.

61

70

78

85

98

99

99

99 
Table 39.--Total chemical analysis of suspended sediment

[Iron, manganese, aluminum, and titanium in weight percent; $\mu \mathrm{g} / \mathrm{g}$, micrograms per gram; $\mu \mathrm{m}$, micrometers; $\mathrm{ft}^{3} / \mathrm{s}$, cublc feet per second; $\mathrm{mg} / \mathrm{L}$, milligrams per liter; $<$, less than; >, greater than; --, not analyzed]

\begin{tabular}{|c|c|c|c|c|c|c|c|c|}
\hline $\begin{array}{l}\text { Site } \\
\text { number in } \\
\text { figures } 1 \\
\text { and } 2\end{array}$ & Date & Time & $\begin{array}{c}\text { Size } \\
\text { fraction } \\
(\mu \mathrm{m})\end{array}$ & $\begin{array}{l}\text { Dis- } \\
\text { charge } \\
\left(\mathrm{ft}^{3} / \mathrm{s}\right)\end{array}$ & $\begin{array}{l}\text { Sediment } \\
\text { concen- } \\
\text { tration } \\
(\mathrm{mg} / \mathrm{L})\end{array}$ & Iron & $\begin{array}{c}\text { Manga- } \\
\text { nese }\end{array}$ & $\begin{array}{l}\text { Alumi- Titan- } \\
\text { num ium }\end{array}$ \\
\hline
\end{tabular}

Whi tewood Creek above Whitewood

$\begin{array}{rrrrrrrrrr}10 & 04-23-86 & 1330 & & 165 & 213 & 8.0 & 0.26 & 5.6 & 0.32 \\ 10 & 04-23-86 & 1330 & & 165 & 213 & -- & -- & -- & - \\ 10 & 06-09-86 & 1955 & & 89 & 728 & 7.7 & .20 & 5.6 & .27 \\ 10 & 06-10-86 & 0030 & & 75 & 466 & 7.4 & .20 & 7.0 & .34 \\ 10 & 06-10-86 & 0030 & <4 & 75 & 466 & 7.6 & .20 & 7.8 & .32 \\ 10 & 06-10-86 & 0030 & 4-16 & 75 & 466 & 7.2 & .20 & 6.8 & .26 \\ 10 & 06-10-86 & 0030 & 16-62 & 75 & 466 & 7.8 & .20 & 7.6 & .28 \\ 10 & 06-10-86 & 0030 & 16-62 & 75 & 466 & -- & -- & -- & - \\ 10 & 06-10-86 & 1515 & & 146 & 247 & 7.7 & .21 & 6.2 & .26\end{array}$

Whitewood Creek above Vale

\begin{tabular}{|c|c|c|c|c|c|c|c|c|c|}
\hline 15 & $02-26-86$ & 1130 & & 92 & 1,160 & 6.1 & .20 & 5.5 & .29 \\
\hline 15 & $02-26-86$ & 1130 & & 92 & 1,160 & 5.7 & .16 & 6.2 & .26 \\
\hline 15 & $04-24-86$ & 1445 & & 145 & 380 & 6.7 & .17 & 5.8 & .25 \\
\hline 15 & $06-10-86$ & 1345 & & 158 & 4,480 & 6.7 & .17 & 6.2 & .28 \\
\hline 15 & $06-10-86$ & 1345 & $<4$ & 158 & 4,480 & 6.7 & .18 & 6.6 & .28 \\
\hline 15 & $06-10-86$ & 1345 & $4-16$ & 158 & 4,480 & 6.4 & .17 & 6.0 & .24 \\
\hline 15 & $06-10-86$ & 1345 & $16-62$ & 158 & 4,480 & 6.4 & .17 & 5.8 & .25 \\
\hline 15 & $06-10-86$ & 1345 & $>62$ & 158 & 4,480 & 6.4 & .16 & 2.4 & .12 \\
\hline
\end{tabular}

Belle Fourche River near Sturgis

$\begin{array}{lrrrrrrrrr}26 & 03-03-86 & 1400 & & 1,470 & 3,960 & 4.7 & .09 & 8.0 & .36 \\ 26 & 03-05-86 & 1015 & & 1,580 & 4,070 & 4.3 & .06 & 7.8 & .37 \\ 26 & 03-21-86 & 1205 & & 236 & 340 & 4.8 & .08 & 9.2 & .42 \\ 26 & 04-09-86 & 1230 & & 338 & 2,070 & 4.6 & .04 & 1.0 & .35 \\ 26 & 04-09-86 & 1230 & <4 & 338 & 2,070 & 4.2 & .04 & 8.6 & .44 \\ 26 & 04-09-86 & 1230 & 4-16 & 338 & 2,070 & 4.2 & .05 & 8.7 & .39 \\ 26 & 04-09-86 & 1230 & 16-62 & 338 & 2,070 & 4.4 & .05 & 9.1 & .39 \\ 26 & 05-01-86 & 1425 & & 501 & 870 & 4.4 & .06 & 8.9 & .40 \\ 26 & 09-25-86 & 1254 & & 5,360 & 6,970 & 4.2 & .08 & 7.9 & .38\end{array}$




\begin{tabular}{|c|c|c|c|c|c|c|c|c|c|c|c|}
\hline $\begin{array}{c}\text { Arse- } \\
\text { nic } \\
(\mu g / g)\end{array}$ & $\begin{array}{c}\text { Anti- } \\
\text { money } \\
(\mu \mathrm{g} / \mathrm{g})\end{array}$ & $\begin{array}{l}\text { Cad- } \\
\text { mium } \\
(\mu g / g)\end{array}$ & $\begin{array}{l}\text { Chrom- } \\
\text { ium } \\
\text { ( } \mu \mathrm{g} / \mathrm{g})\end{array}$ & $\begin{array}{l}\text { Co- } \\
\text { balt } \\
(\mu g / g)\end{array}$ & $\begin{array}{l}\text { Cop- } \\
\text { per } \\
(\mu \mathrm{g} / \mathrm{g})\end{array}$ & $\begin{array}{l}\text { Lead } \\
(\mu \mathrm{g} / \mathrm{g})\end{array}$ & $\begin{array}{l}\text { Mer- } \\
\text { cury } \\
(\mu \mathrm{g} / \mathrm{g})\end{array}$ & $\begin{array}{l}\text { Nickel } \\
(\mu \mathrm{g} / \mathrm{g})\end{array}$ & $\begin{array}{l}\text { Sele- } \\
\text { nium } \\
(\mu \mathrm{g} / \mathrm{g})\end{array}$ & $\begin{array}{c}\text { Zinc } \\
(\mu g / g)\end{array}$ & $\begin{array}{l}\text { Total } \\
\text { organic } \\
\text { carbon } \\
(\mu g / g)\end{array}$ \\
\hline
\end{tabular}

$\begin{array}{rrrrrrrrrrrr}880 & 16.2 & 1.6 & -- & 15 & 276 & 116 & 2.22 & 49 & 3.4 & 285 & 3.8 \\ -- & -- & -- & -- & -- & -- & -- & -- & -- & -- & -- & 4.4 \\ 860 & 21.0 & 1.0 & -- & 26 & 221 & 131 & 2.14 & 87 & 2.7 & 555 & 5.1 \\ 640 & 18.2 & 1.8 & -- & 26 & 182 & 134 & 2.10 & 74 & 2.4 & 460 & 4.4 \\ 640 & 18.6 & 1.2 & -- & 22 & 370 & 132 & 2.11 & 98 & 2.2 & 570 & 4.5 \\ 600 & 17.6 & 1.2 & -- & 20 & 496 & 136 & 2.26 & 128 & 2.2 & 650 & 4.5 \\ 640 & 18.2 & <.5 & -- & 20 & 222 & 138 & 2.06 & 88 & 3.0 & 480 & 4.4 \\ -- & -- & -- & -- & -- & -- & -- & -- & -- & -- & -- & 4.5 \\ 1,300 & 29.0 & <.5 & -- & 22 & 185 & 116 & 2.36 & 74 & 2.3 & 475 & 4.1\end{array}$

$\begin{array}{rrrrrrrrrrrr}990 & 2.0 & <.5 & 54 & 74 & 54 & 23 & .08 & 23 & 2.0 & 116 & -- \\ 840 & 2.6 & <.5 & -- & 12 & 61 & 20 & .94 & 36 & 1.9 & 110 & 2.0 \\ 1,900 & 4.8 & <.5 & -- & 17 & 114 & 42 & 1.16 & 52 & 2.3 & 205 & 2.3 \\ 2,100 & 8.3 & <.5 & -- & 16 & 129 & 59 & 1.49 & 54 & 2.4 & 260 & 3.2 \\ 1,000 & 9.1 & <.5 & -- & 17 & 262 & 76 & 1.78 & 68 & 2.5 & 380 & 3.5 \\ 960 & 7.7 & <.5 & -- & 17 & 177 & 56 & 1.53 & 58 & 2.3 & 280 & 3.3 \\ 1,000 & 7.2 & <.5 & -- & 15 & 120 & 51 & 1.32 & 48 & 2.1 & 235 & 3.1 \\ 1,200 & 2.8 & <.5 & -- & 10 & 46 & 2 & .45 & 20 & 1.4 & 70 & 1.7\end{array}$

$\begin{array}{rrrrrrrrrrrr}210 & 1.2 & <.5 & -- & 12 & 40 & 13 & .27 & 35 & 1.4 & 115 & 1.1 \\ 124 & 1.2 & <.5 & -- & 13 & 38 & 13 & .25 & 38 & 1.1 & 124 & \vdots .1 \\ 116 & 1.2 & <.5 & -- & 8 & 40 & 14 & .34 & 48 & 1.2 & 152 & 1.6 \\ 34 & 1.0 & <.5 & 99 & 14 & 30 & 15 & .12 & 43 & 1.0 & 147 & - \\ 32 & 1.2 & 7.2 & -- & 14 & 1,080 & 72 & .86 & 146 & .8 & 770 & 1.1 \\ 36 & 1.3 & 4.8 & -- & 14 & 435 & 41 & .76 & 90 & .9 & 370 & 1.3 \\ 30 & 1.3 & <.5 & -- & 15 & 96 & 22 & .27 & 50 & .9 & 175 & 1.2 \\ 52 & 1.3 & <.5 & -- & 13 & 39 & 15 & .20 & 46 & 1.0 & 148 & 1.0 \\ 188 & 1.3 & <.5 & -- & 11 & 40 & 17 & .36 & 37 & 1.6 & 126 & 1.3\end{array}$


Table 39.--Total chemical analysis of suspended sediment--Continued

\begin{tabular}{|c|c|c|c|c|c|c|c|c|c|}
\hline $\begin{array}{l}\text { Site } \\
\text { number in } \\
\text { figures } 1 \\
\text { and } 2\end{array}$ & Date & Time & $\begin{array}{c}\text { Size } \\
\text { fraction } \\
(\mu \mathrm{m})\end{array}$ & $\begin{array}{l}\text { Dis- } \\
\text { charge } \\
\left(\mathrm{ft}^{3} / \mathrm{s}\right)\end{array}$ & $\begin{array}{l}\text { Sediment } \\
\text { concen- } \\
\text { tration } \\
(\mathrm{mg} / \mathrm{L})\end{array}$ & Iron & $\begin{array}{c}\text { Manga- } \\
\text { nese }\end{array}$ & $\begin{array}{l}\text { Alumi- } \\
\text { num }\end{array}$ & $\begin{array}{l}\text { Titan- } \\
\text { ium }\end{array}$ \\
\hline
\end{tabular}

Cheyenne River at Cherry Creek

\begin{tabular}{|c|c|c|c|c|c|c|c|c|c|}
\hline 37 & $04-22-86$ & 1245 & & 4,890 & 6,030 & 4.1 & 0.04 & 8.3 & .36 \\
\hline 37 & $04-29-86$ & 1230 & & 8,120 & 8,040 & 4.2 & .05 & 8.2 & -35 \\
\hline 37 & $04-29-86$ & 1230 & $<4$ & 8,120 & 8,040 & 3.8 & .07 & 7.8 & .40 \\
\hline 37 & $04-29-86$ & 1230 & $4-16$ & 8,120 & 8,040 & 4.0 & .70 & 7.9 & .38 \\
\hline 37 & $04-29-86$ & 1230 & $4-16$ & 8,120 & 8,040 & -- & -- & -- & \\
\hline 37 & $04-29-86$ & 1230 & $16-62$ & 8,120 & 8,040 & 4.2 & .06 & 8.6 & $\cdot 3$ \\
\hline 37 & $04-29-86$ & 1230 & $16-62$ & 8,120 & 8,040 & -- & -- & -- & \\
\hline 37 & $04-29-86$ & 1230 & $>62$ & 8,120 & 8,040 & 2.1 & .07 & 3.1 & .14 \\
\hline 37 & $05-07-86$ & 1330 & & 428 & 429 & 4.4 & .07 & 8.3 & .34 \\
\hline 37 & $05-14-86$ & 1130 & & 5,240 & 6,870 & 4.1 & .05 & 8.2 & \\
\hline 37 & $05-29-86$ & 1240 & & 565 & 199 & 3.8 & .1 & 7.6 & \\
\hline 37 & $05-29-86$ & 1240 & & 565 & 199 & 3.5 & .10 & 7.0 & . \\
\hline 37 & $06-11-86$ & 1500 & & 12,900 & 27,600 & 3.7 & .05 & 7.9 & \\
\hline 37 & $06-26-86$ & 1310 & & 977 & 743 & 3.8 & .07 & 7.3 & .2 \\
\hline 37 & $07-10-86$ & 1340 & & 565 & 517 & 4.1 & .03 & 8.0 & \\
\hline 37 & $07-24-86$ & 1230 & & 525 & 201 & 3.3 & .05 & 6.8 & \\
\hline 37 & $08-07-86$ & 1530 & & 352 & 160 & 3.8 & .05 & 7.4 & \\
\hline 37 & $08-07-86$ & 1530 & & 352 & 160 & 3.6 & .07 & 7.7 & \\
\hline 37 & $08-07-86$ & 1530 & & 352 & 160 & -- & -- & -- & \\
\hline 37 & $08-14-86$ & 1655 & & 432 & 432 & 4.1 & .04 & 8.2 & .3 \\
\hline 37 & $08-21-86$ & 1235 & & 352 & 159 & 3.6 & .09 & 7.4 & $\bullet$ \\
\hline 37 & $08-26-86$ & 1440 & & 339 & 71 & 3.5 & .13 & 7.4 & .3 \\
\hline 37 & $09-04-86$ & 1545 & & 475 & 504 & 4.0 & .05 & 7.7 & \\
\hline 37 & $09-27-86$ & 1405 & & 8,700 & 17,100 & 3.5 & .10 & 7.0 & \\
\hline 37 & $09-27-86$ & 1405 & & 8,700 & 17,100 & 4.2 & .08 & 8.0 & \\
\hline 37 & $09-27-86$ & 1405 & & 8,700 & 17,100 & -- & -- & -- & \\
\hline
\end{tabular}

Miscellaneous sites; names listed in table 1

\begin{tabular}{lrrrrrrrr}
23 & $08-13-86$ & 1230 & 61 & 25 & 3.5 & .15 & 7.0 & .50 \\
23 & $09-26-86$ & 1630 & 2,390 & 241 & 4.5 & .04 & 9.6 & .42 \\
24 & $03-05-86$ & 1130 & -- & 3,660 & 4.2 & .05 & 8.5 & .39 \\
24 & $03-05-86$ & 1130 & -- & 3,660 & 4.4 & .05 & 9.4 & .41 \\
28 & $03-06-86$ & 1245 & -- & 6,230 & 4.7 & .09 & 8.4 & .39 \\
30 & $03-06-86$ & 1115 & 5,133 & 10,600 & 5.0 & .08 & 8.35 & .34 \\
32 & $06-10-86$ & 1745 & 8,100 & 38,300 & 3.65 & .05 & 7.2 & .32 \\
38 & $08-14-86$ & 1425 & 503 & 652 & 4.0 & .04 & 8.0 & .38 \\
38 & $08-21-86$ & 1230 & 441 & 241 & 3.6 & .07 & 7.4 & .33 \\
38 & $08-26-86$ & 1700 & 332 & 77 & 3.5 & .12 & 7.2 & .33 \\
38 & $09-04-86$ & 1257 & 494 & 127 & 3.8 & .07 & 7.0 & .33 \\
\hline
\end{tabular}




\begin{tabular}{|c|c|c|c|c|c|c|c|c|c|c|c|}
\hline $\begin{array}{c}\text { Arse- } \\
\text { nic } \\
(\mu \mathrm{g} / \mathrm{g})\end{array}$ & $\begin{array}{c}\text { Anti- } \\
\text { money } \\
(\mu \mathrm{g} / \mathrm{g})\end{array}$ & $\begin{array}{l}\text { Cad- } \\
\text { mium } \\
(\mu \mathrm{g} / \mathrm{g})\end{array}$ & $\begin{array}{l}\text { Chrom- } \\
\text { lum } \\
(\mu \mathrm{g} / \mathrm{g})\end{array}$ & $\begin{array}{l}\text { Co- } \\
\text { balt } \\
(\mu g / g)\end{array}$ & $\begin{array}{l}\text { Cop- } \\
\text { per } \\
(\mu \mathrm{g} / \mathrm{g})\end{array}$ & $\begin{array}{l}\text { Lead } \\
(\mu \mathrm{g} / \mathrm{g})\end{array}$ & $\begin{array}{l}\text { Mer- } \\
\text { cury } \\
(\mu \mathrm{g} / \mathrm{g})\end{array}$ & $\begin{array}{l}\text { Nickel } \\
(\mu \mathrm{g} / \mathrm{g})\end{array}$ & $\begin{array}{l}\text { Sele- } \\
\text { nium } \\
(\mu \mathrm{g} / \mathrm{g})\end{array}$ & $\begin{array}{l}\text { Zinc } \\
(\mu \mathrm{g} / \mathrm{g})\end{array}$ & $\begin{array}{l}\text { Total } \\
\text { organic } \\
\text { carbon } \\
(\mu g / g)\end{array}$ \\
\hline 22 & 1.2 & $<.5$ & 76 & 10 & 29 & 18 & 0.11 & 40 & 1.2 & 125 & -- \\
\hline 36 & 1.1 & $<.5$ & 71 & 11 & 26 & 19 & .08 & 35 & 1.0 & 129 & -- \\
\hline 44 & 1.2 & 1.1 & -- & 14 & 750 & 53 & .33 & 66 & .9 & 510 & $<.1$ \\
\hline 50 & 1.3 & 2.5 & -- & 12 & 471 & 113 & .68 & 64 & .9 & 350 & $<.1$ \\
\hline-- & -- & -- & -- & -- & -- & -- & -- & -- & -- & -- & $<.1$ \\
\hline 36 & 1.1 & $<.5$ & -- & 12 & 78 & 20 & .18 & 38 & .7 & 147 & 1.5 \\
\hline-- & -- & -- & -- & -- & -- & -- & .19 & -- & -- & -- & 1.4 \\
\hline 50 & 1.2 & $<.5$ & -- & 5 & - & 12 & .04 & 23 & .6 & 55 & $<.1$ \\
\hline 36 & 1.1 & $<.5$ & 78 & 13 & 31 & 20 & .08 & 38 & 1.2 & 170 & -- \\
\hline 24 & 1.3 & $<.5$ & 72 & 11 & 29 & 22 & .09 & 40 & 1.4 & 171 & -- \\
\hline 32 & 1.1 & $<.5$ & 63 & 7 & 28 & 13 & .09 & 32 & 1.6 & 118 & -- \\
\hline 30 & 1.0 & $<.5$ & -- & 10 & 20 & 20 & .45 & 35 & 1.0 & 115 & 2.8 \\
\hline 12 & 1.0 & $<.5$ & 40 & 8 & 21 & 21 & .06 & 21 & .04 & 98 & -- \\
\hline 32 & .9 & $<.5$ & 61 & 5 & 24 & 16 & .05 & 25 & 1.2 & 102 & -- \\
\hline 13 & .8 & $<.5$ & 63 & 11 & 26 & 15 & .15 & 35 & .7 & 120 & -- \\
\hline 23 & .7 & $<.5$ & 56 & 7 & 22 & 15 & .08 & 26 & .8 & 98 & -- \\
\hline 21 & .8 & $<.5$ & 80 & 9 & 24 & 13 & .17 & 35 & .9 & 117 & -- \\
\hline 26 & 1.0 & $<.5$ & -- & 11 & 25 & 13 & .18 & 32 & .9 & 114 & 1.9 \\
\hline-- & -- & -- & -- & -- & -- & -- & .18 & -- & -- & -- & -- \\
\hline 15 & .9 & $<.5$ & 93 & 12 & 26 & 14 & .17 & 40 & 1.0 & 117 & -- \\
\hline 22 & .8 & $<.5$ & 54 & 9 & 25 & 18 & .16 & 28 & .8 & 106 & -- \\
\hline 28 & .9 & $<.5$ & 55 & 8 & 24 & 17 & .20 & 28 & .9 & 108 & -- \\
\hline 13 & .9 & $<.5$ & 40 & 10 & 24 & 21 & .06 & 24 & .5 & 113 & -- \\
\hline 30 & 1.0 & $<.5$ & 95 & 10 & 25 & 27 & .45 & 35 & 1.0 & 115 & 2.8 \\
\hline 78 & 1.1 & $<.5$ & -- & 13 & 36 & 16 & .14 & 35 & .9 & 120 & $<.1$ \\
\hline-- & -- & -- & -- & -- & -- & -- & -- & -- & -- & -- & $<.1$ \\
\hline 10 & .5 & 3.0 & -- & 20 & 25 & 30 & .52 & 30 & 1.5 & 115 & 2.8 \\
\hline 14 & 1.1 & $<.5$ & -- & 16 & 34 & 20 & .33 & 45 & .9 & 148 & .8 \\
\hline 50 & 1.1 & $<.5$ & -- & 15 & 32 & 21 & .12 & 36 & 1.1 & 122 & .9 \\
\hline 42 & 1.0 & $<.5$ & -- & 16 & 34 & 20 & .12 & 38 & 1.1 & 128 & .9 \\
\hline 136 & 1.2 & $<.5$ & -- & 15 & 34 & 18 & .16 & 37 & 1.1 & 122 & $<.1$ \\
\hline 140 & 1.0 & $<.5$ & 7.5 & 16 & 32 & 18 & .15 & 37 & 1.0 & 130 & -- \\
\hline 9.2 & 0.9 & $<.5$ & 40 & 9 & 24 & 22 & .04 & 20 & .2 & 95 & -- \\
\hline 19 & 1.2 & $<.5$ & 73 & 9 & 29 & 14 & .11 & 44 & 1.8 & 126 & -- \\
\hline 19 & 0.9 & $<.5$ & 52 & 8 & 23 & 19 & .08 & 28 & .7 & 97 & -- \\
\hline 28 & 0.9 & $<.5$ & 53 & 6 & 23 & 15 & .22 & 32 & 1.0 & 103 & -- \\
\hline 29 & 1.1 & $<.5$ & 65 & 9 & 27 & 18 & .21 & 38 & 1.2 & 116 & -- \\
\hline
\end{tabular}


Samples for organic carbon analyses normally were collected by depth integration at the centroid of the stream. Samples for total organic carbon were collected in small glass bottles. Samples for dissolved organic carbon analyses were collected in a stainless steel container, pressurized with nitrogen gas, and filtered through $0.45-\mu \mathrm{m}$ silver filters into similar small glass bottles. The bottles were then put on ice and shipped to the Central Laboratory for analysis. Organic carbon analysis data are listed in tables 34 through 39.

There were times when normal sampling procedures could not be followed. During some storms, the streams were too deep to wade or the stage was changing too fast to take a 15-vertical sample. During these storms, aliquots were collected from a bridge or cable way at 5 to 8 verticals, or a sample was collected by wading to a point nearest the centroid as practicable. At these times, suspended-sediment samples were collected directly from the stream rather than poured from the churn, and some samples for organic carbon analyses were taken from the churn rather than from the centroid of the stream. A few other times, 5 to 8 vertical samples were collected when the stream was thickly covered with ice and weather conditions were dangerous.

Four field readings--water temperature, dissolved oxygen, pH, and specific conductance--were determined at the site. Most often readings were determined at the centroid of the stream, while at other times averages were computed from readings determined at 3 to 20 verticals across the channel. Several meters were used in the study: a Hydrolab Environmental Data Systems Model 4041 Digital four-parameter meter, an ODEC 4-parameter meter, and an Orion pH meter. The meters were calibrated at each site or at least daily during field trips.

The Hydrolab was the instrument used most often. The Hydrolab instrument consists of a Sonde unit, which contains the sensors, a connecting cable, and an indicator unit. The Sonde consists of a temperature thermistor, a fourelectrode conductivity cell, a glass pH electrode, a Lazaran reference electrode, and a Clark polarographic dissolved oxygen cell. The indicator unit consists of a digital readout, a rotary switch to select the parameter for readout, and calibration controls. The instrument is fully temperature compensated. The ODEC is similar to the Hydrolab in function and was used when the Hydrolab was unavailable. The $\mathrm{pH}$ meters were equipped with $\mathrm{pH}$ and reference electrodes and were used when the Hydrolab's pH readings were slow or suspect. 


\section{REFERENCES}

Barnett, P.R., Mallory, E.C., JR., 1971, Determination of minor elements in water by emission spectroscopy: Techniques of Water-Resources Investigations of the U. S. Geological Survey, Book 5, Chap A2, 31 p.

Buchanan, T.J., and Somers, W.P., 1969, Discharge measurements at gaging stations: Techniques of Water-Resources Investigations of the $U$. $S$. Geological Survey, Book 3, Chap A8, 65 p.

1982, Stage measurement at gaging stations: Techniques of WaterResources Investigations of the U. S. Geological Survey, Book 3, Chap A7, $28 \mathrm{p}$.

Craig, J.D., 1983, Installation and service manual for U.S. Geological Survey manometers: Techniques of Water-Resources Investigations of the U.S. Geological Survey, Book 8, Chap A2, 57 p.

Friedman, L. C., and Erdmann, D. E., 1982, Quality Assurance practices for the chemical and biological analyses of water and fluvial sediments: Techniques of Water-Resources Investigations of the U.S. Geological Survey, Book 3, Chap A8, 65 p.

Guy, H.P., 1969, Laboratory theory and methods for sediment analysis: Techniques of Water-Resources Investigations of the U.S. Geological Survey, Book 5, Chap C1, 58 p.

Guy, H.P., and Norman, V.W., 1982, Field methods for measurement of fluvial sediment: Techniques of Water-Resources Investigations of the U.S. Geological Survey, Book 3, Chap C2, 58 p.

Horowitz, A.J., and Elrick, K.A., 1985, Multielement analysis of rocks and sediments by wet digestion and atomic absorption spectroscopy (unpublished draft).

Kennedy, E.J., 1983, Computation of continuous records of streamflow: Techniques of Water-Resources Investigations of the U.S. Geological Survey, Book 3, Chap A13, 53 p.

--- 1984, Discharge ratings at gaging stations: Techniques of WaterResources Investigations of the U.S. Geological Survey, Book 3, Chap A10, $59 \mathrm{p}$.

Skougstad, M.W., Fishman, M.J., Friedman, L.C., Erdmann, D.E., and Duncan, S.S., eds., 1979, Methods for determination of inorganic substances in water and fluvial sediments: Techniques of Water-Resources Investigations of the U.S. Geological Survey, Book 5, Chap A1, 626 p.

Wershaw, R.L., Fishman, M.J., Grabbe, R.R., and Lowe, L.E., 1987, Methods for the determination of organic substances in water and fluvial sediments: Techniques of Water-Resources Investigations of the U.S. Geological Survey, Book 5, Chap A3, 80 p. 


\title{
Chapter F. Arsenic and Iron Versus Filter Poresize in Whitewood Creek and the Belle Fourche River
}

\author{
By Christopher C. Fuller, James A. Davis, Regina G. Claypool-Frey
}

\section{SAMPLE COLLECTION AND HANDLING PROCEDURES}

In order to determine the dissolved and colloidal components of arsenic and iron in filtrates from the commonly used 0.45-um filtrations, sets of serial filtrations were conducted in August 1986. Samples were collected at five sites on Whitewood Creek and one site on the Belle Fourche River: Whitewood Creek at Deadwood (site 7 , above sewage treatment effluent discharge); Whitewood Creek above Whitewood (site 10); Whitewood Creek at Berger site (site 13) above and below large ground-water seep; Whitewood Creek above Vale (site 15); Whitewood Creek above Sheeler site (site 17); and Belle Fourche River at Vale (site 20) (see figs. 1 and 2, table 1).

At each site, raw water samples were collected mid-stream with a streamwater rinsed 2-L polyethylene wide mouth jar that was filled by submersion with the opening held mid-depth oriented into the flow. Splits were immediately filtered by vacuum through $0.45-$ and $0.1-\mu \mathrm{m}$ pore-size cellulose nitrate membrane filters ( $47 \mathrm{~mm}$, diameter) using polycarbonate filter towers. A fraction of the $0.45-\mu \mathrm{m}$ filtrate was subsequently filtered through a $0.01-\mu \mathrm{m}$ cellulose nitrate filter. An aliquot of the $0.45-\mu \mathrm{m}$ filtrate was transferred to an acid-cleaned polyethylene bottle and stored refrigerated without acidification for major cation and anion analyses. The remaining $0.45 \mu \mathrm{m}$ filtrates, and the $0.1-$ and $0.01-\mu \mathrm{m}$ filtrates were acidified in the collection reservoirs to 1 percent $(\mathrm{v} / \mathrm{v})$ with concentrated $\mathrm{HCL}$, transferred to polyethylene bottles and stored refrigerated. An aliquot of the acidified 0.1-um filtrate was processed immediately for arsenate-arsenite speciation by the anion exchange technique of Ficklin (1983). Stream pH was measured directly in the streamflow with a combination electrode. Total $\mathrm{CO}_{2}$ samples were collected by syringe and injected into septa sealed vials. All sample containers and sampling apparatus were acid-washed before use in the field.

\section{ANALYTICAL TECHNIQUES AND RESULTS}

Major cations were determined by flame atomic absorption. Anions $\left(\mathrm{SO}_{4}{ }^{2}, \mathrm{Cl}^{-}\right.$) were determined by ion chromatography utilizing a conductivity detector and a borate-gluconate buffer for the mobile phase. Total $\mathrm{CO}_{2}$ was determined via gas chromatography of the headspace in the sample vials following acidification (Culbertson and others, 1981). Total iron was determined colorimetrically by the ferrozine method (Stookey and others, 1970), utilizing $10 \mathrm{~cm}$ pathlength spectrophotometer cells. Total arsenic, and the separated arsenate and arsenite fractions were determined by graphite furnace atomic absorption using operating conditions similar to Ficklin's (1983).

Analytical results are presented in table 40. Arsenate accounted for greater than 90 percent of the total arsenic; therefore, only total arsenic concentrations are presented. Total iron and arsenic concentrations versus filter poresize are listed in table 41 and presented graphically in figure 4. 


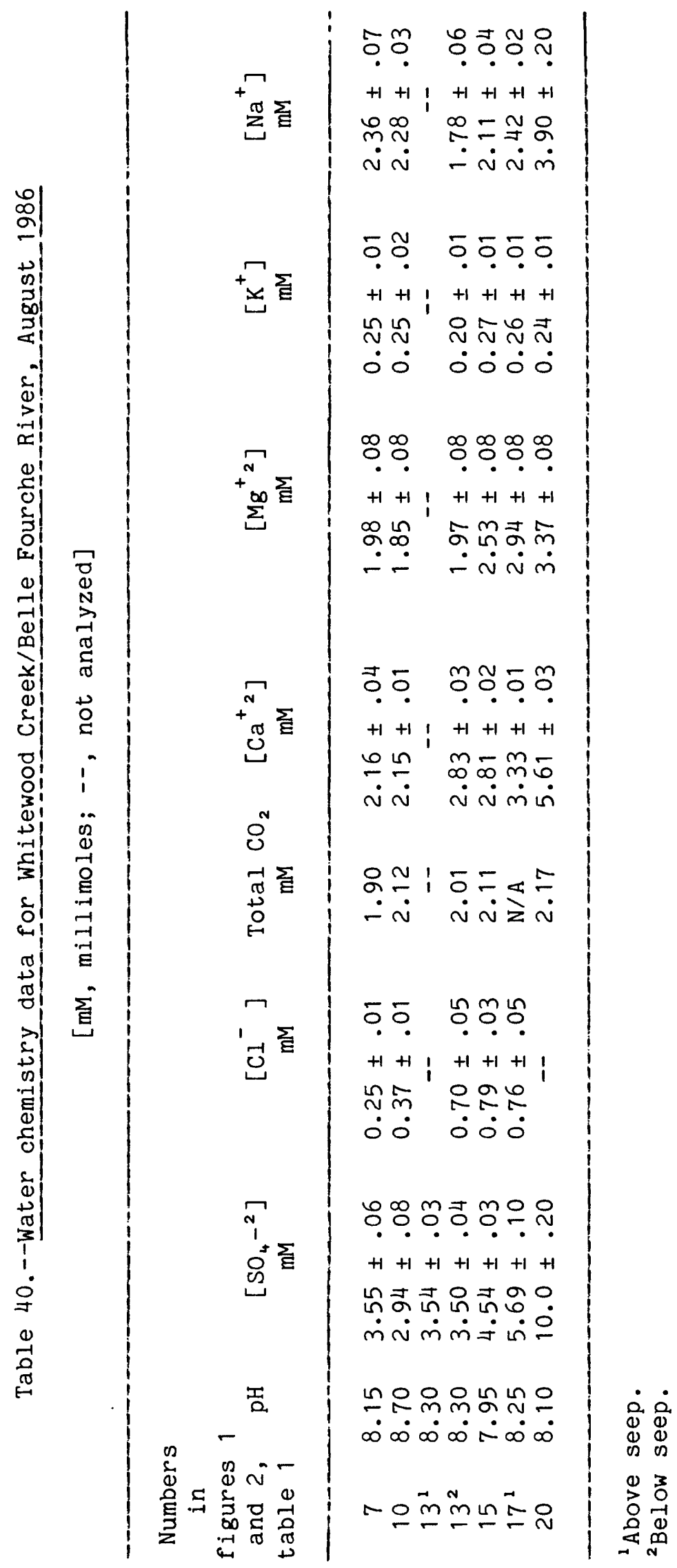



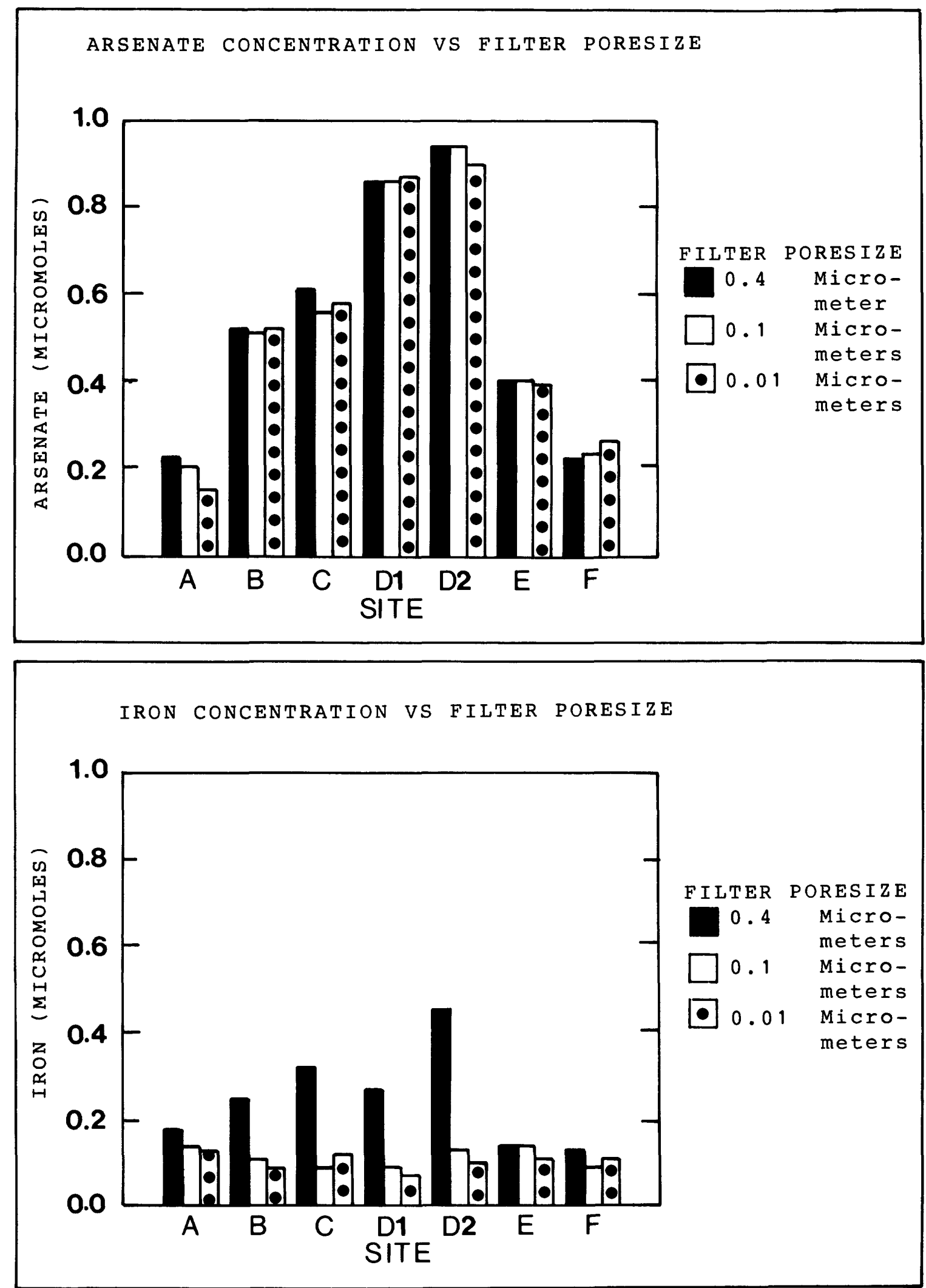

Figure 4.--Arsenate and iron versus filter poresize: Whitewood Creek/ Belle Fourche River, August 1986. (See table 40 for site code.) 
Table 41.--Arsenic and iron versus filter poresize, Whi tewood Creek/Belle Fourche River, August 1986

[ $\mu \mathrm{m}$, micrometers; $\mu \mathrm{M}$, micromoles]

\begin{tabular}{|c|c|c|c|c|c|c|}
\hline \multirow{2}{*}{$\begin{array}{l}\text { Numbers } \\
\text { in } \\
\text { figures } 1 \\
\text { and } 2, \\
\text { table } 1\end{array}$} & \multicolumn{3}{|c|}{ [As] $\mu \mathrm{M}$} & \multicolumn{3}{|c|}{$[\mathrm{Fe}] \mu \mathrm{M}$} \\
\hline & $<0.4 \mu \mathrm{m}$ & $<0.1 \mu \mathrm{m}$ & $<0.01 \mu \mathrm{m}$ & $<0.4 \mu \mathrm{m}$ & $<0.1 \mu \mathrm{m}$ & $<0.01 \mu \mathrm{m}$ \\
\hline 7 & $0.22 \pm .01$ & $0.23 \pm .03$ & $0.26 \pm .02$ & 0.13 & 0.09 & 0.11 \\
\hline 10 & $0.40 \pm .01$ & $0.40 \pm .01$ & $0.39 \pm .01$ & .14 & .14 & .11 \\
\hline $13^{1}$ & $0.94 \pm .03$ & $0.94 \pm .01$ & $0.90 \pm .02$ & .45 & .13 & .10 \\
\hline $13^{2}$ & $0.86 \pm .05$ & $0.86 \pm .02$ & $0.87 \pm .04$ & .27 & .09 & .07 \\
\hline 15 & $0.61 \pm .08$ & $0.56 \pm .05$ & $0.58 \pm .07$ & .32 & .09 & .12 \\
\hline $17^{1}$ & $0.52 \pm .01$ & $0.51 \pm .01$ & $0.52 \pm .01$ & .25 & .11 & .09 \\
\hline 20 & $0.22 \pm .01$ & $0.20 \pm .01$ & $0.15 \pm .02$ & .18 & .14 & .13 \\
\hline
\end{tabular}

${ }^{1}$ Above seep.

${ }^{2}$ Below seep.

\section{REFERENCES}

Culbertson, C.W., Zehnder, A.J.B., Oremland, R.0., 1981, Anaerobic oxidation of acetylene by estuarine sediments and enrichment cultures: Applied Environmental Microbiology, v. 41, p. 396.

Ficklin, W.H., 1983, Separation of arsenic (III) and arsenic (V) in ground waters by ion exchange: Talanta, v. 30, p. 371 .

Stookey, L.L., 1970, Ferrozine - A new spectrophotometric reagent for iron: Analytical Chemistry, v. 42, p. 779. 


\section{Chapter G. Ground-Water Geochemistry along Whitewood Creek}

By Christopher C. Fuller, James A. Davis, and Regina G. Claypool-Frey

\section{SAMPLE COLLECTION AND HANDLING PROCEDURES}

Ground-water samples were collected at the Berger and Sheeler sites (fig. 1 and table 1) along Whitewood Creek in August 1986. Sampling piezometers installed at these sites by an earlier study (Cherry and others, 1985) were used. The screen depth and associated aquifer materials of the wells sampled are presented in table 42.

Table 42.--Screen depth and aquifer material for ground-water wells sampled along Whitewood Creek, August 1986

Well

number ${ }^{1}$

Aquifer material ${ }^{1}$

Screen depth ${ }^{1}$ (feet)

$\begin{array}{cl}\text { Berger site } & \\ \text { B-1-4 } & \text { Tailings } \\ \text { B-1-2 } & \text { Alluvium } \\ \text { B-1-3 } & \text { Alluvium } \\ \text { B-2-1 } & \text { Tailings } \\ \text { B-2-2 } & \text { Alluvium } \\ \text { B-2-3 } & \text { Alluvium } \\ \text { B-2-24 } & \text { Shale ? } \\ \text { B-4 } & \text { Tailings }\end{array}$

$\begin{array}{ll}5.5 & -6.5^{2} \\ 13 & -14 \\ 14 & -15 \\ 10.5 & -11.5^{2} \\ 11.5 & -12.5^{2} \\ 14 & -15 \\ 12 & -24 \\ 4 & -6.5\end{array}$

Sheeler site S-4

Tailings/alluvium

$10-17$

${ }^{1}$ From Cherry and others, 1985.
${ }^{2}$ Insufficient water to sample.

Samples were collected by pumping through one-fourth-inch outside diameter nylon tubing inserted down the well casing to the base of the screened depth. Prior to pumping, the water depth and level in each well was measured. Wells were developed for sampling by pumping with a peristaltic pump ( 100 to $200 \mathrm{~mL} / \mathrm{min}$ ) three to four times the calculated volume of standing water in the casing. Samples were not collected from wells that were not able to produce at this flow rate.

Ground-water flow from the pump was then directed through an in-line teflon flow cell containing a combination $\mathrm{pH}$ electrode, with the pH value recorded after a stable reading was obtained. Total dissolved $\mathrm{CO}_{2}$ samples were drawn by syringe from the outlet of the flow cell and immediately injected into septa-capped vials. The flow was then directed into the upper chamber of a 47-mm diameter polycarbonate filter tower which had been entirely 
purged with $\mathrm{N}_{2}$. A measured volume was then filtered by vacuum through a 0.4- um cellulose nitrate membrane. Concentrated HCL was injected into the filtrate in the lower reservoir to obtain a final concentration of 1 percent $(\mathrm{v} / \mathrm{v})$. Sampling and filtering by this method allowed acidification of the filtrate before exposure to the atmosphere and, thus, eliminated problems resulting from oxidation of the sample. Aliquots of the acidified filtrate were then transferred into polypropylene bottles. Aliquots for major iron, total iron, $\mathrm{Fe}^{2}+$, and total arsenic determinations were refrigerated until analyzed. An aliquot for $\mathrm{As}^{3}-\mathrm{As}^{3}$ speciation was immediately processed by the anion exchange technique of Ficklin (1983) with the fractionated samples then refrigerated. All sample containers and sampling apparatus were acidwashed prior to use in the field.

\section{ANALYTICAL TECHNIQUES AND RESULTS}

Major cations $\left(\mathrm{Ca}^{2}{ }^{+}, \mathrm{Mg}^{2}, \mathrm{~K}^{+}, \mathrm{Na}^{+}\right)$were determined by flame atomic absorption. Sulfate was determined by ion chromatography employing a conductivity detector and a borate-gluconate buffer mobile phase. Chloride was not determined since $\mathrm{HCL}$ was used for sample preservation. Total and ferrous iron were determined colorimetrically by the ferrozine method (Stookey, 1970) with ferric iron calculated by difference. Total As in the unfractionated aliquots, and arsenite and arsenate in the fractionated aliquots were determined by graphite furnace atomic absorption utilizing similar operating conditions to Ficklin's (1983). Total dissolved $\mathrm{CO}_{2}$ was determined by gas chromatography of the sample vial headspace after acidification (Culbertson and others, 1981). Results are presented in table 43.

\section{REFERENCES}

Cherry, J.A., Morel, F.M.M., Rouse, J.V., Schnoor, J.L., Wolman, M.G., 1985, Mineral and energy resource series: Colorado School of Mines.

Culbertson, C.W., Zehnder, A.J.B., Oremland, R.O., 1981, Applied environmental microbiology: v. 41, p. 396.

Ficklin, W.H., 1983, Separation of arsenic (III) and arsenic (V) in ground waters by ion exchange: Talanta, v. 30, p. 371 .

Stookey, L.L., 1970, Ferrozine--A new spectrophotometric reagent for iron: Analytical Chemistry, v. 42, p. 779. 


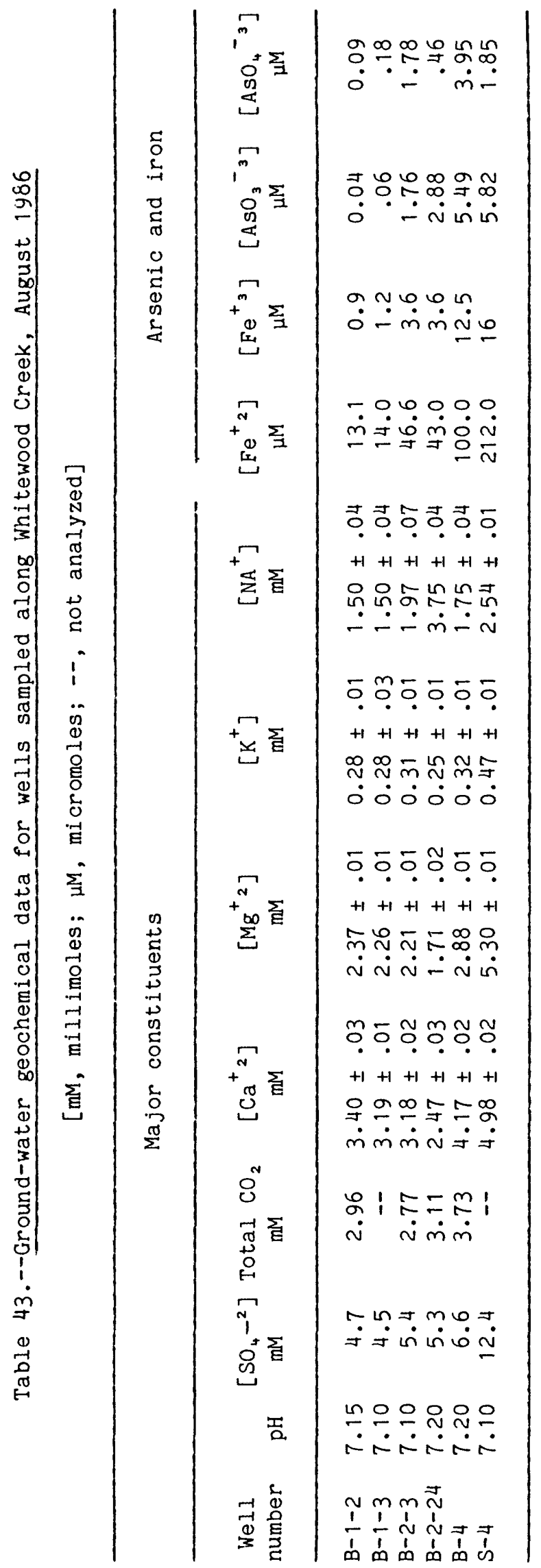




\section{Chapter H. Colloids in Seeps and Springs along Whitewood Creek}

By Terry F. Rees and James F. Ranville

\section{INTRODUCTION}

Colloids are particulates, droplets, gas bubbles, or films which have at least one dimension in the range, 0.001 to $1 \mu \mathrm{m}$, and are dispersed in a second continuous phase such as water. When pollutants become associated (sorbed, co-precipitated) with colloids, the pollutant's transport is no longer controlled by simple solution thermodynamics and kinetics. Rather, their transport is controlled by that of the host colloid. Very little is known about the relative importance of colloids in the transport of heavy metals in the geosphere. The U.S. Geological Survey has identified two seeps along Whitewood Creek in South Dakota as possible systems in which colloidfacilitated transport of heavy metals may be occurring.

First-year investigations (1986) were performed at the Sheeler site seeps located on Whitewood Creek approximately $1 \mathrm{~km}$ from its confluence with the Belle Fourche River near Newell, South Dakota (fig. 1, table 1). The seeps are a result of downcutting through a historic meander of Whitewood Creek that was filled with tailings washed down from the Homestake Mine near Lead, South Dakota. After the meander filled, Whitewood Creek eroded through the meander sediments and into the underlying shale bedrock. Ground water percolates through the tailings and fluvial sediments until it encounters the relatively impermeable bedrock. Further flow is then directed laterally across the surface of the shale, until the water discharges at the land surface near the creek. Emergent ground water then flows over the shale into small quiescent pools prior to mixing with the stream water.

\section{SAMPLE COLLECTION AND HANDLING PROCEDURES}

Samples for this study were collected during August 1986 from Whitewood Creek immediately upstream and downstream from the seeps, from a quiescent pool, from water flowing over the face of the outcrop, and from several inches into the outcrop. The subsurface seep water was collected by pushing a PVC tube several inches into the shale outcrop. The end of the tube was protected by screening to prevent it being clogged while being positioned. Water was then allowed to flow through the tube for about an hour prior to sample collection. It is believed that this procedure precludes oxidation of the ground water prior to collection. The surficial seep samples were collected using a peristaltic pump, placing the inlet tube just beneath the surface of the water. The upstream sample was a grab sample. Field-collected data are given in table 44. Due to a breakdown of the field pH meter, no pH measurements are available. 
Table 44.--Specific conductance and temperature measured at the Sheeler site, Whitewood Creek, during August 1986

\begin{tabular}{lcc}
$\begin{array}{c}\text { Sample } \\
\text { location }\end{array}$ & $\begin{array}{c}\text { Specific conductance } \\
\text { (microsiemens) }\end{array}$ & $\begin{array}{c}\text { Temperature } \\
\left({ }^{\circ} \mathrm{C}\right)\end{array}$ \\
\hline $\begin{array}{l}\text { Whitewood Creek upstream from site } \\
\text { Surface flow over outcrop }\end{array}$ & 1,200 & 14.0 \\
Quiescent pool & 2,200 & 14.0 \\
Whitewood Creek downstream from site & 2,000 & 19.2 \\
\hline
\end{tabular}

Analyses for various chemical components were performed on filtered $(0.40-, 0.10-$, and $0.05-\mu \mathrm{m})$ and unfiltered fractions. Filtered samples were processed using $147-\mathrm{mm}$ barrel filters fitted with Nucleopore polycarbonate membrane filters. All filtrations were performed under nitrogen to minimize oxidation. Samples for cations were immediately acidified to $\mathrm{pH}<2$ with reagent-grade nitric acid. Samples for anions were left unacidified.

\section{ANALYTICAL PROCEDURES AND RESULTS}

Metals were determined by inductively-coupled plasma analysis (Garbarino and Taylor, 1979) except for arsenic, silica, and aluminum. These metals were determined by graphite furnace (As) and nitrous oxide flame (Si, Al) atomic absorption spectrometry (Fishman and Friedman, 1985). Sulfate was determined by the turbidometric procedure given in Fishman and Friedman (1985) except a photon correlation spectrometer was used to measure scattering intensity, instead of the traditional absorption method. Analytical data are given in table 45. All analyses are \pm 10 percent.

The presence of suspended colloids was determined using photon correlation spectrometry (PCS) (Rees, 1987). PCS gives information about colloid concentrations (from the integrated scattering intensity) and colloid size distributions. Samples for PCS analysis were collected using the same nitrogen filtration apparatus as used for the water chemistry samples. These samples were collected and sealed in glass ampules under nitrogen atmosphere, and were analyzed 2 to 4 hours after collection. PCS data is $g$ iven in table 46.

Membranes used for the filtrations were retained and examined using scanning electron microscopy coupled with energy dispersive $x$-ray analysis. Photomicrographs and $x$-ray spectra for representative particles retained on the filters are given in figures 5 to 13. 


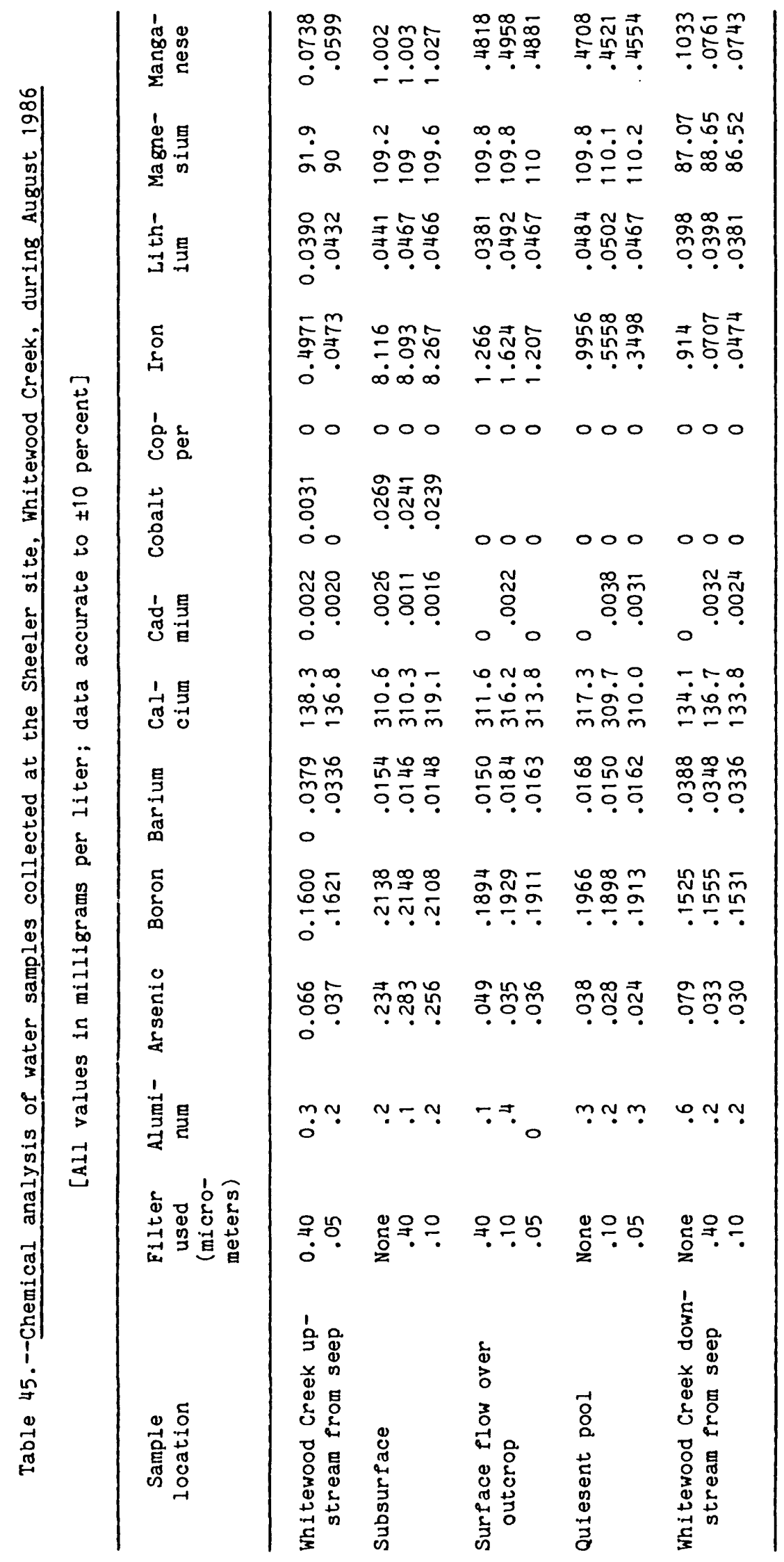




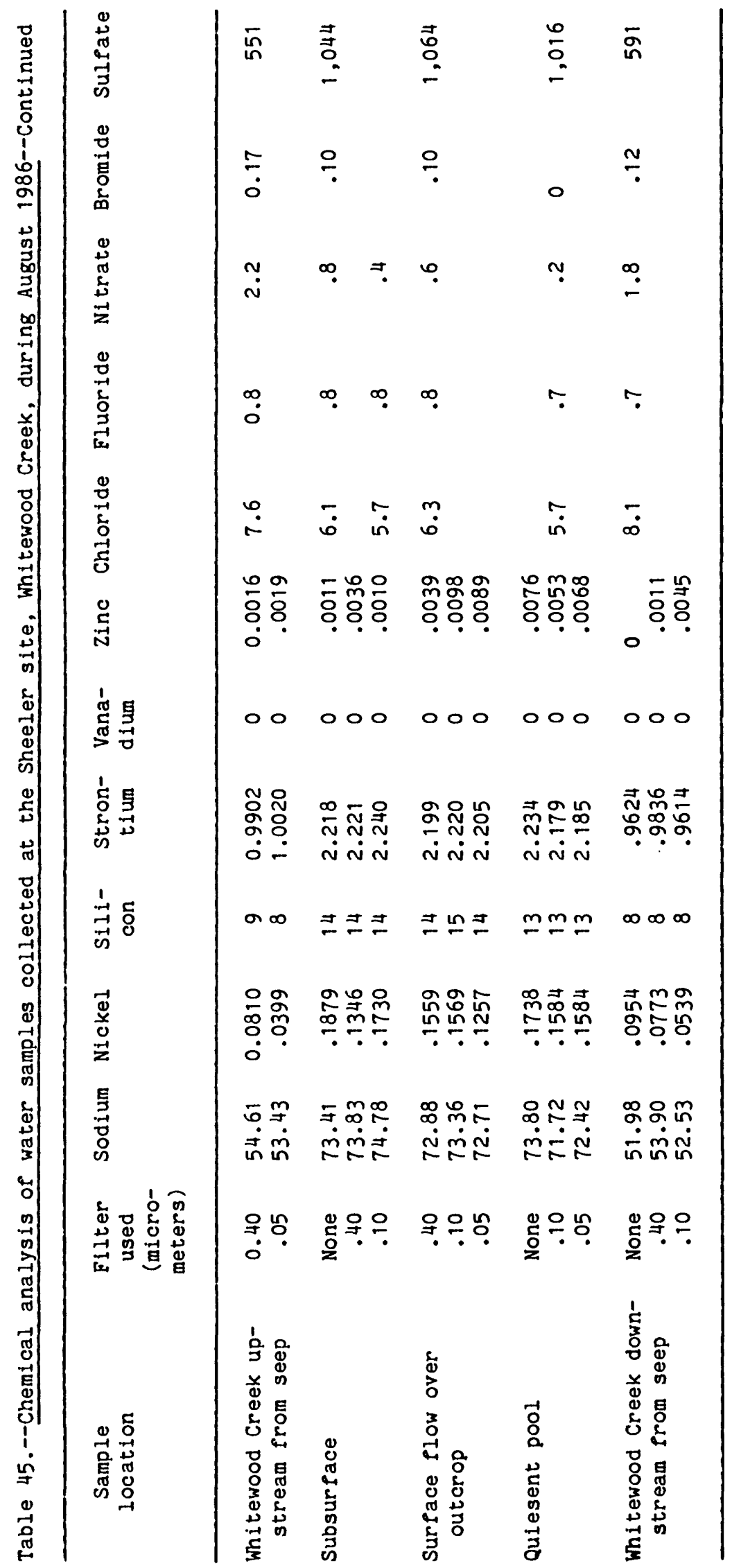




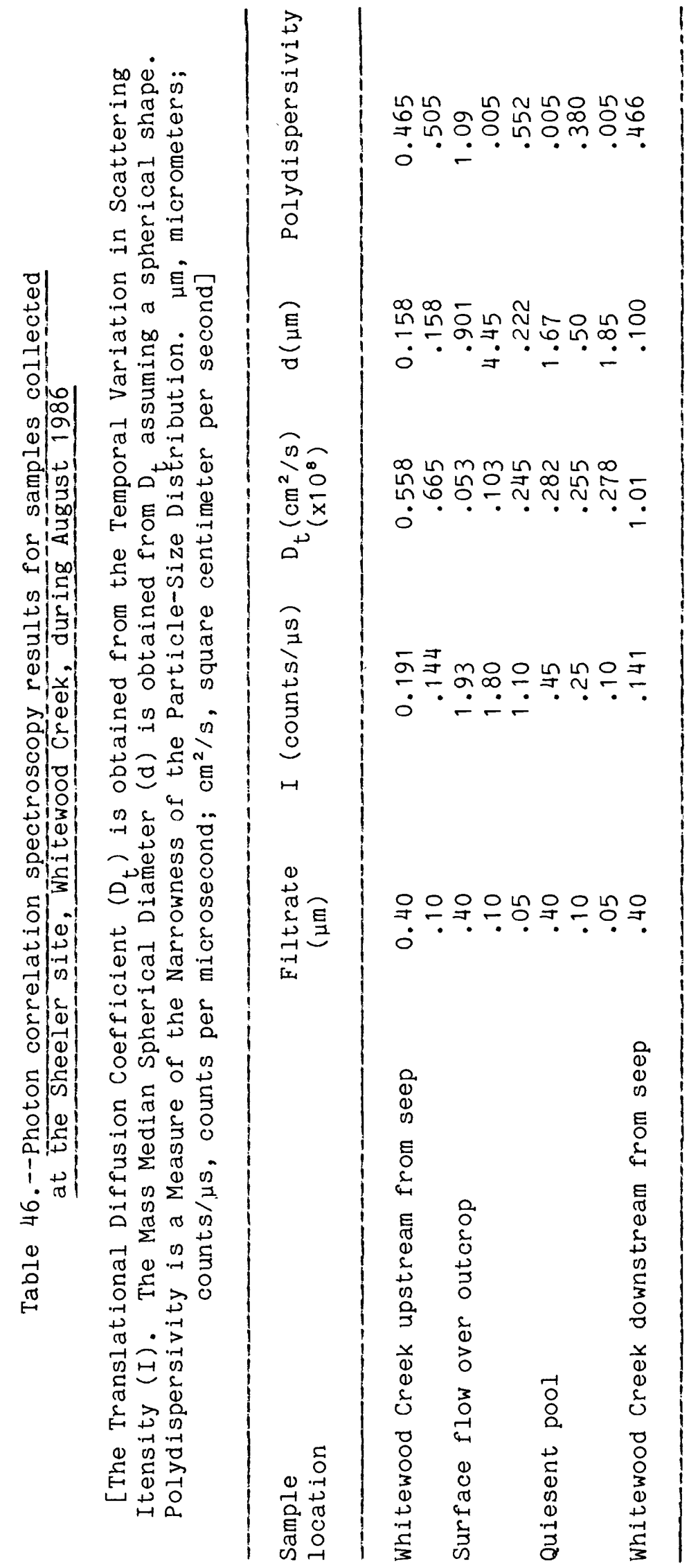




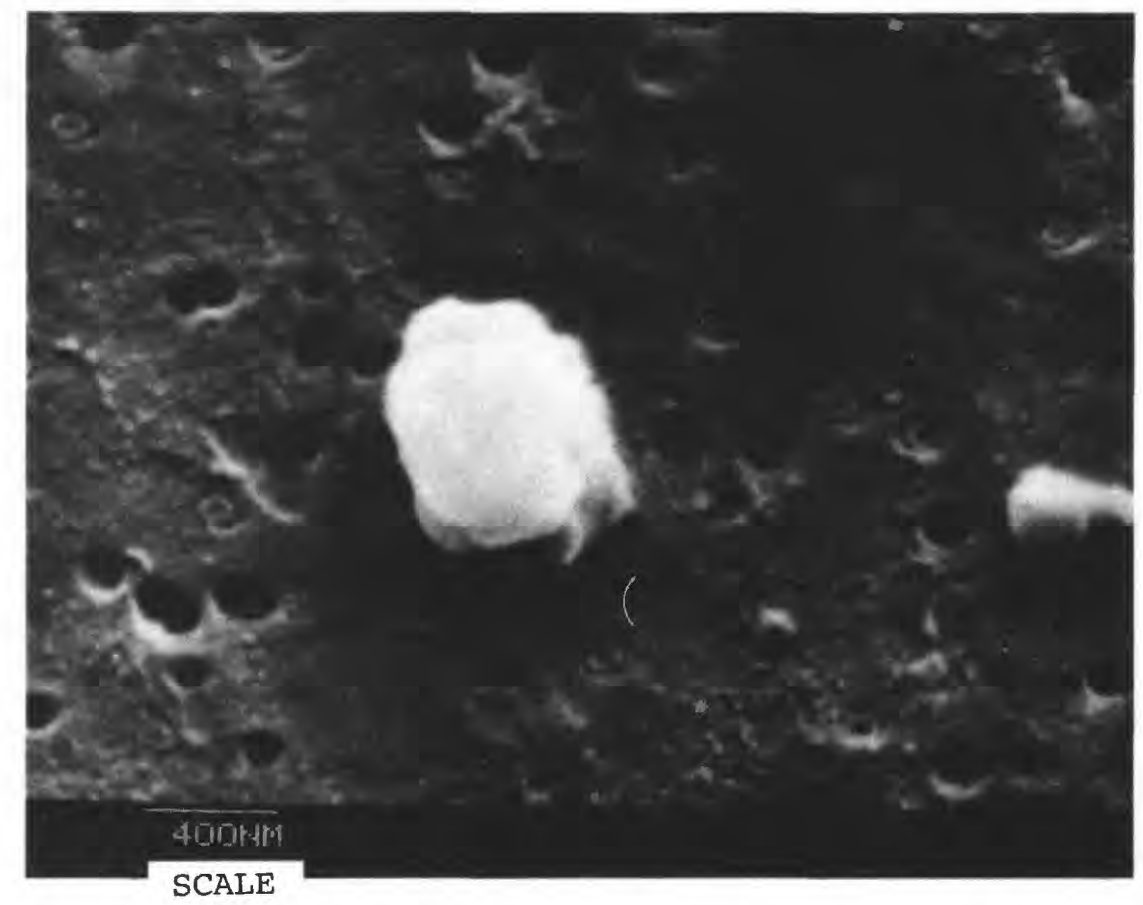

Figure 5A.--Scanning-electron photomicrograph of particle 01 retained on $100-\mathrm{nm}$ filter, from water sample collected from surface flow at the Sheeler Seep, Whitewood Creek, August 1986.

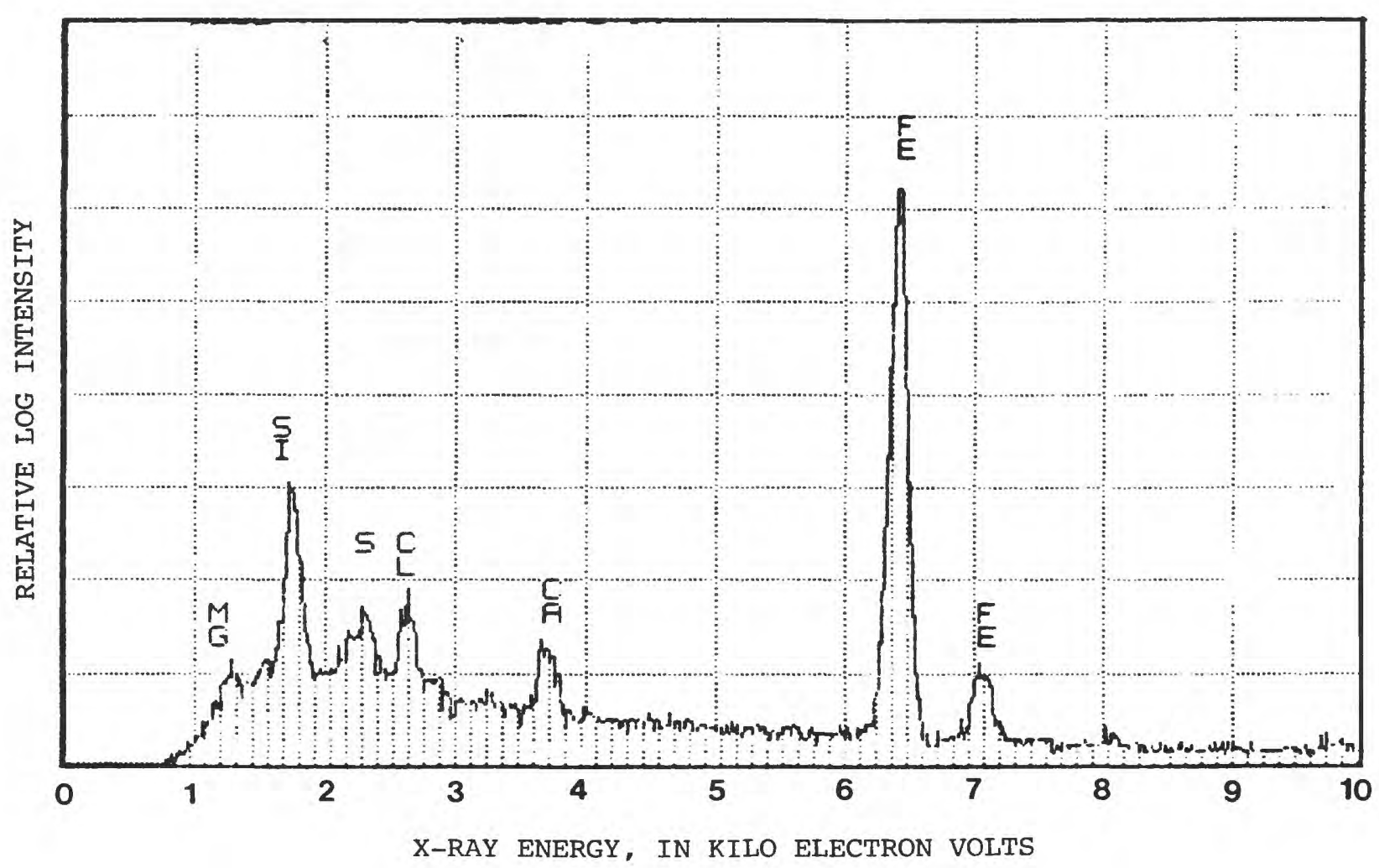

Figure 5B.--Energy dispersive X-ray analysis of particle 01 retained on 100-nm filter, from water sample collected from surface flow at the Sheeler Seep, Whitewood Creek, August 1986. 


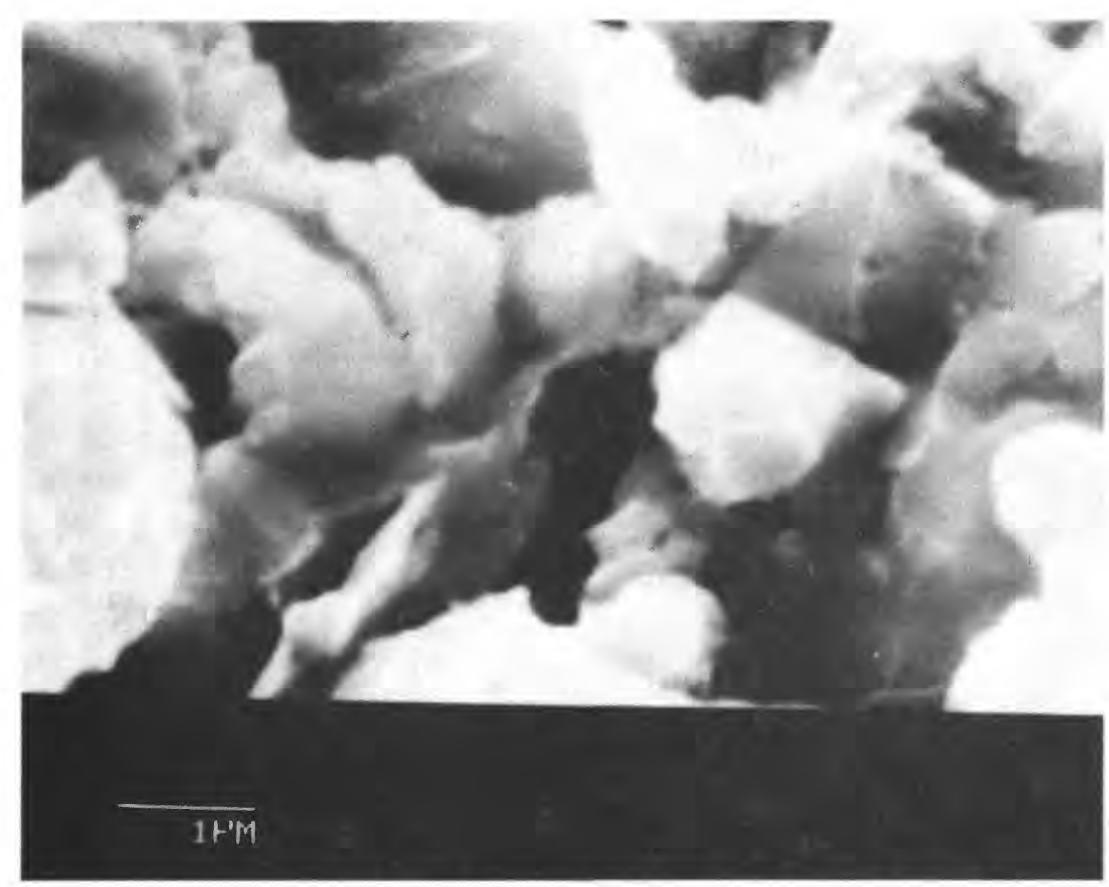

Figure 6A.--Scanning-electron photomicrograph of particle 02 retained on 450-NM filter, from water sample collected from the pool at the Sheeler seep, Whitewood Creek, August 1986.

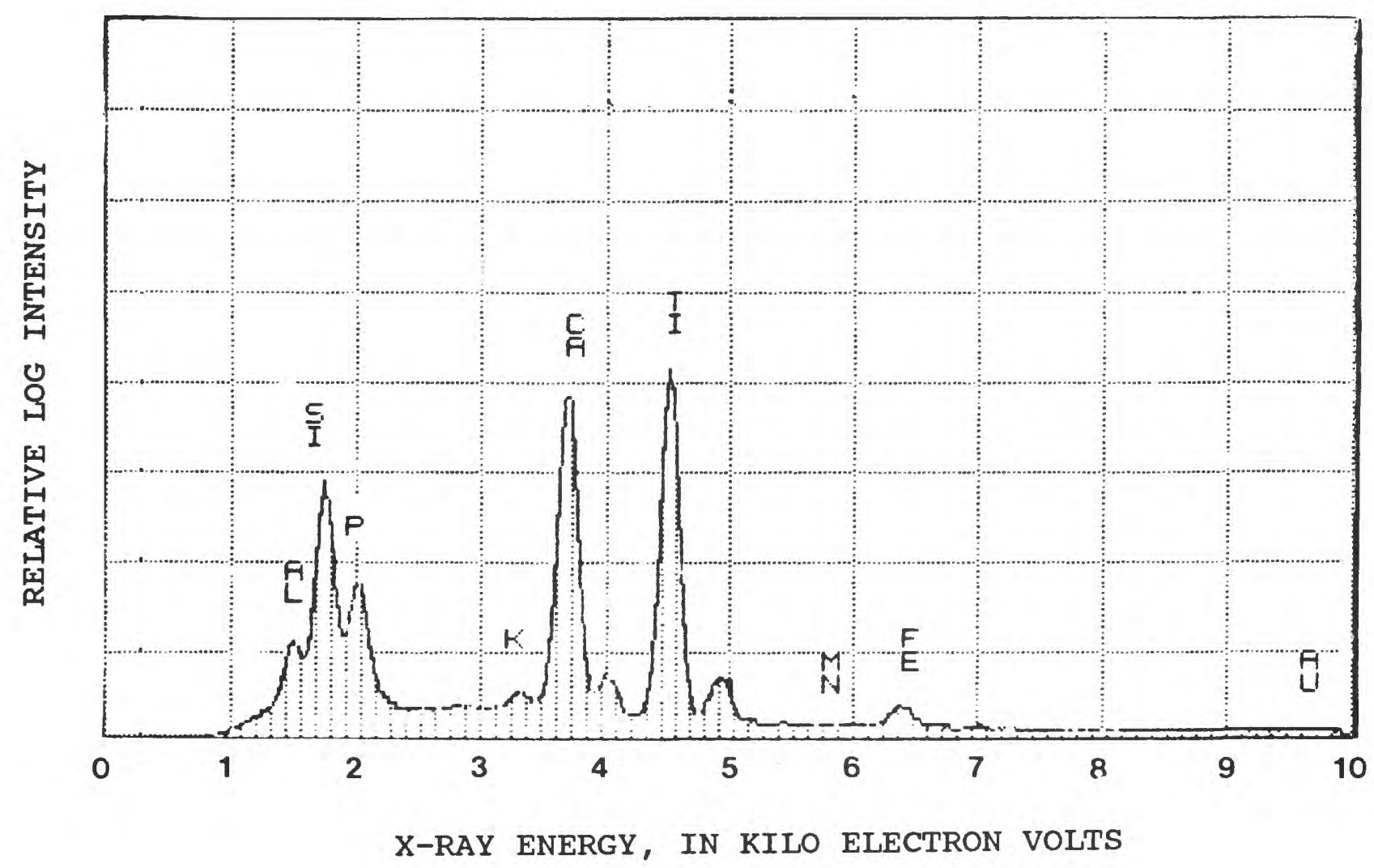

Figure 6B.--Energy dispersive $\mathrm{X}$-ray analysis of particle 02 retained on 450-NM filter, from water sample collected from the pool at the sheeler seep, Whitewood Creek, August 1986. 


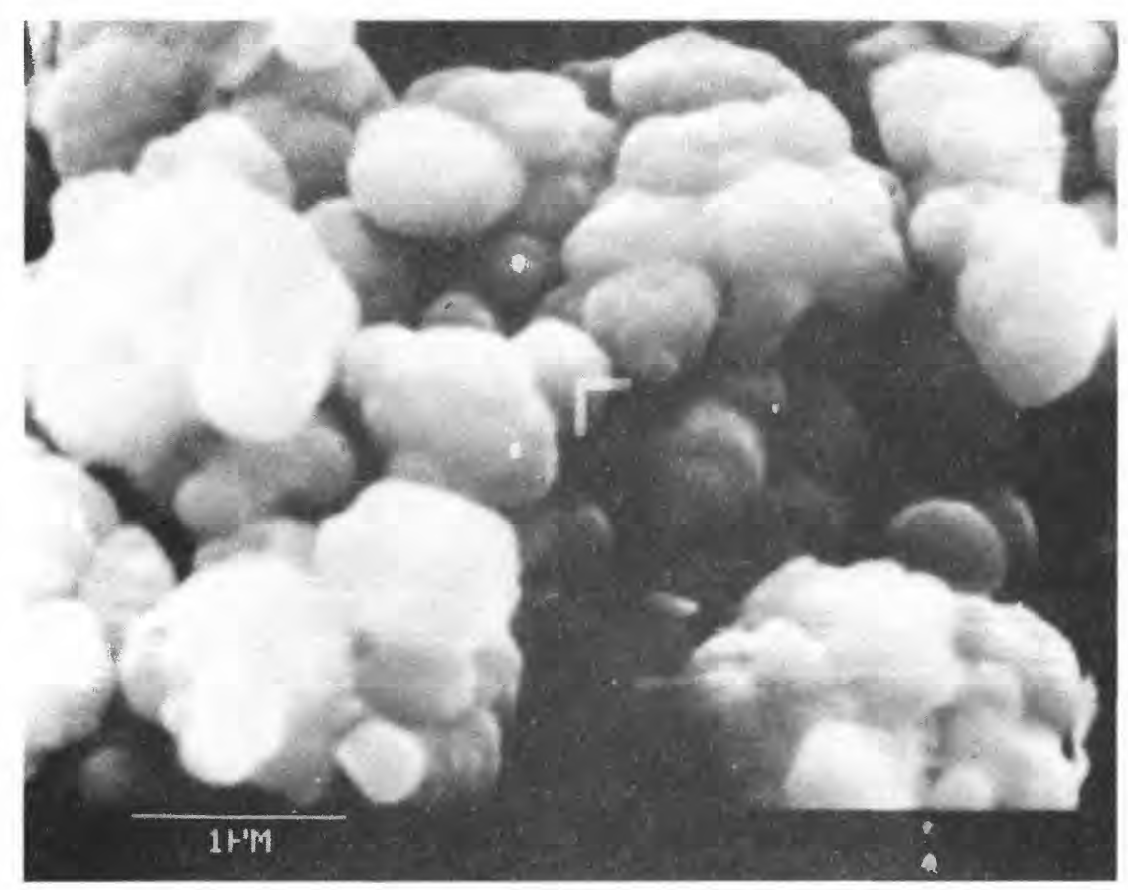

Figure 7A.--Scanning-electron photomicrograph of particle 03 retained on 450-NM filter, from water sample collected from the pool at the sheeler seep, Whitewood Creek, August 1986.

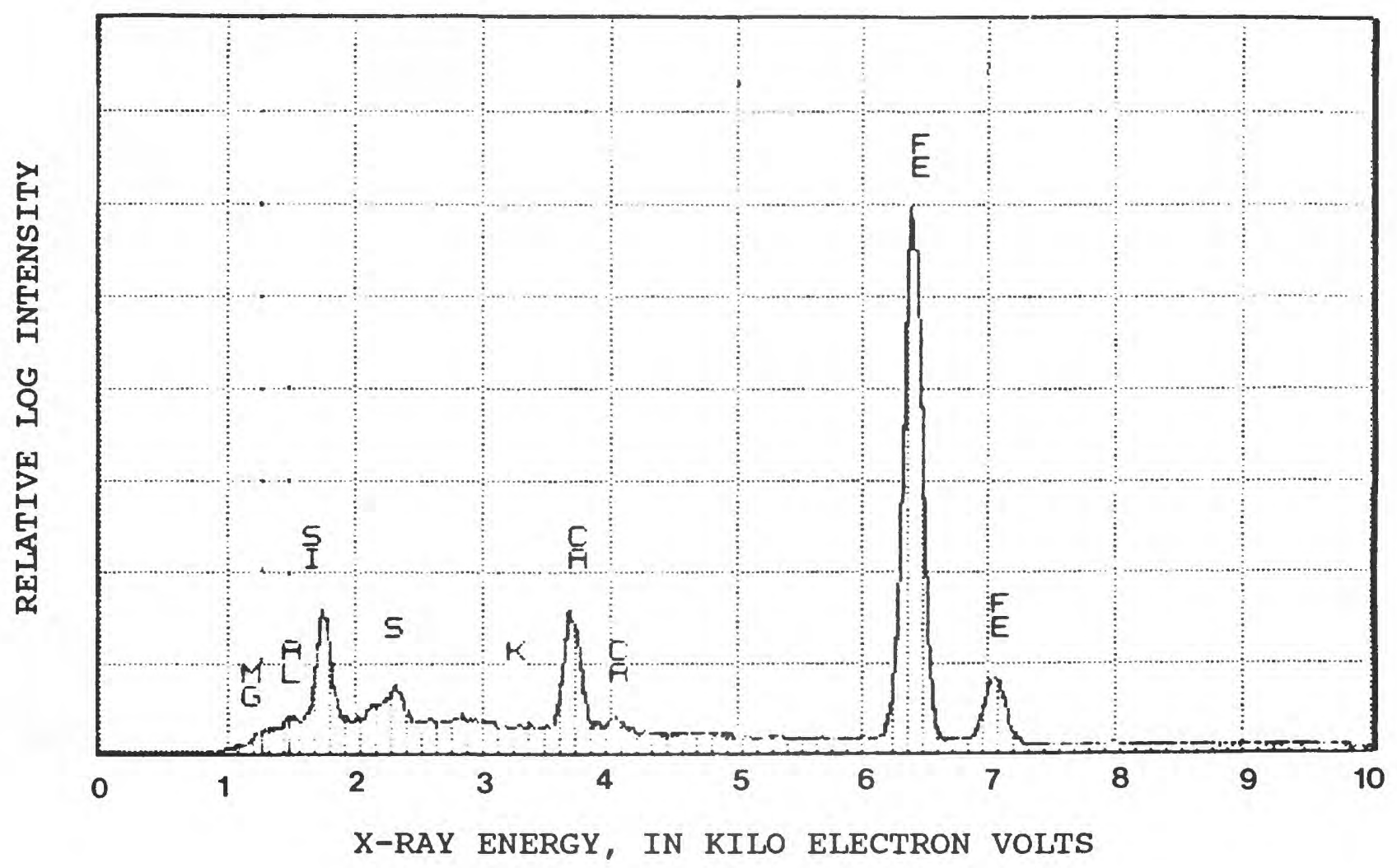

Figure 7B.--Energy dispersive $X$-ray analysis of particle 03 retrained on 450-NM filter, from water sample collected from the pool at the sheeler seep, Whitewood Creek, August 1986. 


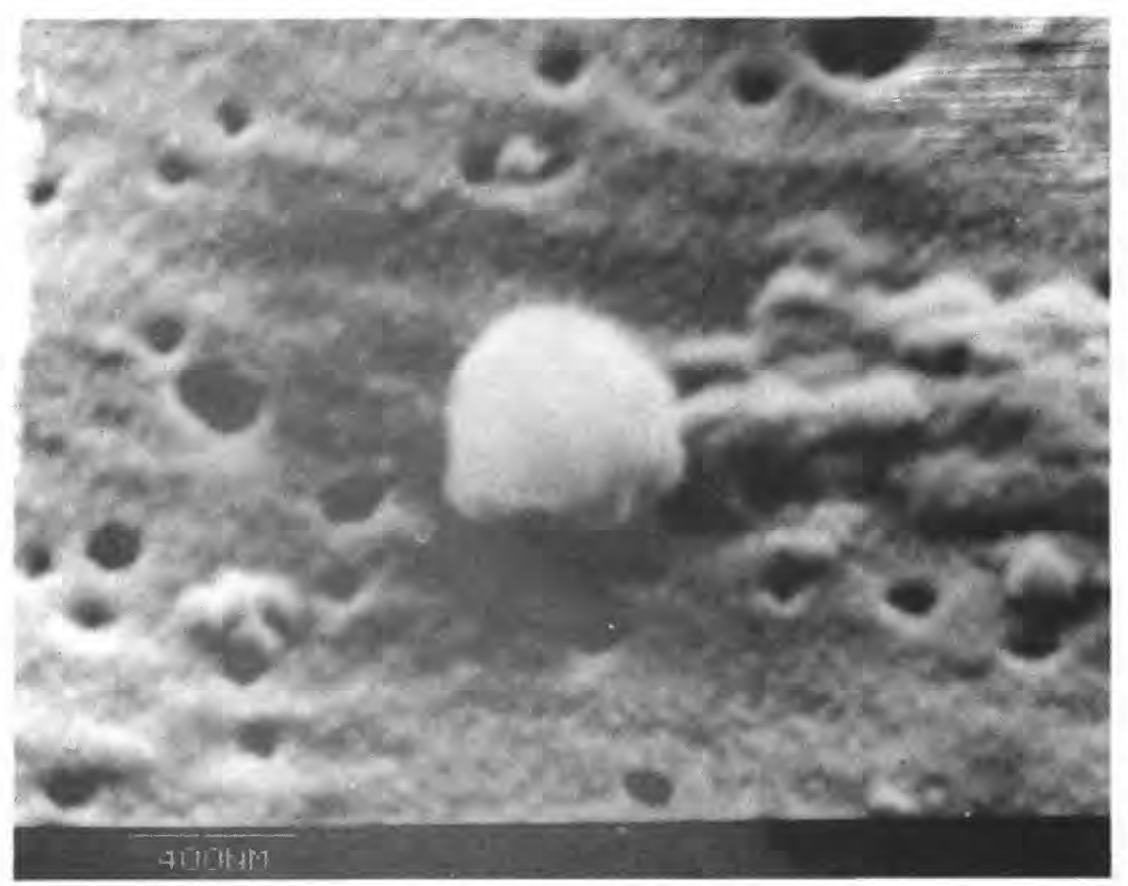

Figure 8A.--Scanning-electron photomicrograph of particle 04 retained on 100-NM filter, from water sample collected from the pool at the sheeler seep, Whitewood Creek, August 1986.

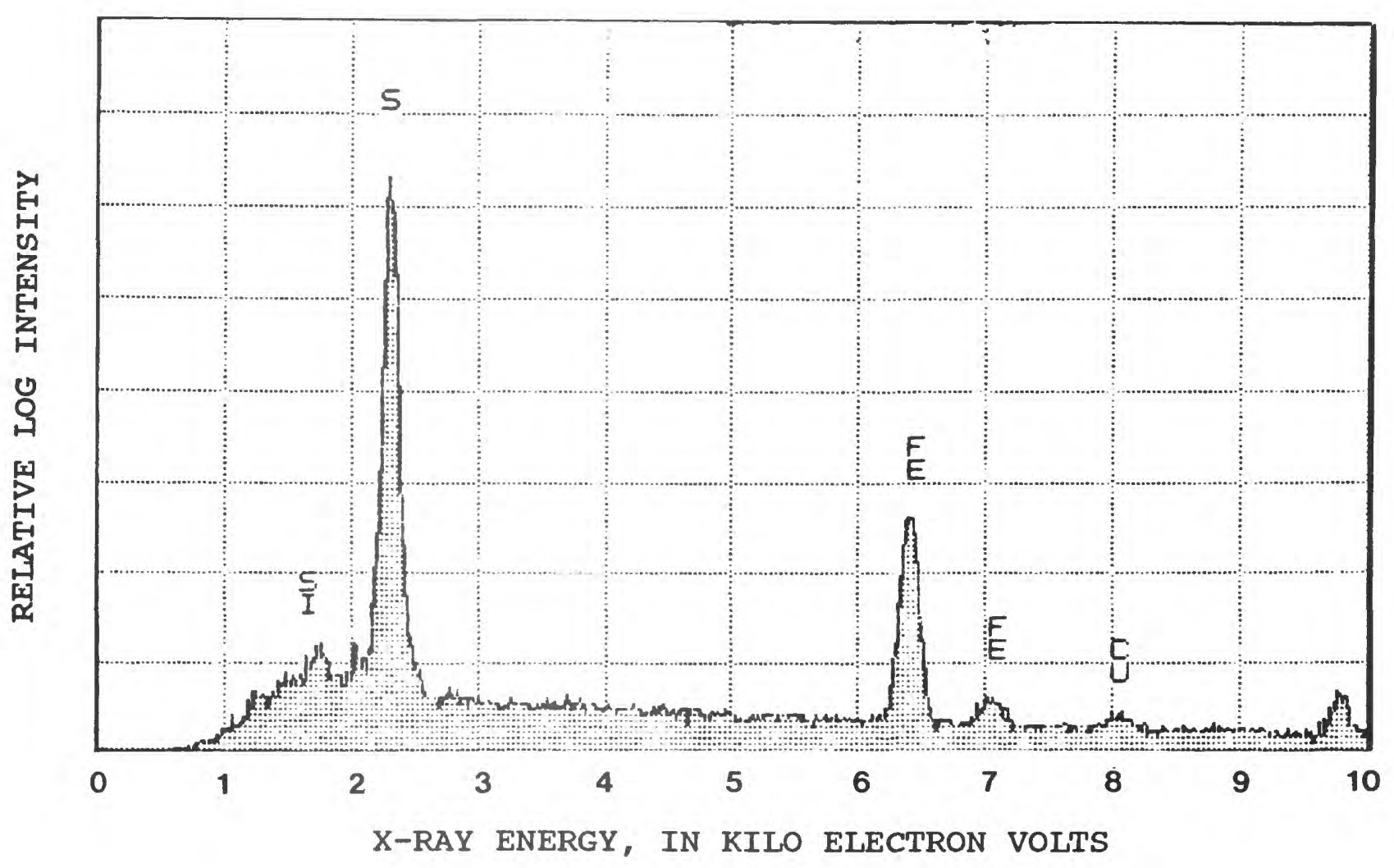

Figure 8B.--Energy dispersive X-ray analysis of particle 04 retained on 100-NM filter, from water sample collected from the pool at the sheeler seep, Whitewood Creek, August 1986. 


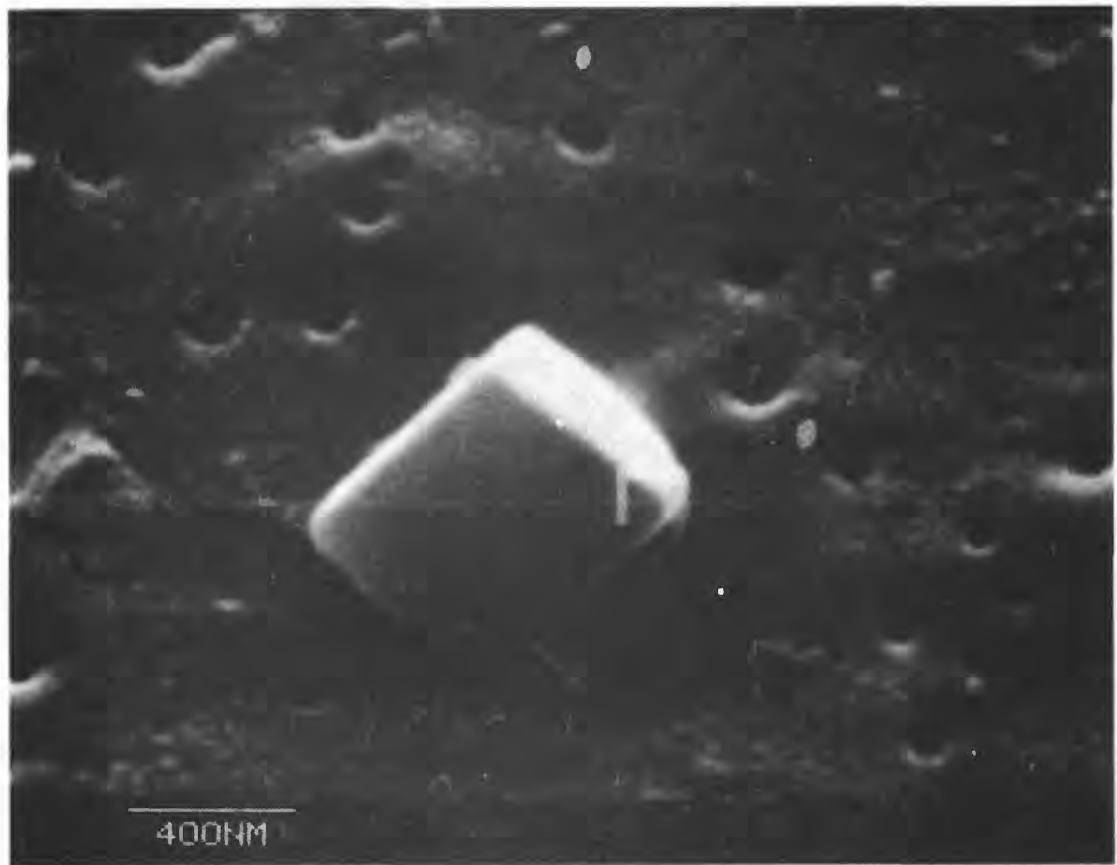

Figure 9A.--Scanning-electron photomicrograph of particle 05 retained on 100-NM filter, from water sample collected from the pool at the Sheeler seep, Whitewood Creek, August 1986.

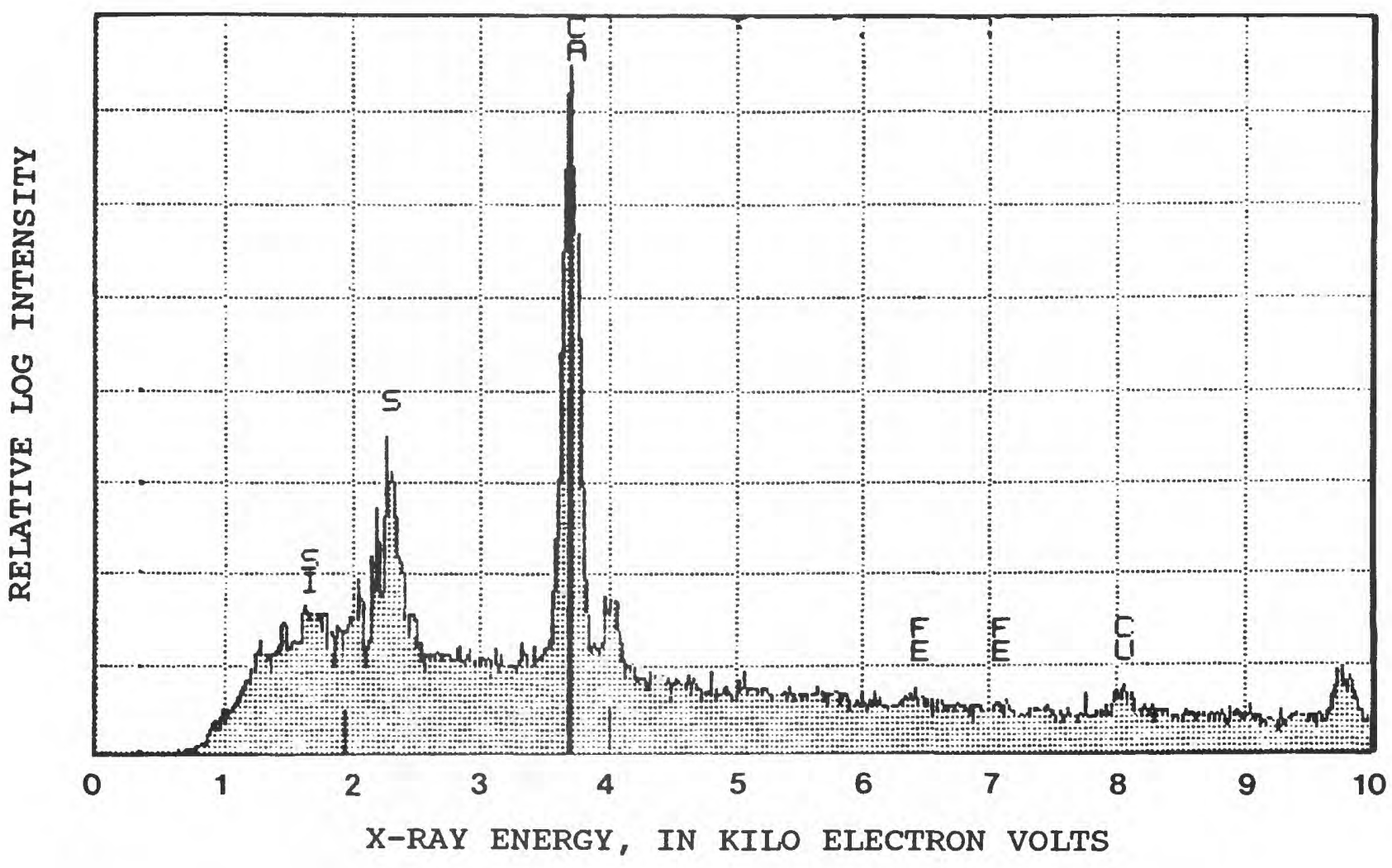

Figure 9B.--Energy dispersive X-ray analysis of particle 05 Retained on 100-NM Filter, from water sample collected from the pool at the Sheeler Seep, Whitewood Creek, August 1986. 


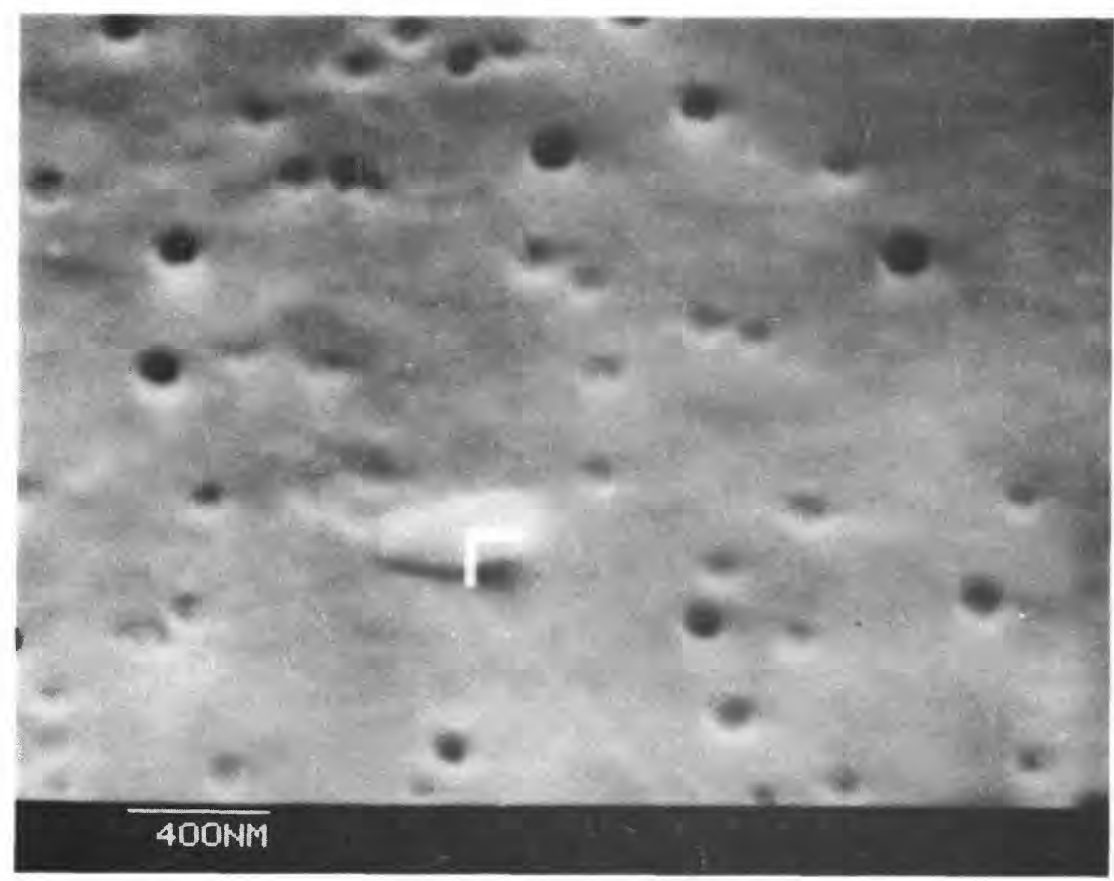

Figure 10A.--Scanning-electron photomicrograph of particle 06 retained on 100-NM filter, from water sample collected from the pool at the sheeler seep, Whitewood Creek, August 1986.

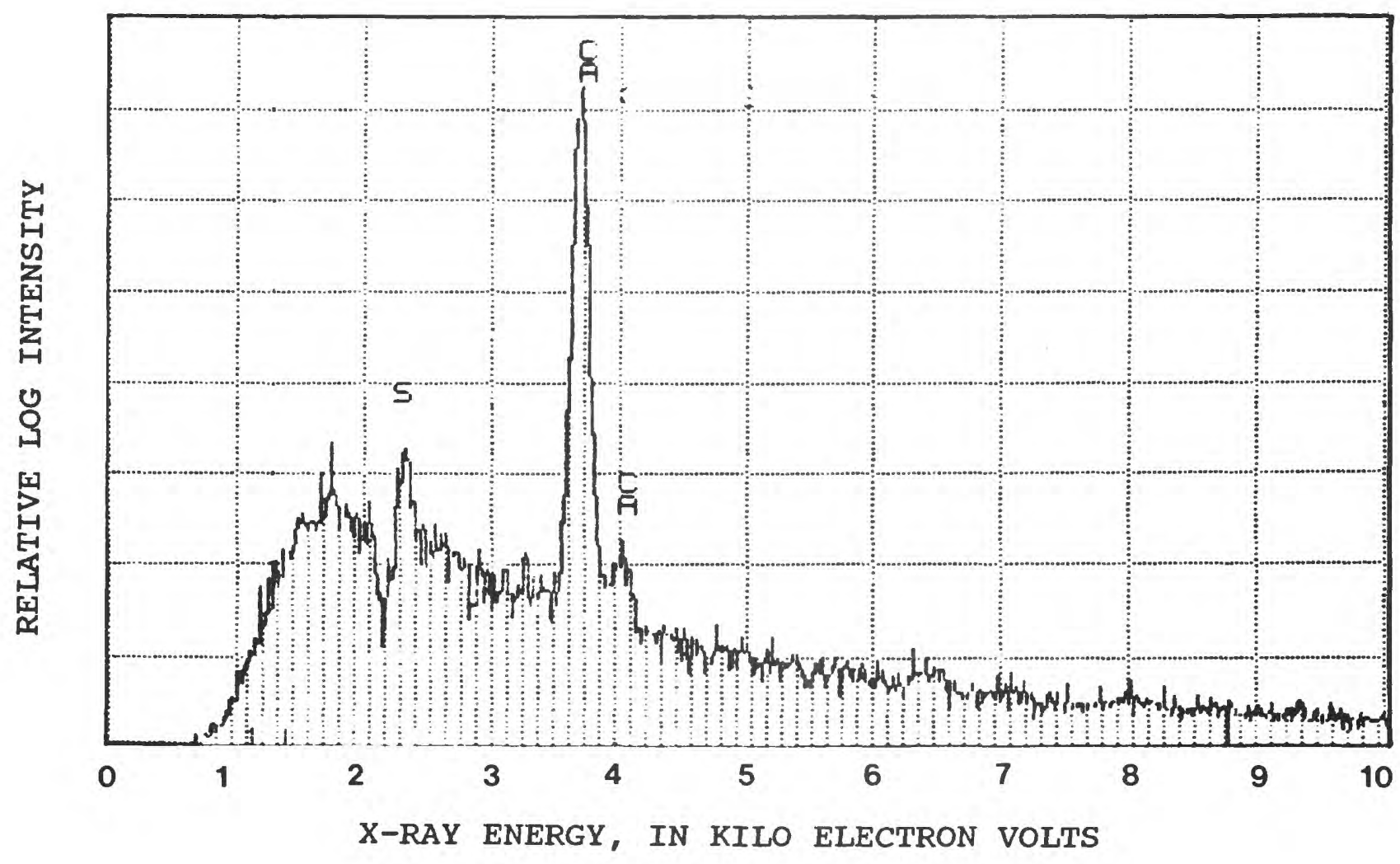

Figure 10B.--Energy dispersive X-ray analysis of particle 06 retained on 100-NM filter, from water sample collected from the pool at the Sheeler seep, Whitewood Creek, August 1986. 


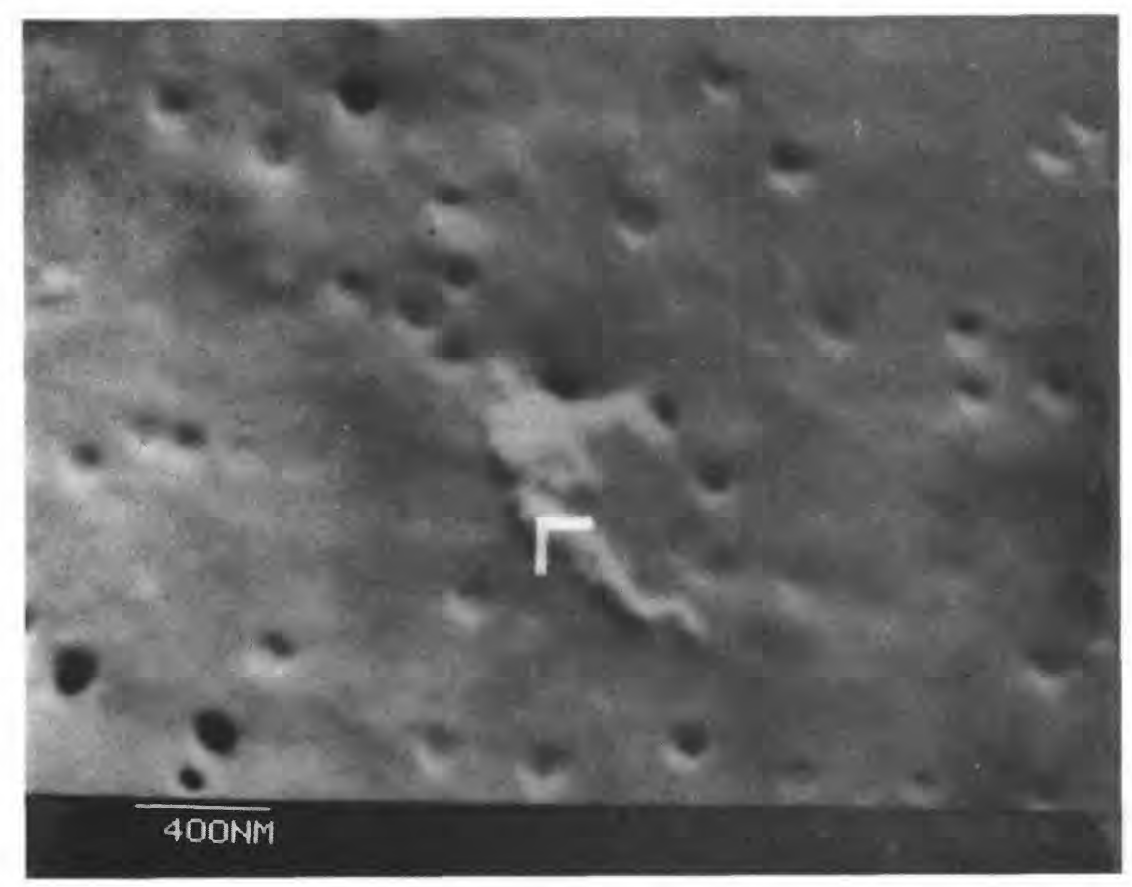

Figure 11A.--Scanning-electron photomicrograph of particle 07 retained on $100 \mathrm{NM}$ filter, from water collected from the pool at the Sheeler seep, Whitewood Creek, August 1986.

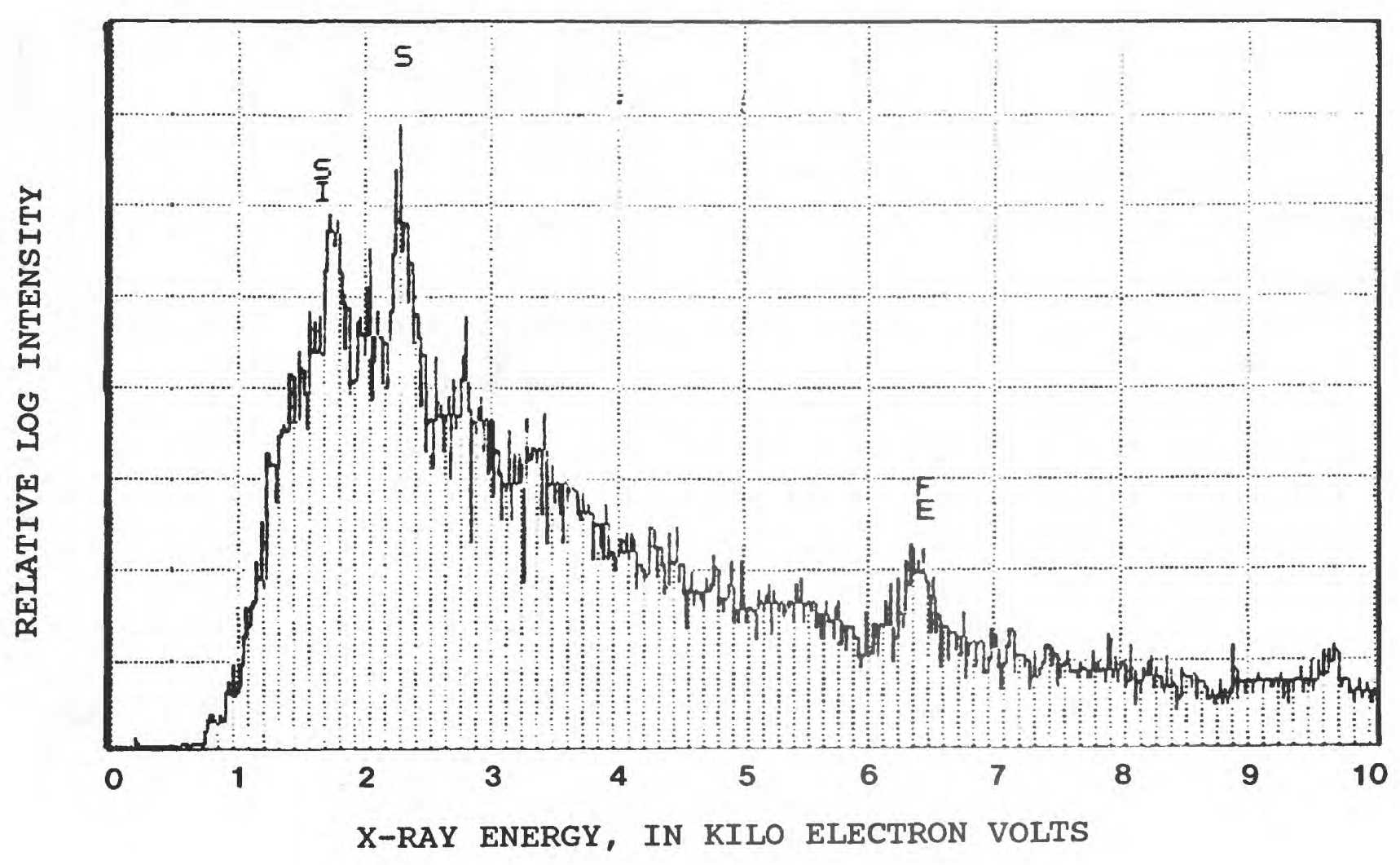

Figure 11B.--Energy dispersive $X$-ray analysis of particle 07 retained on 100-NM filter, from water collected from the pool at the Sheeler Seep, Whitewood Creek, August 1986. 


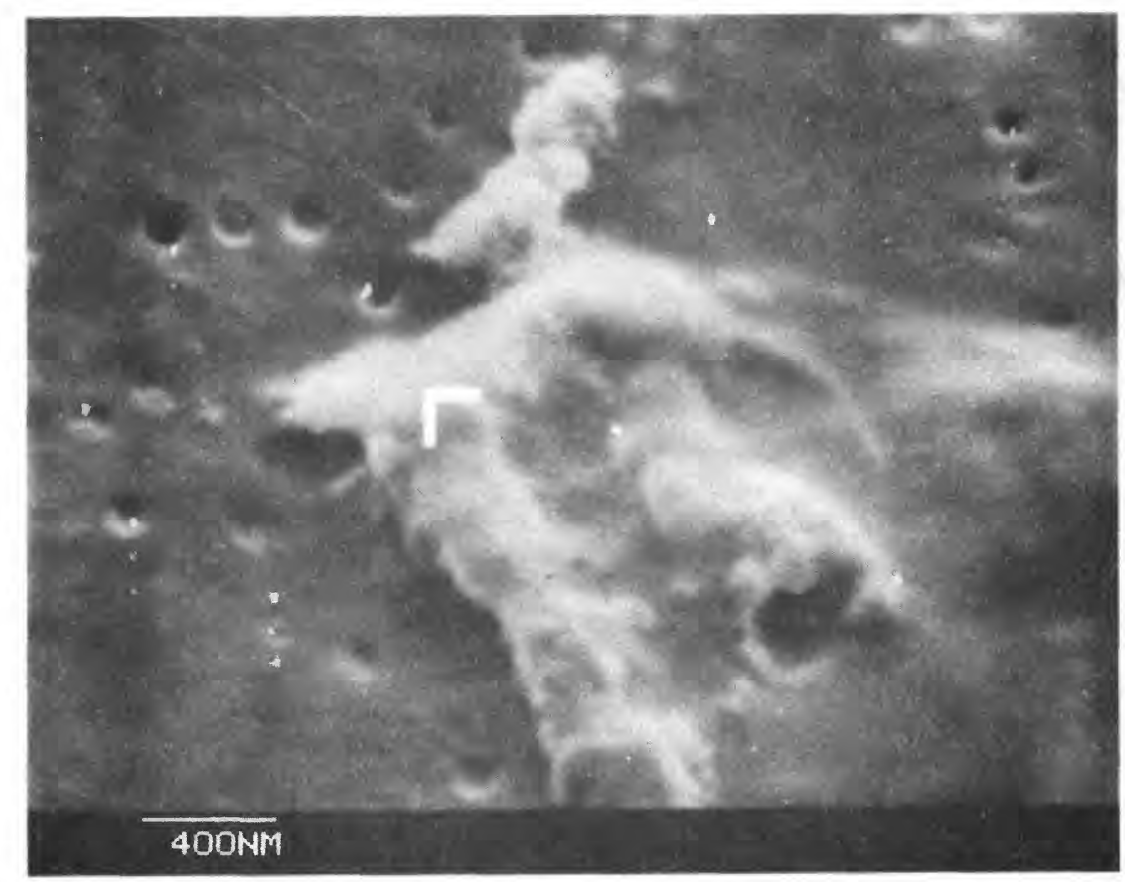

Figure 12A.--Scanning-electron photomicrograph of particle 08 retained on 100-NM filter, from water collected from the pool at the Sheeler seep, whitewood Creek, August 1986.

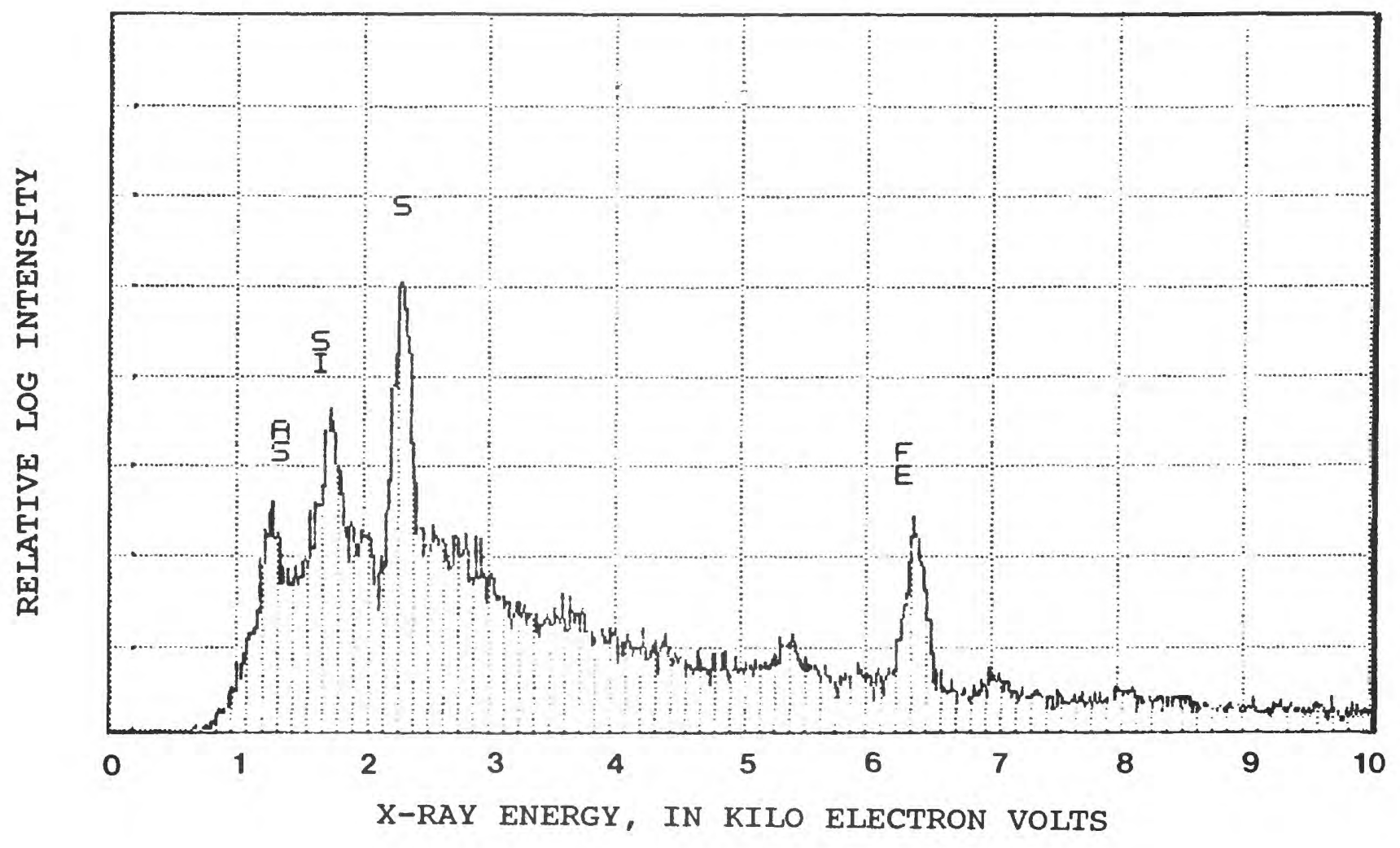

Figure 12B.--Energy dispersive X-ray analysis of particle 08 retained on 100-NM filter, from water collected from the pool at the Sheeler seep, Whitewood Creek, August 1986. 


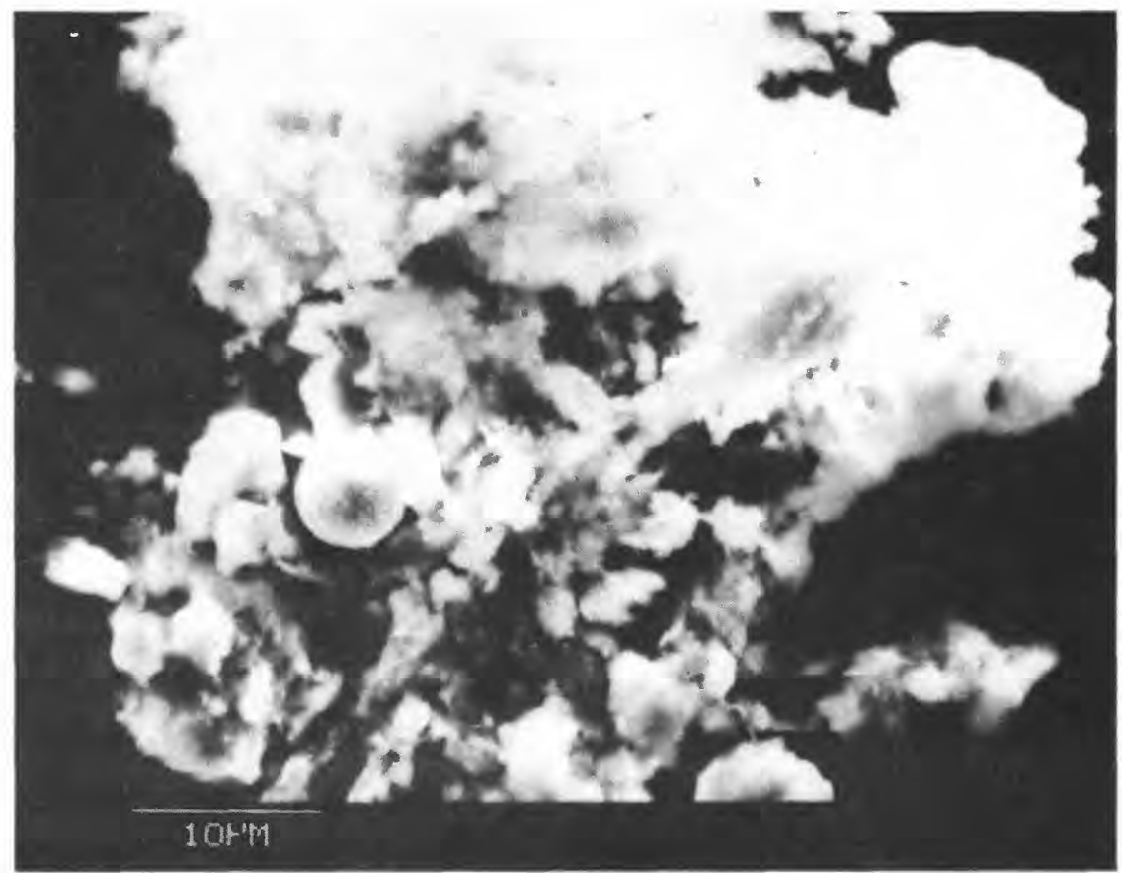

Figure 13A.--Scanning-electron photomicrograph of aggregate retained on 450-NM filter, from water collected from Whitewood creek below the Sheeler seep, August 1986.

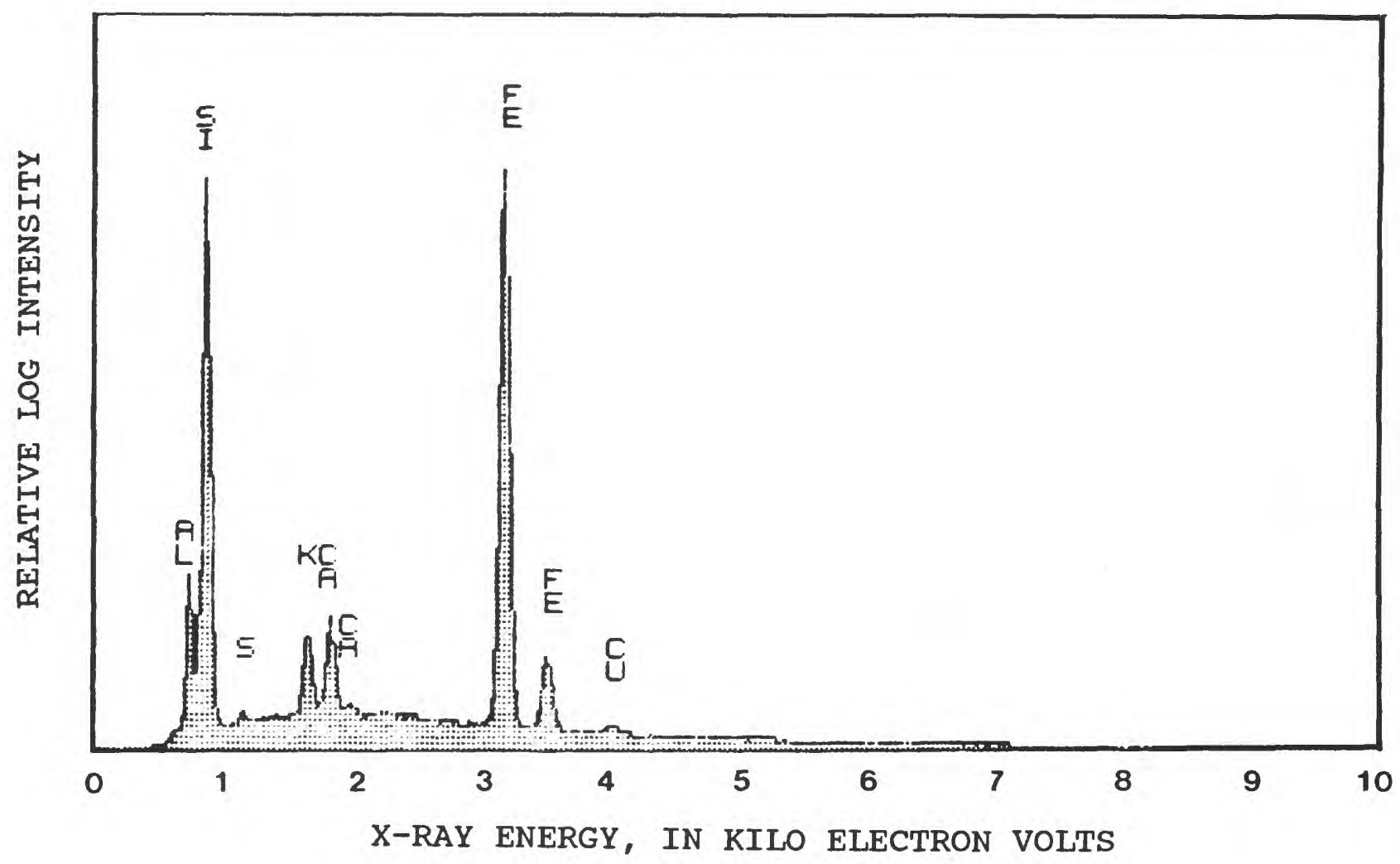

Figure 13B.--Energy dispersive X-ray analysis of aggregate retained on 450-NM filter, from water collected from Whitewood creek below the Sheeler seep, August 1986. 


\section{REFERENCES}

Fishman, M.J., and Friedman, L.C., eds., 1985, Methods for determination of inorganic substances in water and fluvial sediments: U.S. Geological Survey Open-File Report 85-495.

Garbarino, J.R., and Taylor, H.E., 1979, An inductively-coupled plasma atomicemission spectrometric method for routine water quality testing: Applied Spectroscopy, v. 33, p. 220-226.

Rees, T.F., 1987, A review of light scattering techniques for the study of colloids in natural waters: Journal of Contaminant Hydrology, $p$. 425-439. 


\section{Chapter I. Desorption of Arsenic from Iron Oxyhydroxide}

By Christopher C. Fuller, James A. Davis, and Regina G. Claypool-Frey

\section{SAMPLE COLLECTION AND HANDLING PROCEDURES}

A series of experiments were conducted to determine the potential for the desorption of arsenic associated with iron oxide precipitates that form where ground waters seep out of alluvial and tailings deposits at, or just above, stream level.

Samples of iron precipitate were collected at three ground-water discharge points adjacent to Whitewood Creek: the Berger site, Whitewood Creek above Vale, and the sheeler site (fig. 1, table 1). All apparatus and containers were acid cleaned prior to use in the field. Precipitate was collected out of pools that form below seep outlets and above stream level by drawing the floc (precipitate) of the bottom with a 50-mL syringe fitted with teflon tubing. Care was taken to minimize inclusion of coarser sediment below the precipitate. The floc was transferred into a $250-\mathrm{mL}$ polypropylene centrifuge bottle and allowed to settle. The supernatant was then decanted and the bottle refilled with more precipitate. This process was repeated several times until sufficient material was obtained. An additional precipitate sample was formed by collecting water from the seep at the Sheeler site and allowing oxidation and precipitation of the iron to occur upon exposure to the atmosphere. A 12-inch section of perforated one-half-inch inside diameter PVC tubing covered with 60- $\mu \mathrm{m}$ nylon screen was inserted horizontally into the bank at the seep outlet at the contact between the overlying alluvium and the Pierre Shale. The outlet of PVC pipe was fitted with a section of one-fourthinch outside diameter nylon tubing. Water was allowed to flow for 30 minutes before collection. During this period the outflow became clear. Flow was estimated at about $100 \mathrm{~mL}$ per minute. A 4-L polyethylene jar was then filled to overflowing with outlet of the tubing at the bottom of the jar. The sample was refrigerated and later purged with air to facilitate oxidation. An additional water sample was taken for chemical analyses by diverting flow into the top reservoir of a $\mathrm{N}_{2}$-purged filtration tower, filtered through a $0.4-\mu \mathrm{m}$ membrane filter, and preserved by acidification before exposure to the atmosphere. The sample was then processed in the same manner as ground water collected from piezometers (see Chapter G). A seep-water sample was collected at the Whitewood Creek above Vale site by syringe directly from the seep outlet and immediately filtered and processed as above. It was originally intended to collect a water sample at the Berger site; however, no sample was collected since ground-water input was from below the surface of a large pool adjacent to the stream. 


\title{
ANALYTICAL PROCEDURES AND RESULTS
}

\begin{abstract}
Water samples were processed, stored, and analyzed by the methods outlined in the ground-water geochemistry section of this report (Chapter G). Results are presented in table 47. Precipitates collected from pools below seeps were allowed to settle under refrigeration upon return to laboratory. Precipitate was then resuspended by shaking the decanted into another bottle after settling for 30 seconds. This process was repeated to ensure removal of coarser non-precipitate particulates. The supernatants were then decanted to produce a more concentrated slurry of about $50 \mathrm{~mL}$. The sample precipitated from the collected seep water was concentrated by the same method. Aliquots were transferred to tared vials, dried in a laminar flow hood at room temperature, weighed and corrected for salt weight, and dissolved in cold concentrated $\mathrm{HNO}_{3}$ for total iron and arsenic analyses. Iron was determined colorimetrically and arsenic by graphite furnace atomic absorption. Results are presented in table 48.
\end{abstract}

\section{DESORPTION EXPERIMENTS AND RESULTS}

Desorption of arsenic was studied as a function of time and phosphate concentration at $\mathrm{pH} 8.0$ in an artificial stream medium initially containing no arsenic. Desorption was initiated by adding aliquots of the precipitate $(1 \mathrm{~mL}$ ) into $30 \mathrm{~mL}$ of artificial stream water (ASW) in sets of $40 \mathrm{~mL}$ polycarbonate centrifuge tubes. Phosphate was added to additional sets of tubes as it has been shown to compete with arsenate for surface adsorption sites (Goldberg, 1986). Duplicate tubes were prepared for each sampling time and phosphate concentration. The resulting solids concentration ranged from 0.4 to $1.6 \mathrm{~g} / \mathrm{L}$ and was determined for each precipitate sample by the dry weights of three additional aliquots. The ASW was prepared from reagent-grade salts to yield similar major ion composition to Whitewood Creek. The $\mathrm{pH}$ of the ASW was adjusted to 8.0 by addition of $\mathrm{NaHCO}_{3}$ and $\mathrm{Na}_{2} \mathrm{CO}_{3}$ to yield the total dissolved $\mathrm{CO}_{2}$ and $\mathrm{pH}$ desired (composition in table 49). Phosphate was added during the preparation to yield 10 or $100 \mu \mathrm{M}$ concentration. Upon addition of the iron precipitate slurry to the ASW, tubes were mixed by rotating end over end at $30 \mathrm{rpm}$. After the desired reaction period, the $\mathrm{pH}$ was measured on pairs of tubes for each precipitate sample and phosphate concentration. Tubes were then centrifuged at $10,000 \mathrm{~g}$ for 10 minutes and the supernatant was sampled by pipetting. The arsenic concentration in the supernatant was determined by hydride generation atomic absorption following reduction with $K I$. Results are presented in tables 50 through 53 along with corresponding streamwater arsenic concentrations and the predicted equilibrium arsenate concentration ( $C_{\text {e }}$ ) from an adsorption isotherm at $\mathrm{pH} 8$ for the arsenateamorphous iron oxide system (Pierce and Moore, 1982).

\section{REFERENCES}

Goldberg, S., 1986, Chemical modeling of arsenate adsorption on aluminum and iron oxide minerals: Soil Science Society of America Journal, v. 50, p. 1154 .

Pierce, M.L., and Moore, C.B., 1981, Adsorption of arsenite and arsenate on amorphous iron hydroxide: Water Research, v. 6, p. 1247. 


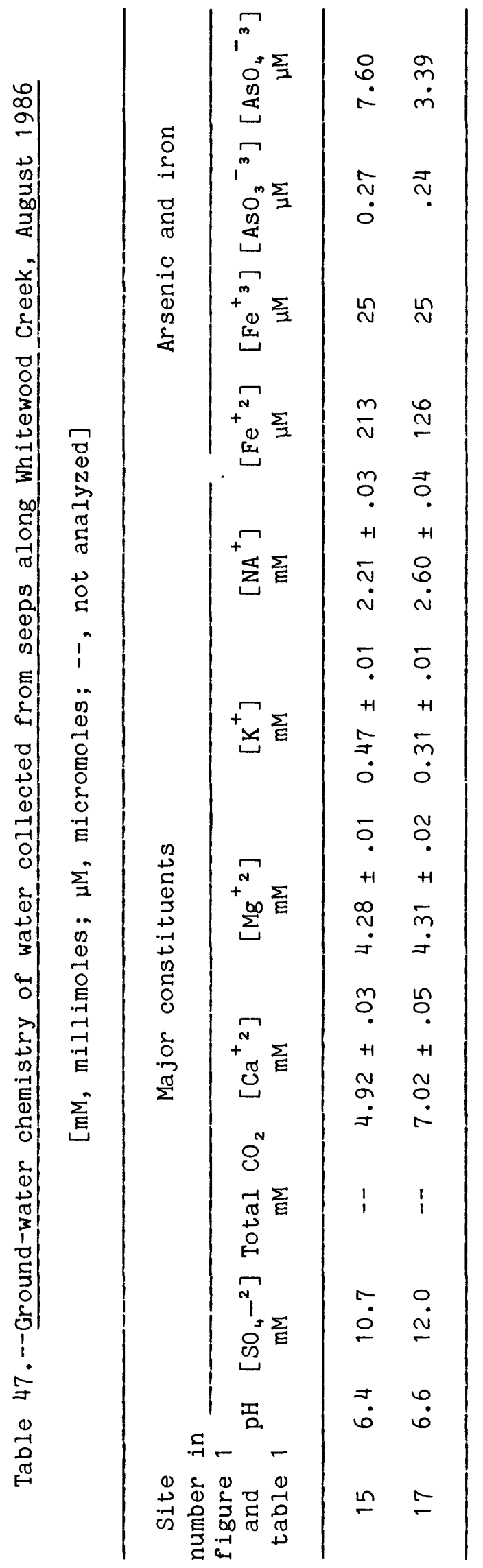


Table 48.--Arsenic and iron content of iron oxyhydroxide precipitates collected from seeps adjacent to whi tewood Creek, August 1986

[mM/g, millimoles per gram]

Site number

in figure 1 and table 1

Arsenic

$(\mathrm{mM} / \mathrm{g})$
Iron

$(\mathrm{mM} / \mathrm{g})$

Ratio

$\begin{array}{llll}13 & 0.0809 \pm .0050 & 3.133 \pm .167 & 0.0258 \pm .0021 \\ 15 & 0.1092 \pm .0067 & .274 \pm .73 & 0.0207 \pm .0031 \\ 17^{1} & 0.0505 \pm .0012^{2} & 5.014 \pm .101^{2} & 0.0101 \pm .0003 \\ 17^{3} & 0.0236 \pm .0023 & 1.765 \pm .011 & 0.0134 \pm .0013\end{array}$

${ }^{1}$ Natural precipitate.

${ }^{2}$ Standard deviation of three dissolutions and analyses.

${ }^{3}$ Artificial precipitate.

Table 49.--Artificial stream-water composition

Species

Concentration

(millimoles)

$\begin{array}{lc}\mathrm{SO}_{4}{ }^{-2} & 3.5 \\ \mathrm{Cl}^{-} & .7 \\ \mathrm{Total} \mathrm{CO}_{2} & 2.0 \\ \mathrm{Ca}^{2}{ }^{+} & 1.9 \\ \mathrm{Mg}^{2}{ }^{+} & 1.9 \\ \mathrm{~K}^{+} & .29 \\ \mathrm{Na}^{+} & 1.9\end{array}$


Table 50.--Desorption of arsenic from iron oxide precipitates from the seep adjacent to Whitewood Creek at the Berger site; as a function of time and phosphate concentration

[Precipitate collected in pool below inactive seep outlet]

As $: F e=0.026$ solid concentration $=0.7$ grams per liter

Predicted $\mathrm{C}_{\mathrm{eq}}=10 \mu \mathrm{M}$ (micromoles)

Measured [As] in stream $=0.94 \pm .04 \mu \mathrm{M}$

\begin{tabular}{|c|c|c|c|}
\hline $\begin{array}{l}\text { Time } \\
\text { (hours) }\end{array}$ & $\begin{array}{c}{\left[\mathrm{PO}_{4}{ }^{-}\right]} \\
(\mu \mathrm{M})\end{array}$ & $\begin{array}{c}\mathrm{pH} \\
(\mathrm{f} \text { inal })\end{array}$ & $\begin{array}{l}{[\mathrm{As}]} \\
(\mu \mathrm{M})\end{array}$ \\
\hline 1 & 0 & 8.16 & 0.31 \\
\hline 24 & 0 & 8.17 & .31 \\
\hline 144 & 0 & 8.06 & .31 \\
\hline 1 & 10 & 8.25 & .61 \\
\hline 24 & 10 & 8.19 & .49 \\
\hline 144 & 10 & 8.09 & .49 \\
\hline 288 & 10 & 8.04 & .48 \\
\hline 1 & 100 & 8.25 & 2.9 \\
\hline 24 & 100 & 8.12 & 3.3 \\
\hline 144 & 100 & 8.12 & 3.1 \\
\hline 288 & 100 & 8.01 & 2.9 \\
\hline
\end{tabular}

\% As desorbed: $0.4 \%$ to $4.1 \%$. 
Table 51.--Desorption of arsenic from iron oxide precipitates from the seep adjarent to Whitewood Creek above Vale; as

a function of time and phosphate concentration

[Precipitate collected of rock below seep outlet, above stream]

As: $\mathrm{Fe}=0.021$ solid concentration $=1.5$ grams per liter

Predicted $\mathrm{C}_{\text {eq }}=8 \mu \mathrm{M}$ (micromoles)

Measured [As] in stream $=0.60 \pm .04 \mu \mathrm{M}$

\begin{tabular}{rccc}
$\begin{array}{c}\text { Time } \\
\text { (hours) }\end{array}$ & $\begin{array}{c}{\left[\mathrm{PO}_{4}{ }^{-}\right]} \\
(\mu \mathrm{M})\end{array}$ & $\begin{array}{c}\mathrm{pH} \\
(\mathrm{final})\end{array}$ & $\begin{array}{c}{[\mathrm{As}]} \\
(\mu \mathrm{M})\end{array}$ \\
\hline 1 & 0 & 8.01 & 0.11 \\
24 & 0 & 7.84 & .05 \\
144 & 0 & 7.81 & .07 \\
1 & 10 & 7.95 & .11 \\
24 & 10 & 7.90 & .09 \\
144 & 10 & 7.75 & .08 \\
11 & & 7.99 & .13 \\
144 & 100 & 7.82 & .08 \\
\hline
\end{tabular}

$\%$ As desorbed: $0.3 \%$ to $2.5 \%$. 
Table 52.--Desorption of arsenic from iron oxide precipitates collected from the seep adjacent to whitewood Creek at the Sheeler site; as a function of time and phosphate concentration

[Precipitate collected of rock below seep outlet, above stream]

As $: F e=0.010$ solid concentration $=1.6$ grams per liter

Predicted $\mathrm{C}_{\mathrm{eq}}=0.7 \mu \mathrm{M}$ (micromoles)

Measured [As] in stream $=0.52 \pm .01 \mu \mathrm{M}$

\begin{tabular}{rrrr}
$\begin{array}{c}\text { Time } \\
\text { (hours) }\end{array}$ & $\begin{array}{c}{\left[\mathrm{PO}^{3}{ }^{-}\right]} \\
(\mu \mathrm{M})\end{array}$ & $\begin{array}{c}\mathrm{pH} \\
(\mathrm{final})\end{array}$ & $\begin{array}{c}{[\mathrm{As}]} \\
(\mu \mathrm{M})\end{array}$ \\
\hline 1 & 0 & 8.02 & $<0.01$ \\
24 & 0 & 7.87 & $<.01$ \\
144 & 0 & 7.95 & .03 \\
1 & 10 & 8.00 & .02 \\
24 & 10 & 7.92 & .01 \\
144 & 10 & 7.87 & .03 \\
1 & 100 & 8.01 & .04 \\
1 & 100 & 7.91 & .03 \\
\hline
\end{tabular}

$\%$ As desorbed: $0.01 \%$ to $0.05 \%$. 
Table 53.--Desorption of arsenic from iron oxide precipitates formed from water from the seep adjacent to whitewood Creek at the Sheeler site; as a function of time and phosphate concentration

[Precipitate formed in collected seep water]

As $: \mathrm{Fe}=0.013$ solid concentration $=0.4$ grams per liter

Predicted $\mathrm{C}_{\mathrm{eq}}=1.3 \mu \mathrm{M}$ (micromoles)

Measured [As] in stream $=0.52 \pm .01 \mu \mathrm{M}$

\begin{tabular}{rccc}
$\begin{array}{c}\text { Time } \\
\text { (hours) }\end{array}$ & $\begin{array}{c}{\left[\mathrm{PO}^{3}{ }^{-}\right]} \\
(\mu \mathrm{M})\end{array}$ & $\begin{array}{c}\mathrm{pH} \\
(\mathrm{final})\end{array}$ & $\begin{array}{c}{[\mathrm{As}]} \\
(\mu \mathrm{M})\end{array}$ \\
\hline 1 & 0 & 8.19 & 0.04 \\
144 & 0 & 7.96 & .07 \\
14 & 0 & 8.18 & .05 \\
24 & 10 & & .20 \\
144 & 10 & 8.30 & .11 \\
288 & 10 & 8.06 & .11 \\
1 & 10 & 8.27 & .11 \\
24 & 100 & 8.03 & .33 \\
144 & 100 & 8.31 & .73 \\
288 & 100 & 8.12 & 1.09 \\
& 100 & 8.30 & 1.26 \\
\hline
\end{tabular}

\% As desorbed: $0.4 \%$ to $13 \%$. 


\title{
Chapter J. Blological Investigations - Surface-Water Toxics Program, Whitewood Creek and the Belle Fourche and Cheyenne Rivers, South Dakota
}

\author{
By James S. Kuwabara, Daniel J. Cain, James L. Carter, and Steven V. Fend
}

\section{INTRODUCTION}

Biological processes are important components of water-quality studies. Currently there are two National Research Program projects addressing different aspects of these processes in relation to the surface-water toxics studies being conducted in the Cheyenne River basin of western South Dakota. One project is studying the influence of stream algal populations on arsenic (As) transport. The other project is using stream benthic insects as bioindicators to delineate the extent of As transport in Whitewood Creek and the Belle Fourche and Cheyenne Rivers. The following section will present data from the initial sampling trips of these projects. A subsection describing periphyton studies will be presented first followed by another on the benthic insect work. In each subsection, the study objectives and sampling and analytical methods are discussed prior to the presentation of tabulated data. A brief description of future experiments also is given. Some interpretation of the initial biological data was presented as extended abstracts (Cain and others, 1988; Kuwabara and others, 1988).

\section{WORK ON PERIPHYTON}

It has been inferred in recent research that elevated arsenate concentrations inhibit primary production by interfering with phosphate metabolism (Button and others, 1973; Sanders, 1979). Algal metabolism may in turn affect the chemical speciation of arsenic in natural waters by formation and release of methylated arsenicals (Andreae, 1977) and by decomposition of senescent cellular material. The interdependence of algal growth and the transport and speciation of As in Whitewood Creek is being investigated. The creek represents an ideal site for such a study because: (1) Benthic macrophytes and algae are visibly abundant during the summer months, and (2) municipal sewage discharge into the creek, as well as As-enriched mine tailings deposited along the 25-mi study reach, generate dissolved phosphate and arsenate concentration gradients. Thus, Whitewood creek is a logical site to examine phosphate-aresenate interactive effects. Arsenic transport parameters are being determined for isolates of a chlorophyte, Stichococcus $\mathrm{sp}$. , common to the four collection sites along Whitewood Creek from upstream of the discharge from a mine to a site upstream from the confluence of Whitewood Creek and the Belle Fourche River. These parameters (that is, algal standing crop, sorption and release rates, and accumulation factors) may be used in a biological component of a comprehensive transport model comprised of hydrologic, chemical, and biological terms.

The initial, and thus far only, sampling trip to whitewood Creek occurred during August 4-9, 1986. Algal standing crop (periphyton abundance) was estimated from cobble scrappings $(n=6)$ taken from four sites: Whitewood Creek above Lead (site 1), Whitewood Creek below Deadwood STP (site 8), Whitewood Creek above Vale (site 15), and Whitewood Creek at the Sheeler site (site 17) (fig. 1, table 1). Acetone extractions of chlorophyll-a were analyzed 
fluorometrically (Franson, 1985). Ash-free dry weights were also determined from cobble scrappings in order to calculate an autotrophic index (mass ratio of periphyton to chlorophyll-a) for each site as a potential indicator of environmental stress. Estimates for periphyton abundance (table 54) represent the ash-free dry weights per unit area of scrappings multiplied by the percent coverage of algal growth at the sampling site.

Table 54.--Estimated periphyton standing crop ( $P$ in grams per square meter) for four sites along Whitewood Creek sampled in August 1986

[The mass ratio of periphyton to chlorophyll-a (autotrophic index) has been used as an indicator of environmental stress with increasing values indicating greater abundance of senescent algal cells or increased heterotrophic growth (Weber, 1973)]

\begin{tabular}{cccc}
$\begin{array}{c}\text { Site } \\
\text { figure 1 } \\
\text { and table 1) }\end{array}$ & $\begin{array}{c}\text { Temperature } \\
\left({ }^{\circ} \mathrm{C}\right)\end{array}$ & $\begin{array}{c}\text { Autotrophic } \\
\text { index }\end{array}$ & $\begin{array}{c}\mathrm{P}_{\mathrm{b}} \text { (grams per } \\
\text { square meter) }\end{array}$ \\
\hline 1 & 13.2 & 120 & $21 \pm 14$ \\
8 & 17.5 & 210 & $34 \pm 13$ \\
15 & 19.0 & 140 & $77 \pm 27$ \\
17 & 24.2 & 190 & $25 \pm 11$ \\
\hline
\end{tabular}

Algal species isolation procedures were conducted to obtain a species common to all four sampling sites for subsequent use in As and $P$ sorption studies. Algal suspensions generated from periphyton scrappings were streaked on 1-percent agar plates containing an algal growth medium (Kuwabara and others, 1985) enriched with silica. Algal clusters that formed on the plates were transferred to liquid media for one week to increase algal density. This agar plate streaking and resuspension procedure was repeated until unialgal cultures were achieved. Two isolates were obtained that were common to the four sampling sites. Both were chlorophytes: Stichococcus sp. and Scenedesmus sp. It is not clear why common diatoms were not obtained from this isolation procedure, because an abundance of diatoms was observed in the initial scrappings. One possibility is that chlorophyte growth was more rapid than diatoms on these agar plates. Another possibility is that the agar color obscured diatom clusters on the plates while chlorophyte clusters were readily visible. Stichococcus isolates were selected over scenedesmus for use in subsequent sorption experiments, because in agitated cultures, Stichococcus remained unicellular and could therefore be quickly and precisely enumerated with a particle counter. Algal isolates were maintained in a chemically defined growth medium with added arsenate and orthophosphate concentrations similar to ambient levels at their respective collection sites. 
In initial sorption experiments, uptake of arsenate and phosphate were observed in living and heat-killed cells from three collection sites (Whitewood Creek above Lead, Whitewood Creek above Deadwood STP, and Whitewood Creek above Vale). Algal suspensions, in $40 \mathrm{~mL}$ fluoroethylene polymer Oakridge tubes, were spiked with ${ }^{73} \mathrm{As}$ and ${ }^{32} \mathrm{P}-l$ labelled stock solutions yielding a $0.5 \mu \mathrm{M}$ concentration for both total dissolved As and $\mathrm{P}$. Suspensions were then placed on a mechanical shaker and agitated. After 24 and 48 hours of exposure, suspensions were centrifuged at $20,000 \mathrm{rpm}$ for 10 minutes to remove the algal cells from suspension. Five-milliliter volumes of the resulting supernatant, the algal suspension before centrifugation, and an internal ${ }^{7}{ }^{3} \mathrm{As}$ and ${ }^{32} \mathrm{P}$ standard were counted by liquid scintillation to determine the extent of As and orthophosphate uptake by these cultures (table 55).

Table 55.--Algal uptake at 24 and 48 hours of arsenate and or thophosphate by living and heat-killed cells using 3 stichococcus isolates from sites on Whitewood Creek (August 1986)

[The calculated 95-percent confidence intervals are given for 3 replicate cultures. Culture media additions of total As and total $\mathrm{P}$ at $0.5 \mu \mathrm{M}$ were used]

\begin{tabular}{|c|c|c|c|c|c|}
\hline \multirow{2}{*}{$\begin{array}{c}\text { Site } \\
\text { (figure 1 } \\
\text { and table 1) }\end{array}$} & \multirow[b]{2}{*}{$\begin{array}{l}\text { Time } \\
\text { (hours) }\end{array}$} & \multicolumn{2}{|c|}{$\begin{array}{l}\text { Percent } \\
\text { As sorbed }\end{array}$} & \multicolumn{2}{|c|}{$\begin{array}{l}\text { Percent } \\
P \text { sorbed }\end{array}$} \\
\hline & & Living & $\begin{array}{l}\text { Heat- } \\
\text { killed }\end{array}$ & Living & $\begin{array}{l}\text { Heat- } \\
\text { killed }\end{array}$ \\
\hline 1 & $\begin{array}{l}24 \\
48\end{array}$ & $\begin{array}{l}25 \pm 12 \\
23 \pm 8\end{array}$ & $\begin{array}{l}29 \pm 11 \\
20 \pm 6\end{array}$ & $\begin{array}{l}93 \pm 2 \\
96 \pm 2\end{array}$ & $\begin{array}{l}94 \pm 2 \\
95 \pm 2\end{array}$ \\
\hline 8 & $\begin{array}{l}24 \\
48\end{array}$ & $\begin{array}{l}19 \pm 3 \\
20 \pm 4\end{array}$ & $\begin{array}{l}27 \pm 10 \\
18 \pm 6\end{array}$ & $\begin{array}{l}93 \pm 2 \\
96 \pm 2\end{array}$ & $\begin{array}{l}90 \pm 4 \\
92 \pm 2\end{array}$ \\
\hline 15 & $\begin{array}{l}24 \\
48\end{array}$ & $\begin{array}{l}24 \pm 6 \\
16 \pm 5\end{array}$ & $\begin{array}{l}21 \pm 10 \\
19 \pm 3\end{array}$ & $\begin{array}{l}88 \pm 2 \\
94 \pm 2\end{array}$ & $\begin{array}{l}84 \pm 2 \\
84 \pm 2\end{array}$ \\
\hline
\end{tabular}

Uptake rates of arsenate and orthophosphate by algal isolates are currently being studied as a function of added concentrations of dissolved As and $P$. A method to determine As speciation by high performance liquid chromatography coupled with hydride generation atomic absorption spectroscopy is currently being developed by Ann Maest and Sofie Pasilis of the National Research Program in Menlo Park, California. This method will be used to determine uptake and release rates for methylated as well as inorganic forms of As for algal isolates from the four sampling sites. Periphyton will be sampled on a monthly basis and taxonomically analyzed during future studies to gain a better understanding of the dynamics of the periphyton communities along the study reach. 


\section{ARSENIC IN BENTHIC INSECTS}

There are numerous methods that use aquatic organisms in the analysis of water quality. The presence, absence, and/or relative abundance of known sensitive species has been used to delineate impacted areas (Warren, 1977), while the analysis of community composition has been shown to be a sensitive measure of the effects of toxic contaminants on lotic ecosystems (Leland and others, 1984). The determination of contaminants in the tissues of aquatic organisms can be used to define areas of pollution (Bryan and others, 1985; Goldberg and others, 1978; Thomson and others, 1984). This later method can provide information on the downstream transport of a contaminant in a river system and the penetration of that contaminant into aquatic food webs.

Benthic insects were collected with kick nets ( 3 to 5 minutes of kicking per sample) at 10 locations in Whitewood Creek and the Belle Fourche and Cheyenne Rivers in June and August 1986 and preserved in ethanol for the analysis of community structure. Additional samples were taken at 6 of these sites for elemental analysis. Two stations were located outside the contaminated area. The insects were kept in cooled stream water for at least 6 hours to clear their digestive tract and then were frozen using dry ice. In the laboratory, the samples were thawed, thoroughly rinsed with de-ionized water, and sorted by taxonomic grouping and individual size. Individuals were pooled to attain enough biomass (ca. $100 \mathrm{mg}$ dry weight) for elemental analysis. Total As was determined by modified procedures of May (1982) and Penrose and others (1975). Samples were homogenized with a Polytron highspeed blender and then lyophilized. One-hundred to $200 \mathrm{mg}$ of the lyophilized material was wetted with methanol in a 125-mL erlynmeyer flask. Five milliliters of $2.7 \mathrm{M} \mathrm{Mg}\left(\mathrm{NO}_{3}\right)_{2}$, an ashing aid, and $0.5 \mathrm{~mL}$ of antifoaming emulsion (Dow Corning DB-110A) was added to the sample. The mixture was dried overnight at $80^{\circ} \mathrm{C}$. The samples were removed from the oven, covered, and then dry-ashed in a muffle furnace at $450^{\circ} \mathrm{C}$ overnight. The temperature was increased in increments of approximately $75{ }^{\circ} \mathrm{C}$ per hour. The ashed sample was dissolved in $25 \mathrm{~mL}$ of 50 percent HCL. Five milliliters of this solution was transferred to a glass vial and pre-reduced with $0.5 \mathrm{~mL}$ of 50 percent $\mathrm{KI}$ solution ( 1 part KI:10 parts sample) 1 hour before the analysis. Arsenic was determined by hydride generation atomic absorption spectroscopy (AAS) with a Perkin-Elmer MHS-10. A 30-percent HCL solution was used for the diluent. The analytical accuracy was checked by analyzing U.S. National Bureau of Standards standard reference material (SRM 50) and by determining the recovery of As in samples spiked with As (as $\mathrm{NaAsO}_{2}$ ). Subsamples of the ashed material also were acidified with 50 percent HCL and analyzed for Fe by flame AAS. Samples were corrected for blanks.

Recovery of As in As-spiked samples ranged between 90 and 108 percent. The As concentration determined for SRM 50 ( $3.23 \pm 0.24 \mu \mathrm{g} \mathrm{As} / \mathrm{g}$ dry weight) agreed with the recommended value $(3.3 \pm 0.4 \mu \mathrm{g} / \mathrm{g})$. 
Species abundance of samples collected in June and August 1986 is shown in table 56. The composition and abundance of species displayed considerable variation among sites and between months. The upstream portions of Whitewood Creek were dominated by cold water species (for example, Rhyacophila sp., Hesperoperla pacifica, and Ephemerella inermis) which were generaliy more abundant in June than in August. In contrast, warm water or silt-tolerent species (for example, Choroterpes sp., Tricorythodes sp., and Dactylobaetis sp.) were more abundant in the downstream areas in june than in August.

Arsenic concentrations in insects at Whitewood Creek below Gold Run (site 5) was 5 to 6 times greater than background concentrations at Whitewood Creek above Lead (site 1) (table 57). The highest concentrations were at Whitewood Creek above Whitewood (site 10). Arsenic concentration also varied greatly among species within a site.

Iron concentrations were more variable and did not display as strong a downstream gradient as As (table 58).

Future research will compare As concentrations in insects against As concentrations of sediments and in solution. In order to eliminate problems relating to the limited geographical distribution of individual species, we will examine different sampling techniques, and evaluate the effect of grouping organisms at taxonomic levels higher than species. The influence of trophic level as a source of the interspecific variation in As concentration also will be studied. Longer-term goals are to study the mechanisms of As uptake by benthic insects and the effects of benthic insect grazing on the uptake of As by algae. 
Table 56.--Relative abundance in 3-5 minute kick samples of selected benthic insects in whitewood Creek, Belle Fourche River, and the Cheyenne River

[Relative abundance ${ }^{1}$ of selected taxa, June 1986]

Site number ${ }^{2}$

$\begin{array}{llllllllll}1 & 5 & 8 & 10 & 13 & 17 & 19 & 20 & 26 & 35\end{array}$

Baetid Mayflies

Baetis tricaudatus

Baetis brunneicolor

Baetis flavigistra

Baetis nr. pygmaeus

Baetis sp.

Dactylobaetis sp.

Paracioeodes minutus

Pseudocloeon sp.

$\begin{array}{lllllllll}6 & 6 & & & 6 & 4 & & & \\ 4 & 3 & 5 & 1 & & & & & \\ & 2 & 6 & 2 & & 1 & & & \\ & & & & & & 1 & 1 & 1 \\ & & & & & 1 & 3 & 4 & \\ & & & & & 1 & 2 & 4 & \\ & & 1 & 5 & 6 & & 3 & 2 & 1\end{array}$

Miscellaneous Taxa

Ephemerella inermis $\quad \begin{array}{lllll}4 & 4 & 1 & 2\end{array}$

Choroterpes sp.

Tricorythodes sp.

Leucrocuta sp.

Hesperoperla pacifica

Rhyacophila sp.

Limnephilus sp.

$\begin{array}{llll}4 & 4 & 1\end{array}$

Hydropsychid Caddisflies

Hydropsyche oslari ? 2

Hydropsyche slossonae 1

Hydropsyche bronta?

Cheumatopsyche sp. 1

Cheumatopsyche sp. 2

$\begin{array}{lllll}3 & 4 & 1 & 4 & 3 \\ 4 & 3 & 1 & 1 & 1 \\ 1 & 2 & 2 & 5 & 1\end{array}$

2

11


Table 56.--Relative abundance in 3-5 minute kick samples of selected benthic insects in Whitewood Creek, Belle Fourche River, and the Cheyenne River--Continued

\begin{tabular}{|c|c|c|c|c|c|c|}
\hline & \multicolumn{6}{|c|}{ Site number 2} \\
\hline & 1 & 8 & 13 & 17 & 20 & 26 \\
\hline \multicolumn{7}{|l|}{ Baetid Mayflies } \\
\hline Baetis tricaudatus & 4 & 6 & & & & \\
\hline Baetis brunneicolor ? & 3 & & 4 & 3 & 5 & 4 \\
\hline$\overline{\text { Baetis }} \overline{\text { flavigistra }}$ & & & & & 4 & \\
\hline Dactylobaetis sp. & & & 1 & 4 & 4 & 3 \\
\hline Paracloeodes minutus & & & & & & 1 \\
\hline Pseudocloeon sp. & & 1 & 3 & 5 & 5 & 1 \\
\hline \multicolumn{7}{|l|}{ Miscellaneous Taxa } \\
\hline Choroterpes sp. & & & 5 & 4 & 5 & 5 \\
\hline Tricorythodes sp. & & & 5 & 4 & 4 & 1 \\
\hline Isonychia sp. & & & & 1 & 3 & 1 \\
\hline Ephoron album & & & 5 & 2 & $\hat{1}$ & 2 \\
\hline Limnephilus sp. & 1 & 1 & & & & \\
\hline \multicolumn{7}{|l|}{ Hydropsychid Caddisflies } \\
\hline Hydropsyche slossonae & 1 & 6 & & & & \\
\hline Hydropsyche bronta ? & & 4 & 4 & 5 & 6 & 1 \\
\hline Cheumatopsyche sp. 1 & & & & 4 & 6 & 3 \\
\hline Cheumatopsyche sp. 2 & & & 3 & 3 & 6 & 1 \\
\hline \multicolumn{7}{|l|}{${ }^{1}$ Abundance categories: } \\
\hline $1-0-5$ & & 4 & $20-50$ & & & \\
\hline $2-5-10$ & & 5 & $-50-100$ & & & \\
\hline $3-10-20$ & & 6 & $->100$ & & & \\
\hline
\end{tabular}




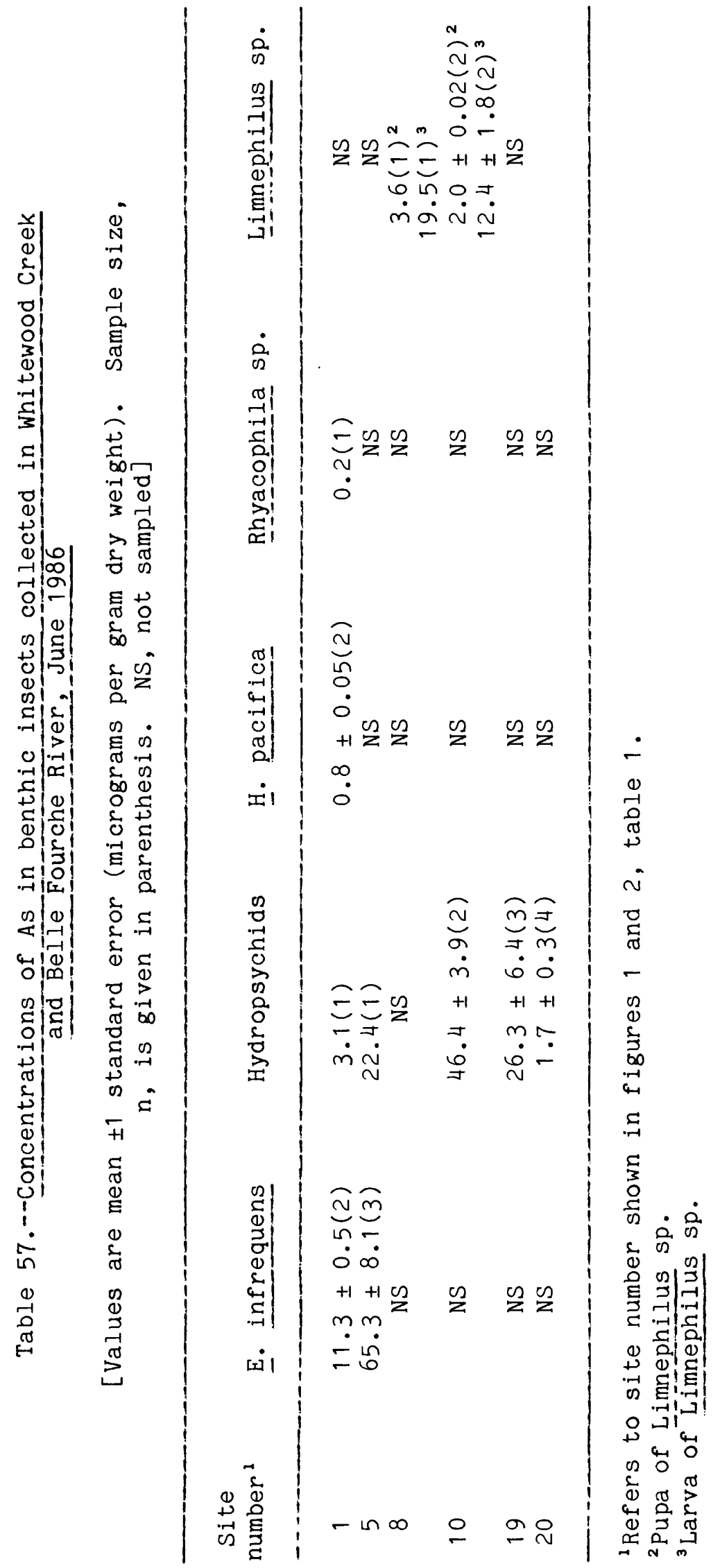




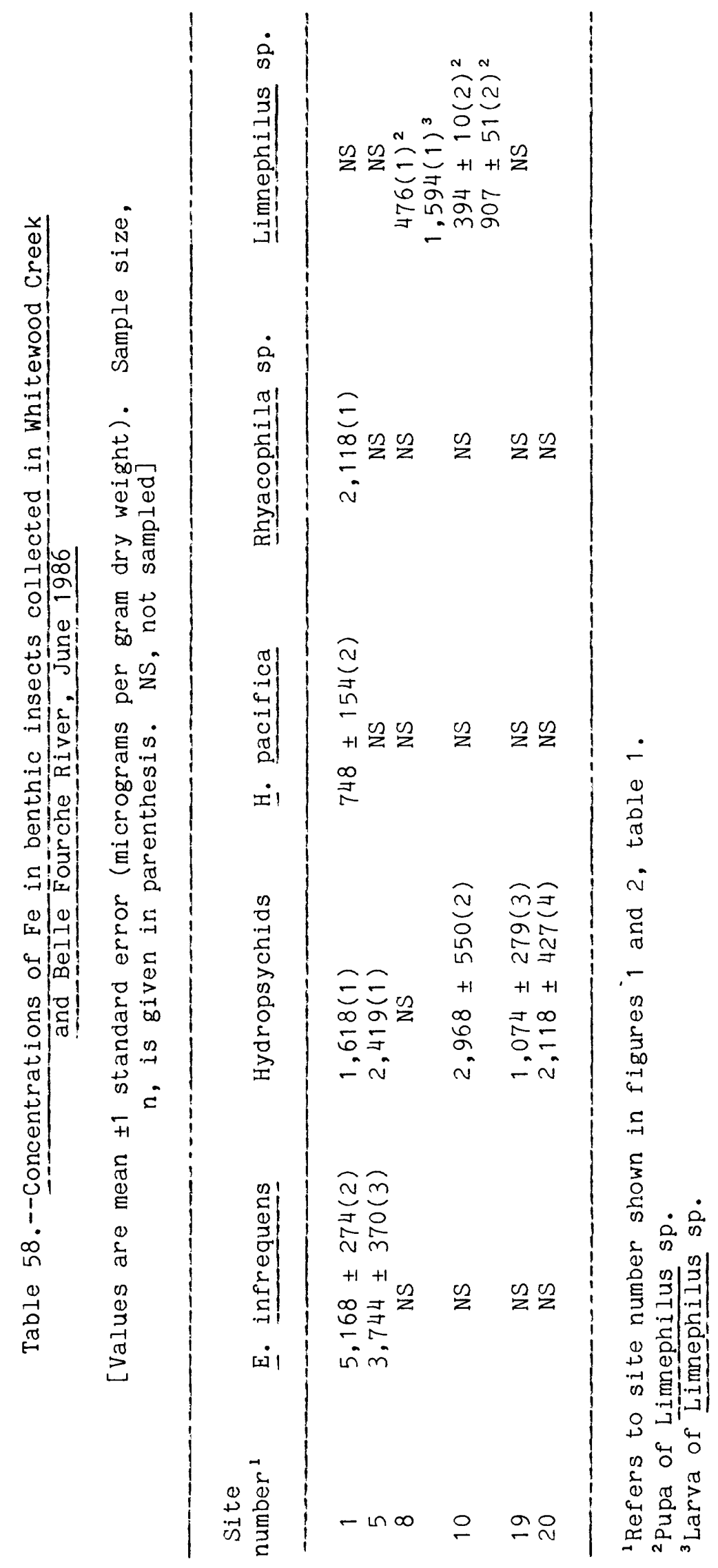




\section{SELECTED REFERENCES}

Bryan, G.W., Langston, W.L., Hummerstone, L.G., and Burt, G.R., 1985, A guide to the assessment of heavy-metal contamination in estuaries using biological indicators: Occasional Publication No. 4 of the Marine Biological Association of the United Kingdom, $92 \mathrm{p}$.

Cain, D., Carter, S., and Fend, S., 1988, Annual variation in arsenic concentration of benthic insects in Whitewood Creek South Dakota: Fourth Toxic Substances Hydrology Technical Meeting, Phoenix, Arizona, Proceedings, p. 55-60.

Franson, M.A.H., 1985, Standard methods for the examination of water and wastewater: 16th ed., American Public Health Association, American Water Works Association and Water Pollution Control Federation, 1,268 p.

Goldberg, E.D., Bowen, V.T., Farrington, J.W., Harvey, G., Martin, J.H., Parker, P.L., Risebrough, R.W., Robertson, W., Schneider, E., and Gumble, E., 1978, The mussel watch: Environmental Conservation, v. 5, p. 101-125.

Jorgensen, S.E., 1983, Modeling the ecological processes, in Orlob, G.T. ed., Mathematical modeling of water quality: Streams, lakes and reservoirs: John Wiley and Sons, Chichester, p. 116-149.

Kuwabara, J.C., Change, C.C.V., and Pasilis, S.P., 1988, Periphyton effects on arsenic transport in Whitewood Creek, South Dakota: Fourth Toxic Substances Hydrology Technical Meeting, Phoenix, Arizona, Proceedings, p. 33-37.

Kuwabara, J.S., Davis, J.A., and Chang, C.C.Y., 1985, Culturing Selenastrum capricornutum (Chlorophyta) in a synthetic algal nutrient medium with defined mineral particulates: Hydrobiologia, v. 122, p. 23-27.

Kuwabara, J.S., Leland, H.V., and Bencala, K.E., 1984, Copper transport along a Sierra Nevada stream: Journal of Environmental Engineering, v. 110, p. 646-655.

Leland, H.V., and Carter, J.L., 1984, Effects of copper on species composition of periphyton in a Sierra Nevada, California stream: Freshwater Biology, v. 14, p. 281-296.

May, T.S., 1982, Recovery of endogenous selenium from fish tissues by open system dry ashing: J. Assoc. Off. Anal. Chem., v. 65, p. 1140-1145.

Penrose, W.R., Black, R., and Hayward, M.J., 1975, Limited arsenic dispersion in sea water, sediments, and biota near a continuous source: J. Fish. Res. Board Can., v. 32, p. 1275-1281.

Phillips, D.J.H., 1980, Quantitative aquatic biological indicators: Applied Science Publishers Ltd., London, $488 \mathrm{p}$. 
Thomson, E.A., Luoma, S.N., Johansson, C.E., and Cain, D.J., 1984, Comparison of sediments and organisms in identifying sources of biologically available trace metal contamination: Water Res., v. 18, p. 755-765.

Weber, C.I., 1973, Biological field and laboratory methods for measuring quality of surface waters and effluents: U.S. Environmental Protection Agency, 670/4-73-001.

Zison, S.W., Mills, W.B., Deimer, D., and Chen, C.W., 1978, Rates, constants and kinetics formulations in surface water quality modeling: U.S. Environmental Protection Agency, EPA 600/3-78-105, 455 p. 